\title{
Comprehensively Improved Electrochemical Performance of Lithium-Sulfur Batteries by "Chemical Anchors" and Lithium Anode Modification
}

\author{
Zhipeng Zeng \\ West Virginia University, zz0014@mix.wvu.edu
}

Follow this and additional works at: https://researchrepository.wvu.edu/etd

Part of the Materials Science and Engineering Commons

\footnotetext{
Recommended Citation

Zeng, Zhipeng, "Comprehensively Improved Electrochemical Performance of Lithium-Sulfur Batteries by "Chemical Anchors" and Lithium Anode Modification" (2020). Graduate Theses, Dissertations, and Problem Reports. 7758.

https://researchrepository.wvu.edu/etd/7758

This Dissertation is protected by copyright and/or related rights. It has been brought to you by the The Research Repository @ WVU with permission from the rights-holder(s). You are free to use this Dissertation in any way that is permitted by the copyright and related rights legislation that applies to your use. For other uses you must obtain permission from the rights-holder(s) directly, unless additional rights are indicated by a Creative Commons license in the record and/ or on the work itself. This Dissertation has been accepted for inclusion in WVU Graduate Theses, Dissertations, and Problem Reports collection by an authorized administrator of The Research Repository @ WVU. For more information, please contact researchrepository@mail.wvu.edu.
} 


\title{
Comprehensively Improved Electrochemical Performance of Lithium-Sulfur Batteries by "Chemical Anchors" and Lithium Anode Modification
}

\author{
Zhipeng Zeng \\ Dissertation submitted to the \\ Benjamin M. Statler College of Engineering and Mineral Resources \\ at West Virginia University \\ in partial fulfillment of the requirements for the degree of \\ Doctor of Philosophy in \\ Material Science and Engineering \\ Xingbo Liu, Ph.D., Chair \\ Harry Finklea, Ph.D. \\ Kostas Sierros, Ph.D. \\ Terence Musho, Ph.D. \\ Wenyuan Li, Ph.D. \\ Department of Mechanical and Aerospace Engineering
}

Morgantown, West Virginia

2020

Keywords: lithium-sulfur batteries, chemical anchor, polysulfides, electrocatalysis, lithium anode protection, electrochemical performance

Copyright 2020 Zhipeng Zeng 


\section{Abstract \\ Comprehensively Improved Electrochemical Performance of Lithium-Sulfur Batteries by "Chemical Anchors" and Lithium Anode Modification}

\section{Zhipeng Zeng}

Lithium-sulfur battery is considered to be one of the most promising contenders for the next generation high-energy storages due to their high theoretical energy density $\left(\sim 2600 \mathrm{~W} \mathrm{~h} \mathrm{~kg}^{-1}\right)$. However, a series of issues, especially for the dissolution of lithium polysulfides (LiPSs) with their "shuttle effect" and dendrite formation on the lithium anode, greatly limit their widely commercial applications. Starting from a brief overview of conventional methods to solve these problems, the achievements spotlighted in this research work mainly focus on the structure design of cathode materials by employing "chemical anchors" to effectively suppress the diffusion of LiPSs, as well as the Li anode modification to suppress the dendrite formation, thus the electrochemical performance of Li-S batteries can be comprehensively improved. The synthetic methods, characterization techniques with electrochemical performance are presented. Further work plans and implications are proposed regarding optimize the structure of materials, preparation technology, and to better understand the role of these "chemical anchors" and the protection mechanism of Li anode. Proposing with some perspectives and future research efforts, this research is hoped to provide an in-depth understanding and offer avenues in the rational design of Li-S batteries with long cycle life and high energy/power density in the near future. 


\section{Acknowledgements}

First of all, I would like to deeply thank my supervisor, Prof. Xingbo Liu, for his financial support, patient encouragement, inspiration, and guidance during my Ph.D. study. I particularly appreciate that he always allowed me to try whatever topics I was interested in and always encouraged me to enjoy the research.

Second, I would express my gratitude to my committee members: Dr. Harry Finklea, Dr. Kostas Sierros, Dr. Terence Musho, and Dr. Wenyuan Li, both for the time and constructive advice on my research. I would also like to thank Dr. Marcela Redigolo and Dr. Qiang Wang in the WVU Shared Research Facilities group, who offer invaluable guidance in my sample characterizations and data analysis.

Third, I would like to thank all the colleagues in my research group, from whom I received lots of help and support. A special thank goes to Dr. Wei Li, who always provides useful suggestions for my experimental design and paper writing. I'm also indebted as much to Dr. Greg Collins, who helps me build the experimental set-up and repair the equipment. And Dr. Tao Yang, who helps me work on the modeling section of my research. My gratitude also goes to other current colleagues, including Dr. Shanshan Hu, Dr. He Qi, Nan Zhang, and Hanchen Tian, et al., and previous members of the group: Dr. Xinxin Zhang and Dr. Meng Yao.

At last, I would like to thank my parents, brother, and all my family and friends, for their unconditional love and support. Without them, I cannot finish my Ph.D. study at West Virginia University. 


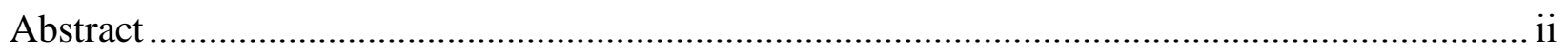

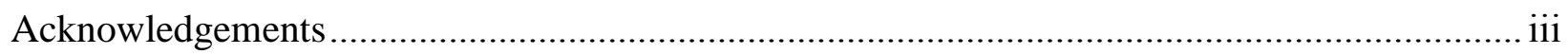

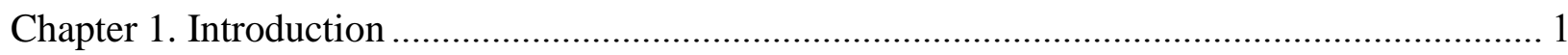

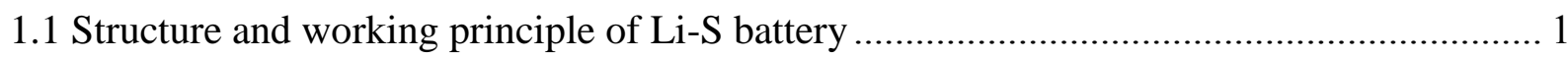

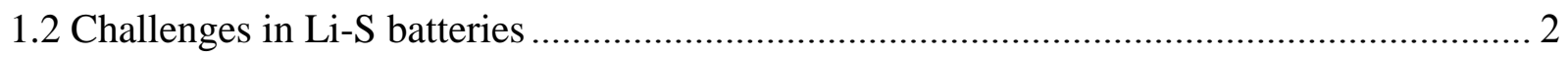

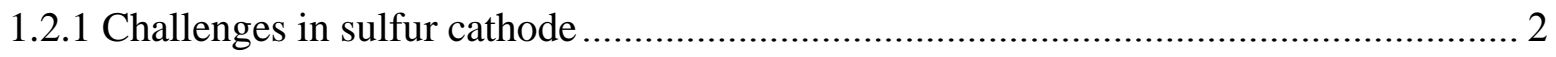

1.2.2 Challenges in lithium metal anode....................................................................... 4

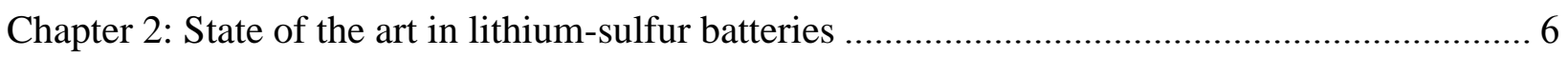

2.1 Conventional sulfur electrodes with physical absorption of LiPSs .................................... 6

2.2. Sulfur immobilization by "chemical anchors".............................................................. 12

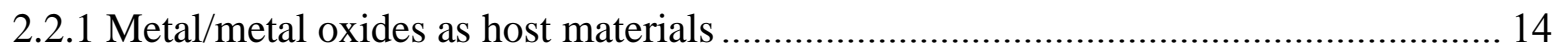

2.2.2 Metal sulfides as the host or additive materials..................................................... 16

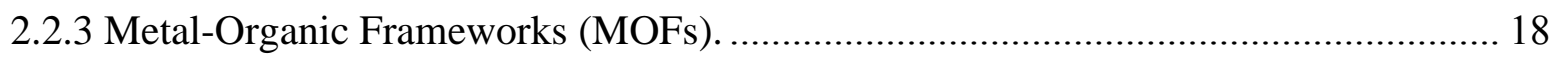

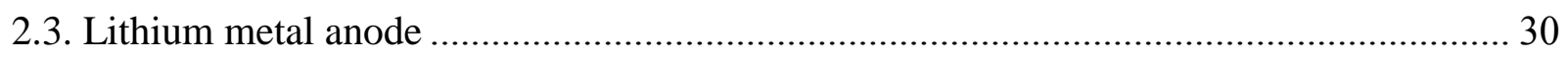

2.3.1 Mechanism and modeling of Li dendrite growth................................................. 30

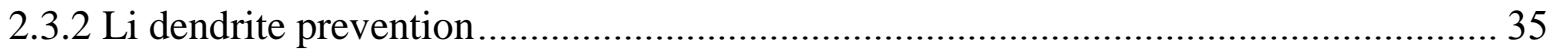

Chapter 3: Research objectives and approach ......................................................................... 43

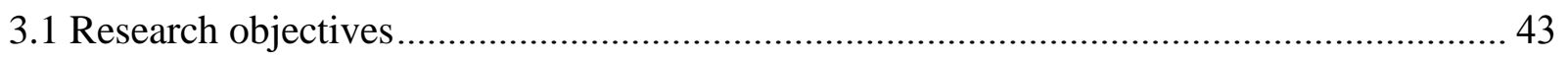

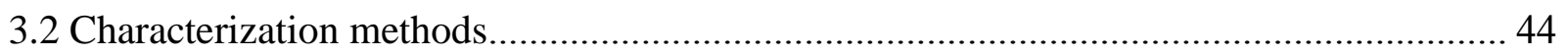

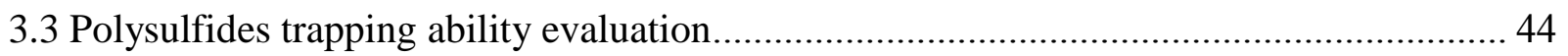


3.4 Electrochemical measurements

Chapter 4: Nanosized $\mathrm{FeS}_{2}$ Particles Caged in the Hollow Carbon Shell as a Robust Polysulfide

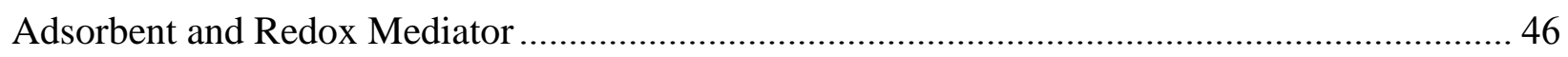

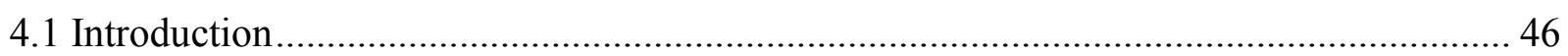

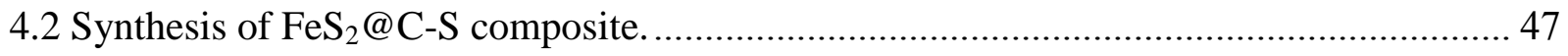

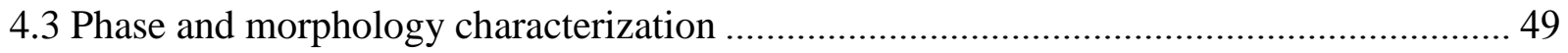

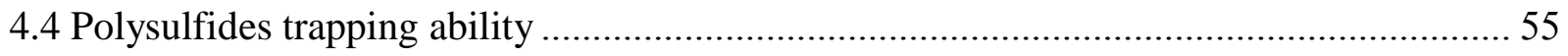

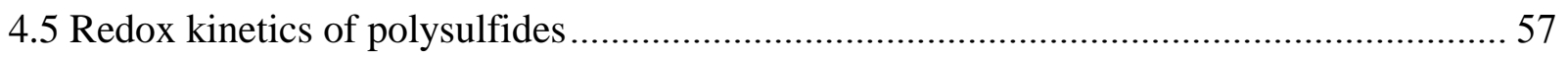

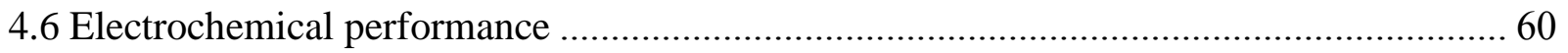

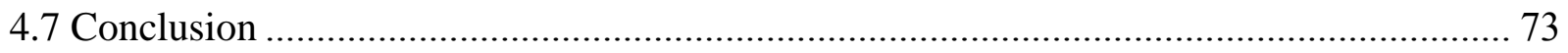

Chapter 5: Programmed Design of a Li-S Battery Cathode by Integrating Functional Units ...... 74

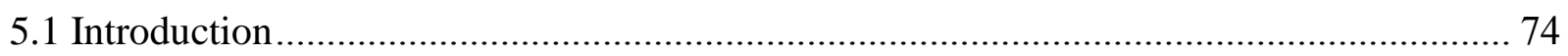

5.2 Synthesis of S/CNF-HC-Ni $\mathrm{S}_{2}$ electrode and related control samples. ......................... 76

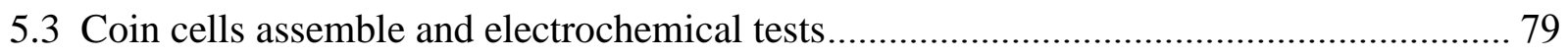

5.4 Physical and chemical structure characterizations ............................................... 80

5.5 Electrochemical performance with low and high sulfur loading ................................ 86

5.6 Analysis of redox kinetics and polysulfide trapping ability ..................................... 92

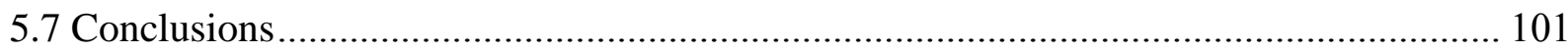

Chapter 6: Li metal anode modification ....................................................................... 102

6.1 Stable Li metal anode enabled by ionic conducting high entropy oxide protective layer 102 


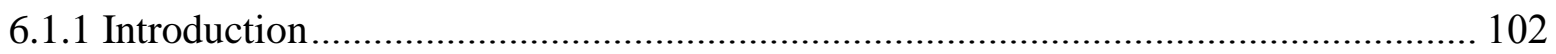

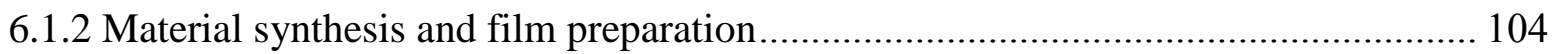

6.1.3 Morphology and structure characterization .......................................................... 105

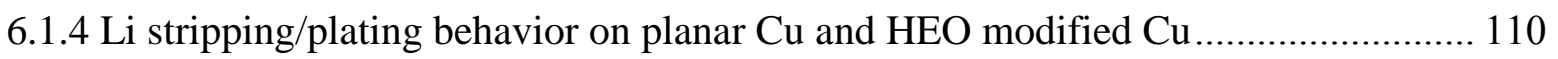

6.1.5 Electrochemical performance of symmetric cells................................................. 114

6.2 3D Conductive Hairy Foam as Stable Lithium Deposition Medium................................ 117

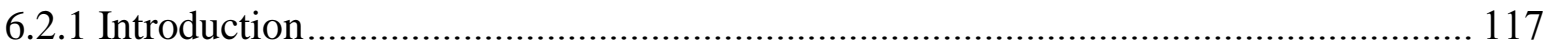

6.2.2 Preparation of CNF@ Ni hairy foam and material characterization ........................... 119

6.2.3 Li stripping/plating behavior on planar $\mathrm{Cu}, 3 \mathrm{D} \mathrm{Ni}$ foam, and CNF@ Ni foam ......... 123

6.2.4 Electrochemical performance of symmetric cells ................................................. 133

6.2.5 Mechanism for stable Li stripping/plating electrochemistry ................................... 144

6.3 Electrochemical performance of Full cells ................................................................. 147

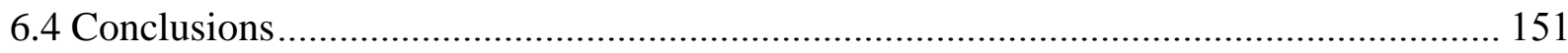

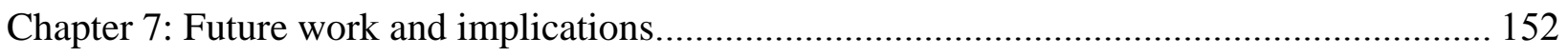

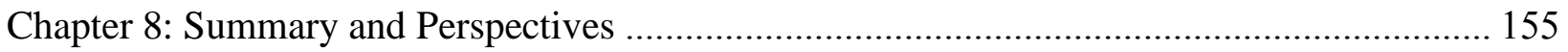

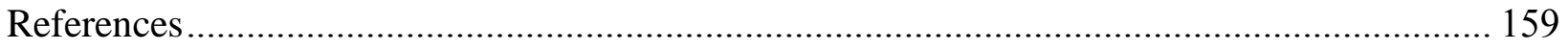




\section{List of Figures}

Figure 1 Structure and working principle of Li-ion battery (a) and Li-S battery (b) with the corresponding electrochemical reaction expression.

Figure 2 (a) The structure of sulfur allotropes by DFT calculation. (b) Typical charge /discharge curve for the sulfur cathode. (c) The mechanism of shuttle effect in Li-S batteries..................... 4

Figure 3 (a) Lithium metal anode fail mechanism. (b) Unstable SEI and the induced lithium dendrite formation 5

Figure 4 (a) Schematic illustrations of different hollow/porous carbonaceous materials as sulfur hosts. (b) Examples of commonly used conductive polymers in Li-S batteries...... 6 Figure 5 (a) The synthesis route for hollow CSC spheres. (b) Schematic of the process of the formation of HMMCNT-S materials, (c) FESEM, TEM, and STEM images of the HMMCNT-S composite and the corresponding elemental mapping. (d) Preparing S/(G-GCNs) composite with

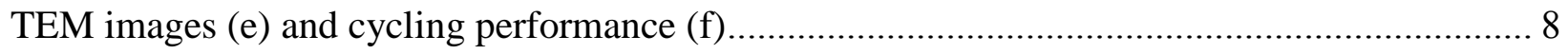
Figure 6 (a) Two-step synthesis route for S-PANI composite and (b) the schematic illustration of the structure effect of S-PANI on the long-term cycling performance. (c) The structure and functions of the PEMs and functionalized GS coating film with corresponding high-resolution TEM images of the HCSs/S-LBL (d) and HCSs/S composites after soaking in the $\mathrm{Li}_{2} \mathrm{~S}_{4}$ solutions

(e) and prolonged cycle performance at $1 \mathrm{~A} \mathrm{~g}^{-1}$ (f). 10

Figure 7 (a) the preparation process for MC-Cu-S composite, (b) XRD patterns of MC, Mixed MC-S, MC-S, MC-Cu, and MC-Cu-S. c) The proposed interaction mechanisms of polysulfide and $\gamma-\mathrm{MnO}_{2}$ on the surface. 15 
Figure 8 (a) Brief illustration of the fabrication of $\mathrm{FeS}_{2} @ \mathrm{~N}$-graphene (RT: room temperature), (b, c) TEM image, and HR-TEM image of $\mathrm{FeS}_{2} @ \mathrm{~N}$-graphene particles, (d, e) schematic diagrams for the reaction mechanism. 18 Figure 9 (a) Schematic illustration for the preparation of the $\mathrm{Cu}$-anchored cross-linked polymercoated sulfur electrode, (b) FT-IR spectra of the chemically anchored polymer-coated (CAPC) sulfur, (c) Raman spectra of the pristine sulfur and the $\mathrm{Cu}$-vinyl pyridine-anchored sulfur....... 20 Figure 10 (a) Schematic illustration of different MOF encapsulated sulfur, (b) TEM and SEM images of S@ZIF-8, S@ZIF-67, and S@HKUST-1 hybrids with corresponding elemental mapping as marked. (c) Synthesis of $\mathrm{S}_{8}$ loaded MOFs/CNT composite thin films, d) rate performances of S@HKUST-1/CNT electrode and e) the cycling performances of S@HKUST1/CNT, S@MOF-5/CNT, and S@ZIF-8/CNT electrodes, respectively..... 22 Figure 11 (a) SEM image of the Li surface of the Li cell cycled to the end of its life. (b) Schematic diagrams showing the separation of needle-like Li. (c) A description of the morphology and failure mechanisms of lithium electrodes and relevant AFM images. 31

Figure 12 For a given distance L between the electrodes, (a) if $J<J^{*}$, the system evolves to a steady state where the concentration varies linearly from $\mathrm{C}_{0}-\Delta \mathrm{C}$ at the negative electrode to $\mathrm{C}_{0}+\Delta \mathrm{C}$ at the positive electrode, (b) if $J<J^{*}$ (semi-infinite approximation), the ionic concentration drops to zero and the cell potential diverges at the Sand's time. (c) Schematic diagram of the galvanostatic polarization cell. (d) Concentration and (e) potential profiles in the galvanostatic cell. (f) Diagram of the region near the dendrite tip. (g) Typical growth profile for a lithium dendrite. 35

Figure 13 (a) Scheme of the procedures to prepare a 3D porous $\mathrm{Cu}$ foil from a planar $\mathrm{Cu}$ foil. Structural and morphological characterization results of the CMN. (b) Schematic illustration 
showing the preparation process of the CMN. (c) Low-magnification SEM image of the CMN. (d) Cross-sectional SEM image of spherical C and (e) sliced TEM image of a spherical C, which shows the onion-like structure. (f) Cross-sectional SEM image of the CMN. 38 Figure 14 (a) Fabrication process for the hollow carbon nanosphere-modified Cu electrode. SEM images of the carbon-coated polystyrene nanoparticle array at low (b) and high (c) magnifications. (d) Digital camera image of the as-fabricated hollow carbon nanosphere thin film after removal of the polystyrene template. (e) Cross-sectional SEM image of the hollow carbon nanospheres. (f) TEM image of the hollow carbon nanospheres, with a wall thickness of $\sim 20 \mathrm{~nm}$. (g) SEM image of the hollow carbon nanosphere thin-film peeled off the $\mathrm{Cu}$ substrate. (h) Schematic of the PEO-garnet hybrid ion-conducting membrane coated on Li metal. 40 Figure 15 (a) Schematic of the large capacity Li plating-stripping process for the Li$\mathrm{Sn} /$ garnet/Li-Sn symmetric cell. Cross-sectional SEM images of the Li-Sn alloy coated garnet before (b) and (c) after Li plating. (d) EDS mapping of the Li-plated area marked with a green dashed line in (c). Cross-sectional SEM images of the Li-Sn alloy coated garnet (e) before and (f) after Li stripping. (g) EDS mapping of the Li stripped area marked with a green dashed line in (f), where the leftover Sn does not show local accumulation at the interface. (h) Al-Li glassy phase enabled the intimate contact interface and uniform $\mathrm{Li}^{+}$flux. 42 Figure 16 Research approaches based on the sulfur cathode and lithium metal anode to realize the objectives of high-performance Li-S batteries....................................................... 43 Figure 17 Design thought for sulfur electrode development. 47 Figure 18 (a) formation mechanism of $\mathrm{Fe}_{2} \mathrm{O}_{3}$ nanotube and CVD apparatus. (b) The schemed synthesis process of $\mathrm{FeS}_{2} @ \mathrm{C}-\mathrm{S}$ composite with the mechanism of high $\mathrm{Li}^{+} / \mathrm{e}^{-}$flux and redox conversion kinetics. 48 
Figure 19 (a) XRD pattern of $\mathrm{FeS}_{2} @ \mathrm{C}-\mathrm{S}$ composite and standard XRD card of $\mathrm{FeS}_{2}$ and S. (b) XRD patterns of the pristine $\mathrm{Fe}_{2} \mathrm{O}_{3}$ nanotubes obtained from the hydrothermal process, $\mathrm{Fe}_{3} \mathrm{O}_{4} @ \mathrm{C}$, and $\mathrm{FeS}_{2} @ \mathrm{C}$ with corresponding standard PDF cards. TG curve of $\mathrm{FeS}_{2} @ \mathrm{C}$ (c) and FeS $@ \mathrm{C}-\mathrm{S}$ (d) composite recorded under air, $\mathrm{N}_{2}$ atmosphere, respectively. 50

Figure 20 SEM images of low magnification (a) and high magnification (b) of prepared $\mathrm{FeS}_{2} @ \mathrm{C}-\mathrm{S}$ composite. TEM images of $\mathrm{FeS}_{2} @ \mathrm{C}(\mathrm{c})$ and $\mathrm{FeS}_{2} @ \mathrm{C}-\mathrm{S}$ (e) with corresponding EDX results (d, f), respectively. (g) HR-TEM micrographs of the $\mathrm{FeS}_{2} @ \mathrm{C}-\mathrm{S}$ composite. (h) $\mathrm{N}_{2}$ adsorption/desorption isotherms and (i) pore size distribution of the corresponding samples..... 53 Figure 21 SEM images of pristine $\mathrm{Fe}_{2} \mathrm{O}_{3}$ nanotubes at low (a) and high magnification (b),

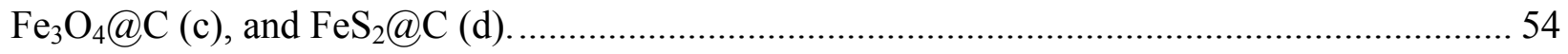
Figure 22 SEM images of $\mathrm{FeS}_{2} @ \mathrm{C}-\mathrm{S}$ and corresponding elemental maps of C (b), Fe (c), and S (d) and its EDX spectrum. 54

Figure 23 (a) Adsorption ability test of $\mathrm{MC}$ and $\mathrm{FeS}_{2} @ \mathrm{C}-\mathrm{S}$ electrodes with the representative lithium polysulfides (1 $\mathrm{M} \mathrm{Li}_{2} \mathrm{~S}_{6}$ ). (b) FTIR and (c) Raman curves for various samples, and highresolution XPS spectra of (d) C $1 s$, (e) Fe $2 p$, (f) $\mathrm{S} 2 p$, and (g) O $1 s$ peaks of fresh $\mathrm{FeS}_{2} @ \mathrm{C}-\mathrm{S}$ electrode film. 57

Figure 24 Typical CV curves of $\mathrm{FeS}_{2} @ \mathrm{C}-\mathrm{S}$ (a) and MC/S (b) cathodes. Comparison of CV peak voltages (c), onset potentials (d), cathodic (e), and anodic (f) polarization curves and Tafel plots (g) of asymmetrical Li-S cells consisting of both $\mathrm{FeS}_{2} @ \mathrm{C}-\mathrm{S}$ or MC/S cathode and Li anode. (h) $\mathrm{CV}$ and (g) EIS Nyquist curves of symmetric cells of $\mathrm{FeS}_{2} @ \mathrm{C}$ or $\mathrm{MC}$ host electrodes. 59 Figure 25 Equivalent circuit used for fitting the EIS spectra of symmetrical cells in Figure 5i and the corresponding fitting results 60 
Figure 26 (a) Cycle performance of $\mathrm{FeS}_{2} @ \mathrm{C}-\mathrm{S}$ electrode at $0.2 \mathrm{~A} \mathrm{~g}^{-1}$, (b) rate capability at various C-rates and the corresponding charge/discharge curves (c), and (d) long-term cycle performance of $\mathrm{FeS}_{2} @ \mathrm{C}-\mathrm{S}$ electrode at the current density of $5 \mathrm{~A} \mathrm{~g}^{-1}$

Figure 27 (a) cycle performance of MC/S at $0.2 \mathrm{C}$. Charge/discharge curves of MC/S (b) and $\mathrm{FeS}_{2} @ \mathrm{C}-\mathrm{S}$ (c) electrodes. (d) Rate capability from $0.5 \mathrm{C}$ to $5 \mathrm{C}$ with corresponding charge/discharge curves of MC/S electrode (e). 63

Figure 28 (a) Cyclic voltammograms of $\mathrm{FeS}_{2} @ \mathrm{C}-\mathrm{S}$ electrode at varying scan rates from 0.2 to $1.0 \mathrm{mV} \mathrm{s}^{-1}$ and (b) peak currents as a function of the square root of the scan rate. 65 Figure 29 Cycle performance of $\mathrm{FeS}_{2} @ \mathrm{C}-\mathrm{S}$ electrodes at the current densities of $1 \mathrm{C}$ (a), 2 C (b) for 100 cycles. (c) Cycle performance of $\mathrm{FeS}_{2} @ \mathrm{C}-\mathrm{S}$ electrode with $\mathrm{S}$ loading of $3.15 \mathrm{mg} \mathrm{cm}^{-2}$ at the current densities of $0.2 \mathrm{C}$ for 60 cycles........ 66 Figure 30 SEM images of fresh (a) and cycled (b) MC/S electrode film with corresponding EIS Nyquist curves at different cycles (c). SEM images of fresh (d) and cycled (e) $\mathrm{FeS}_{2} @ \mathrm{C}$-S electrode film with corresponding EIS Nyquist curves at different cycles (f), and high-resolution XPS spectra of (g) C $1 s$, (h) S $2 p$, (i) Li 1s peaks of cycled FeS 2 @C-S electrode film. 71

Figure 31 (a) SEM images of cycled MC/S electrode film and corresponding elemental maps of C (b), S (c), O (d). 72

Figure 32 (a) SEM images of cycled $\mathrm{FeS}_{2} @ \mathrm{C}-\mathrm{S}$ electrode film and corresponding elemental maps of C (b), Fe (c), S (d), and O (e). 72

Figure 33 Design route for high sulfur loading electrode development by integrating 3D conductive Ni foam network, dual functional carbon layer with large surface area. 74 Figure 34 Schematic illustration of (a) the flagellate-like S/CNF-HC-Ni $\mathrm{S}_{2}$ cathode and giardia lamblia. (b) The mechanism of cooperative lithiophilic and sulfiphilic interfaces of S-doped 
carbon and $\mathrm{Ni}_{3} \mathrm{~S}_{2}$ for enhanced adsorption and electrocatalytic conversion of lithium polysulfides.

Figure 35 (a) XRD pattern of the $\mathrm{NiFe}_{2} \mathrm{O}_{4}$-modified $\mathrm{Ni}$ foam after the hydrothermal process and standard XRD cards of $\mathrm{NiFe}_{2} \mathrm{O}_{4}$ and Ni. (b) Photographs of Ni foam discs before (left) and after (right) the hydrothermal surface modification treatment. SEM images (c, d), EDX spectrum (e), and quantitative analysis (f) of $\mathrm{NiFe}_{2} \mathrm{O}_{4}$-modified $\mathrm{Ni}$ foam. 77 Figure 36 Schematic illustrations of the whole programmed fabrication process for synthesizing $\mathrm{S} / \mathrm{CNF}-\mathrm{HC}-\mathrm{Ni}_{3} \mathrm{~S}_{2}$. 79 Figure 37 (a) The magnified XRD pattern of S/CNF-HC-Ni $\mathrm{S}_{2}$ and standard XRD cards of $\mathrm{Ni}_{3} \mathrm{~S}_{2}$ and S. SEM images of the top view of (b) S/CNF-HC-Ni $\mathrm{S}_{2}$ electrode. Cross-sectional SEM images of (c) Ni foam after the CVD process and (d) S/CNF-HC-Ni $\mathrm{S}_{2}$ electrode. Inset of (c) is a lateral view of the giardia lamblia structure. (e) EDX line scan profile and (f) EDX spectrum of $\mathrm{S} / \mathrm{CNF}-\mathrm{HC}-\mathrm{Ni}_{3} \mathrm{~S}_{2}$ with the corresponding elemental quantification in the inset. 82 Figure 38 SEM images of fresh Ni foam at low (a) and high (c) magnification, and the SEM images for hydrothermally treated $\mathrm{Ni}$ foam after CVD process $(\mathrm{b}, \mathrm{d})$ with the flourishing growth of carbon nanofibers (CNFs) on the surface. 83 Figure $39 \mathrm{SEM}$ images of pristine $\mathrm{S} / \mathrm{CNF}-\mathrm{HC}-\mathrm{Ni}_{3} \mathrm{~S}_{2}$ and corresponding elemental maps of C (b), $\mathrm{Ni}(\mathrm{c})$, and S (d) and its EDX spectrum. 83 Figure 40 FTIR (a) and Raman spectra (b), and high-resolution XPS spectra of C $1 s$ (c), S $2 p$ (d), $\mathrm{Ni} 2 p(\mathrm{e})$ and $\mathrm{O} 1 s(\mathrm{f})$ peaks of pristine $\mathrm{S} / \mathrm{CNF}-\mathrm{HC}-\mathrm{Ni}_{3} \mathrm{~S}_{2}$ electrode. 86 Figure $41 \mathrm{CV}$ curves of S/CNF-HC-Ni $\mathrm{S}_{2}$ (a) and S/CNF-HC (b) cathodes for 5 cycles, the cycle performance of S/CNF-HC-Ni $\mathrm{S}_{2}$ and $\mathrm{S} / \mathrm{CNF}-\mathrm{HC}$ cathodes at $0.2 \mathrm{C}$ (c). The charge/discharge curves of $\mathrm{S} / \mathrm{CNF}-\mathrm{HC}-\mathrm{Ni}_{3} \mathrm{~S}_{2}$ and $\mathrm{S} / \mathrm{CNF}-\mathrm{HC}$ electrode $(\mathrm{d}, \mathrm{e})$. The rate capability (f) and 
discharge-charge profiles of the S/CNF-HC- $\mathrm{Ni}_{3} \mathrm{~S}_{2}$ electrode at various current densities $(\mathrm{g})$. Long-term cycles of S/CNF-HC-Ni $\mathrm{S}_{2}$ cathode at a current density of $5 \mathrm{C}$ (h) for Li-S batteries.

Figure 42 Cycle performance of $\mathrm{S} / \mathrm{CNF}-\mathrm{HC}-\mathrm{Ni}_{3} \mathrm{~S}_{2}$ electrodes on return to $0.2 \mathrm{C}$ for 100 cycles (a), and $2 \mathrm{C}$ for 300 cycles (b). Cycle performance of S/CNF-HC-Ni $\mathrm{S}_{2}$ electrode with a relatively high loading tested at $0.2 \mathrm{C}$ (a) and corresponding charge-discharge curves (b)........ 90 Figure 43 Comparison of CV peak voltages (a), onset potentials (b), cathodic (c) and anodic (d) polarization curves and Tafel plots (e) of asymmetrical Li-S cells consisting of both S/CNF-HC$\mathrm{Ni}_{3} \mathrm{~S}_{2}$ or S/CNF-HC cathode and $\mathrm{Li}$ anode. (f) $\mathrm{CV}$ curves and (g) EIS Nyquist curves of symmetric cells of CNF-HC-Ni $\mathrm{S}_{2}$ or CNF-HC electrodes. (h) C $1 s$ and (i) S $2 p$ XPS spectra of S/CNF-HC-Ni $\mathrm{S}_{2}$ cathode in asymmetrical Li-S cells after 100 cycles at discharged state......... 94 Figure 44 Photographs of the cycled Li-S cells of (a) S/CNF-HC and (b) S/CNF-HC-Ni $\mathrm{S}_{2}$ cathodes after 100 cycles and (c) S/CMP after 30 cycles with the corresponding separators, $\mathrm{Li}$ anodes and respective visualized cathodes soaked in dimethoxyethane solvent. 96 Figure 45 Charge/discharge profiles for the $\mathrm{Ni}_{3} \mathrm{~S}_{2} / \mathrm{Ni}$ electrode. The $\mathrm{Ni}_{3} \mathrm{~S}_{2} / \mathrm{Ni}$ electrodes were prepared by directly mixing the $\mathrm{Ni}$ foam discs and sulfur powder for the thermal sulfurization at $300{ }^{\circ} \mathrm{C}$ for $1 \mathrm{~h}$ in $\mathrm{Ar}$. The $\mathrm{Ni}_{3} \mathrm{~S}_{2} / \mathrm{Ni}$ electrode contributes to limited capacity within the potential window of 1.7-3.0 V for testing $\mathrm{Li}-\mathrm{S}$ cells. 99

Figure 46 SEM images of CMPs coated Ni foam at low (a) and high (b) magnification. SEM images of S/CMP electrode at low (c) and high (d) magnification. (e) XRD pattern of pristine S/CMP electrode and the corresponding cycle performance at $0.2 \mathrm{C}$ (f). 100 Figure 47 SEM images of S/CNF-HC-Ni $\mathrm{S}_{2}(\mathrm{a}-\mathrm{b})$ and S/CMP (c-d) electrodes after 100 cycles. 
Figure 48 SEM image (a), EDX spectrum (b) of S/CNF-HC-Ni $3_{3} \mathrm{~S}_{2}$ electrode and corresponding elemental maps with C (c), S (d), O (e), and Ni (f) after 100 cycles. 101

Figure 49 Schematic diagrams of the different Li anode structures. (a) Dendritic Li growth on pure lithium anode with uniform $\mathrm{Li}^{+}$flux and unstable SEI. (b) Modifying the Li foil with $\mathrm{Li}^{+}$ conducting high entropy oxide layer with uniform $\mathrm{Li}^{+}$flux and stable interface. 103 Figure 50 (a) XRD diffraction patterns of the series $(\mathrm{MgCoNiZnCu})_{1-\mathrm{x}} \mathrm{Li}_{\mathrm{x}} \mathrm{O}(\mathrm{x}=0$ to 0.5$)$, and the corresponding enlarged patterns at $2 \theta$ between $36^{\circ}$ and $44^{\circ}(\mathrm{b})$, and $61^{\circ}$ and $64^{\circ}(\mathrm{c})$. 105

Figure 51 Comparison of XPS spectra for different $\mathrm{HEO}_{\mathrm{x}}$ including $\mathrm{Li}_{0}, \mathrm{Li}_{0.33}$, and $\mathrm{Li}_{0.5}$. (a) $\mathrm{Mg}$ 1s, (b) Co 2p, (c) Ni 2p, (d) Cu 2p, (e) Zn 2p3, and (f) Li 1s spectra. 107

Figure 52 SEM images of synthesized $\mathrm{HEO}_{\mathrm{x}}$. (a) $\mathrm{x}=0$, (b) $\mathrm{x}=0.05$, (c) $\mathrm{x}=0.1$, (d) $\mathrm{x}=0.33$, (e, f) low and high magnification images of $\mathrm{Li}_{0.5}(\mathrm{x}=0.5)$. (g) The surface of $\mathrm{Li}_{0.5}$ particle and the corresponding EDX mapping (h). 108

Figure 53 (a) XRD profile of PEO-Li $\mathrm{Li}_{0.5}$ hybrid film on the Li foil. (b) Cross-sectional SEM image showing the thickness of the PEO- $\mathrm{Li}_{0.5}$ film on the Li foil. (c) Surface morphology of the PEO-Li ${ }_{0.5}$ film with the corresponding elemental EDX mapping (d-i). 110 Figure 54 The typical voltage profiles of the $\mathrm{Cu} \| \mathrm{Li}(\mathrm{a}, \mathrm{c}, \mathrm{e})$ and $\mathrm{Li}_{0.5}-\mathrm{Cu} \| \mathrm{Li}(\mathrm{b}, \mathrm{d}, \mathrm{f})$ half cells at current densities of $0.5(a, b), 1(c, d)$, and $2(e, f) \mathrm{mA} \mathrm{cm}^{-2}$. 112

Figure 55 Surface (a, d) and cross-sectional (b, e) SEM images of bare $\mathrm{Cu}$ foil $(\mathrm{a}, \mathrm{b})$ and $\mathrm{Li}_{0.5}-\mathrm{Cu}$ (d, e) after 50 cycles of stripping and plating with corresponding EIS Nyquist curves (c, f). The high magnification SEM images of corresponding Cu layer (g) and HEO layer (h), (i) Line-scan of the $\mathrm{HEO}$ coated $\mathrm{Cu}$ electrode after 50 cycles of Li stripping and plating. 114 
Figure 56 Long-term cycle stability of symmetrical cells for $\mathrm{Li} \| \mathrm{Li}, \mathrm{Li}_{0.33}|| \mathrm{Li}_{0.33}$, and $\mathrm{Li}_{0.5}|| \mathrm{Li}_{0.5}$ symmetric cells 0.5 (a), 1 (b), and 2 (c) $\mathrm{mA} \mathrm{cm}^{-2}$ with constant capacity of $1 \mathrm{mAh} \mathrm{cm}$ for 100 hours. 116

Figure 57 Galvanostatic cycling performance of $\mathrm{Li}_{0.5}|| \mathrm{Li}_{0.5}$ symmetric cell at a stripping/plating capacity of $5 \mathrm{mAh} \mathrm{cm}^{-2}$ at $1 \mathrm{~mA} \mathrm{~cm}$. 117

Figure 58 (a) Schemed synthesis process of CNF@Ni foam, (b) comparison of Li deposition behavior for different host electrodes: planar Cu, 3D Ni foam, and 3D CNF@Ni foam. 120 Figure 59 (a) XRD pattern of CNF@Ni and standard XRD card of Ni and graphite, Raman (b) and C 1s XPS (c) spectra of CNF@Ni. SEM images of CNF@Ni at low (d) and high (e) magnification with the corresponding cross-sectional image (f).

Figure 60 Surface SEM image of CNF@Ni and corresponding elemental maps of overlap (b), C (c) and $\mathrm{Ni}(\mathrm{d})$. 122

Figure 61 Cross-section SEM image of CNF@Ni and corresponding elemental maps of overlap (b), C (c) and $\mathrm{Ni}(\mathrm{d})$ 123

Figure 62 Morphological evolution of Li plating/stripping on planar $\mathrm{Cu}$, including pristine $\mathrm{Cu}(\mathrm{a})$, and after plating at 0.2 (b), 0.6 (c), $1 \mathrm{mAh} \mathrm{cm}^{-2}$ (d) of Li onto $\mathrm{Cu}$, and after stripping at 0.2 (e), 0.6 (f), $1 \mathrm{mAh} \mathrm{cm}^{-2}$ (g) of Li from Cu substrate. High magnification SEM images of d point (h) and g point (i). (j) Corresponding discharge/charge voltage profile of $\mathrm{Cu} \| \mathrm{Li}$ half-cell. 125 Figure 63 Morphological evolution of Li plating/stripping on pure Ni foam, including pristine $\mathrm{Ni}$ (a), and after plating at 0.2 (b), 0.6 (c), $1 \mathrm{mAh} \mathrm{cm}^{-2}$ (d) of Li onto the Ni foam, and after stripping at $0.2(\mathrm{e}), 0.6(\mathrm{f}), 1 \mathrm{mAh} \mathrm{cm}^{-2}$ (g) of Li from the Ni substrate. High magnification SEM images of d point (h) and g point (i). (j) Corresponding discharge/charge voltage profile of Ni\|Li half-cell. 
Figure 64 Morphological evolution of Li plating/stripping on the CNF@Ni, including pristine CNF@Ni (a), and after plating at 0.2 (b), 0.6 (c), 1 mAh cm ${ }^{-2}$ (d) of Li into the CNF@Ni, and after stripping at 0.2 (e), 0.6 (f), $1 \mathrm{mAh} \mathrm{cm}^{-2}$ (g) of Li from the CNF@Ni. (j) Corresponding discharge/charge voltage profile of CNF@Ni||Li half-cell.................................................... 128

Figure 65 EDX mapping of CNF@Ni foam electrode after Li plating for $1 \mathrm{mAh} \mathrm{cm}^{-2}$ (a-f) and corresponding EDX spectrum (g).

Figure $66 \mathrm{CE}$ of Li stripping from/plating on planar $\mathrm{Cu}, \mathrm{Ni}$ foam, and $\mathrm{CNF} @ \mathrm{Ni}$ foam at 0.5 (a), 1 (b), and $2 \mathrm{~mA} \mathrm{~cm}^{-2}$ with the constant areal capacity of $1 \mathrm{mAh} \mathrm{cm}^{-2}$ and the typical voltage profiles of the corresponding half cells (d-f). SEM images of $\mathrm{Cu}$ foil (g), Ni foam (h), and CNF@Ni foam (i) after 100 cycles stripping/plating at $1 \mathrm{~mA} \mathrm{~cm}^{-2}$ in half cells. 130 Figure 67 SEM images of planar Cu (a, d), Ni foam (b, e) and CNF@Ni foam (c, f) after 100 cycles at $0.5 \mathrm{~mA} \mathrm{~cm}{ }^{-2}$, and planar $\mathrm{Cu}(\mathrm{g}, \mathrm{j})$, Ni foam (h, k) and CNF@Ni foam (i, l) after 100 cycles at $2 \mathrm{~mA} \mathrm{~cm}^{-2}$ in the corresponding half cells: $\mathrm{Cu}\|\mathrm{Li}, \mathrm{Ni}\| \mathrm{Li}$, and CNF@Ni $\| \mathrm{Li}$. Figure 68 EIS Nyquist plots of half cells after 100 cycles at $0.5 \mathrm{~mA} \mathrm{~cm}{ }^{-2}$ (a), $1 \mathrm{~mA} \mathrm{~cm}^{-2}$ (b), and $2 \mathrm{~mA} \mathrm{~cm}^{-2}(\mathrm{c})$. 133

Figure 69 (a) Voltage-time profiles of Li stripping/plating with cycling capacity of $1 \mathrm{mAh} \mathrm{cm}^{-2}$ at $1 \mathrm{~mA} \mathrm{~cm}{ }^{-2}$ in $\mathrm{Cu}-\mathrm{Li}, \mathrm{Ni}-\mathrm{Li}$, and CNF@Ni-Li symmetric cells. Magnified voltage profiles during 0-10 h (b) and 100-110 h (c) periods.... 135

Figure 70 Symmetrical cells 0.5 and $2 \mathrm{~mA} \mathrm{~cm}^{-2}$ with a constant capacity of $1 \mathrm{mAh} \mathrm{cm}^{-2}$ for 100 hours for $\mathrm{Cu}-\mathrm{Li}$ and $\mathrm{Ni}-\mathrm{Li}$ symmetric cells...... 136 Figure 71 Electrochemical performances of CNF@Ni-Li symmetric cells. (a) Voltage profiles during Li plating/stripping processes under various current rates ranging from 0.5 to $5 \mathrm{~mA} \mathrm{~cm}^{-2}$. 
(b to c) Long-term cycling of CNF@Ni-Li symmetric cells with current densities of 0.5, 2, and 5 $\mathrm{mA} \mathrm{cm}{ }^{-2}$ with a deposition/stripping capacity of $1 \mathrm{mAh} \mathrm{cm}^{-2}$ 138

Figure 72 Magnified Li plating/stripping profiles of CNF@Ni-Li symmetric cells during different cycle periods. 139

Figure 73 Surface (a-c) and cross-sectional (d-f) SEM images of Cu-Li (a, d), Ni-Li (b, e), and CNF@Ni (c. f) electrodes after 100 cycles in the corresponding symmetric cells. (g) Summary of voltage hysteresis for the $\mathrm{Cu}-\mathrm{Li}$, Ni-Li, and CNF@Ni-Li symmetric cells at various current densities. (h) EIS Nyquist curves of symmetric cells after 100 cycles at various current densities of $0.5,1$, and $2 \mathrm{~mA} \mathrm{~cm}^{-2}$. 142 Figure 74 Illustration of the proposed electrochemical deposition processes of Li metal on planar $\mathrm{Cu}(\mathrm{a}), 3 \mathrm{D} \mathrm{Ni}$ foam (b), and 3D CNF@Ni foam (c), and models of the electric potential and current density distributions for a planar Cu electrode (d-f) and a CNF@Ni electrode (g-i).... 146 Figure 75 (a) cycle performance of $\mathrm{Li}_{0.5}|| \mathrm{LCO}$ and $\mathrm{Li} \| \mathrm{LCO}$ full cells with corresponding chargedischarge curves in (b, c), respectively. (d) Cycle performance of S/CNF-HC-Ni $\mathrm{S}_{2}$ cathodes using CNF@Ni-Li anode and pure Li metal anode. 


\section{List of Tables}

Table 1 Electrochemical performance of S/C composites in the previous literature................... 11

Table 2 Summary of electrochemical performance of the reported sulfur electrodes................ 24

Table 3 Summary of factors that may affect the electrochemical performance. ....................... 27

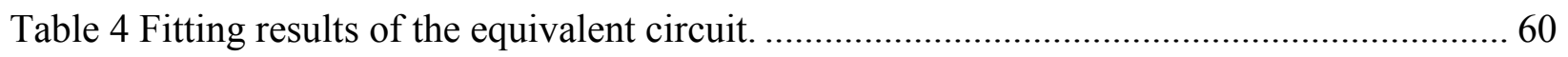

Table 5 The performance comparison of $\mathrm{FeS}_{2} @ \mathrm{C}-\mathrm{S}$ with reported cathodes. ........................... 67

Table 6 The performance comparison of this work with some other similar composites. ........... 91

Table 7 Ionic conductivity for $\mathrm{HEO}_{\mathrm{x}}$ samples .............................................................. 106

Table 8 The performance comparison of CNF@Ni-Li electrode with reported Li anodes........ 143

Table 9 Performance comparison of S/CNF-HC-Ni $\mathrm{S}_{2} \| \mathrm{CNF} @ \mathrm{Ni}-\mathrm{Li}$ with reported cathodes. . 150 


\section{Chapter 1. Introduction}

\subsection{Structure and working principle of Li-S battery}

Tremendous attention has been paid to the energy conversion and storage devices for their rapidly growing market and more requirement for nowadays' applications, ranging from portable electronics, electric tools to large renewable energy systems. ${ }^{[1]}$ Among them, Li-ion batteries are the most popular rechargeable batteries, since they can supply high specific energy and power densities with long cycle life, fast charging ability, and no memory effect. ${ }^{[2]}$ However, when expending to large-scale applications such as electric or hybrid electric vehicles and large-scale grids, Li-ion batteries are still far from the satisfaction in terms of limited energy density. The energy density for sulfur electrode five times higher than that of Li-ion batteries (2600 $\mathrm{Wh} \mathrm{kg}^{-1}$ vs. $500 \mathrm{Wh} \mathrm{kg}^{-1}$ ), makes it be a very promising candidate for large-scale emerging applications among the various candidates. ${ }^{[3]}$ Besides, the non-toxic sulfur is natural abundance, showing better environmental friendliness and a more attractive cost advantage in a commercial application. $^{[4]}$

The typical structures of Li-ion battery and Li-S battery are presented in Figure 1a and b. For commercial Li-ion batteries, the main components consist of a graphite anode, organic electrolyte with polymer separator, and layered $\mathrm{LiMO}_{2}(\mathrm{M}=$ transition metal elements such as $\mathrm{Co}$, $\mathrm{Ni}$, and $\mathrm{Mn}$ ). Li-ions intercalate into or deintercalate from the active materials reversible between the two electrodes with the total reaction:

$$
\mathrm{LiC}_{6}+2 \mathrm{Li}_{0.5} \mathrm{CoO}_{2} \leftrightarrow 6 \mathrm{C}+2 \mathrm{LiCoO}_{2}
$$

As to Li-S battery, the main components consist of a lithium metal anode and sulfur-carbon composite cathode with an organic liquid electrolyte in between. The total reaction is based on the conversion mechanism: 


$$
2 L i^{+}+2 e^{-}+S \leftrightarrow L i_{2} S
$$

Eqn. 2

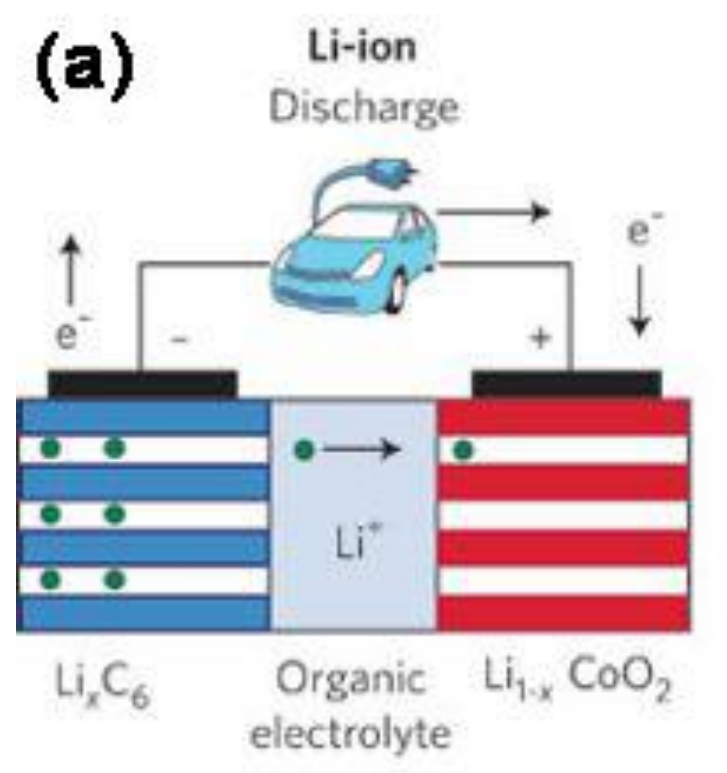

$3 / 2 \mathrm{C}_{6} \mathrm{Li}+\mathrm{Li}_{05} \mathrm{CoO}_{2} \leftrightarrow 3 \mathrm{C}+\mathrm{LiCoO}_{2}$
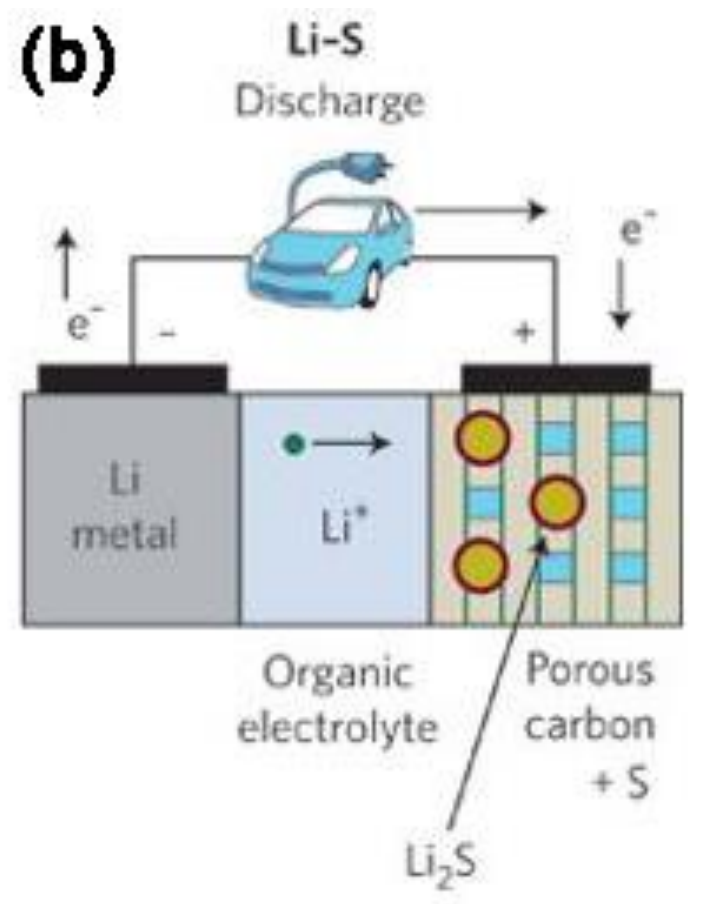

$$
2 \mathrm{Li}^{+}+2 \mathrm{e}^{-}+\mathrm{S} \leftrightarrow \mathrm{Li}_{2} \mathrm{~S}
$$

Figure 1 Structure and working principle of Li-ion battery (a) and Li-S battery (b) with the corresponding electrochemical reaction expression. ${ }^{[2]}$

\subsection{Challenges in Li-S batteries}

Despite the great advantages, the commercialized process for this promising material is still greatly hindered by a series of issues. According to the different components, the issues addressed in this research will be mainly focused on the sulfur cathode and Li metal anode.

\subsubsection{Challenges in sulfur cathode}

The first issue is the insulating of sulfur with an extremely low electronic conductivity of 5 $\times 10^{-30} \mathrm{~S} \mathrm{~cm}^{-1}$ at $25^{\circ} \mathrm{C}$ as well as the intermediate products, which significantly decreases the redox kinetics of conversion reactions and finally resulting in the low utilization of active 
material. The insulating nature of sulfur species also causes the sluggish rate capability with serious polarization especially when charge/discharge at large current rates.

Secondly, large volume change occurs during the charge/discharge process. Based on the conversion reaction mechanism, the sulfur particle will suffer from nearly $80 \%$ volume expansion, leading to the cracking and electrode pulverization of active particle, and fast capacity fading.

The most important issue is the dissolution of polysulfides and their "shuttle effect". The high solubility of long-chain polysulfides will be formed in commonly used liquid electrolyte systems when sulfur is gradually lithiated, resulting in the great loss of active material, poor cycle performance, and low Coulombic efficiency. Sulfur clusters show a range of structures with a strong tendency to catenation, which usually presents long homoatomic chains or homocyclic rings with various sizes, as shown in Figure 2a. ${ }^{[5]}$ Octasulfur (cyclo- $\left.\mathrm{S}_{8}\right)$ is the most common and stable allotrope at room temperature. ${ }^{[6]}$ According to the typical discharge behavior, cyclo- $\mathrm{S}_{8}$ is reduced with the ring-opening during the initial lithiation process, resulting in the formation of high-order lithium polysulfides $\operatorname{Li}_{2} S_{\mathrm{x}}(6 \leq \mathrm{x} \leq 8)$, corresponding to the upper plateau to $\sim 2.3 \mathrm{~V} .{ }^{[7]}$ With the further incorporation of lithium, short-chains $\operatorname{Li}_{2} \mathrm{~S}_{\mathrm{x}}(2<\mathrm{x} \leq 4)$ are formed yielding to a lower plateau at around $2.1 \mathrm{~V} \cdot{ }^{[8]}$ The final reaction product is $\mathrm{Li}_{2} \mathrm{~S}$ after a complete lithiation process, as shown in Figure $2 b .{ }^{[2]}$ The high-order polysulfides can be easily dissolved in the ether-based electrolytes, which then diffuse to the lithium anode side causing the side reacts with lithium, resulting in great loss of active material. The reduced products on lithium anode further diffuse back to the cathode resulting in infinite charge capacity, recycling corrosion on the lithium anode, and contributing to low Coulombic efficiency, which is known as the "shuttle effect", as illustrated in Figure 2c. ${ }^{[9]}$ 

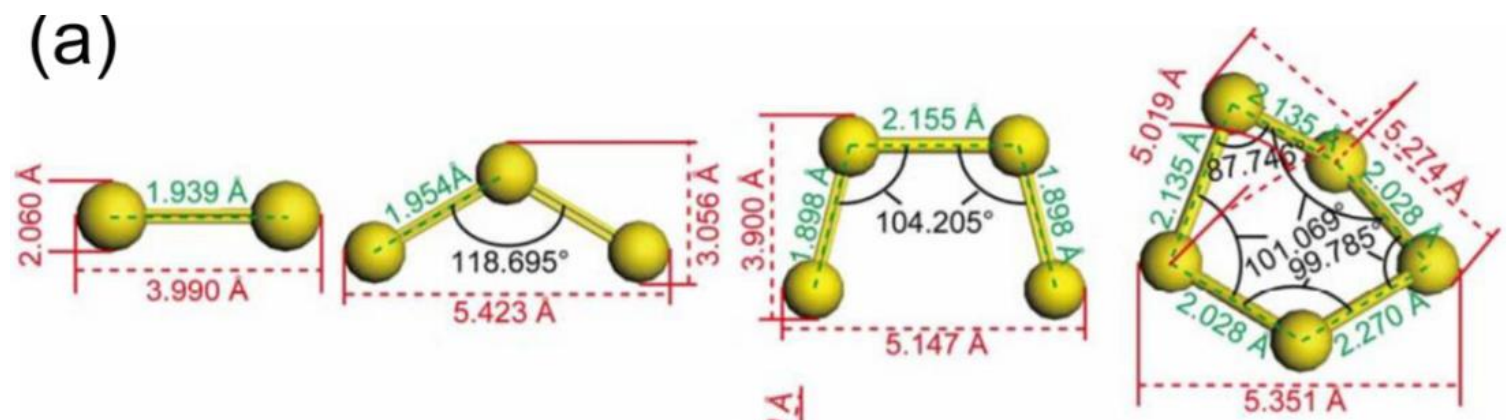

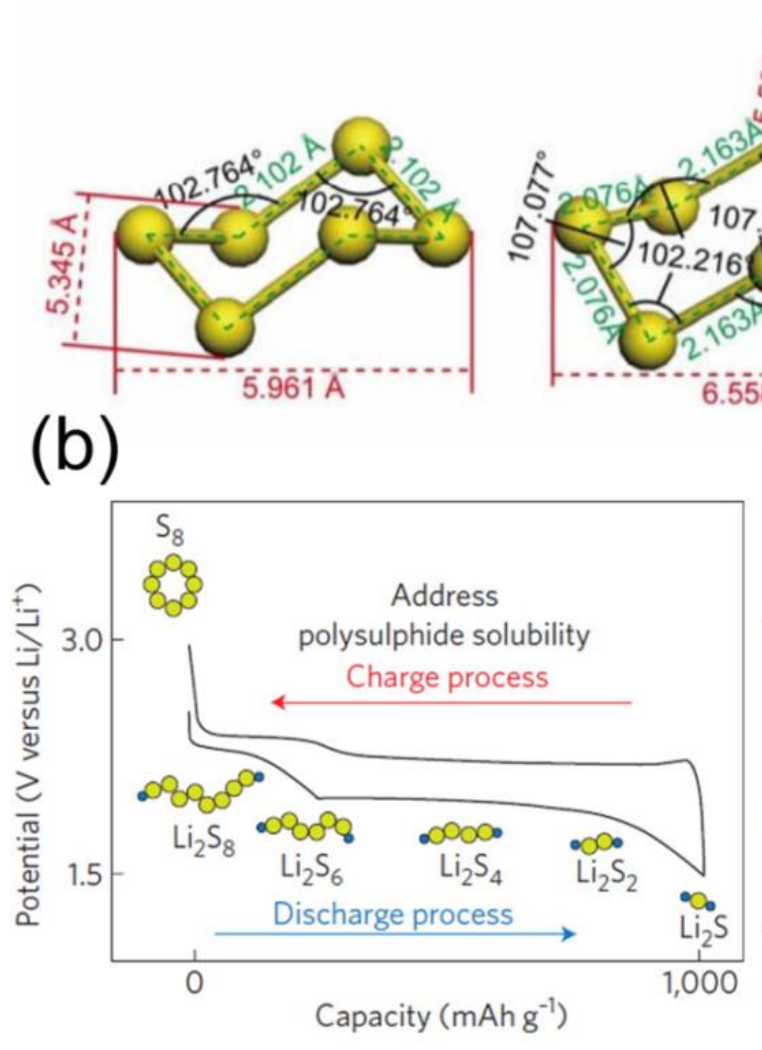

(c)
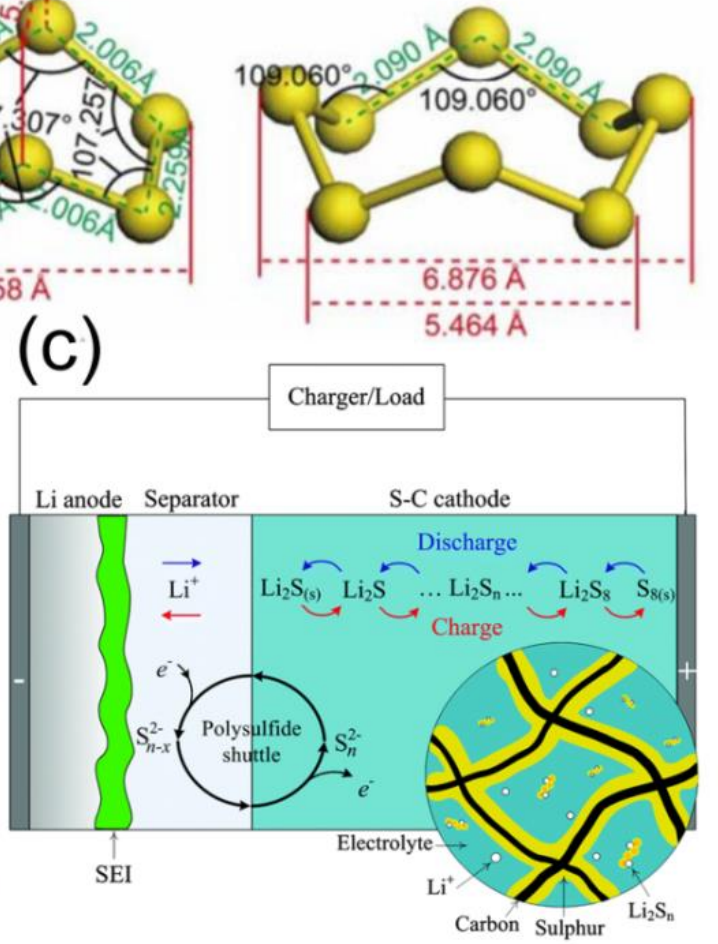

Figure 2 (a) The structure of sulfur allotropes by DFT calculation. ${ }^{[10]}$ (b) Typical charge /discharge curve for the sulfur cathode. ${ }^{[2]}$ (c) The mechanism of shuttle effect in Li-S batteries. ${ }^{[9]}$.

\subsubsection{Challenges in lithium metal anode}

Lithium metal is a key component of the Li metal rechargeable batteries due to its extremely high theoretical specific capacity $\left(3860 \mathrm{mAh} \mathrm{g}^{-1}\right)$, low density $\left(0.59 \mathrm{~g} \mathrm{~cm}^{-3}\right)$ and the lowest negative electrochemical potential $(-3.040 \mathrm{~V} v s$. the standard hydrogen electrode), thus can contribute to the high theoretical energy density. Unfortunately, the practical application of Li metal batteries has still been hindered by several critical challenges, as illustrated in Figure $3^{[11]}$ : 
(1) uncontrollable dendritic Li growth, which potentially penetrates the separator and causes an internal short circuit;

(2) the infinite volume change during cycling;

(3) the unstable interface between the reactive Li metal and the organic electrolyte, which leads to limited Coulombic efficiency during Li deposition/stripping;
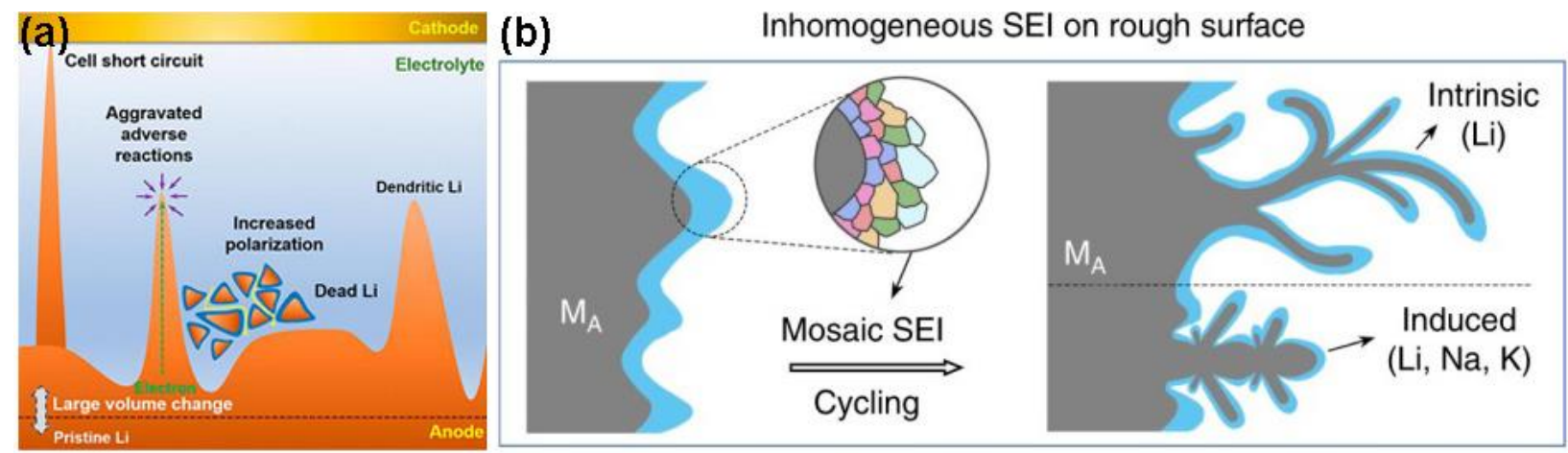

Figure 3 (a) Lithium metal anode fail mechanism. (b) Unstable SEI and the induced lithium dendrite formation. ${ }^{[11]}$

According to the challenges existing in the Li-S battery system, many methods have been conducted to address these issues both towards cathode and Li metal anode. Detailed discussions on various instruments/tools to modification of cathodes and protection of Li metal anodes will be provided in the following sections. 


\section{Chapter 2: State of the art in lithium-sulfur batteries}

\subsection{Conventional sulfur electrodes with physical absorption of LiPSs}

Coupling sulfur to carbonaceous materials (Figure 4a) or conductive polymer (Figure 4b) with fine structure has been widely regarded as the major approach to alleviating the drawbacks of the sulfur cathode. ${ }^{[12]}$ Commonly, these host materials can not only enhance electron transport during the electrode reaction but also absorb the polysulfides due to their large specific surface area. Lou's group recently reviewed various hollow carbon materials applied as sulfur host, as shown in Figure 4a. ${ }^{[13]}$ The most widely used method to impregnate sulfur in these complex shell structures is the so-called "Two-Step" heat treatment, which includes sulfur melting diffusion and sulfur vaporization. ${ }^{[12 a-d, 14]}$

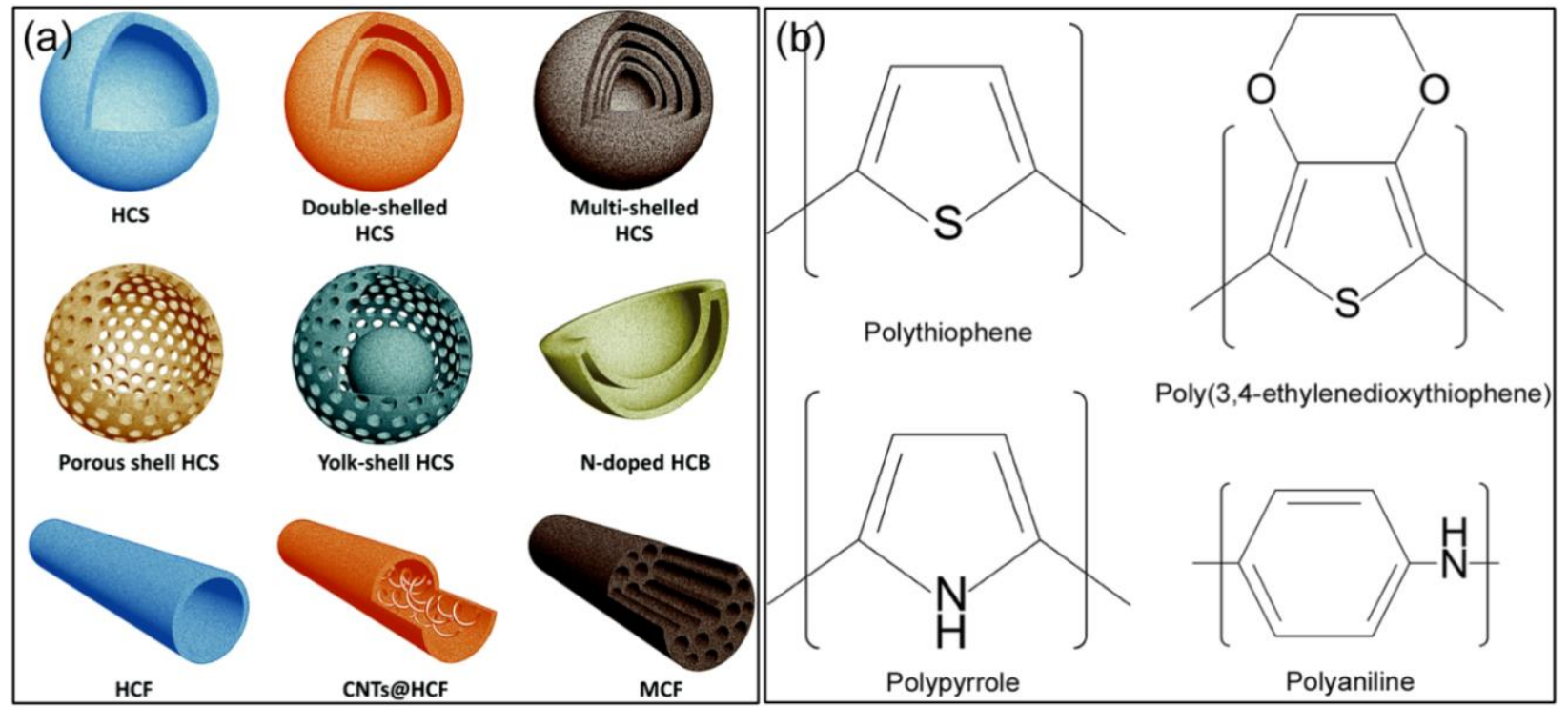

Figure 4 (a) Schematic illustrations of different hollow/porous carbonaceous materials as sulfur hosts. ${ }^{[13]}$ (b) Examples of commonly used conductive polymers in Li-S batteries. ${ }^{[15]}$

Sun and his co-workers synthesized hollow core-shell interlinked carbon spheres (CSC) through solution synthesis of polymer spheres followed by a pyrolysis process that occurred in 
the hermetical silica shell, as illustrated in Figure 5a. ${ }^{[16]}$ The CSC was further mixed with sulfur and heated at $155^{\circ} \mathrm{C}$ for $20 \mathrm{~h}$ to facilitate sulfur diffusion into the carbon host. A capacity of $c a$. $700 \mathrm{mAh} \mathrm{g} \mathrm{g}^{-1}$ could still be obtained at $6 \mathrm{C}$ for the as-prepared CSC-S electrode, indicating excellent cycle stability and rate capability. Mi et al. proposed a hierarchical macroporousmesoporous carbon nanotube (HMMCNT) as an efficient host (Figure 5b). ${ }^{[17]}$ The HMMCNT was obtained after removing Te particles by heat treatment and chemical etching. Sulfur was then infused into the micropores of HMMCNT through the impregnation method. The microporous outer layer and the inner hollow channel could be seen in the FESEM and TEM images (Figure 5c). The elemental mapping (Figure 5c) portrayed the homogeneous distribution of $\mathrm{S}$ in the micropores of HMMCNT. The novel structure of HMMCNTs was demonstrated to be an efficient host to ensure excellent electrochemical performance. In recent years, graphenebased composites have also been developed prosperously as promising candidates for electrode materials due to their excellent electrical conductivity, easily modified surface properties, and flexible mechanical characteristics. Graphenes, composited with carbon nanofibers, ${ }^{[18]}$ nanoparticles, ${ }^{[19]}$ nanotubes, ${ }^{[20]}$ have been frequently reported in the past few years. Recent literature published by Guo's group presented a graphitic carbon nanocage (GCN) for encapsulating sulfur (Figure 5d). ${ }^{[21]}$ The sandwich-type structure of the carbon layers embedded with abundant graphitic carbon nanocages, which uniformly distributed on the graphene backbone, could be further identified from the TEM images (Figure 5e). As expected, the S/(GGCNs) demonstrated a stable long-term cycle life with the high capacity of $706 \mathrm{mAh} \mathrm{g}^{-1}$ after 1000 cycles at $1 \mathrm{C}$ (Figure 5f). 

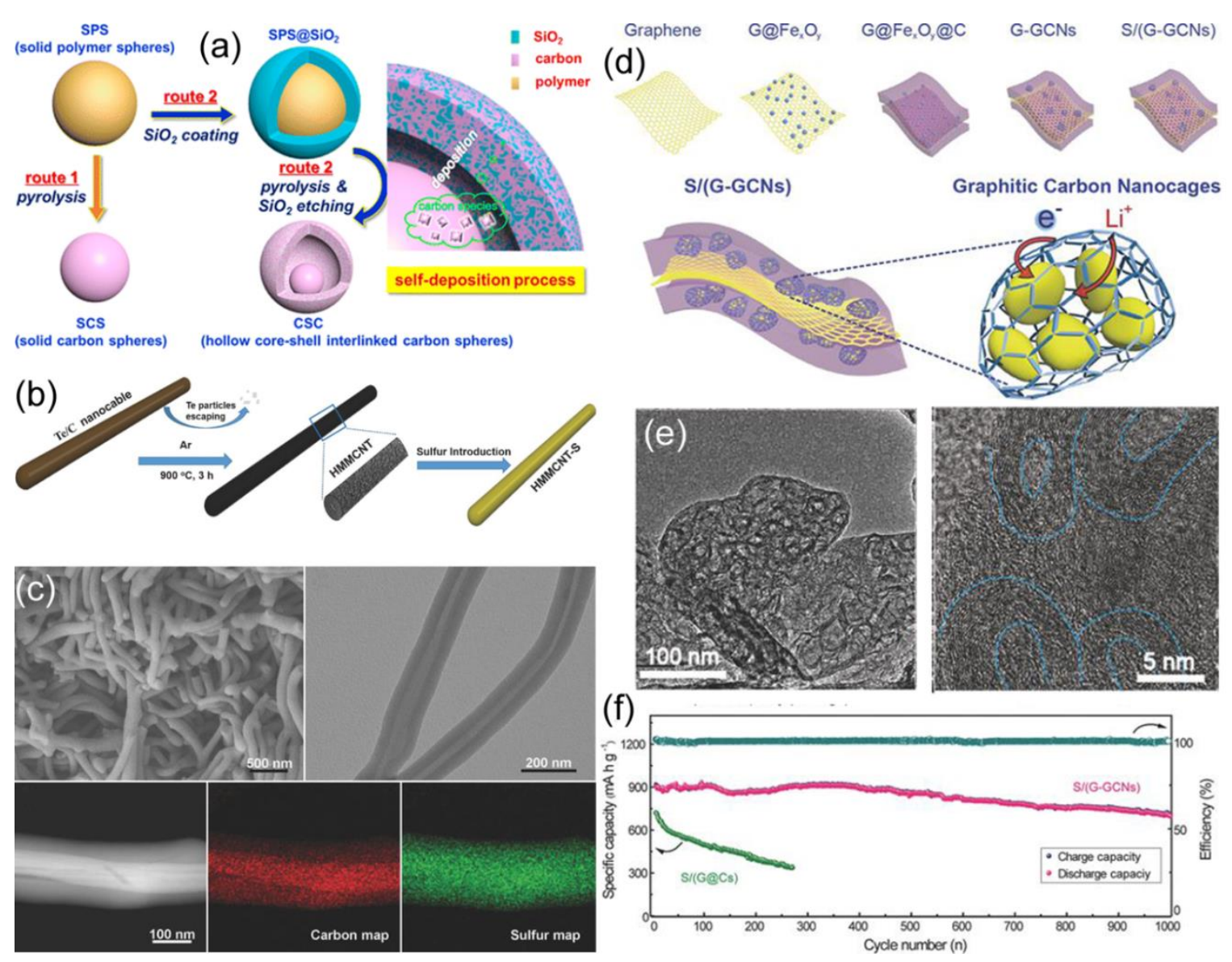

Figure 5 (a) The synthesis route for hollow CSC spheres. ${ }^{[16]}$ (b) Schematic of the process of the formation of HMMCNT-S materials, (c) FESEM, TEM, and STEM images of the HMMCNT-S composite and the corresponding elemental mapping. ${ }^{[17]}$ (d) Preparing S/(G-GCNs) composite with TEM images (e) and cycling performance (f). ${ }^{[21]}$

Extensive efforts have also targeted the synthesis of conductive polymers, for example, polyacetylene, polypyrrole, polythiophene, polyaniline, and their derivatives or composites. ${ }^{[22]}$ These conjugated polymers with unique mechanical properties, such as bendability, flexibility, or even stretchability, can not only act as a good sulfur host to accommodate the volume change but also, the synthetic tailorability and processability of conjugated polymers make them ideal candidates for electrochemical energy storage. ${ }^{[15]}$ The most popular conjugated polymers for energy storage are polythiophene (PT), poly (3,4-ethylenedioxythiophene) (PEDOT), 
polypyrrole (PPy), and polyaniline (PANi). The scheme of the corresponding chemical structure can be illustrated in Figure $4 \mathrm{~b} .{ }^{[15]} \mathrm{A}$ variety of approaches applied conductive polymers in lithium-sulfur batteries have been developed, such as sulfur particles coated with PPy, ${ }^{[12 \mathrm{e}]}$ coreshell-structured S-PPy, ${ }^{[23]}$ sulfur encapsulated in self-assembled PANI, ${ }^{[24]}$ core-shell-structured S-PEDOT nanocomposite. ${ }^{[25]}$ Zhou et al. synthesized novel yolk-shell S-PANI nanocomposite after heat treatment of the core-shell composite, as shown in Figure 6a and $b .{ }^{[26]}$ The void space between the sulfur particle and carbonized PANI shell was expected to effectively accommodate the volume change of sulfur during the lithiation/de-lithiation process, thereby preserving the structural integrity and enhancing the cycling performance. As a result, the electrode exhibited much-stabilized capacities with $765 \mathrm{mAh} \mathrm{g}^{-1}$ at a $0.2 \mathrm{C}$ and $628 \mathrm{mAh} \mathrm{g}^{-1}$ could be delivered at a $0.5 \mathrm{C}$ rate after 200 cycles, respectively.

In addition, the metals and relative compounds with well-defined nanostructures, such as $\mathrm{SiO}_{2},{ }^{[27]} \mathrm{TiO}_{2},{ }^{[28]} \mathrm{MnO}_{2},{ }^{[29]}$ porous $\mathrm{Ni}$ foam, ${ }^{[30]}$ have also been investigated as sulfur hosts for Li$\mathrm{S}$ batteries. Moreover, multi-phase composites, such as S@PEDOT/MnO ${ }_{2},{ }^{[31]}$ PEDOT:PSScoated CMK-3/sulfur, ${ }^{[32]}$ sulfur-infiltrated graphene-backboned mesoporous carbon nanosheets with PPy coating, ${ }^{[33]}$ Nafion-coated functionalized graphene sheets-sulfur (FGSS), ${ }^{[34]}$ have been further proposed to increase the contact area and wettability of the polymer network with the electrolyte, thus improving the electrochemical performance of Li-S batteries. Wu et al. modified the surface of as-prepared hollow carbon spheres/sulfur $(\mathrm{HCSs} / \mathrm{S})$ and graphene. ${ }^{[35]}$ Under the strong electrostatic interactions (Figure 6c) between the oppositely charged materials, the composite was formed self-assembly resulting in a layer-by-layer architecture (HCSs/S-LBL, Figure 6d and e). When served as the electrode, the HCSs/S-LBL electrode exhibited good cycling stability and high Coulombic efficiency. Even at the current density of $1 \mathrm{~A} \mathrm{~g} \mathrm{~g}^{-1}$, a stable 
reversible capacity of $575 \mathrm{mAh} \mathrm{g}^{-1}$ could still be achieved after 200 cycles (Figure 6f), indicating a strong capability to suppress the diffusion of polysulfides compared with the HCSs/S electrode.

(a)

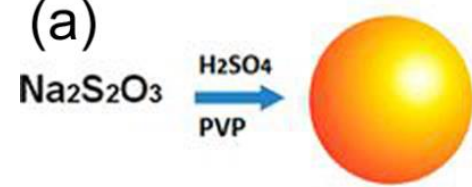

(b)

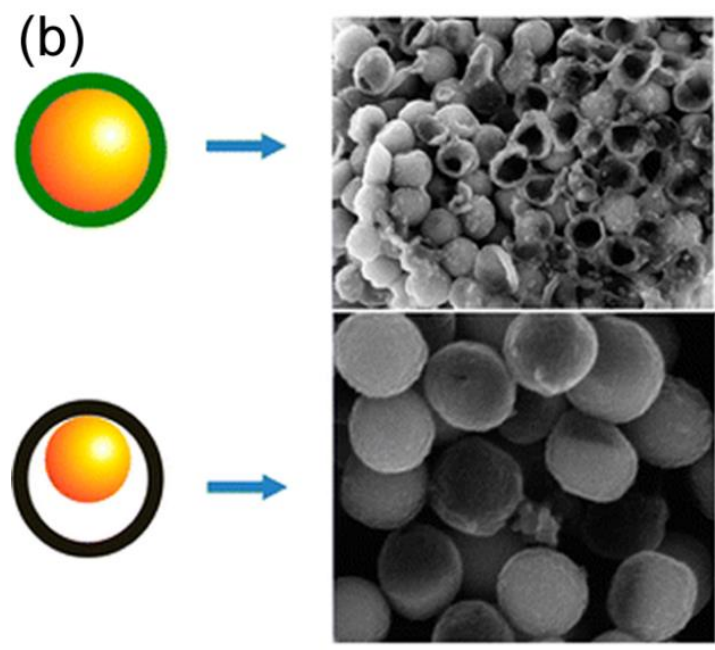

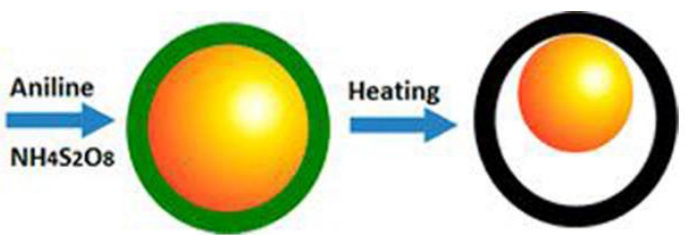
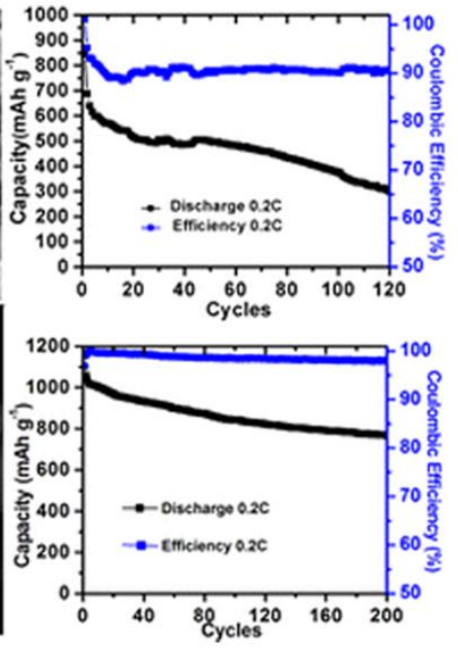

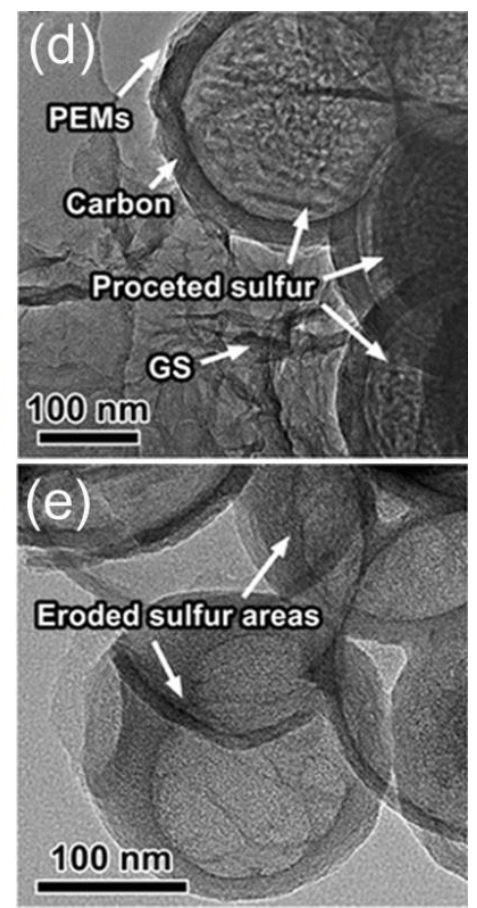

(c)
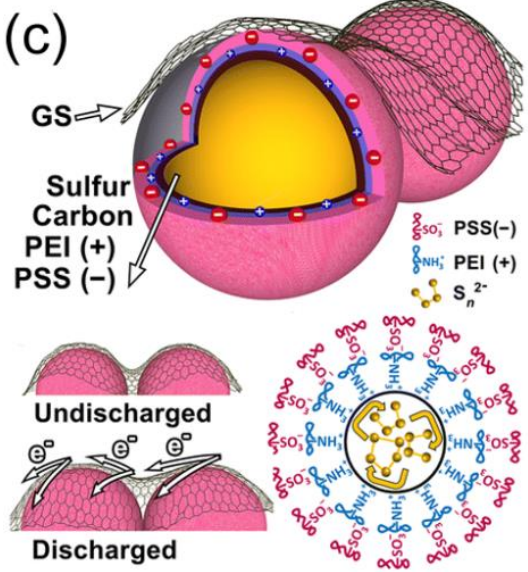

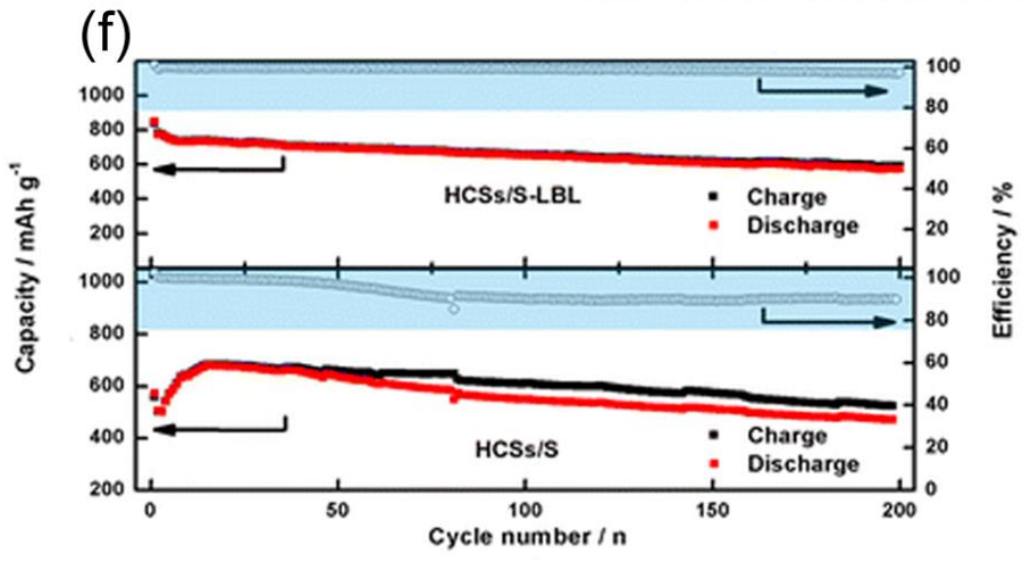

Figure 6 (a) Two-step synthesis route for S-PANI composite and (b) the schematic illustration of the structure effect of S-PANI on the long-term cycling performance. ${ }^{[26]}$ (c) The structure and functions of the PEMs and functionalized GS coating film with corresponding high-resolution TEM images of the HCSs/S-LBL (d) and HCSs/S composites after soaking in the $\mathrm{Li}_{2} \mathrm{~S}_{4}$ solutions

(e) and prolonged cycle performance at $1 \mathrm{~A} \mathrm{~g}^{-1}(\mathrm{f}) .{ }^{[35]}$ 
Table 1 Electrochemical performance of $\mathrm{S} / \mathrm{C}$ composites in the previous literature. (Note: $C_{1}$ : initial reversible capacity, $C_{2}$ : reversible capacity after $(x)$ cycles, $1 \mathrm{C}=1680 \mathrm{~mA} \mathrm{~g}^{-1}$.)

\begin{tabular}{|c|c|c|c|c|c|c|c|}
\hline $\begin{array}{l}\text { Cathodic } \\
\text { materials }\end{array}$ & $\begin{array}{c}\text { Sulfur } \\
\text { content }\end{array}$ & $\begin{array}{l}\text { Current } \\
\text { density }\end{array}$ & $\begin{array}{c}\mathrm{C}_{1} \\
\left(\mathrm{mAh}^{-1}\right)\end{array}$ & 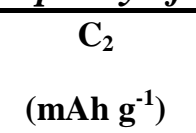 & $\begin{array}{l}\text { Capacity } \\
\text { retention }\end{array}$ & $\begin{array}{l}\text { Coulombic } \\
\text { efficiency }\end{array}$ & Reference \\
\hline $\begin{array}{l}\text { S-active } \\
\text { carbon }\end{array}$ & 30 wt. $\%$ & $0.3 \mathrm{~mA} \mathrm{~cm}^{-2}$ & 440 & $400(25)$ & $90.9 \%$ & $\sim 100 \%$ & {$[12 c]$} \\
\hline $\begin{array}{c}\mathrm{S}- \\
\text { micropores } \\
\text { carbon }\end{array}$ & 42 wt.\% & $400 \mathrm{~mA} \mathrm{~g}^{-1}$ & 820 & $\begin{array}{l}\sim 700 \\
(500)\end{array}$ & $85.4 \%$ & $\sim 100 \%$ & [12a] \\
\hline $\begin{array}{c}\mathrm{S} / \\
(\mathrm{CNT} @ \mathrm{MPC} \\
)\end{array}$ & 49 wt. $\%$ & $0.1 \mathrm{C}$ & 1269 & $\begin{array}{l}1142 \\
(200)\end{array}$ & $90 \%$ & $\sim 100 \%$ & [10] \\
\hline CMK-3/S & 70 wt.\% & $0.1 \mathrm{C}$ & 1005 & 800 (20) & $79.6 \%$ & NA & [3] \\
\hline $\begin{array}{l}\text { Carbon black } \\
\text { wrapped } \\
\text { sulfur }\end{array}$ & 75 wt. $\%$ & $0.05 \mathrm{C}$ & 1116 & 777 (50) & $69.6 \%$ & NA & {$[12 b]$} \\
\hline C@S & 70 wt.\% & $0.5 \mathrm{C}$ & 1076 & 974 (100) & $90.5 \%$ & $\begin{array}{l}\text { below } \\
95 \%\end{array}$ & [36] \\
\hline DHCS-S & 70 wt.\% & $0.1 \mathrm{C}$ & 935 & $690(100)$ & $73.8 \%$ & $\begin{array}{l}\text { below } \\
95 \%\end{array}$ & [37] \\
\hline $\begin{array}{c}\text { 950-CarbHS- } \\
\text { G/S }\end{array}$ & 54 wt. $\%$ & $1 \mathrm{C}$ & 1000 & $700(50)$ & $70 \%$ & NA & [4] \\
\hline $\begin{array}{l}\text { Nafion- } \\
\text { coated } \\
\text { FGSS }\end{array}$ & 72 wt.\% & $0.1 \mathrm{C}$ & 950 & $754(50)$ & $79.4 \%$ & NA & [34] \\
\hline S-PPy & 63 wt.\% & $0.2 \mathrm{C}$ & 739 & $634(50)$ & $85.7 \%$ & 90\% & [12e] \\
\hline $\begin{array}{c}\text { S- } \\
\text { PAAMPSA }\end{array}$ & 75 wt. $\%$ & $0.1 \mathrm{C}$ & 783.1 & $\sim 650(50)$ & $83.0 \%$ & $88 \%$ & [38] \\
\hline $\begin{array}{c}\text { S-Pani } \\
\text { core-shell }\end{array}$ & 82 wt. $\%$ & $0.2 \mathrm{C}$ & $\sim 700$ & $280(125)$ & $\sim 40 \%$ & $\sim 90 \%$ & [26] \\
\hline $\begin{array}{l}\text { CMK- } \\
\text { 3/sulfur }\end{array}$ & 50 wt. $\%$ & $0.2 \mathrm{C}$ & 1051 & $600(150)$ & $52.6 \%$ & $92-94 \%$ & [32] \\
\hline ACF-S & $\begin{array}{c}6.5 \mathrm{mg} \\
\mathrm{cm}^{-2}\end{array}$ & $150 \mathrm{~mA} \mathrm{~g}^{-1}$ & 1050 & 800 (80) & $76.2 \%$ & $95 \%$ & [39] \\
\hline $\mathrm{S} / \mathrm{C}$ & 40 wt. $\%$ & $100 \mathrm{~mA} \mathrm{~g}^{-1}$ & 1200 & $720(100)$ & $60 \%$ & $\sim 100 \%$ & [40] \\
\hline
\end{tabular}




\subsection{Sulfur immobilization by "chemical anchors"}

Although the physically composite sulfur with conductive host materials can trap the dissolved polysulfides in some degree, however, high soluble LiPSs can diffuse through these open pores within the physical barrier since the weak interactions between host materials and polysulfides $^{[41]}$ can only hold for a short-term and therefore, the escape of polysulfides cannot be eliminated. ${ }^{[14 c, 42]}$ That's why most of the published literature shows capacity degradation with low Coulombic efficiency based on this physical absorption mechanism, especially when tested more than 100 cycles. Some electrochemical performances of previous work on S/C composites are summarized in Table 1. It can be observed that the capacity retention and Coulombic efficiency (mostly below 95\%) are still unsatisfied by physically compositing sulfur with corresponding host materials. To completely prevent the diffusion of polysulfides and their "shuttle effect", a new type of cathode materials named "sulfurized carbon" has been effectively investigated in recent years, i.e., sulfur species are covalently bonded to the host materials, such as carbonaceous materials by reacting with the functional groups on the surface, or polymer backbone by grafting onto the conjugated carbon. ${ }^{[43]}$ This is the so-called "chemical adsorption".

Carbonaceous materials usually contain surface functional groups such as $\mathrm{C}-\mathrm{H},-\mathrm{OH}$, $>\mathrm{C}=\mathrm{O},-\mathrm{C}(=\mathrm{O}) \mathrm{OH}$, and $>\mathrm{C}=\mathrm{C}<$, which shows great potential in binding sulfur on the surfaces of carbon during heat treatment. Heteroatoms doped carbonaceous materials, such as sulfur-- ${ }^{[43]}[44]$ oxygen-, ${ }^{[45]}$ and nitrogen-doped, ${ }^{[46]}$ or functional groups modified carbon host have been sufficiently investigated. ${ }^{[47]}$ Another major method is chemically catenating sulfur into functional conductive polymers since polymer materials are usually synthetic tailorability, i.e., their

electrochemical and physical properties can be easily tuned by surfactant modification. ${ }^{[48]}$ In these organosulfur polymers, sulfur can be covalently bound to a polymeric backbone and form 
reversible cleavage of disulfide bridges. Extensive efforts have targeted the synthesis of conductive polymers as a backbone bonding with sulfur, such as PAN, ${ }^{[22 a, 49]}$ disulfide chain on PANi, ${ }^{[50]}$ and polythiophene. ${ }^{[51]}$ The synthetic tailorability of polymer materials results in prosperous research on incorporating sulfur into the polymer chains. The sulfur cathodes based on chemical bonding between sulfur and polymer provide new trends and ideas for avenues of further research to advanced Li-S battery technologies. However, these polymers usually show lower electronic conductivity than carbonaceous materials and the compatibility with electrolytes need to be highly considered. Otherwise, the side reactions with electrolytes can cause a significant decrease in the Columbic efficiency.

Another alternative way to create much stronger interaction between $S$ and the host materials is by the introduction of polar metal compounds. Transition metals oxides and sulfides have been demonstrated as effective polar cathode materials for adsorbing polysulfides in Li-S batteries. The strong polar surface of metal oxides due to the oxidation state of $\mathrm{O}^{2-}$ results in polar-polar chemical interactions with polysulfides. As to metal sulfides, the strong bonds between metal and sulfur allow the $\mathrm{S}$ in metal sulfides to form $\mathrm{Li}_{2} \mathrm{~S}$ and metal without forming soluble high-order polysulfide during the lithiation process. In addition, such metal sulfides show intrinsic sulfiphilic towards polysulfides and can enhance the redox kinetics of sulfur species by electrocatalysis with increasing the tap density of electrode, sulfur utilization, and long life span. However, the relatively lower theoretical capacity compared with elemental sulfur of these metal sulfides greatly limit the potential for commercial application. At present, metal oxides and sulfides could be described as additives or host materials for solid sulfur cathodes without sacrificing the loading of sulfur. Great progress on the application of these type materials in anchoring polysulfides for Li-S batteries is reviewed in the following section. 


\subsubsection{Metal/metal oxides as host materials}

Zheng et al. reported a copper-stabilized sulfur-microporous carbon (MC) cathode for Li-S batteries, ${ }^{[42]}$ which was prepared by ultrasonic-assisted multiple wetness impregnations and synchro-dry technique (Figure 7a) with $\mathrm{Cu}$ nanoparticles as an additive in $\mathrm{MC}$. The $\mathrm{Cu}$ nanoparticles loading in $\mathrm{MC}$ could effectively stabilize the heavy $\mathrm{S}$ through $\mathrm{Cu}-\mathrm{S}$ bonding, as confirmed by XRD patterns of MC-Cu-S in Figure 7b. The new peaks can be ascribed to the formation of $\mathrm{CuS}_{\mathrm{x}}$ compounds. The unique structural $\mathrm{MC}-\mathrm{Cu}-\mathrm{S}$ composite cathode showed a high Coulombic efficiency close to $100 \%$, maintained capacities of around $630 \mathrm{mAh} \mathrm{g}^{-1}$ at the current density of $100 \mathrm{~mA} \mathrm{~g}^{-1}$ with progressive cycling up to 500 cycles, and delivered a capacity of $200 \mathrm{mAh} \mathrm{g}^{-1}$ even at a high current density of $10.0 \mathrm{~A} \mathrm{~g}^{-1}$. The outstanding performance of MC-Cu-S cathode is because i) Cu-polysulfide clusters formed through strong interaction between $\mathrm{Cu}$ and $\mathrm{S}$; ii) electronic conductivity can be improved by introducing nano-sized $\mathrm{Cu}$ particles; iii) MC host provides free space to accommodate the volume change of S. The results represented that only a small amount of metal nanoparticle anchored in MC could substantially stabilize the $\mathrm{S}$ cathode, increase the $\mathrm{S}$ loadings and improve the cycling stability. Ni et al. just have recently reported a $\gamma-\mathrm{MnO}_{2}$ coated solid sulfur nanocomposite with core-shell structure through the redox reaction between $\mathrm{KMnO}_{4}$ and $\mathrm{MnSO}_{4}$, and deeply investigated interaction mechanisms of $\gamma-\mathrm{MnO}_{2}$ and polysulfides in the rechargeable Li-S cells. ${ }^{[52]}$ The nanorods $\gamma-\mathrm{MnO}_{2}$ shell could physically encapsulate sulfur/polysulfides and buffer the volume expansion during lithiation. More importantly, the $\gamma-\mathrm{MnO}_{2}$ host would chemically trap polysulfide through "Wacknroder reaction", as illustrated in Figure 7c. The soluble polysulfides on the surface of $\gamma$ $\mathrm{MnO}_{2}$ were oxidized into $\mathrm{SO}_{x}$ species via the transfer of oxygen atoms from the $\gamma-\mathrm{MnO}_{2}$ surface to a polysulfide molecule, which was accompanied by the phase transformation from $\gamma-\mathrm{MnO}_{2}$ to 
$\mathrm{Mn}_{3} \mathrm{O}_{4}$. Subsequently, the insoluble thiosulfate $\left[\mathrm{S}_{2} \mathrm{O}_{3}\right]^{2-}$ could catenate to sulfur chains of polysulfide in solution to create the polythionate $\left[\mathrm{SO}_{3} \mathrm{~S}_{y} \mathrm{SO}_{3}\right]^{2-}$ and a short-chain polysulfide $\mathrm{S}_{y}{ }^{2-}$ $(x \geq 4, y<3)$ by an internal disproportionation reaction. Nazar and co-workers also presented ultrathin $\mathrm{MnO}_{2}$ nanosheets as a host material and confirmed the redox reaction between polysulfides and $\mathrm{MnO}_{2}$ by XPS studies. ${ }^{[53]}$ The mechanism of this polythionate complex formation can be described below:

$$
\left[\mathrm{S}_{2} \mathrm{O}_{3}\right]^{2-}+S_{x}^{2-} \rightarrow\left[\mathrm{SO}_{3} S_{x-y} \mathrm{SO}_{3}\right]^{2-}+S_{y}^{2-}(x \geq 4, y<3)
$$

Various other oxides with different morphologies have also been investigated as effective host materials, such as nano-sized $\mathrm{Mg}_{0.6} \mathrm{Ni}_{0.4} \mathrm{O},{ }^{[54]}$ hollow $\mathrm{TiO}_{2}$-webbed carbon nanotubes, ${ }^{[55]} \mathrm{TiO}_{2}$ nanosheets, ${ }^{[28 a]}$ porous $\mathrm{Ti}_{4} \mathrm{O}_{7}$ nanoparticles, ${ }^{[56]} \mathrm{V}_{2} \mathrm{O}_{3}$ modified porous carbon microsphere, ${ }^{[57]}$ $\mathrm{Si} / \mathrm{SiO}_{\mathrm{x}}$ doped carbon materials. ${ }^{[27,58]}$
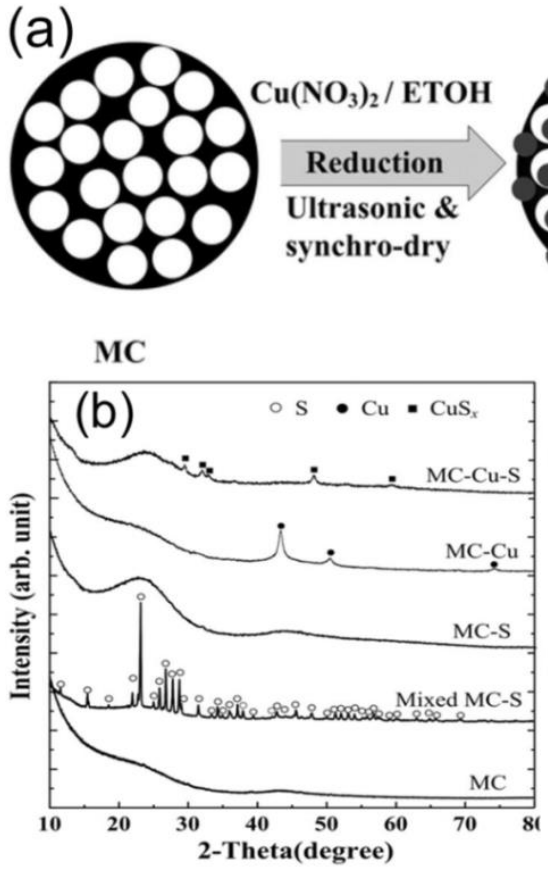

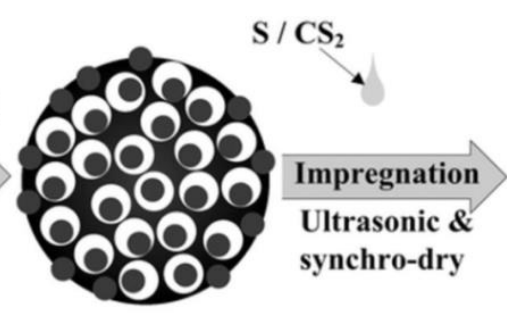

$\mathrm{MC}-\mathrm{Cu}$
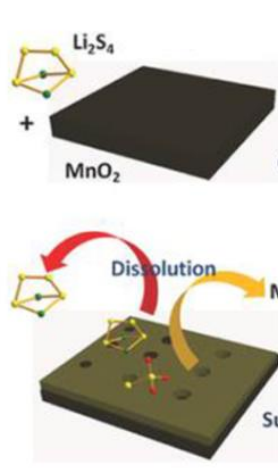

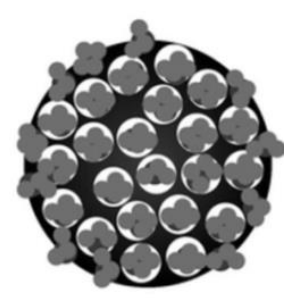

MC-Cu-S

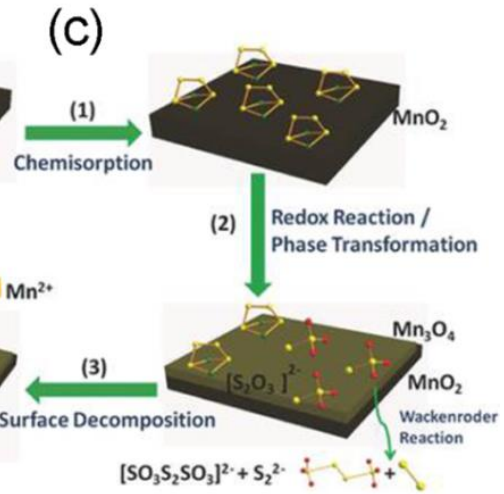

Figure 7 (a) the preparation process for MC-Cu-S composite, (b) XRD patterns of MC, Mixed MC-S, MC-S, MC-Cu, and MC-Cu-S. ${ }^{[42]}$ c) The proposed interaction mechanisms of polysulfide and $\gamma-\mathrm{MnO}_{2}$ on the surface. ${ }^{[52]}$ 
2.2.2 Metal sulfides as the host or additive materials.

Metal sulfides (MSs) usually widely exist in nature and possess better electrical conductivity, mechanical and thermal stability than their corresponding metal oxides, endowing them being potential electrode materials for LIBs. With their strong sulfiphilic property to sulfur-containing species, great progress has been made in metal sulfides with different nanostructures. Some representative researches related to the design of smart architecture metal sulfides will be reviewed here. As the same as metal oxides, MSs can also be used as host materials or "chemical anchor" for polysulfides. As the most abundant sulfur mineral, $\mathrm{FeS}_{2}$ has been sufficiently investigated for absorbing lithium polysulfides. ${ }^{[59]}$ Tan et al. reported a novel cathode material with core nano-FeS $\mathrm{F}_{2}$ coated by the shell of $\mathrm{N}$-doped graphene. ${ }^{[60]}$ As shown in Figure 8a, Prussian blue was adopted as a precursor, the $\mathrm{FeS}_{2} @ \mathrm{~N}$-graphene was obtained after pyrolyzation, oxidation, and sulfurization process. The core-shell structure was further confirmed by TEM images in Figure $8 \mathrm{~b}$ and $\mathrm{c}$. The corresponding mechanism can be illustrated in Figure $8 \mathrm{~d}$ and e. During the discharge process, $\mathrm{FeS}_{\mathrm{y}}$ would transform to $\mathrm{Fe}$ and $\mathrm{Li}_{2} \mathrm{~S}$. Fe nanoparticles formed a conductive framework allowing the reversible reaction of $\mathrm{Li}_{2} \mathrm{~S}$ to proceed with fast kinetics. In addition, the $\mathrm{N}-$ doped graphene shells tightly attached to the nano-FeS 2 particles could enhance the structural stability during the charge-discharge process. As a result, the $\mathrm{FeS}_{2} @ \mathrm{~N}$-graphene still had a very high capacity of $402 \mathrm{mAh} \mathrm{g}^{-1}$ after 400 cycles at the current density of $0.5 \mathrm{~A} \mathrm{~g}^{-1}$ with the Coulombic efficiency almost to $100 \%$.

Generally, for MSs ( $\mathrm{M}=\mathrm{Mn}, \mathrm{Fe}, \mathrm{Co}, \mathrm{Ni}$, and $\mathrm{Cu}$ ), the reaction with lithium could be described as a "conversion type" mechanism, as shown in Equation $4 .{ }^{[61]}$ Based on this mechanism, other metal sulfides such as $\mathrm{CoS}_{2} / \mathrm{rGO} / \mathrm{MWCNTs}$ composites, ${ }^{[62]} \mathrm{CuS}$-based composites, ${ }^{[63]} \mathrm{NiS}$ nanobelts, ${ }^{[64]}$ nanoflakes, ${ }^{[65]}$ nanorods, ${ }^{[66]}$ hollow spheres, ${ }^{[67]}$ have also been explored as the cathode materials in 
energy storage. Although it is widely known that metal oxides/sulfides host materials exhibit lower surface area, pore-volume, and electrical conductivity resulting in a low areal sulfur loading as well as gravimetric energy, their strong polar-polar interaction with polysulfides still make them be one of the most promising candidates as "host materials". In addition, due to the intrinsic conversion mechanism, the compact metal oxides/sulfides particles will transform to polycrystalline nanoparticles during the lithiation process. The resultant polycrystalline significantly increases the surface area, which may provide more active sites as a chemical anchor. Moreover, the nanometal particles formed after lithiation (based on Eqn. 4) build a conductive framework, which will help to promote the reversible reaction of polysulfides. The nanometal particles uniformly embedded in the $\mathrm{Li}_{2} \mathrm{O}$ or $\mathrm{Li}_{2} \mathrm{~S}$ matrix decreases the $\mathrm{Li}^{+}$diffusion pathway and provide more electron transfer channels, thus fast kinetics of reversible conversion from polysulfides to metal oxides/sulfides can be expected. Based on this point, the polysulfides may have less chance to diffuse to the anode side and can be effectively confined to the metal oxides/sulfides host.

$$
M S_{x}+2 x L^{+}+2 x e^{-} \leftrightarrow x L i_{2} S+M
$$


(a)

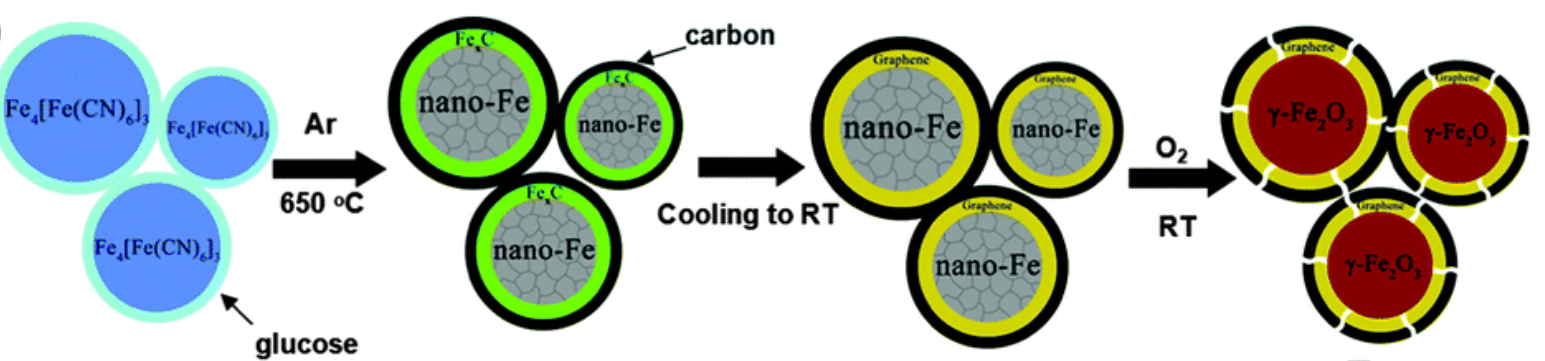

glucose
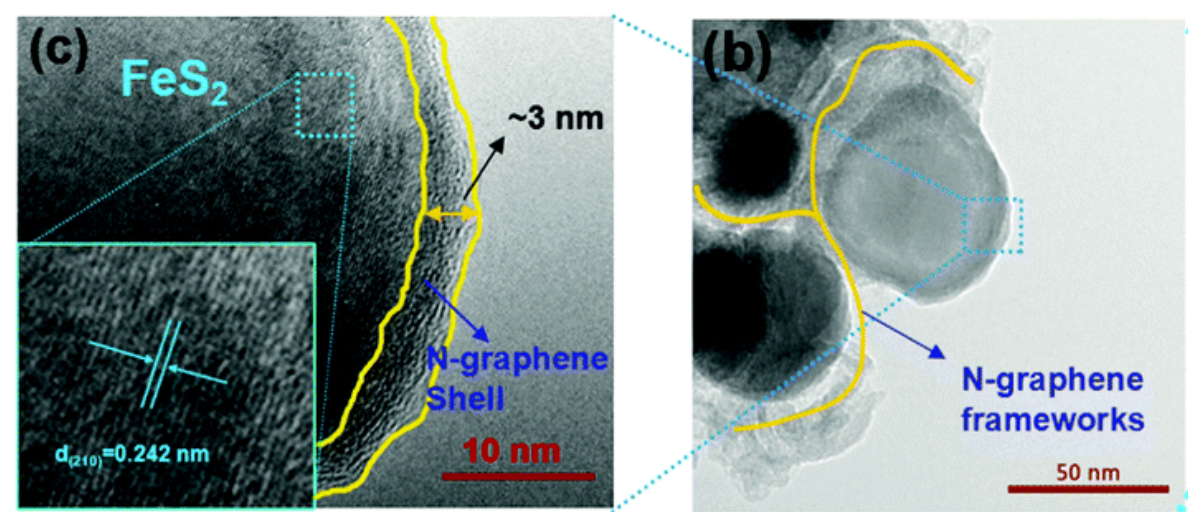

Sealed in a tube with $\mathrm{S}_{8}$

$650 \circ \mathrm{C}$

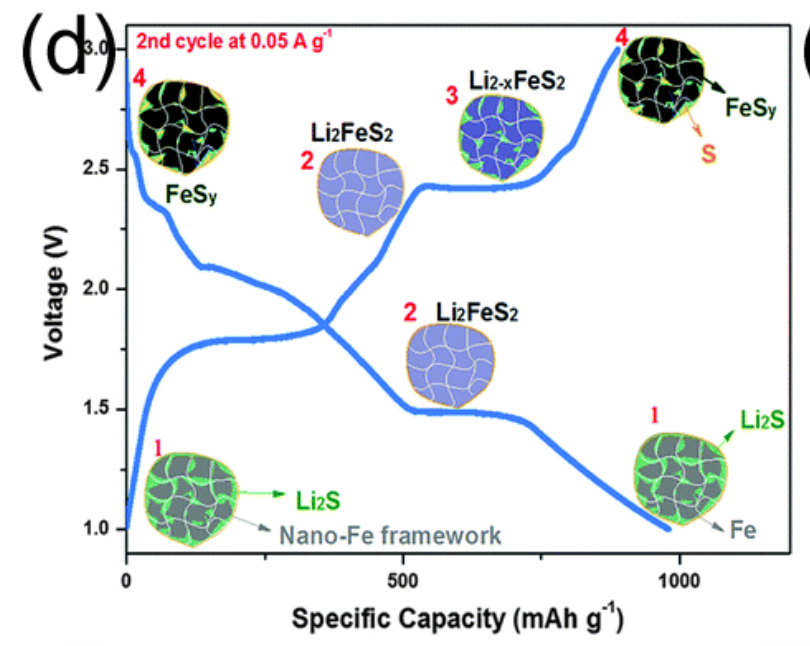

(e) state 1

State 2.1

State 2.2
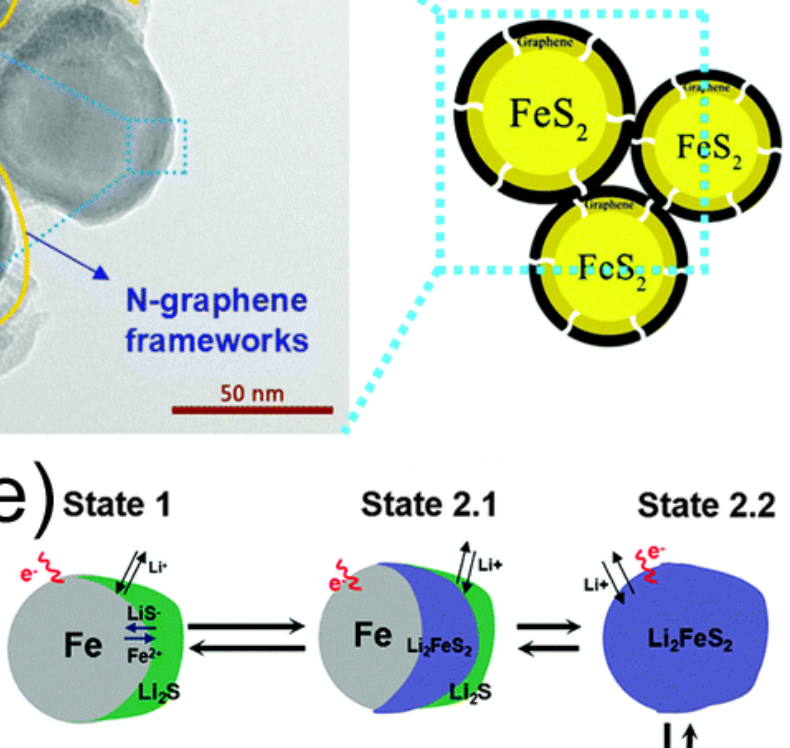

Cycling Procedure

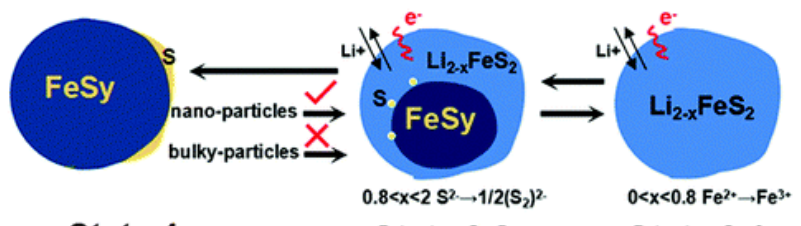

State 4

State 3.2

State 3.1

Figure 8 (a) Brief illustration of the fabrication of $\mathrm{FeS}_{2} @ \mathrm{~N}$-graphene (RT: room temperature), (b, c) TEM image, and HR-TEM image of $\mathrm{FeS}_{2} @ \mathrm{~N}$-graphene particles, (d, e) schematic diagrams for the reaction mechanism. ${ }^{[60]}$

\subsubsection{Metal-Organic Frameworks (MOFs).}

With higher porosity, larger specific surface areas, multiformity of structures and functions than traditional inorganic porous materials, MOFs have attracted people's attention either as a 
precursor or surfactant applied in supercapacitor and LIBs. ${ }^{[68]}$ One of the most common sulfur hosts is MOF-derived carbon materials. The large surface area, fitted pore size, and unique morphologies from MOFs make them very suitable for sulfur host materials. ${ }^{[69]}$ However, research efforts have focused more on the polysulfides directly chemically interacting with MOF hosts based on the Lewis acid-base interaction mechanism between MOF and polysulfides. ${ }^{[70]}$ Lim et al. reported a chemically anchored polymer-coated (CAPC) sulfur electrode through chemical bonding by coordinated $\mathrm{Cu}$ ions and crosslinking to improve cyclability for $\mathrm{Li} / \mathrm{S}$ batteries. ${ }^{[71]}$ Figure 9a shows a schematic illustration of the preparation of the sulfur-carbon black composite electrode anchored with $\mathrm{Cu}(\mathrm{I}) \mathrm{Br}$ and 4-vinylpyridine initiator, followed by surface-induced thermal cross-linking polymerization using a solution containing 12 wt. \% ethylene glycol diacrylate (EGD) crosslinker in $\mathrm{CH}_{3} \mathrm{CN}$. For comparison, a cross-linked polymer-coated sulfur electrode was also prepared under the same conditions, but without the use of $\mathrm{Cu}(\mathrm{I}) \mathrm{Br}$ additive. The anchored copper-4 vinylpyridine complex on the sulfur-carbon composite electrode was analyzed by both FT-IR (Figure 9b) and Raman spectroscopic (Figure 9c) methods. Even after 100 cycles, more than $85 \%$ of the specific capacity was retained for this chemically anchored polymer-coated sulfur electrode, and much lower capacities were obtained for both the bare sulfur electrode and for the electrode in which the polymer coating only physically covered the S rather than chemically bonded with it. 

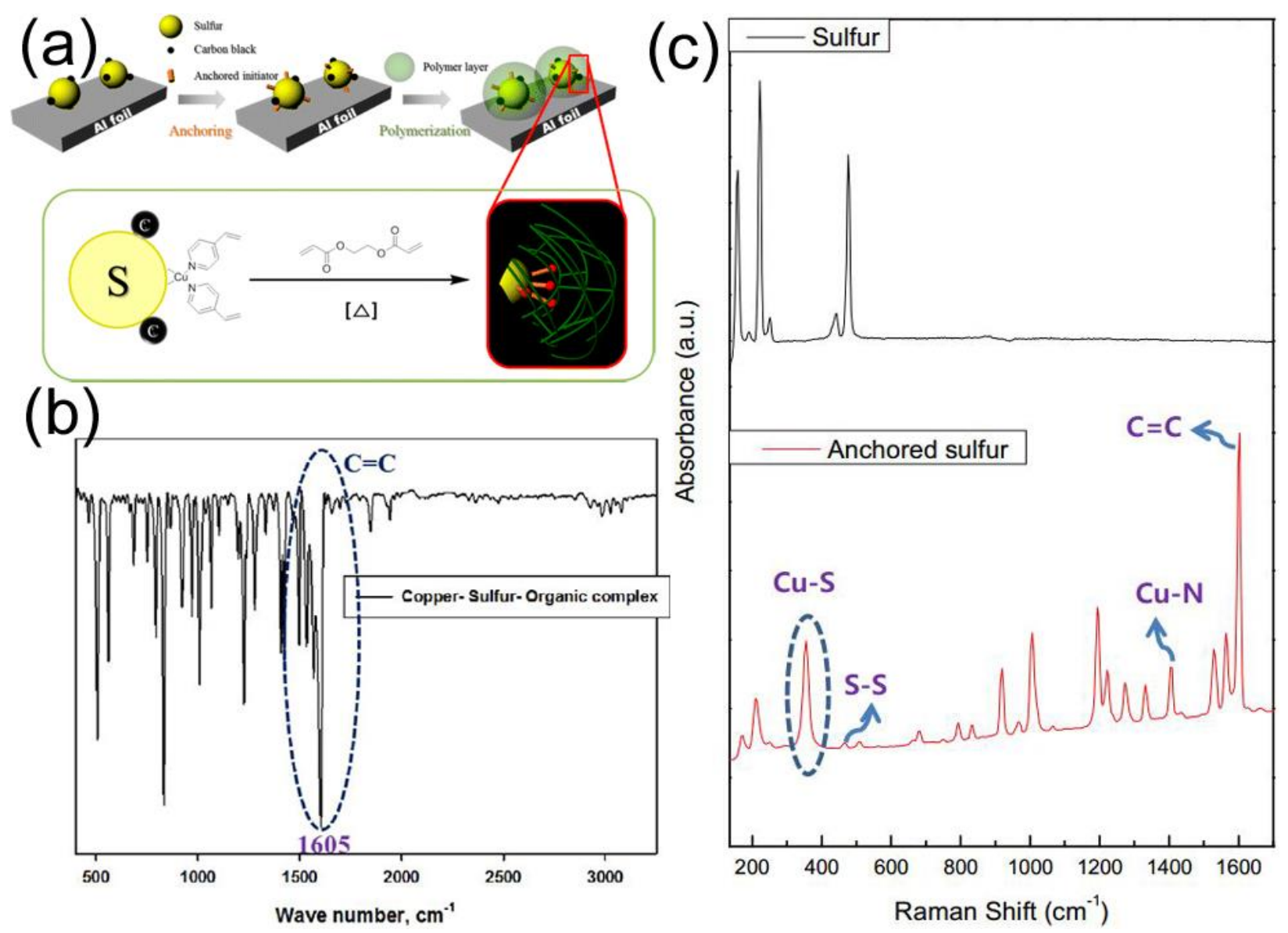

Figure 9 (a) Schematic illustration for the preparation of the $\mathrm{Cu}$-anchored cross-linked polymercoated sulfur electrode, (b) FT-IR spectra of the chemically anchored polymer-coated (CAPC) sulfur, (c) Raman spectra of the pristine sulfur and the $\mathrm{Cu}$-vinyl pyridine-anchored sulfur. ${ }^{\text {[71] }}$

Metal-organic polyhedron (MOP) has also been attracting more and more research team's attention for electrochemical energy storage. Bai et al. developed novel hybrids by employing three different MOPs, i.e., ZIF-8 (Zn), ZIF-67 (Co), and HKUST-1 (Cu), to encapsulate PVPfunctionalized sulfur nanoparticles. ${ }^{[72]}$ The PVP as a surfactant could trap the polysulfides and MOPs coating further physically and chemically adsorbed the polysulfides. As illustrated in Figure 10a, PVP can not only stabilize the sulfur nanoparticles in polar solvents but also control the shape and size of the particles. After added the MOPs solutions and stirred for $24 \mathrm{~h}$, different 
S@MOP hybrids with uniform particle size could be obtained. As seen in Figure 10b, both TEM and FESEM images show that the uniformity of the morphology of the S@MOP composites. The elemental mapping further reveals the uniform distribution of sulfur. When tested the electrochemical performance for all these three electrodes, the S@HKUST-1 showed best cycle performance and rate capability, i.e., Cu-MOF showed the best chemical adsorption compared with others, which coincides with the results reported by Xiao's group. ${ }^{[70]}$ Similarly, Mao and co-workers developed a promising MOF-based thin film electrode for Li-S batteries. ${ }^{[73]}$ HKUST1, MOF-5, and ZIF-8 were employed to investigate the pore size effect on Li-S performance. The prepared CNTs interpenetrated MOFs thin film provided appropriate pores to well confine the sulfur particles. In addition, the negatively charged CNTs around the MOFs particles could effectively accommodate the large volume change and facilitate fast electron/ $/ \mathrm{Li}^{+}$transport (Figure 10c). Due to this unique hierarchical nanoporous and macroporous structure, the designed MOFs-based electrodes exhibited remarkable electrochemical performance as illustrated in Figure 10d and e. A high reversible capacity of $\sim 450 \mathrm{mAh} \mathrm{g}^{-1}$ for the S@HKUST1/CNT electrode can be achieved even at a high rate of $10 \mathrm{C}$. And when cycled at $0.2 \mathrm{C}$, the S@HKUST-1/CNT electrode can maintain a capacity of nearly $1000 \mathrm{mAh} \mathrm{g}^{-1}$ after 50 cycles. 


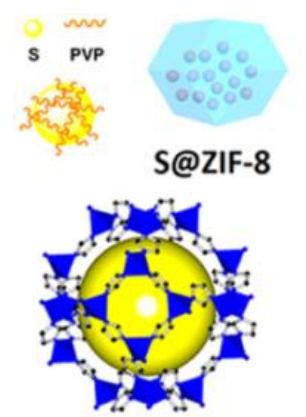

ZIF-8
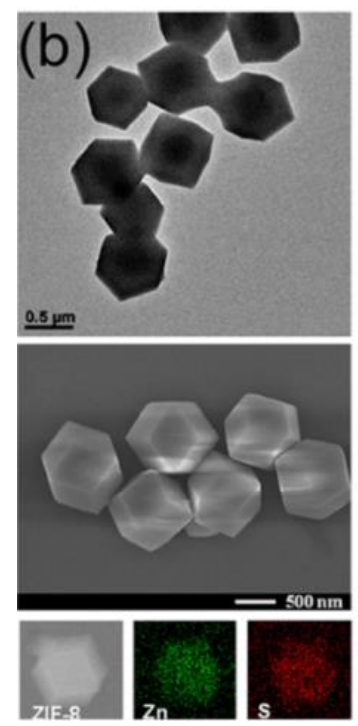

(a)

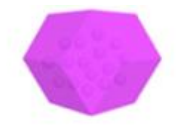

S@ZIF-67

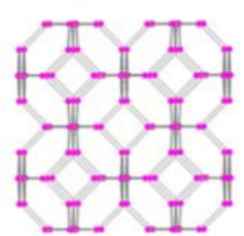

ZIF-67
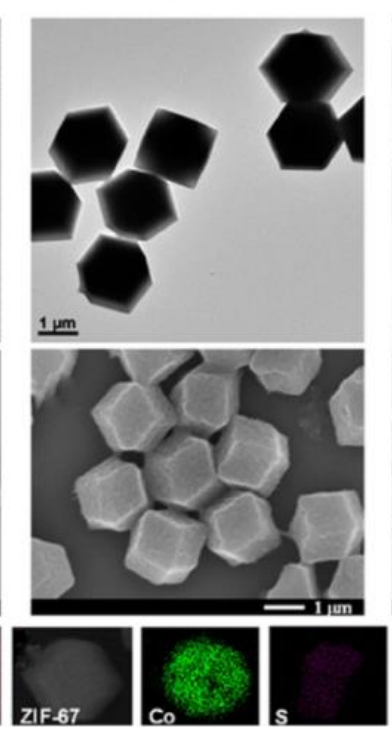

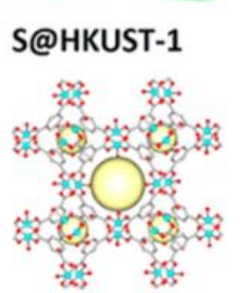

HKUST-1
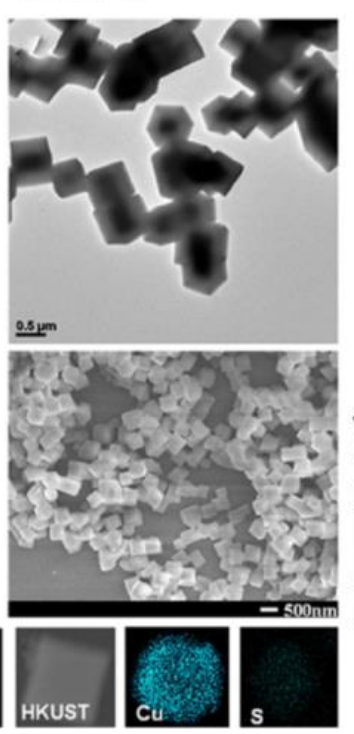
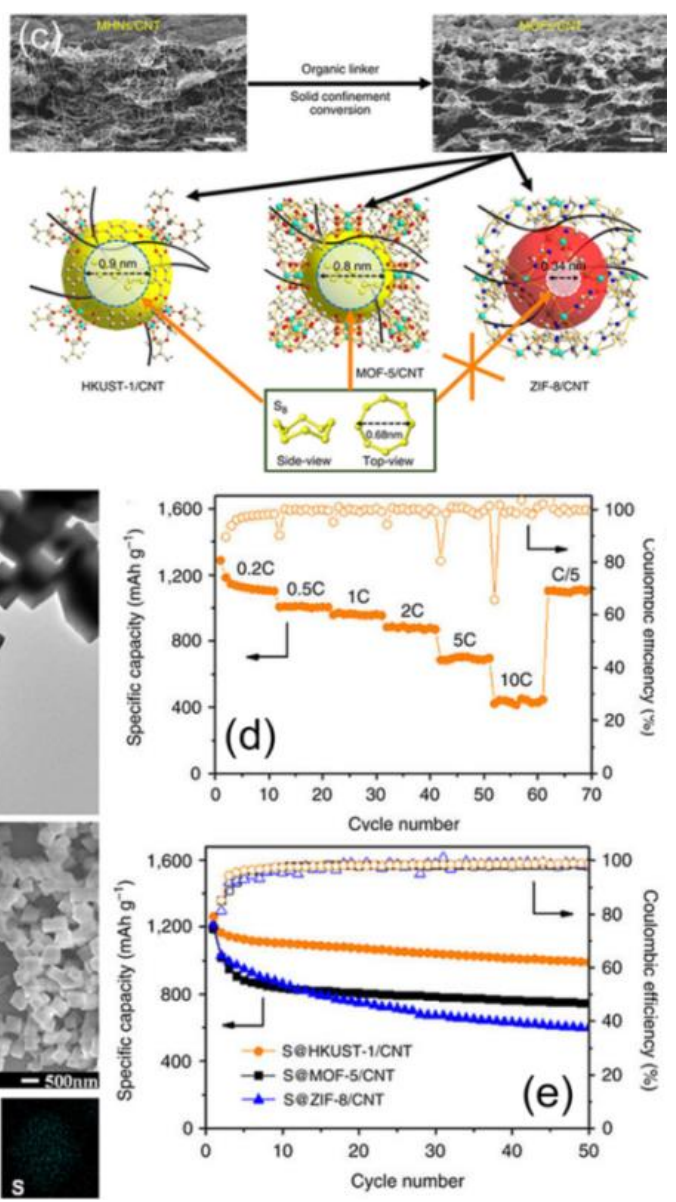

Figure 10 (a) Schematic illustration of different MOF encapsulated sulfur, (b) TEM and SEM images of S@ZIF-8, S@ZIF-67, and S@HKUST-1 hybrids with corresponding elemental mapping as marked. ${ }^{[72]}$ (c) Synthesis of $\mathrm{S}_{8}$ loaded MOFs/CNT composite thin films, d) rate performances of S@HKUST-1/CNT electrode and e) the cycling performances of S@HKUST1/CNT, S@MOF-5/CNT, and S@ZIF-8/CNT electrodes, respectively. ${ }^{[73]}$

According to the previous discussion, we have summarized different types of "host material" in cathode electrodes based on the "chemical adsorption" mechanism. For easier comparison, we list the S-cathode materials with chemical bond effects, and the corresponding electrochemical performances, characterization methods in Table 2. Obviously, the cathode materials containing these "chemical anchors" show much better electrochemical performances, especially for the 
remarkable Coulombic efficiency and long-term cycle stability. Most of the electrodes could maintain high reversible capacity after hundreds of cycles with Coulombic efficiency close to $100 \%$. According to the results listed in Table 2, most reported characterization methods are found to be related to C-S bonding, indicating the carbonaceous materials are still the most widely used host material. New characterization methodslare expected to be developed for investigating the type of chemical bonding. It should be also noticed that the sulfur loading listed in Table 2 is the percentage of contents of the composites. The total sulfur loading should be even lower in the cathode, which is still far away for the practical applications of Li-S batteries. 
Table 2 Summary of electrochemical performance of the reported sulfur electrodes.

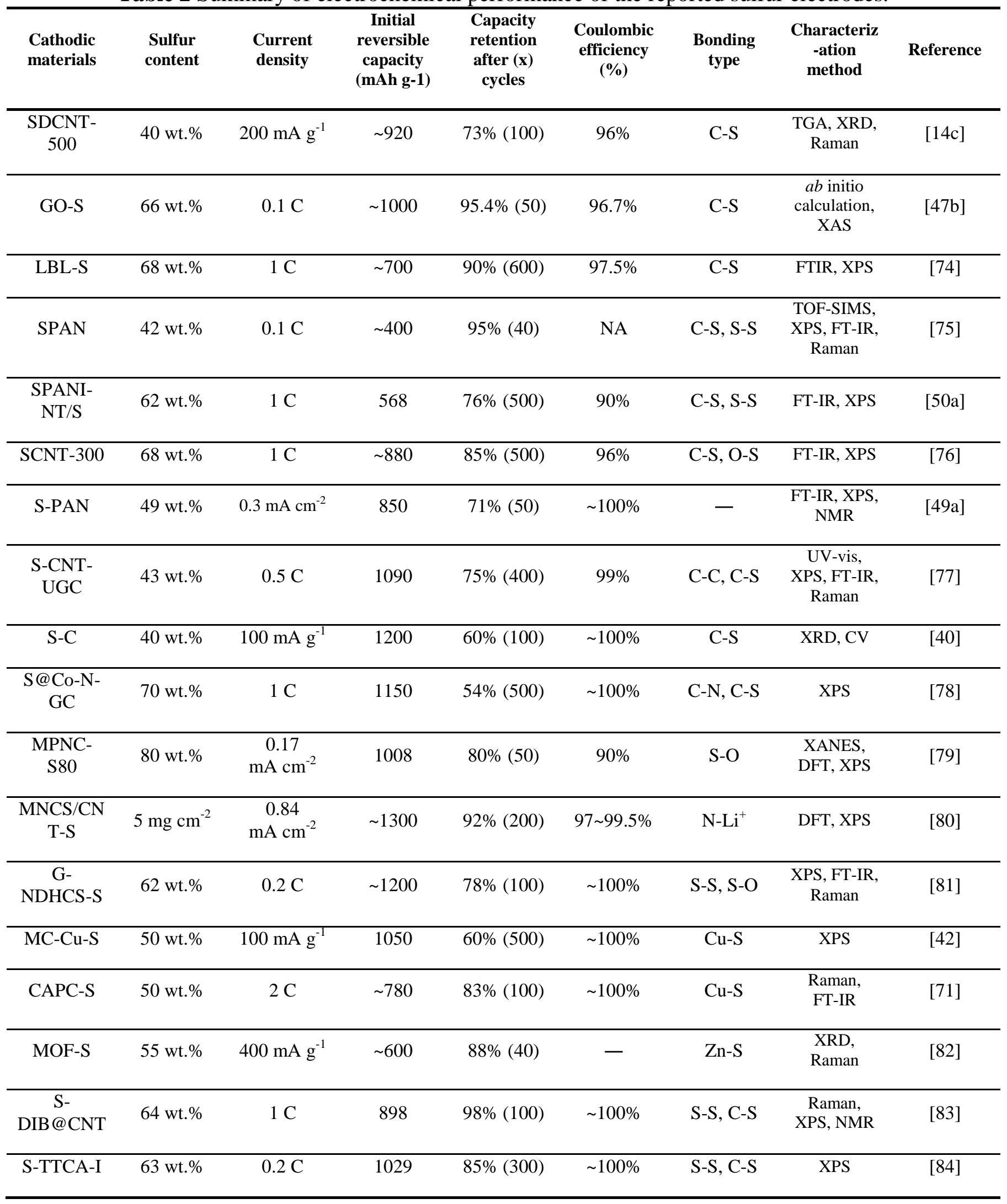


To further investigate the possible factors which will influence the Li-S performance, Table 3 has also been added for better comparison. The binding energies between lithium polysulfides and host materials based on different chemical anchors are compared as well as the morphology, structure, particle size, surface area of the host materials. Basically, the electrochemical performance cannot be only determined by the binding energy. To select the best one among these materials is difficult only based on the binding energy since the electrochemical performance is a result of the synergistic effect of all the factors mentioned above. In addition, the binding energy between the host material and polysulfides is significantly affected by the functional groups on the host materials. ${ }^{[47 b, 85]}$ As indicated by Cheng's group, ${ }^{[86]}$ the DFT calculation results show that the hydroxyl/epoxide on the graphene will lead to different binding energy to the polysulfides even though both of them anchoring the polysulfides through the "O-S" bonding. Due to the limitations of characterization methods and theoretical models for calculation, the data of binding energy for a specific bonding type is variable and poor. The data for binding energy presented in Table 3 is based on the interaction between different surfactants, substrates, and polysulfides. For example, the N-containing function groups in the polymer ${ }^{[87]}$ show much different binding energy from that of $\mathrm{N}$-doped carbonaceous materials. ${ }^{[88]}$ Furthermore, different types of $\mathrm{N}$ atoms on the graphene (Pyrrole N, Pyridine $\mathrm{N}$ ) will yield to different binding energy to different polysulfides (from 0.25 to $2.10 \mathrm{eV}$ ) according to the $a b$ initio calculation results reported by Zhang's group ${ }^{[89]}$ However, we can still make a qualitative analysis based on the polar-polar interaction mechanism. Basically, N, P, and B-doped carbon show lower binding energy to polysulfides based on the formation of " $\mathrm{Li}$ - bond" ${ }^{[90]}$ than the "CS" and "O-S" based compounds, which directly anchor sulfur through a covalent bond. However, the latter usually needs a higher temperature to promote this process thus resulting in a low sulfur 
loading in the composites. In addition, the electronic conductivity of sulfurized carbon will decrease. Metal oxides/sulfides show even stronger binding energy than the heteroatoms doped carbonaceous materials, and metal oxides seem to have higher binding energy than the corresponding sulfides. ${ }^{[91]}$ Zhong et al. investigated the surface chemical interactions between LiPSs and metal oxides, and made a conclusion based on their XPS results and DFT calculation that the strength of interaction with LiPSs increased with an increasing atomic number of the transition metal oxides $\left(\mathrm{Mn}_{3} \mathrm{O}_{4}<\mathrm{Fe}_{2} \mathrm{O}_{3}<\mathrm{Co}_{3} \mathrm{O}_{4}\right) \cdot{ }^{[91 \mathrm{a}]}$ More importantly, they found that $\mathrm{Li}-\mathrm{O}$ interactions were more predominant due to the stronger electronegativity of oxygen. Zhang's group claimed that VS exhibited the strongest interaction with $\mathrm{Li}_{2} \mathrm{~S}$ after explored systematically the first-row TMSs. But similarly, they also found that Li-O bonds were more likely formed in TMOs while Li-S bonds were dominated in TMSs. ${ }^{[92]}$ In a word, there is still a long way to understand the underlying mechanism between the "chemical anchor" and polysulfides. New characterization methods and more reasonable theoretical approaches are needed to predict the binding energy and guide the design of materials.

Morphology and structure of the composites also play a critical role in the Li-S performance. The results randomly searched from the published literature as summarized in Table 3 indicate that microporous and mesoporous structure with specific surface area usually larger than $500 \mathrm{~m}^{2}$ $\mathrm{g}^{-1}$ can exhibit better electrochemical performance. Recently, much attention has been paid to design hierarchical structured micro-mesoporous host materials with multi-function to both physically and chemically adsorb the polysulfides and increase the sulfur loading. The large surface area can provide more chemical anchor active sites. That why most metal oxides/sulfides act as additives and have been coupled with conductive polymers or porous carbon materials to provide the best service as sulfur electrodes. 
Table 3 Summary of factors that may affect the electrochemical performance.

\begin{tabular}{|c|c|c|c|c|c|}
\hline $\begin{array}{l}\text { Bonding type } \\
\text { and binding } \\
\text { energy }\end{array}$ & Morphology and structure & Pore size & $\begin{array}{l}\text { Surface } \\
\text { area } \\
\left(\mathrm{m}^{2} / \mathrm{g}\right)\end{array}$ & $\begin{array}{l}\text { Capacity after } \\
\quad(x) \text { cycles }\end{array}$ & Reference \\
\hline \multirow{5}{*}{$(1.64-2.84 \mathrm{eV})$} & $\begin{array}{l}\text { SDCNTs: S incorporated into } \\
\text { voids/defects in disordered carbon } \\
\text { nanotubes }\end{array}$ & $2-4 \mathrm{~nm}$ & - & $672(100)$ & {$[14 c]$} \\
\hline & $\begin{array}{l}\text { C/S-50-T: sulfur dispersed in the } \\
\text { micro-mesoporous carbon spheres }\end{array}$ & $1.7-6 \mathrm{~nm}$ & 1650 & $860(500)$ & [93] \\
\hline & $\begin{array}{l}\text { 3D porous carbon containing high } \\
\text { sulfur nanoparticles }\end{array}$ & $\begin{array}{c}1.5-25 \\
\mathrm{~nm}\end{array}$ & 628.5 & 917 (200) & [94] \\
\hline & $\begin{array}{l}\text { LBL-S: layer-by-layer nano- } \\
\text { assembled sulfur electrode }\end{array}$ & - & - & $630(600)$ & [74] \\
\hline & $\begin{array}{l}\text { GO-S: sulfur-doped on the highly } \\
\text { developed porous layered GO }\end{array}$ & - & $1.66^{*}$ & $954(50)$ & {$[47 \mathrm{~b}]$} \\
\hline \multirow{5}{*}{$\begin{array}{c}\text { O-S } \\
(0.84 \sim 1.95 \mathrm{eV})\end{array}$} & $\begin{array}{l}\text { HC-2 }(9 \% \text { O): hierarchical porous } \\
\text { carbon }\end{array}$ & $\begin{array}{l}6 \mathrm{~nm}, \\
600-700\end{array}$ & $300-400$ & $750(60)$ & [95] \\
\hline & $\begin{array}{l}\mathrm{C} / \mathrm{S} \text { : porous spheres consist of } \\
\text { aggregated short plates }\end{array}$ & - & - & $508(2000)$ & [96] \\
\hline & $\begin{array}{l}\text { MPNC-S: sulfur flowed into the } \\
\text { disordered mesoporous N-doped } \\
\text { carbon }\end{array}$ & $10-20 \mathrm{~nm}$ & 824.3 & $800(100)$ & [79] \\
\hline & $\begin{array}{l}\text { SCNT-300: sulfur impregnated into } \\
\text { oxygen-functionalized CNTs }\end{array}$ & - & - & $800(600)$ & [76] \\
\hline & $\begin{array}{l}\text { S/C: sulfur into the grape-like } \\
\text { structured microporous carbon } \\
\text { spheres grown on CNTs }\end{array}$ & $1 \mathrm{~nm}$ & 915 & $720(100)$ & {$[40]$} \\
\hline \multirow{5}{*}{$\begin{array}{c}\text { N...Li } \mathrm{Li}_{2} \mathrm{~S}_{\mathrm{x}} \\
(0.25-4.08 \mathrm{eV})\end{array}$} & $\begin{array}{l}\text { G-NDHCS-S: sulfur impregnated } \\
\text { into the N-doped hollow carbon, } \\
\text { which was further wrapped by } \\
\text { graphene }\end{array}$ & $5-10 \mathrm{~nm}$ & 807.1 & $936(100)$ & [81] \\
\hline & $\begin{array}{l}\text { S@NG: ultrafine sulfur wrapped in } \\
\text { N-doped porous graphene } \\
\text { nanosheets }\end{array}$ & $3-30 \mathrm{~nm}$ & 643.5 & $752(300)$ & [89] \\
\hline & $\begin{array}{l}\mathrm{g}-\mathrm{C}_{3} \mathrm{~N}_{4} / \mathrm{S} \text { : sulfur composited with } \\
\text { nanoporous graphitic carbon nitride }\end{array}$ & $2-10 \mathrm{~nm}$ & 615 & $620(500)$ & {$[46]$} \\
\hline & $\begin{array}{l}\text { NCNT-S: sulfur infiltrated into the } \\
\text { open-structured interspace of N- } \\
\text { doped CNTs }\end{array}$ & $10-13 \mathrm{~nm}$ & 233 & $645(200)$ & [97] \\
\hline & $\begin{array}{l}\text { MNCS/CNT-S: sulfur confined in } \\
\text { CNT-interpenetrated mesoporous N- }\end{array}$ & $20-40 \mathrm{~nm}$ & 615.02 & $1200(200)$ & {$[80]$} \\
\hline
\end{tabular}




\begin{tabular}{|c|c|c|c|c|c|}
\hline & \multicolumn{5}{|l|}{ doped carbon spheres } \\
\hline & $\begin{array}{l}\text { NMHC-2-S: sulfur confined well } \\
\text { within the porous carbon shell }\end{array}$ & $2-6 \mathrm{~nm}$ & 1462 & $980(100)$ & [98] \\
\hline & $\begin{array}{l}\text { S@Co-N-GC: polyhedron-like } \\
\text { morphology with microporous } \\
\text { structure }\end{array}$ & $2-3 \mathrm{~nm}$ & 308.89 & $621(500)$ & [78] \\
\hline \multirow{3}{*}{$\begin{array}{c}\text { P...Li } \mathrm{Li}_{2} \mathrm{~S}_{\mathrm{x}} \\
(0.94-1.39 \mathrm{eV} \\
\text { Li2S8 1.86) }\end{array}$} & $\begin{array}{l}\text { PCNT@S: sulfur flowed into the } \\
\text { porous P-doped CNTs }\end{array}$ & $5-10 \mathrm{~nm}$ & 132 & 917 (100) & [99] \\
\hline & $\begin{array}{l}\text { p-NP-G: porous N,P dual doped } \\
\text { graphene }\end{array}$ & $1-5 \mathrm{~nm}$ & 573.7 & $638(500)$ & [100] \\
\hline & $\begin{array}{l}\text { Conductive molecular framework } \\
\text { derived } \mathrm{Li}_{2} \mathrm{~S} / \mathrm{N}, \mathrm{P} \text {-codoped carbon }\end{array}$ & $1-5 \mathrm{~nm}$ & 695 & $500(300)$ & [101] \\
\hline \multirow{2}{*}{$\begin{array}{c}\text { B... } \operatorname{Li}_{2} S_{x} \\
(0.9-5.15 \mathrm{eV})\end{array}$} & $\begin{array}{l}\text { BMC/S:B-doped carbon coated on } \\
\text { CNTs with outmost sulfur layer }\end{array}$ & $0.34 \mathrm{~nm}$ & 461.73 & $562(500)$ & [102] \\
\hline & $\begin{array}{l}\text { G-NBCL/S: sulfur distributed in } \\
\text { graphene-supported N, B rich } \\
\text { carbon layer }\end{array}$ & $1-10 \mathrm{~nm}$ & 91.0 & $556(500)$ & [103] \\
\hline \multirow{3}{*}{$\begin{array}{c}\text { Mn-S } \\
(1.84-2.59 \mathrm{eV})\end{array}$} & $\begin{array}{l}\text { Core-shell structured } \gamma-\mathrm{MnO}_{2} \text { coated } \\
\text { sulfur }\end{array}$ & - & - & $802(300)$ & [52] \\
\hline & $\begin{array}{l}\text { Homogeneous sulfur coating on the } \\
\mathrm{MnO}_{2} \text { nanosheets }\end{array}$ & - & - & $1030(200)$ & [53] \\
\hline & $\begin{array}{l}\text { NS-Core/ } \mathrm{MnO}_{2} \text { : sulfur nanoparticles } \\
\text { coated with } \mathrm{MnO}_{2} \text { shell }\end{array}$ & - & - & $950(300)$ & [29b] \\
\hline \multirow{3}{*}{$\begin{array}{l}\mathrm{V} \ldots \mathrm{Li}_{2} \mathbf{S}_{\mathrm{x}} \\
(\sim 1.97 \mathrm{eV})\end{array}$} & $\begin{array}{l}\text { S/VCM: sulfur infiltrated into the } \\
\mathrm{V}_{2} \mathrm{O}_{3} \text { nanoparticles modified carbon } \\
\text { microspheres }\end{array}$ & $1-10 \mathrm{~nm}$ & 710 & $921(100)$ & [57] \\
\hline & $\begin{array}{l}\mathrm{V}_{2} \mathrm{O}_{5} / \mathrm{C} \text { additive: } \mathrm{V}_{2} \mathrm{O}_{5} \text { xerogel } \\
\text { particles distributed on the surface } \\
\text { of spherical carbon black }\end{array}$ & - & - & 〜900 (100) & [104] \\
\hline & $\begin{array}{l}\text { VCNF film: } \mathrm{V}_{2} \mathrm{O}_{5} \text {-decorated carbon } \\
\text { nanofiber interlayer: }\end{array}$ & - & - & $576(1000)$ & [105] \\
\hline \multirow{3}{*}{$\begin{array}{c}\text { Cu-S } \\
(\sim 1.56 \mathrm{eV})\end{array}$} & $\begin{array}{l}\text { CAPC-S: } \mathrm{Cu} \text { anchored cross-linked } \\
\text { polymer-coated sulfur electrode }\end{array}$ & - & - & $647(100)$ & [71] \\
\hline & $\begin{array}{l}\text { MC-Cu-S: Cu nanoparticles } \\
\text { percolate through the microporous } \\
\text { carbon to anchor sulfur }\end{array}$ & $2-5 \mathrm{~nm}$ & 1650 & $630(500)$ & [42] \\
\hline & $\begin{array}{l}\text { Sulfur infiltrated into the micro- } \\
\text { mesoporous carbon aerogel } \\
\text { modified by } \mathrm{CuS} \text { quantum dot }\end{array}$ & $4-8 \mathrm{~nm}$ & 726 & $1073(100)$ & [106] \\
\hline
\end{tabular}




\begin{tabular}{|c|c|c|c|c|c|}
\hline & $\begin{array}{l}\text { Sulfur impregnated in porous carbon } \\
\text { with } \mathrm{Cu} \text { additive }\end{array}$ & - & - & $1300(80)$ & [107] \\
\hline \multirow{3}{*}{$\begin{array}{c}\text { Ti-S } \\
(\sim 2.33 \mathrm{eV})\end{array}$} & $\begin{array}{l}\mathrm{SCM} / \mathrm{S}-\alpha-\mathrm{TiO}_{2}: \text { sulfur impregnated } \\
\text { mesoporous carbon with } \mathrm{TiO}_{2} \\
\text { additive }\end{array}$ & $5 \mathrm{~nm}$ & $\begin{array}{c}275 \text { for } \\
\mathrm{TiO}_{2}\end{array}$ & $800(200)$ & [108] \\
\hline & Yolk-shell nanoarchitecture $\mathrm{S}-\mathrm{TiO}_{2}$ & $3 \mathrm{~nm}$ & - & $690(1000)$ & [109] \\
\hline & $\begin{array}{l}\mathrm{Ti}_{4} \mathrm{O}_{7} / \mathrm{S}: \mathrm{Ti}_{4} \mathrm{O}_{7} \text { crystals loosely } \\
\text { bound with interspersed carbon with } \\
\text { sulfur distributed in the micro- } \\
\text { mesoporous }\end{array}$ & $2-10 \mathrm{~nm}$ & 290 & $\sim 800(250)$ & {$[110]$} \\
\hline \multirow{3}{*}{$\begin{array}{c}\text { Co-S } \\
(3.79 \mathrm{eV})\end{array}$} & $\begin{array}{l}\mathrm{CoS}_{2} / \mathrm{CP} \text { interlayer: porous } \mathrm{CoS}_{2} \\
\text { nanosheets array with uniform } \\
\text { thickness grown on the carbon paper }\end{array}$ & - & - & $817(200)$ & [111] \\
\hline & $\begin{array}{l}\mathrm{CoS}_{2} \text { incorporated into } \\
\text { graphene/sulfur cathodes }\end{array}$ & - & 709 & $\sim 750(250)$ & [112] \\
\hline & $\begin{array}{l}\mathrm{Co}_{3} \mathrm{O}_{4}-\mathrm{S}: \text { sulfur encapsulated by } \\
\text { ultrathin } \mathrm{Co}_{3} \mathrm{O}_{4} \text { nanosheets }\end{array}$ & - & 80.35 & $656(200)$ & [113] \\
\hline \multirow{2}{*}{$\begin{array}{c}\text { Fe-S } \\
(0.87-1.85 \mathrm{eV})\end{array}$} & $\begin{array}{l}\text { Pyrite } \mathrm{FeS}_{2} \text { as an additive in the } \\
\text { sulfur-carbon black electrodes }\end{array}$ & - & - & $700(200)$ & [114] \\
\hline & $\begin{array}{l}\text { Porous } \mathrm{Fe}_{2} \mathrm{O}_{3} \text { microcubes } \\
\text { composited with sulfur }\end{array}$ & $1-10 \mathrm{~nm}$ & - & $575(100)$ & [115] \\
\hline \multirow{3}{*}{$\begin{array}{c}\text { Mo-S } \\
(\sim 3.97 \mathrm{eV})\end{array}$} & $\begin{array}{l}\text { Flexible } \mathrm{MoS}_{2} \text { flakes encapsulated } \\
\text { sulfur nanoparticles }\end{array}$ & - & - & $956(300)$ & [116] \\
\hline & $\begin{array}{l}\text { Mo-S-G: sulfur uniform distributed } \\
\text { in the porous graphene with Mo } \\
\text { nanoparticles decoration }\end{array}$ & - & - & $\sim 800(100)$ & {$[117]$} \\
\hline & $\begin{array}{l}\text { MolyS50: sulfur intimate mixed } \\
\text { with thin } \mathrm{MoS}_{2} \text { sheets }\end{array}$ & - & - & $\sim 850(500)$ & [118] \\
\hline \multirow{3}{*}{$\begin{array}{c}\mathrm{Ni}-\mathrm{S} \\
(\mathbf{0 . 7 2 - 2 . 5 9} \mathrm{eV})\end{array}$} & $\begin{array}{l}\text { S/NiS@C-HS: sulfur } \\
\text { homogeneously distributed in the } \\
\text { 3D carbon hollow spheres doped by } \\
\text { nanosized NiS }\end{array}$ & - & 241 & $717(200)$ & [119] \\
\hline & $\begin{array}{l}\mathrm{S} @ \mathrm{a}-\mathrm{NiS}_{2}: \text { nanocrystal S particles } \\
\text { embedded in the amorphous } \mathrm{NiS}_{2} \\
\text { host material }\end{array}$ & - & - & $954(1200)$ & {$[120]$} \\
\hline & $\begin{array}{l}\text { Sulfur injected into the } \mathrm{Ni} \\
\text { foam @ carbon-shell with a pie-like } \\
\text { architecture }\end{array}$ & - & - & $669(100)$ & [121] \\
\hline
\end{tabular}




\subsection{Lithium metal anode}

As we discussed above, a long-term favorite has been lithium metal batteries due to their high theoretical energy density. By replacing the standard graphite electrode with a solid lithium electrode, the size and weight of the battery are significantly reduced. Although the benefits of lithium metal batteries, the critical failure mechanism is the primary dendrite growth at the lithium surface during the charging process and finally results in many serious problems (Figure 3). Therefore, it is critical to have a good understanding of the mechanism of Li dendrite formation and growth to mitigate further eliminate Li dendrites.

2.3.1 Mechanism and modeling of Li dendrite growth.

Lithium morphology and failure mechanisms. The deposition of Li could form several types of surface morphology: including moss-like, particulate, or dendritic (needle-like) deposits. The dendritic aggregation is the most detrimental to the cycling efficiency and battery safety since dendrites could accelerate the capacity fade due to the formation of electrically isolated Li (dead "Li"), or even trigger the internal short when piercing through the battery separator. Yoshimatsu et al. measured the lithium electrode surface morphologies during cycling and observed both particulate and needle-like Li (Figure 11a) on the Li electrode after cycling. ${ }^{[122]}$ However, the needle-like Li tended to become "dead Li" during stripping, as schemed in Figure 11b. Aurbach and co-workers observed the failure mechanism of Li metal by AFM, as schematically presented in Figure 11c. ${ }^{[123]}$ Because the surface films formed on lithium in most of the relevant and commonly used electrolyte solutions are comprised of Li salts, their cohesion and flexibility are very limited. Hence, as seen in Figure 11c, these surface films cannot properly accommodate the morphological changes of the Li metal upon Li deposition and dissolution (due to the nonuniformity of these processes). The surface films formed on lithium can be easily cracked, and 
the passivation is broken during both $\mathrm{Li}$ deposition and dissolution. This leads to dendrite formation and a massive loss of both lithium and solution species due to the surface reactions and the 'repair' of the surface films (on an increasing Li surface area).
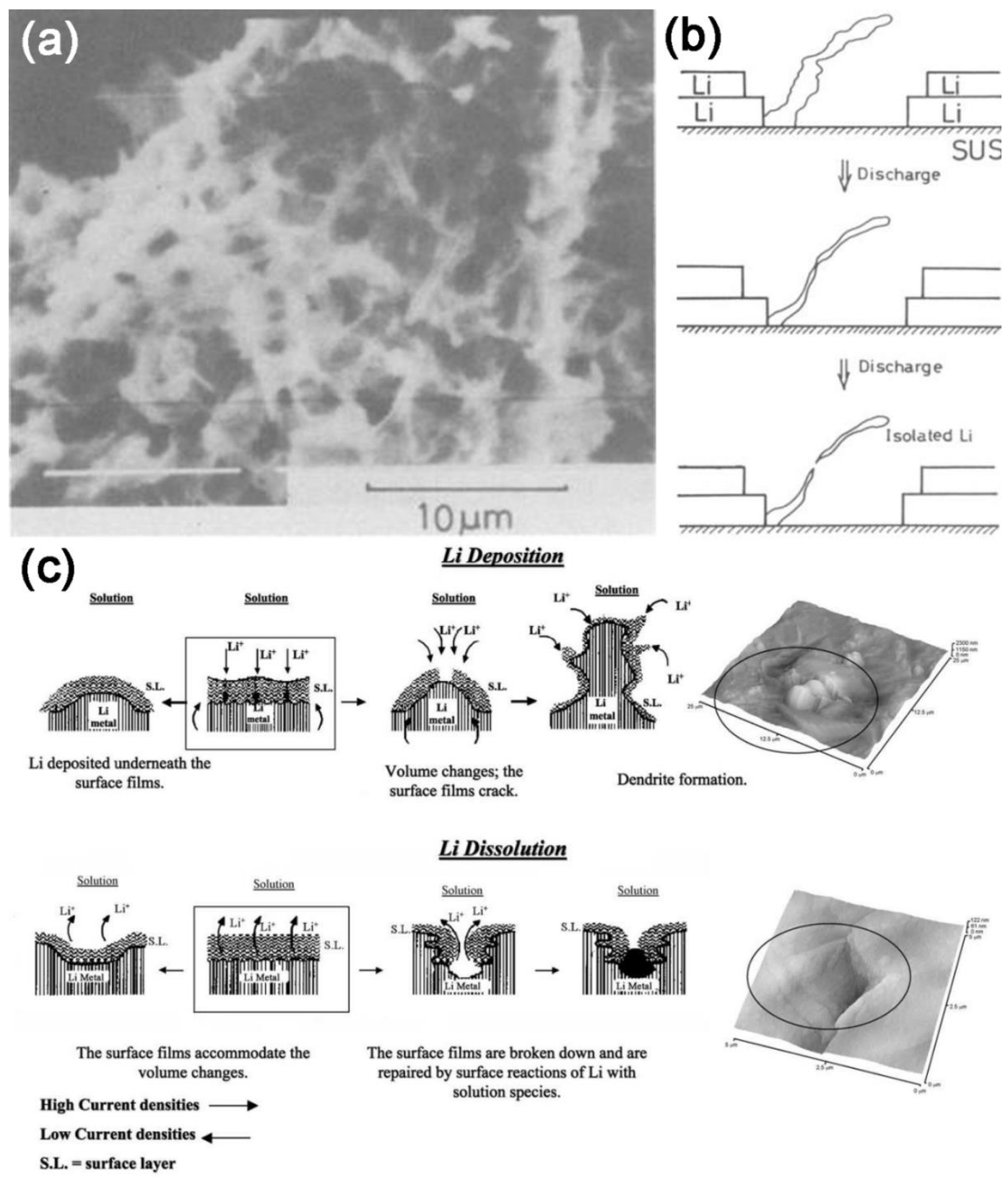

Figure 11 (a) SEM image of the Li surface of the Li cell cycled to the end of its life. (b) Schematic diagrams showing the separation of needle-like Li. (c) A description of the morphology and failure mechanisms of lithium electrodes and relevant AFM images. 
Dendritic growth models in lithium/polymer cells. Brissot and Chazalviel et al. described the dendritic growth mechanism based on the calculation of the concentration gradient in $\mathrm{Li}$ symmetrical lithium/polymer cells under galvanostatic conditions. ${ }^{[124]}$ Start from the evolution of ionic concentrations $\mathrm{C}_{\mathrm{a}}$ and $\mathrm{C}_{\mathrm{c}}$ and supposing the constant diffusion coefficients and mobilities, they obtained concentration profile using the following equation:

$$
\frac{\partial C}{\partial x}(\mathbf{x}=\mathbf{0})=\frac{-J}{e D\left(1+\frac{\mu_{c}}{\mu_{a}}\right)}
$$

where $J$ is the effective electrode current density, $D$ is the ambipolar diffusion coefficient, $e$ is the electronic charge, $\mu_{\mathrm{a}}$ and $\mu_{\mathrm{Li}+}$ are the anionic and $\mathrm{Li}^{+}$mobilities. From Eqn. 5, two different conditions for a symmetric cell can be anticipated, with the inter-electrode distance $L$ and the initial Li salt concentration $C_{0}$. (a) If $d C / d x<2 C_{0} / L$, the ionic concentration evolves to a steady state where the concentration gradient is constant and the cell potential attains a stationary value (Figure 12a); (b) If $d C / d x>2 C_{0} / L$, the ionic concentration goes to zero at the negative electrode at a time called "Sand's time" $\tau$ (Figure $12 \mathrm{~b}$ ), which varies as,

$$
\begin{gathered}
\tau=\pi \mathrm{D}\left(\frac{e C_{0}}{2 J t_{a}}\right)^{2} \\
\mathrm{t}_{a} \cong \mathbf{1}-t_{L i^{+}}=\frac{\mu_{a}}{\mu_{a}+\mu_{L i^{+}}}
\end{gathered}
$$

where $\mathrm{t}_{\mathrm{a}}$ and $\mathrm{t}_{\mathrm{Li}}$ represent the anionic and $\mathrm{Li}^{+}$transference number, respectively. Brissot and Chazalviel et al. indicated that the anionic and $\mathrm{Li}^{+}$concentrations exhibit different behaviors at the Sand's time, leading to an excess of positive charge at the negative electrode. This behavior will result in a local space charge, very close to the Sand's time.

Newman's group also provided lots of models for dendrite growth in Li/polymer systems. ${ }^{[125]}$ A one-dimensional model was proposed to investigate the dendrite growth mechanism, as 
schemed in Figure 12c. Start from the salt concentration profile based on the Fick's second law as the following equation:

$$
\frac{\partial C}{\partial t}=\mathrm{D} \frac{\partial^{2} C}{\partial^{2} y}
$$

where $D$ is the salt diffusion coefficient and $t$ is time. The equation is subject to the boundary conditions:

$$
\begin{gathered}
\mathrm{i}=-\left.\frac{D F}{\left(1-t_{+}^{0}\right)} \frac{\partial C}{\partial y}\right|_{y=0} \\
\frac{1}{L} \int_{0}^{L} C(y, t) d y=C_{b} \\
\mathrm{C}=C_{b}, \text { at } \mathrm{t}=0
\end{gathered}
$$

where $F$ is Faraday's constant and $t_{+}^{0}$ is the cation transference number. After being nondimensionalized and solved the equations using separation of variables, the final solution of the concentration profile could be expressed as:

$$
\theta=-\xi+\frac{1}{2}-4 \sum_{k=1}^{\infty} \frac{1}{(2 k-1)^{2} \pi^{2}} e^{-(2 k-1)^{2} \pi^{2} \tau} \times \cos [(2 k-1) \pi \xi] \quad \text { Eqn. } 12
$$

where $\xi, \theta$, and $\tau$ could be expressed as:

$$
\begin{gathered}
\xi=\frac{y}{L} \\
\tau=\frac{D t}{L^{2}} \\
\boldsymbol{\theta}=\frac{\left(C-C_{b}\right) D F}{\left(1-t_{+}^{0}\right) i L}
\end{gathered}
$$

The final concentration profiles based on this equation are plotted in Figure 12d with the values of $5 \times 10^{-12} \mathrm{~m}^{2} / \mathrm{s}$ for $D$ and 0.3 for $t_{+}^{0}$. The interelectrode spacing $(L)$ was set to $100 \mu \mathrm{m}$. It can be noted that under these conditions it took about $2000 \mathrm{~s}$ for the concentration profile to reach a steady state. 
They further calculated the potential profiles using the results of the section on the concentration profile. By assuming the concentration of the salt-affected only the cathodic rate, a Butler-Volmer type expression used for the reaction kinetics could be described as:

$$
\frac{i}{F}=k_{a} \exp \left(\frac{\alpha_{a} F}{R T} \varphi\right)-k_{c} \operatorname{Cexp}\left(\frac{-\alpha_{c} F}{R T} \varphi\right)
$$

Where $k_{a}$ and $k_{c}$ are anodic and cathodic rate constants, $R$ is the ideal gas constant, and $T$ is the absolute temperature of the cell. The $\alpha_{\mathrm{i}}$ are transfer coefficients. With the overpotential as boundary conditions, the instantaneous potential $(\Phi)$ at any point across the cell was established:

$$
\Phi(\mathbf{y}, \mathbf{t})=\eta_{s}-\frac{i}{A} \int_{0}^{y} \frac{1}{C} d y+\frac{R T}{F}\left(1-2 t_{+}^{0}\right) \ln \left[\frac{C(y, t)}{C(0, t)}\right]
$$

Where $\eta_{s}$ is the surface overpotential, $\Lambda$ is the equivalent conductance of the electrolyte and can be expressed using the Nernst-Einstein relation:

$$
\Lambda=\frac{F^{2} D}{2 t_{+}^{0}\left(1-t_{+}^{0}\right) R T}
$$

Based on these equations above, Newman et al. obtained the potential distribution in the cell, as shown in Figure 12e. With the concentration and potential profiles, they proposed a dendrite growth model. By assuming that mass transfer and surface forces dominate the kinetics of dendrite growth, they added well-defined thermodynamic reference points to the analysis, as diagramed in Figure 12f. In the drawing, phase $\alpha$ is a reference electrode of a given kind, placed in the vicinity of the dendrite tip. Implicitly, they also assumed that there was only one dendrite and was small enough that will not greatly affect the concentration and potential profiles in the cell. Finally, they connected the dendrite tip growth velocity $\left(v_{\text {tip }}\right)$ with the concentration and overpotential profiles in Eqn. 19 with a typical growth profile for lithium dendrite in Figure 12g.

$$
\begin{gathered}
v_{t i p}=\frac{i_{n}(C, \eta) V}{F} \\
\frac{\partial y_{t i p}}{\partial t}=\frac{V}{F} i_{n}(C, \eta)=\frac{V}{F} i_{n}\left(y_{t i p}, t\right)
\end{gathered}
$$



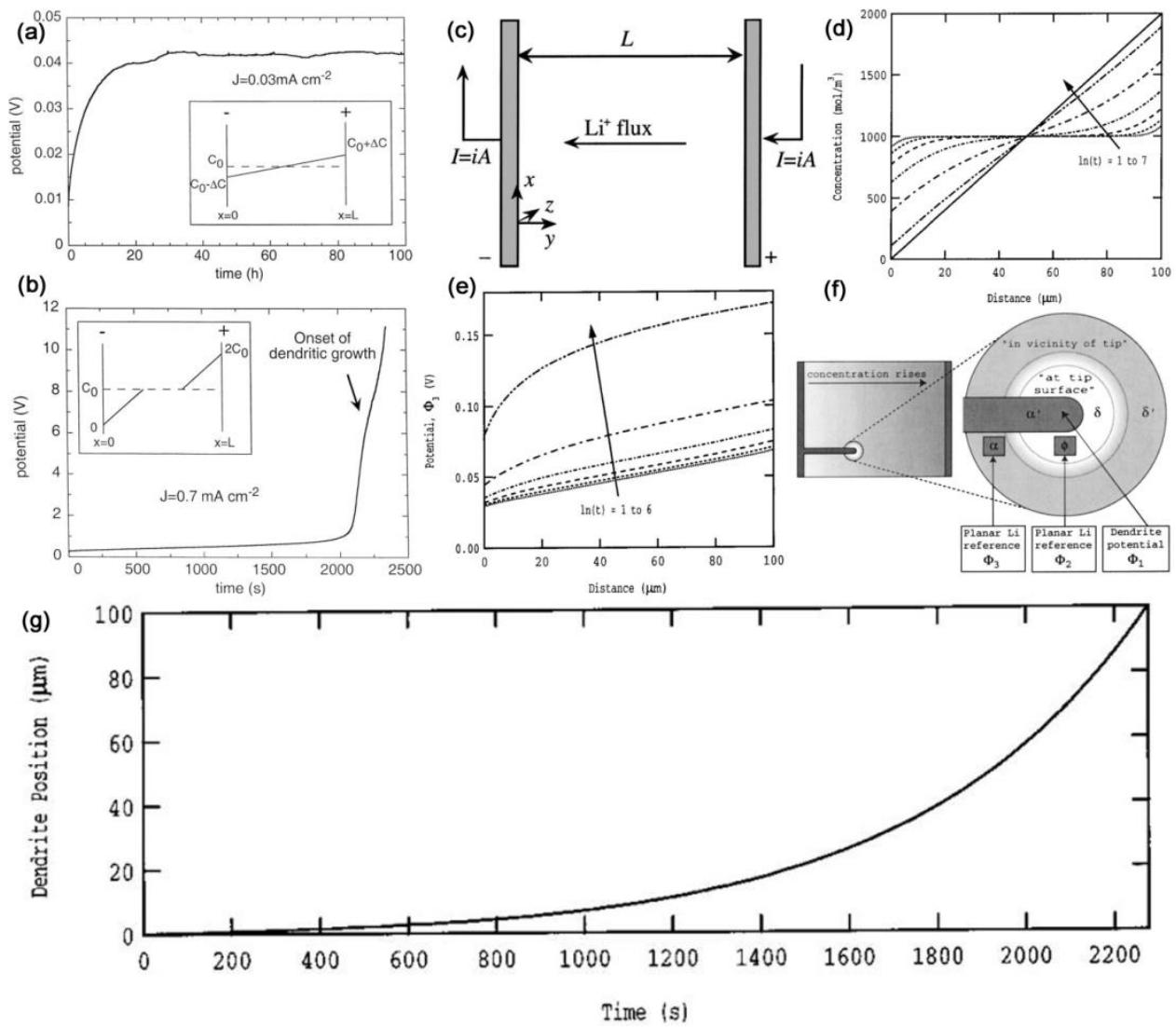

Figure 12 For a given distance $\mathrm{L}$ between the electrodes, (a) if $J<J^{*}$, the system evolves to a steady state where the concentration varies linearly from $\mathrm{C}_{0}-\Delta \mathrm{C}$ at the negative electrode to $\mathrm{C}_{0}+\Delta \mathrm{C}$ at the positive electrode, (b) if $J<J^{*}$ (semi-infinite approximation), the ionic concentration drops to zero and the cell potential diverges at the Sand's time. (c) Schematic diagram of the galvanostatic polarization cell. (d) Concentration and (e) potential profiles in the galvanostatic cell. (f) Diagram of the region near the dendrite tip. (g) Typical growth profile for a lithium dendrite.

\subsubsection{Li dendrite prevention}

In the previous research, considerable efforts have been directed towards the challenges of $\mathrm{Li}$ metal anode for the practical application. Basically, these methods can be summarized into three aspects, e.g., (1) modifying the organic electrolytes, ${ }^{[126]}$ (2) building three-dimensional host 
materials for the Li metal, ${ }^{[127]}$ and (3) improving the interface between the Li metal anode and electrolyte. $^{[128]}$

Modifying the organic electrolytes. This method is actually to in situ forms an SEI film on the Li metal surface. Due to the unstable interface between the reactive $\mathrm{Li}$ metal and the organic electrolyte, SEI film forms immediately on the Li surface. Unstable SEI film increases the potential of Li dendrite penetration with newly exposed Li metal to the organic electrolyte. The repeated SEI formation consumes both $\mathrm{Li}$ and electrolyte results in the continued increase in the cell resistance and poor Coulombic efficiency of the battery. However, the quality SEI with high Li ionic conductivity, dense film, small thickness, and high elastic strength can effectively suppress the breakthrough by Li dendrites. Therefore, stable SEI films are expected to be obtained by modifying the components of organic electrolytes. Gofer et al. reported that adding EC or PC to the DOL electrolytes can improve the uniformity of the SEI and the homogeneous Li deposition and stripping. It will also increase the Coulombic efficiency of the process. ${ }^{[129]}$ However, this long cycle life was gained at a slow charge rate $(\mathrm{ca} . \mathrm{C} / 13)$ and half depth of charge/discharge. Quick charging and deep cycling will still lead to Li dendrite growth and short circuit of the cells. Naoi and co-workers reported a salt, Li bis(per-fuoroethylsulfonylimide) ( $\left.\mathrm{LiN}\left(\mathrm{C}_{2} \mathrm{~F}_{5} \mathrm{SO}_{2}\right), \mathrm{LiBETI}\right)$, which can form a very stable, thin, uniform, and a compact surface film containing mainly the LiF component and consequently give a slightly higher cycling efficiency than $\mathrm{LiPF}_{6}$, LiTFSI, or $\mathrm{LiCF}_{3} \mathrm{SO}_{3}$ in EC/DME solvents. ${ }^{[130]}$ Other functional additives such as $\mathrm{CO}_{2} / \mathrm{SO}_{2}$, hydrogen fluoride ( $\mathrm{HF}$ ), and vinylene carbonate (VC) are also employed to enhance the SEI films on the Li surface.

Building 3D host materials for Li metal. Li deposition/dissolution is an inherent process at the interface of Li metal/electrolyte during normal cycling. Therefore, the morphology control of the 
Li deposited surface is a key to the successful development of Li metal batteries. The current collector affects the nucleation at the initial period of Li plating, which is decisive for the morphology of the subsequently plated Li. Most of the current collectors used in the $\mathrm{Li}$ batteries are planar, such as conventional $\mathrm{Cu}$ and $\mathrm{Li}$ foils. The initial plating of $\mathrm{Li}$ on the planar current collector is prone to inhomogeneous $\mathrm{Li}$ particle deposition, followed by the growth of $\mathrm{Li}$ dendrites on the Li particles. The use of 3D structures is proposed as an ideal approach to address this problem. Liang et al. utilized oxidized polyacrylonitrile (PAN) nanofibers on top of current collectors to modify the Li metal anode and form flat surfaces. ${ }^{[131]}$ Because of the insulating nature of PAN, the 3D structure introduced in this method can only work as a scaffold to guide the growth of the Li metal. Besides insulating networks like PAN, Cheng et al. used a conductive graphene framework for $\mathrm{Li}$ deposition and successfully improved Coulombic efficiencies to around $97 \%$ for more than 100 cycles. ${ }^{[132]}$ Different from an insulating network, a conductive network can provide a large surface area for Li deposition so that areal current densities can be lessened significantly, which is beneficial for uniform current distribution. Yang et al. designed a 3D current collector with a submicron skeleton and high electroactive surface area, which significantly improved the electrochemical deposition behavior of Li (Figure 13a). Lithium particle is accommodated in the 3D structure without uncontrollable Li dendrites. With the growth of Li dendrites being effectively suppressed, the Li anode in the 3D current collector can run for $600 \mathrm{~h}$ without a short circuit and exhibits low voltage hysteresis. ${ }^{[127]} \mathrm{Ye}$ and co-workers indicated that the growth of $\mathrm{Li}$ dendrite can be mitigated by regulating the interfacial mass/charge transfer, and proposed a hybrid Li storage in onion-like, graphitized spherical C granules wired on a three-dimensional conducting skeleton (Figure 13b), which enhanced the negativity of surface charge of the $\mathrm{C}$ host to contribute to a uniform Li plating while also 
forming stable $\mathrm{Li} / \mathrm{C}$ intercalation compounds to offset any irreversible Li loss during cycling. As a result, the anode shows a suppressed dendrite formation and a high Li utilization $>95 \%$, enabling a practical Li-battery to strike a long lifespan of 1000 cycles at a surplus Li of merely $5 \% .{ }^{[133]}$

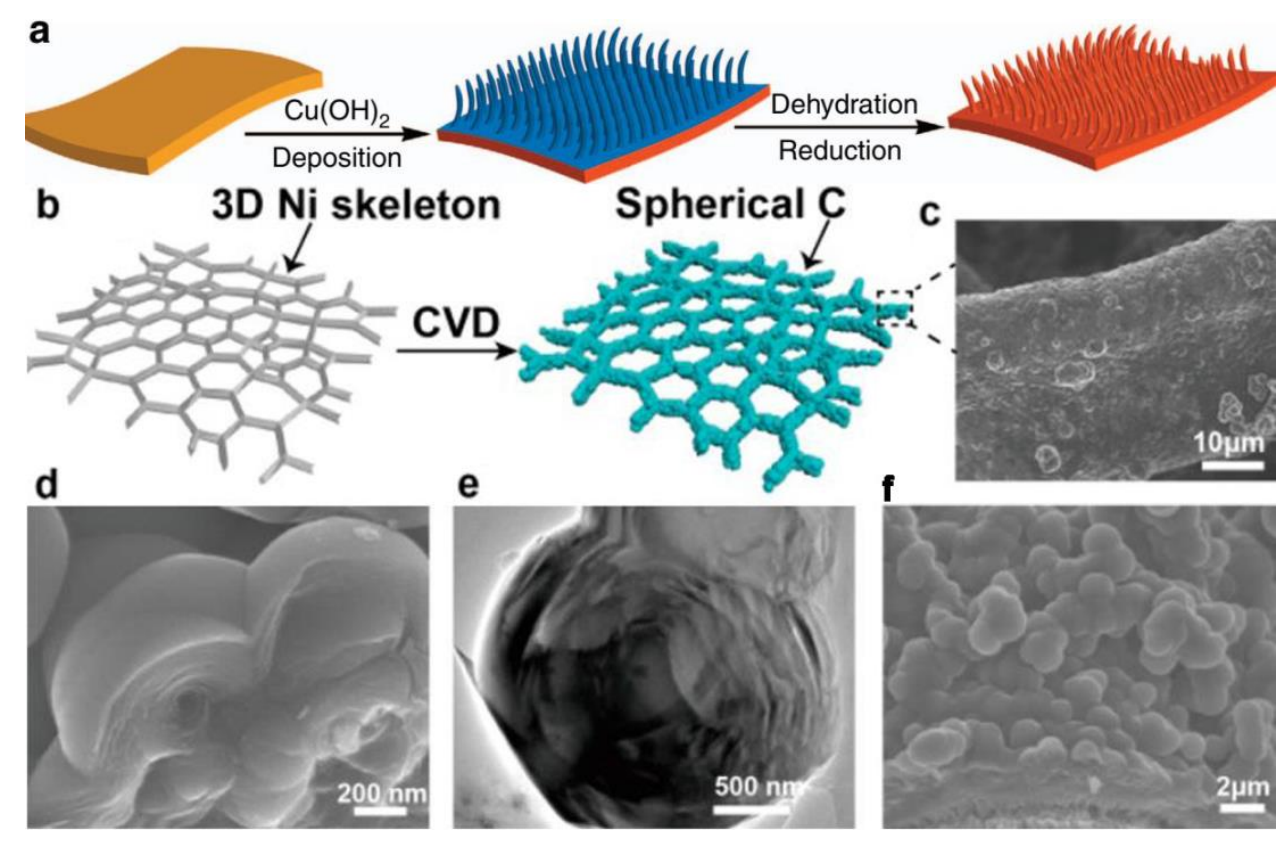

Figure 13 (a) Scheme of the procedures to prepare a 3D porous $\mathrm{Cu}$ foil from a planar $\mathrm{Cu}$ foil. Structural and morphological characterization results of the CMN. (b) Schematic illustration showing the preparation process of the CMN. (c) Low-magnification SEM image of the CMN. (d) Cross-sectional SEM image of spherical C and (e) sliced TEM image of a spherical C, which shows the onion-like structure. (f) Cross-sectional SEM image of the CMN.

Improving the interface between the Li metal anode and electrolyte. The improved interface between the Li metal anode and electrolyte can be achieved by two major methods. One is to cover the Li electrode with an ex situ-formed protective layer (or "artificial" SEI layer) by treating $\mathrm{Li}$ metal with chosen chemicals before its use in the battery electrolyte. Choi et al. coated the $\mathrm{Li}$ metal electrode with a cross-linked gel polymer electrolyte; ${ }^{[134]}$ Belov et al. 
protected the $\mathrm{Li}$ surface with polyacetylene via polymerization. ${ }^{[135]} \mathrm{Wu}$ et al. modified a $\mathrm{Li}$ electrode by exposing it to nitrogen to form a $\mathrm{Li}_{3} \mathrm{~N}$ layer. ${ }^{[136]}$ Zheng et al. reported that coating the lithium metal anode with a monolayer of interconnected amorphous hollow carbon nanospheres helps isolate the lithium metal depositions and facilitates the formation of stable solid electrolyte interphase (Figure 14a). ${ }^{[137]}$ They also showed that lithium dendrites do not form up to a practical current density of $1 \mathrm{~mA} \mathrm{~cm}^{-2}$. The Coulombic efficiency improves to $\sim 99 \%$ for more than 150 cycles. This is significantly better than the bare unmodified samples, which usually show rapid Coulombic efficiency decay in fewer than 100 cycles. The results indicated that nanoscale interfacial engineering could be a promising strategy to tackle the intrinsic problems of lithium metal anodes. Yang and co-workers reported a polymer/ceramic hybrid membrane that is Li-ion-conducting and electron insulating as a protective layer for the $\mathrm{Li}$ metal anode and demonstrate that it can remarkably enhance the stability of the anode. ${ }^{[138]}$ As shown in Figure $14 \mathrm{~h}$, the membrane consists of the garnet-type solid-state electrolyte $\mathrm{Li}_{7} \mathrm{La}_{3} \mathrm{Zr}_{1.75} \mathrm{Nb}_{0.25} \mathrm{O}_{12}$ (LLZNO) and a poly(ethyleneoxide) (PEO)-based polymer electrolyte. Because of the flexible nature of the polymer electrolyte, the hybrid membrane can easily accommodate large Li volume changes during Li plating and stripping. The ceramic LLZNO particles dispersed in the PEO electrolyte significantly enhance its mechanical properties, thereby suppressing the growth of the Li dendrites. In addition, the LLZNO particles decrease the PEO local crystallization, which is favorable for Li-ion transfer. Moreover, because the organic-inorganic hybrid membrane has a benign ionic conductivity and electronic resistivity, it allows a uniform Li-ion flux while inhibiting the electron flow. Therefore, the Li metal is deposited between the substrate and the protective layer, rather than on top of the coating layer, significantly reducing the possibility of dendrite formation. 

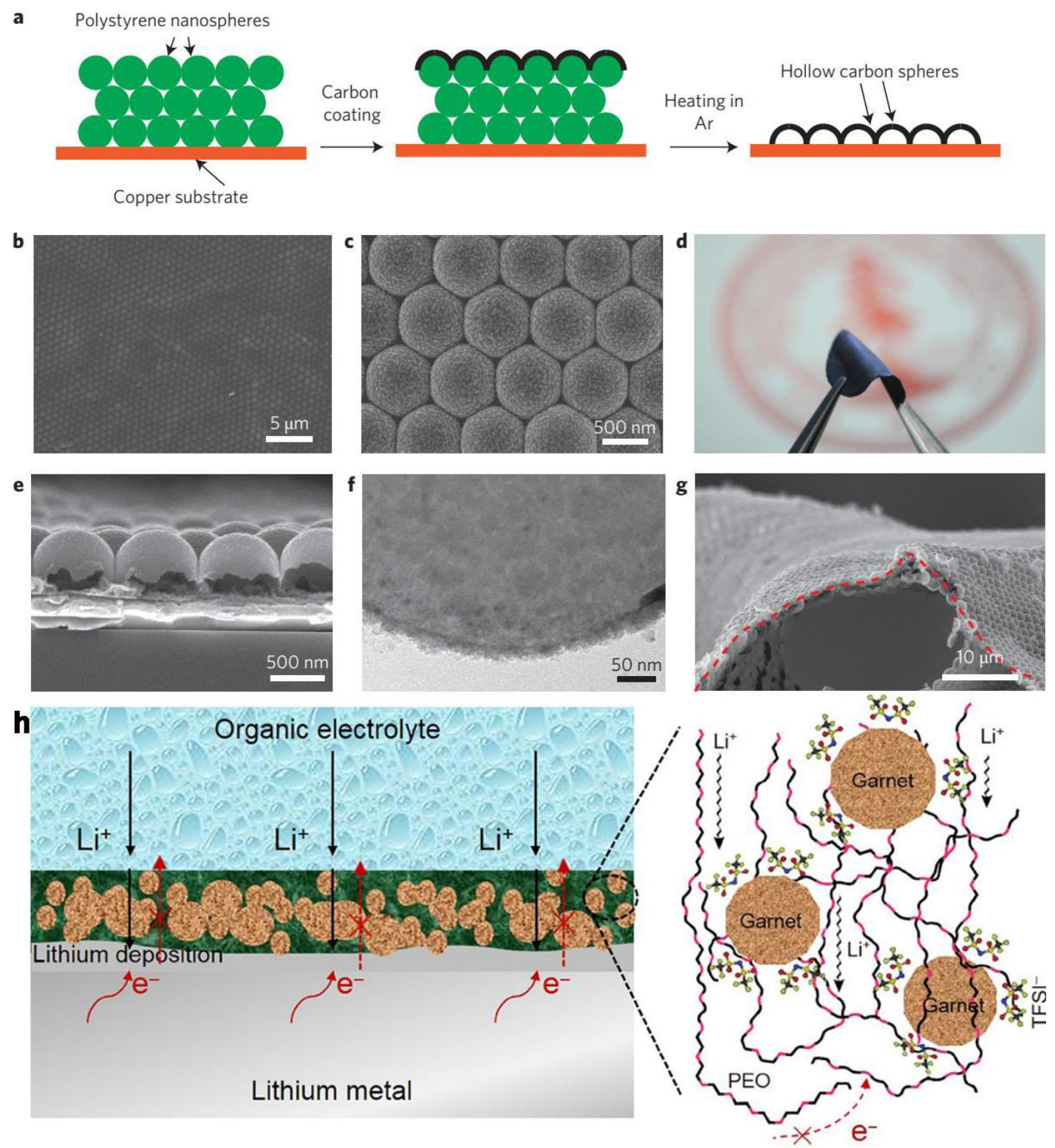

Figure 14 (a) Fabrication process for the hollow carbon nanosphere-modified $\mathrm{Cu}$ electrode. SEM images of the carbon-coated polystyrene nanoparticle array at low (b) and high (c) magnifications. (d) Digital camera image of the as-fabricated hollow carbon nanosphere thin film after removal of the polystyrene template. (e) Cross-sectional SEM image of the hollow carbon nanospheres. (f) TEM image of the hollow carbon nanospheres, with a wall thickness of $\sim 20 \mathrm{~nm}$. (g) SEM image of the hollow carbon nanosphere thin-film peeled off the $\mathrm{Cu}$ substrate. (h) Schematic of the PEO-garnet hybrid ion-conducting membrane coated on Li metal. 
The other way to improve the interface is to directly use solid-state electrolytes, such as polymer electrolytes, inorganic Li ion conductors, and the hybrid electrolytes. These electrolytes can avoid the unstable interface formation in the traditional organic liquid electrolytes. In addition, the much higher mechanical strength can suppress dendrite growth by mechanical blocking. Among the various $\mathrm{Li}^{+}$conductors, garnet-type $\mathrm{Li}_{7} \mathrm{La}_{3} \mathrm{Zr}_{2} \mathrm{O}_{12}$ (LLZO) exhibits high Liion conductivity, outstanding electrochemical stability with a wide operating voltage window, relatively good chemical stability, and robust mechanical strength. However, the poor interfacial contacts between garnet SSEs and the electrodes is one of the major challenges for the application of the garnet-based solid-state Li metal batteries.

Wang et al. reported a one-step soldering technique to quickly coat molten Li onto different substrates including metals, ceramics, and polymers (Figure 15). ${ }^{[139]}$ It is deduced that the surface energy and viscosity of the molten Li can be tuned by adding alloy elements, which improves the wettability against various substrates. When soldered onto the surface of garnetbased solid-state electrolytes (SSEs), the Li alloys exhibit significantly improved contact, which leads to an interface resistance as low as $\approx 7 \Omega \mathrm{cm}^{2}$. While cycling under high loads, the newly plated Li still maintains tight contact with the garnet surface and demonstrates excellent electrochemical stability. Several Li binary alloys as well as sodium ( $\mathrm{Na}$ ) binary alloys are successfully tested on various substrates to demonstrate the versatility of this soldering technique for potential battery applications. Tsai et al. also introduced modified interphase to increase the affinity between SSEs and $\mathrm{Li}^{\left[{ }^{[140]}\right.}$ By sputtering a thin Au buffer layer, the interface resistance could be dramatically reduced, which results in short-circuit-free cells when running a current density of $0.5 \mathrm{~mA} \mathrm{~cm}{ }^{-2}$ through the pellets. However, the short circuit still occurred at higher current density due to the inhomogeneous dissolution and deposition of metallic Li. 


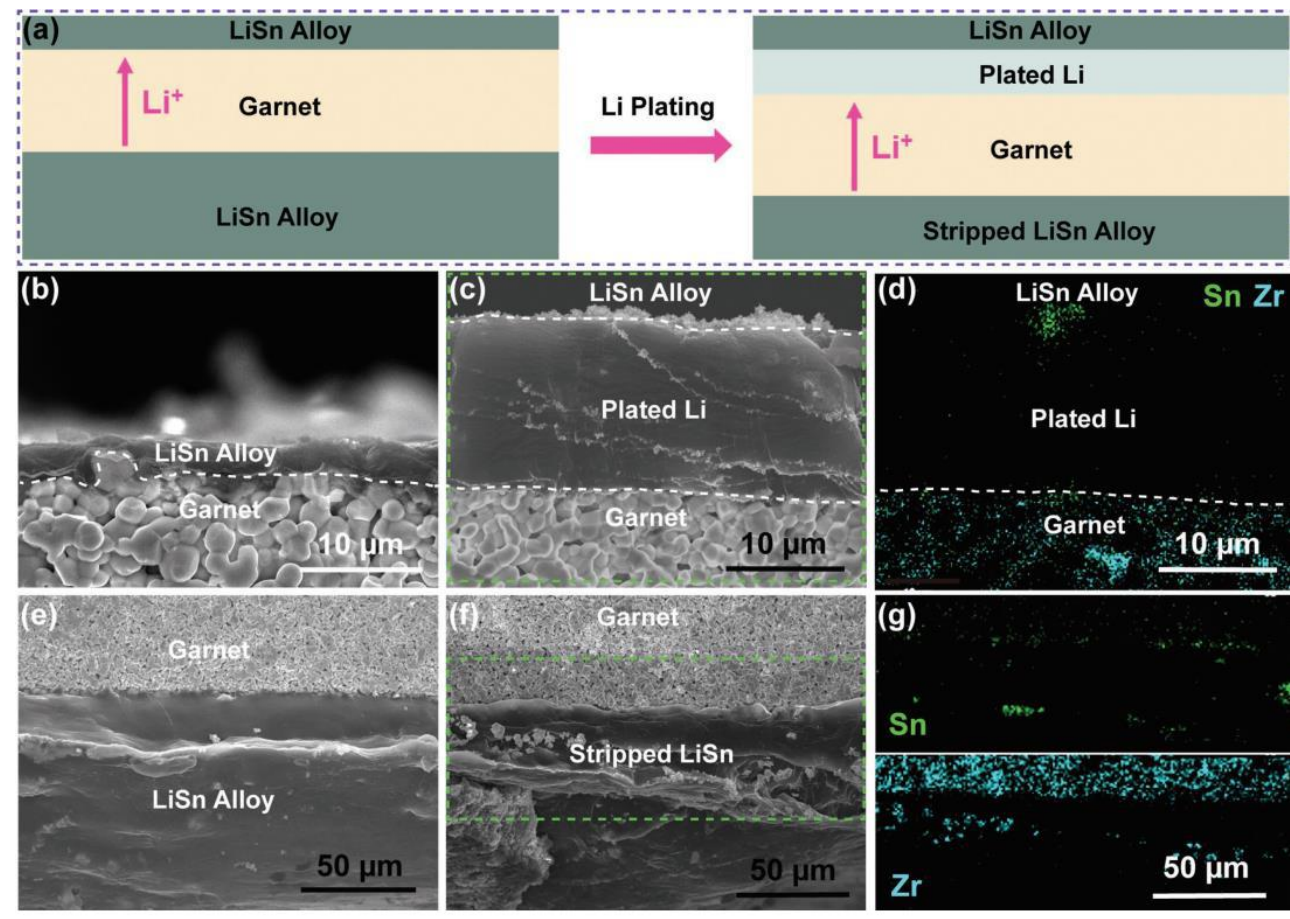

(h) Dendrite formation

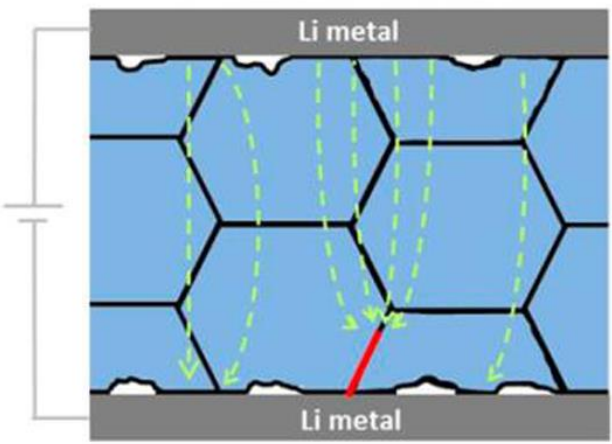

Inhomogeneous contact

$--\rightarrow \mathrm{Li}^{+}$current

Li dendrite

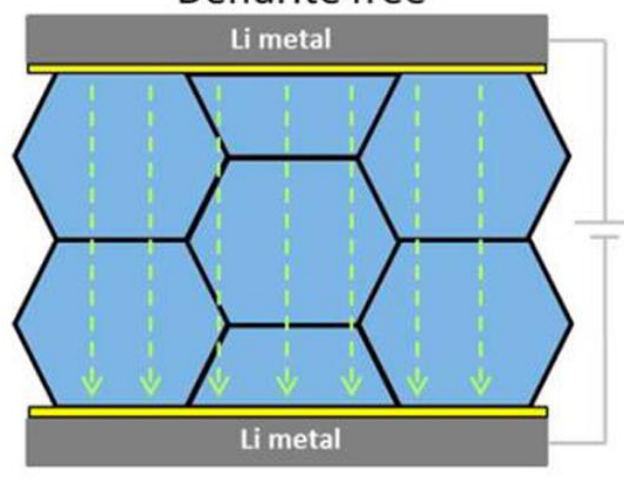

Homogeneous contact

Li alloyed buffer layer

Figure 15 (a) Schematic of the large capacity Li plating-stripping process for the Li$\mathrm{Sn} /$ garnet/Li-Sn symmetric cell. Cross-sectional SEM images of the Li-Sn alloy coated garnet before (b) and (c) after Li plating. (d) EDS mapping of the Li-plated area marked with a green dashed line in (c). Cross-sectional SEM images of the Li-Sn alloy coated garnet (e) before and (f) after Li stripping. (g) EDS mapping of the Li stripped area marked with a green dashed line in (f), where the leftover Sn does not show local accumulation at the interface. (h) Al-Li glassy phase enabled the intimate contact interface and uniform $\mathrm{Li}^{+}$flux. 


\section{Chapter 3: Research objectives and approach}

\subsection{Research objectives}

In this research work, the main objective is to comprehensively improve the electrochemical performance of Li-S battery by cathode materials design and Li metal anode modification, as schemed in Figure 16.

For the cathode side, sulfur electrodes will be mainly designed by integrating conductive matrix, porous host materials, chemical anchors, redox catalysts into the ensemble to enable the strong polysulfides adsorbability, fast redox kinetics, and structure stability, thus finally resulting in high reversible capacities, remarkable cycle stability, and excellent rate capability.

For the Li metal anode, two methods will be mainly conducted, i.e., (1) stabilizing the anode and-electrolyte interface by protective layer with high $\mathrm{Li}^{+}$conductivity to enable uniform $\mathrm{Li}$ deposition under the protective layer; (2) building three-dimensional conductive host materials for uniform Li deposition

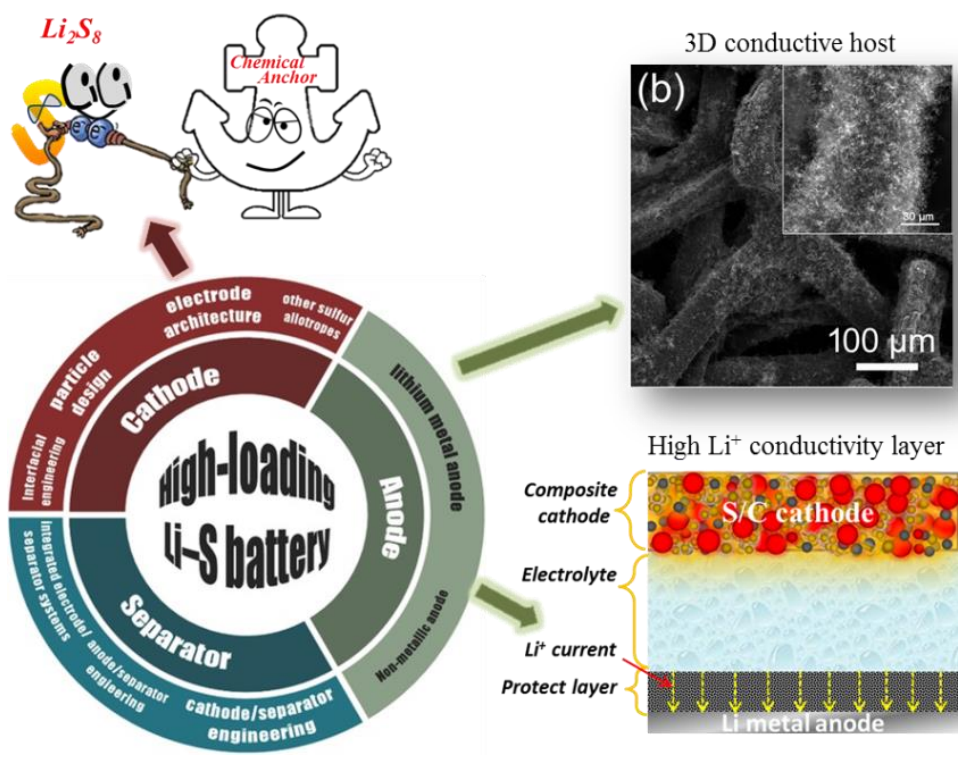

Figure 16 Research approaches based on the sulfur cathode and lithium metal anode to realize the objectives of high-performance Li-S batteries. 


\subsection{Characterization methods}

The phase structure characterization of the samples was obtained by PANalytical X'Pert Pro X-ray Diffractometer (XRD) using $\mathrm{Cu} \mathrm{K \alpha}$ radiation. The particle morphology and lattice structure of the samples were observed by a scanning electron microscope (SEM, Hitachi S-4700) and high resolution-transmission electron microscopy (HR-TEM, JEM-2100) equipped with Energy-dispersive X-ray spectroscopy (EDX). The surface chemical state was characterized by Digilab FTS 7000/UMA 600 Fourier transform infrared spectroscopy (FTIR), Raman spectroscopy (Renishaw InVia, excited by $532 \mathrm{~nm}$ He-Ne laser), and X-ray photoelectron spectroscopy (XPS, PHI VersaProbe 5000, Energy range: 0-1486.6 eV Binding Energy with Al $\mathrm{K} \alpha$ source). The surface area and pore size distribution were determined by the $\mathrm{N}_{2}$ adsorptiondesorption technique (Quantachrome Autosorb 2020). The mass ratio of sulfur in the composite can be analyzed by Thermogravimetric (TG, SDT Q600) analysis.

\subsection{Polysulfides trapping ability evaluation}

The polysulfide-trapping ability was performed by immersing the corresponding adsorbent in the $\mathrm{Li}_{2} \mathrm{~S}_{6}$ solution. $1 \mathrm{M} \mathrm{Li}_{2} \mathrm{~S}_{6}$ solution was prepared by adding $\mathrm{Li}_{2} \mathrm{~S}$ and sulfur at a molar ratio of 1:5 in DME stirring at $60{ }^{\circ} \mathrm{C}$ for $12 \mathrm{~h}$ in Ar. Samples with the same surface area were added to 5 $\mathrm{mL}$ of $\mathrm{Li}_{2} \mathrm{~S}_{6} / \mathrm{DME}$ solution separately. After vigorous magnetic stirring, the suspensions were centrifuged to obtain upper clear liquid before taking photographs. To demonstrate the chemical interaction between host material and polysulfides, XPS of the pristine and cycled (at discharge state, $2.2 \mathrm{~V}$ ) electrodes was conducted, respectively. The cells were disassembled in the glove box. The electrodes were washed carefully with DME to remove the electrolyte residues and dried for further characterization. 


\subsection{Electrochemical measurements}

The coin cells were assembled using the as-prepared designed electrode disks as the working electrode, lithium metal foil (MTI Corporation) as the counter electrode, and porous polypropylene (Celgard 2400) as a separator. The liquid electrolyte was $1 \mathrm{M}$ lithium bis(trfluoromethanesulfonyl) imide (LiTFSI) and $1 \% \mathrm{LiNO}_{3}$ dissolved in dioxolane (DOL) and dimethoxyethane (DME) (1:1 volume ratio). The cells (CR2032) were fabricated in an argonfilled glove box (moisture and oxygen levels less than 1ppm). The cycle performance and rate capability of the cells were tested by Arbin BT2143 32CH.

The cyclic voltammetry (CV) measurements were conducted with the Gamry Interface 5000E to identify the characteristic electrochemical reactions and to evaluate the $\mathrm{Li}^{+}$diffusion coefficient. Symmetric cells were also assembled to confirm the redox kinetics enhanced by metal sulfides through CV curves.

EIS measurements were performed to evaluate the interface behavior with the Gamry Interface $5000 \mathrm{E}$ at open-circuit potential with sinusoidal potential excitation of $5 \mathrm{mV}$ amplitude. The frequency range was from $1 \mathrm{MHz}$ to $0.1 \mathrm{~Hz}$. 


\section{Chapter 4: Nanosized $\mathrm{FeS}_{2}$ Particles Caged in the Hollow Carbon}

\section{Shell as a Robust Polysulfide Adsorbent and Redox Mediator}

\subsection{Introduction}

Pyrite $\left(\mathrm{FeS}_{2}\right)$ is one of the most promising candidates because of its high earth abundance and low-cost advantages. Zhang et al. demonstrated that $\mathrm{FeS}_{2}$ could chemically combine the LiPSs by forming $\mathrm{Li}_{2} \mathrm{FeS}_{2+n}$ complexes based on the redshift of Raman absorbance. ${ }^{[114]}$ To avoid compromising the sulfur loading, Yang et al. fabricated a FeS-stabilized porous carbon as sulfur host. ${ }^{[141]}$ The porous carbon as the major chamber for the sulfur host could physically confine LiPSs while FeS additive further chemically interacted with LiPSs through bonding with FeS. Very recently, Xi et al. synthesized a multifunctional $\mathrm{FeS}_{2} / \mathrm{FeS} / \mathrm{S}$ composite by ball-milling route for high volumetric capacity $\mathrm{Li}-\mathrm{S}$ batteries and illustrated that $\mathrm{FeS}_{2}$ showed a particularly stronger catalytic effect on the reduction reaction of the long-chain polysulfides than FeS based on the DFT calculation results. ${ }^{[142]}$ Although these methods provide new insights into the design of high-performance Li-S batteries, cycle stability, and rate capability are still unsatisfactory. Therefore, it is still a challenge to optimize the structure of composites with uniformly distributed active sulfur combined with sufficient polar chemical anchors and redox mediators to enable stable and longer cycle life, especially excellent rate capability of Li-S batteries.

To address the challenges in cathode development, here in this work, we facilely designed and prepared a cathode composite with nanosized $\mathrm{FeS}_{2}$ particles caged in hollow carbon shell as sulfur host, robust polysulfide adsorbent, and redox mediator for Li-S batteries application (FeS $\left.\mathrm{F}_{2} @ \mathrm{C}-\mathrm{S}\right)$. As shown in Figure 17, the hollow carbon cage can not only increase electronic conductivity but also facilitate Li-ion flux transportation. Therefore, excellent rate capability can 
be achieved due to this electrode kinetics advantage. In addition, the amorphous hollow carbon cage can act as a physical barrier or confinement to prevent the diffusion of polysulfides and to impede/accommodate the volume effects during the electrochemical process, which further helps to keep the cycle stability of the electrode. Moreover, the S-doped carbon formed during the sulfurization process can chemically adsorb the polysulfides through the C-S bonding. The sulfiphilic $\mathrm{FeS}_{2}$ nanoparticles, which intimately contact with active sulfur in the carbon cages, can provide static (polar-polar interaction) and dynamic (catalyzing the redox conversion) interaction between the host and polysulfides. Synergistically, this unique structure of composite integrating all functional units provides both effectively physical and chemical protection for the polysulfides. Therefore, excellent electrochemical performance can be achieved.

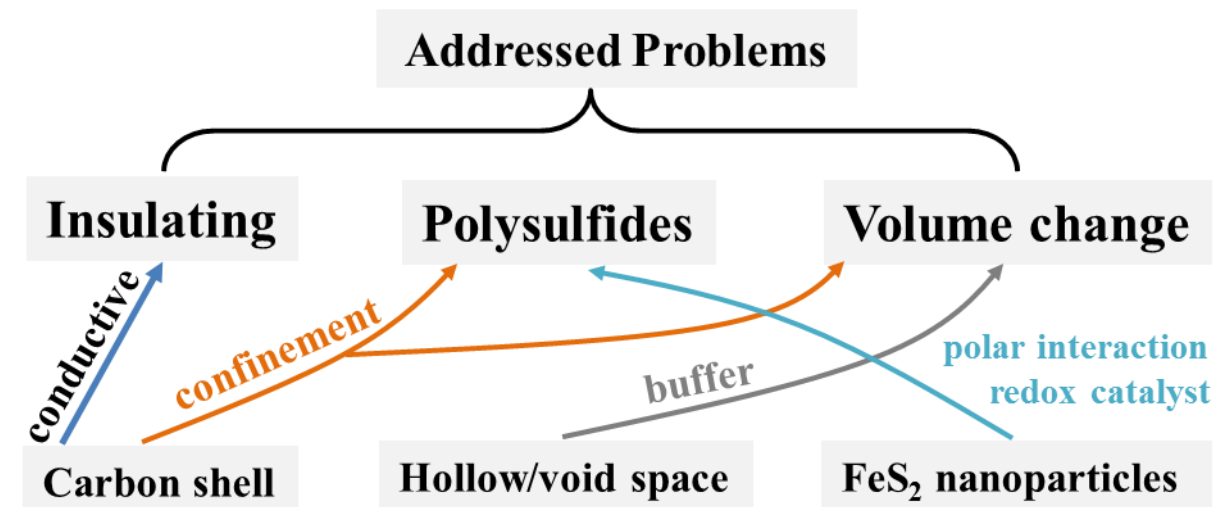

Figure 17 Design thought for sulfur electrode development.

\subsection{Synthesis of $\mathrm{FeS}_{2} @ \mathrm{C}-\mathrm{S}$ composite.}

$\mathrm{Fe}_{2} \mathrm{O}_{3}$ nanotubes were firstly prepared by a hydrothermal method based on a coordinationassisted dissolution mechanism reported by Jia et al. ${ }^{[143]}$ Simply, $\mathrm{FeCl}_{3}, \mathrm{NaH}_{2} \mathrm{PO}_{4}$, and $\mathrm{Na}_{2} \mathrm{SO}_{4}$ were dissolved into $80 \mathrm{~mL}$ of deionized (DI) water with concentrations of $0.02,3.26 \times 10^{-4}$, and $5.28 \times 10^{-4}\left(\mathrm{~mol} \mathrm{~L}^{-1}\right)$, respectively. After vigorous stirring, the yellow transparent solution was then transferred into a Teflon-lined stainless steel autoclave for hydrothermal treatment at $200{ }^{\circ} \mathrm{C}$ 
for $48 \mathrm{~h}$. The obtained precipitates were separated by centrifugation, washed with DI water and absolute ethanol, and then dried in air at $80{ }^{\circ} \mathrm{C}$ to get the final $\mathrm{Fe}_{2} \mathrm{O}_{3}$ nanotubes. The obtained $\mathrm{Fe}_{2} \mathrm{O}_{3}$ nanotubes were put into a quartz tube $(50 \mathrm{~mm}$ diameter $)$ for the chemical vapor deposition (CVD) process. The temperature was heating at $5{ }^{\circ} \mathrm{C} \min ^{-1}$ up to $800{ }^{\circ} \mathrm{C}$ and maintained for $1 \mathrm{~h}$. The toluene as the carbon source was carried by $5 \% \mathrm{H}_{2} / \mathrm{Ar}$ at a flow rate of $0.1 \mathrm{~L} \mathrm{~min}^{-1}$ (Figure 18a). Figure $18 b$ illustrates the subsequently synthetic route of $\mathrm{FeS}_{2} @ \mathrm{C}-\mathrm{S}$ composite. After the CVD process, $0.1 \mathrm{~g}$ of the obtained black powder $\left(\mathrm{Fe}_{3} \mathrm{O}_{4} @ \mathrm{C}\right)$ mixed with $0.5 \mathrm{~g}$ sulfur was sealed in a glass tube under the protection of $\mathrm{Ar}$ gas and then heated at $500{ }^{\circ} \mathrm{C}$ for $1 \mathrm{~h}$ to enable the sulfurization process $\left(\mathrm{FeS}_{2} @ \mathrm{C}\right)$. Sulfur impregnation was conducted at $155{ }^{\circ} \mathrm{C}$ for $24 \mathrm{~h}$ by mixing $\mathrm{FeS}_{2} @ \mathrm{C}$ and sulfur with a mass ratio of 1:3. As a comparison, commercial mesoporous carbon (MC) was also employed as a sulfur host. MC was sufficiently mixed with sulfur at a mass ratio of $1: 3$ by hand-milling. The mixture was then maintained in the quartz tube at $155^{\circ} \mathrm{C}$ for $24 \mathrm{~h}$ under the Ar to form MC/S composite.
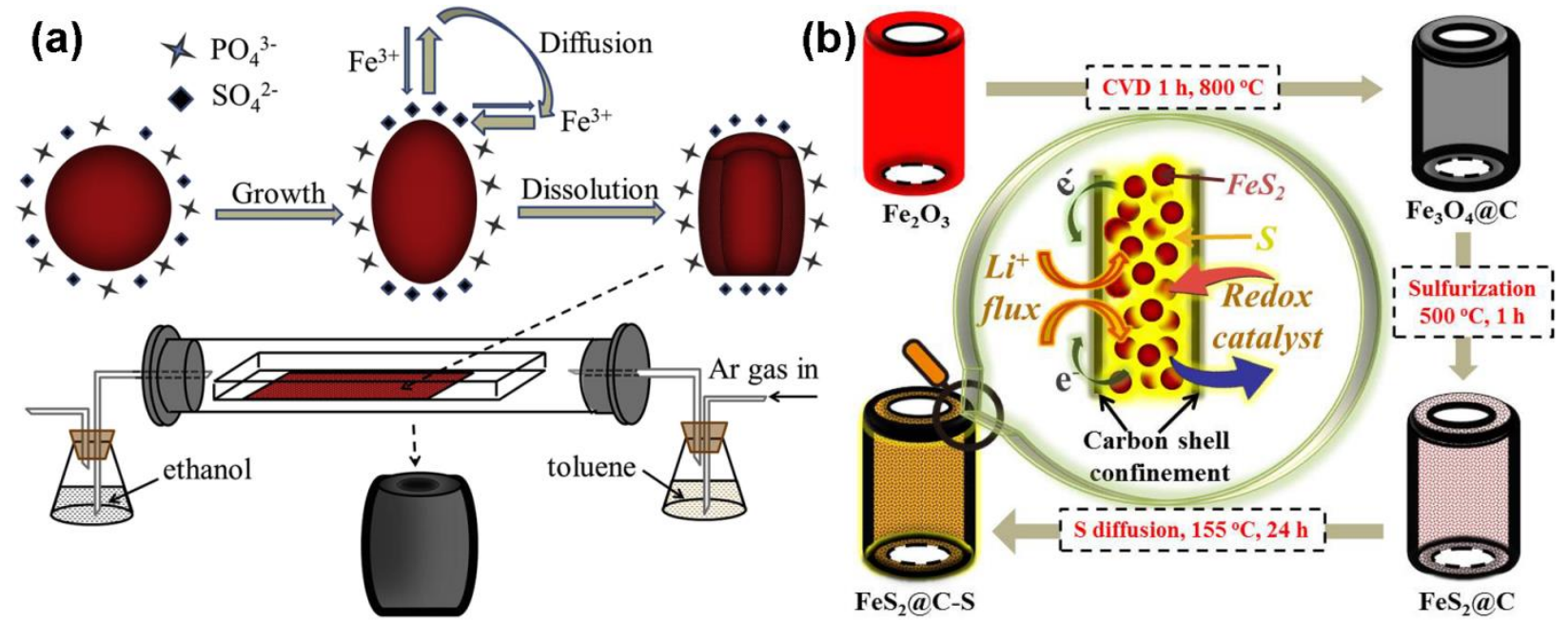

Figure 18 (a) formation mechanism of $\mathrm{Fe}_{2} \mathrm{O}_{3}$ nanotube and CVD apparatus. ${ }^{[144]}$. (b) The schemed synthesis process of $\mathrm{FeS}_{2} @ \mathrm{C}-\mathrm{S}$ composite with the mechanism of high $\mathrm{Li}^{+} / \mathrm{e}^{-}$flux and redox conversion kinetics. 


\subsection{Phase and morphology characterization}

Figure 19a shows the XRD pattern of $\mathrm{FeS}_{2} @ \mathrm{C}-\mathrm{S}$ composite. The reflection peaks coincide well with the standard PDF cards of $\mathrm{FeS}_{2}$ (JCPDS No. 42-1340) and S (JCPDS No. 08-0247), respectively, indicating that the incorporated sulfur is orthorhombic and presents the primary crystalline phase. The corresponding XRD patterns for samples at different synthesis steps were illustrated in Figure 19b. The carbon content in the $\mathrm{FeS}_{2} @ \mathrm{C}$ host was determined by the TGA method under an air atmosphere. As shown in Figure 19c, the significant weight loss occurs between $300{ }^{\circ} \mathrm{C}$ to $700{ }^{\circ} \mathrm{C}$, which should be due to the oxidation of carbon to $\mathrm{CO}_{2} / \mathrm{CO}$ and the oxidation of $\mathrm{FeS}_{2}$ to $\mathrm{Fe}_{2} \mathrm{O}_{3}$. From the total weight loss of 38.4 wt. \% and by simple calculation, it can be inferred that the percentage of carbon content in the $\mathrm{FeS}_{2} @ \mathrm{C}$ host is about 7.6 wt. \%. The sulfur ratio in the composite can be analyzed by the TGA curve in Figure 19d. The weight loss due to the evaporation of sulfur from the $\mathrm{FeS}_{2} @ \mathrm{C}$ host can be observed when the temperature is elevated to about $150{ }^{\circ} \mathrm{C}$ and becomes stable when the temperature increases to over $300{ }^{\circ} \mathrm{C}$. The approximate mass ratio of sulfur in the composite is estimated to $70 \mathrm{wt} . \%$. 

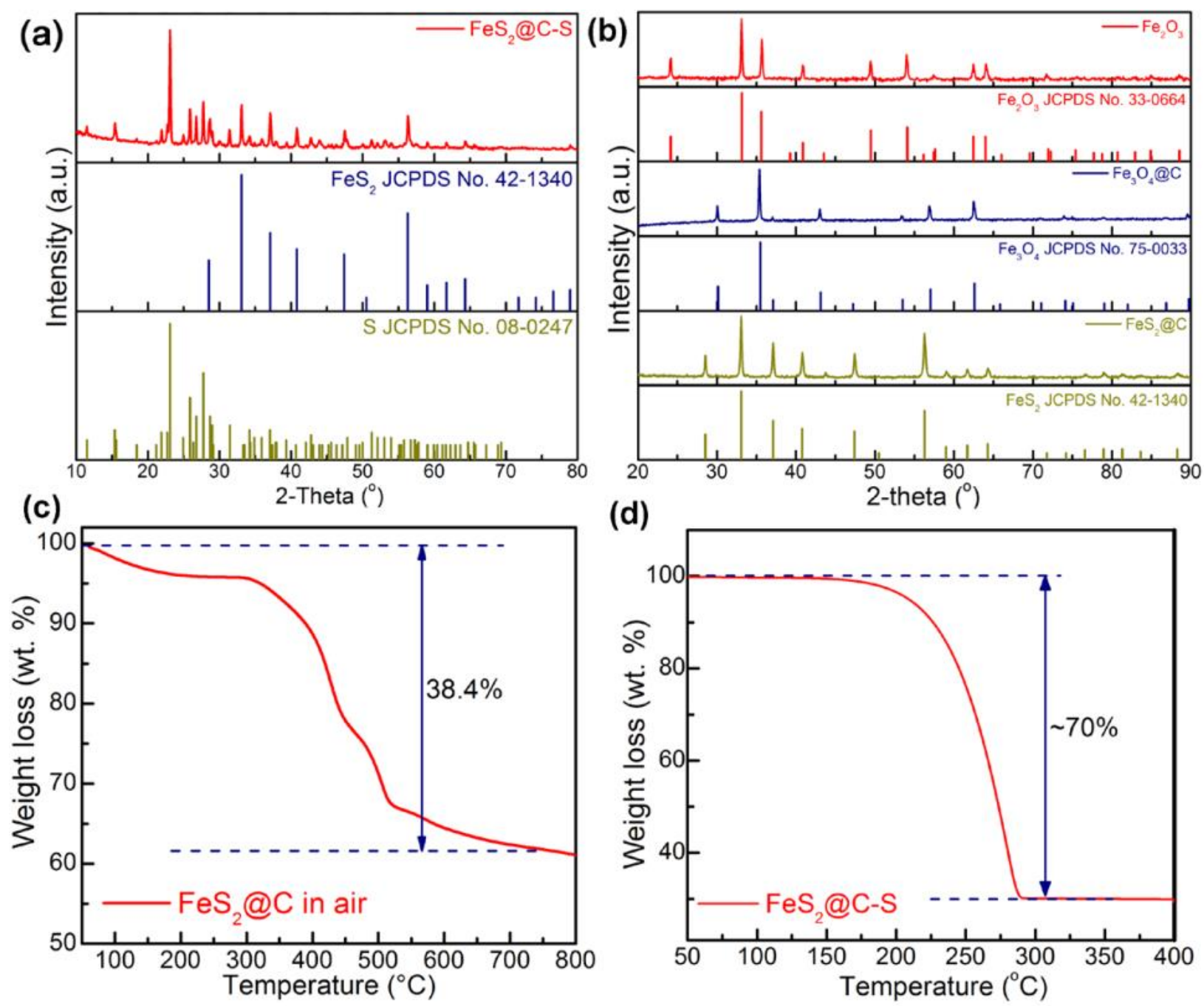

Figure 19 (a) XRD pattern of $\mathrm{FeS}_{2} @ \mathrm{C}-\mathrm{S}$ composite and standard XRD card of $\mathrm{FeS}_{2}$ and $\mathrm{S}$. (b) XRD patterns of the pristine $\mathrm{Fe}_{2} \mathrm{O}_{3}$ nanotubes obtained from the hydrothermal process, $\mathrm{Fe}_{3} \mathrm{O}_{4} @ \mathrm{C}$, and $\mathrm{FeS}_{2} @ \mathrm{C}$ with corresponding standard PDF cards. TG curve of $\mathrm{FeS}_{2} @ \mathrm{C}$ (c) and $\mathrm{FeS}_{2} @ \mathrm{C}-\mathrm{S}$ (d) composite recorded under air, $\mathrm{N}_{2}$ atmosphere, respectively.

Figure 20a and $\mathrm{b}$ show the SEM images of $\mathrm{FeS}_{2} @ \mathrm{C}-\mathrm{S}$ composite. Compare with the pristine $\mathrm{Fe}_{2} \mathrm{O}_{3}$ nanotubes (Figure 21a and b), the hollow structure of $\mathrm{FeS}_{2} @ \mathrm{C}-\mathrm{S}$ can be well maintained after CVD, sulfurization, and sulfur diffusion process without any bulk sulfur aggregated on the surface. CVD treatment enabled both the carbon deposition and reduction of 
$\mathrm{Fe}_{2} \mathrm{O}_{3}$ to form carbon-coated $\mathrm{Fe}_{3} \mathrm{O}_{4}\left(\mathrm{Fe}_{3} \mathrm{O}_{4} @ \mathrm{C}\right)$. As shown in Figure 21c, the carbon-veil overlayer can be clearly observed. The subsequent sulfurization at $500{ }^{\circ} \mathrm{C}$ for $1 \mathrm{~h}\left(\mathrm{FeS}_{2} @ \mathrm{C}\right)$ and sulfur incorporation at $155{ }^{\circ} \mathrm{C}$ for $24 \mathrm{~h}\left(\mathrm{FeS}_{2} @ \mathrm{C}-\mathrm{S}\right)$ would not destroy the hollow structure although some carbon fragment can be formed (Figure 21d). The high magnification SEM image of $\mathrm{FeS}_{2} @ \mathrm{C}-\mathrm{S}$ in Figure 20b obviously depicts the nanotube morphology with the outer diameter at $150 \sim 180 \mathrm{~nm}$ and the inner diameter at $60 \sim 80 \mathrm{~nm}$. Sulfur mainly incorporated into the void space between the $\mathrm{FeS}_{2}$ nanoparticles. This conclusion can be further confirmed by TEM images. The $\mathrm{FeS}_{2}$ nanoparticles with size ranging from 50 100 nm are well caged in the hollow carbon shell (Figure 20c). In addition, the void space between the refined $\mathrm{FeS}_{2}$ nanoparticles can be clearly identified, which provides many effective sites for sulfur loading and intimate contact with active sulfur. After the diffusion process at $155^{\circ} \mathrm{C}$ for 24 hours, the void space can be filled with the sulfur, resulting in the relatively blurred particle surface due to the insulating nature of sulfur (Figure 20e). The corresponding EDX results reveal that the atomic ratio of $\mathrm{S}$ to $\mathrm{Fe}$ in $\mathrm{FeS}_{2} @ \mathrm{C}-\mathrm{S}$ (Figure 20f) is much higher than that in $\mathrm{FeS}_{2} @ \mathrm{C}$ (close to 2:1, Figure 20d), demonstrating that sulfur has been successfully embedded into the $\mathrm{FeS}_{2} @ \mathrm{C}$ host. Figure 20g shows the HR-TEM image of $\mathrm{FeS}_{2} @ \mathrm{C}-\mathrm{S}$ composite. Besides the amorphous carbon layer with the thickness of $\sim 5 \mathrm{~nm}$, the well-resolved crystalline structure of $\mathrm{FeS}_{2}$ and sulfur can also be detected, from which the lattice fringes of 0.268 and $0.392 \mathrm{~nm}$ can be assigned to the (200) plane of $\mathrm{FeS}_{2}$ and the (222) plane of sulfur, respectively. It can be demonstrated from Figure 20g that the carbon shell has been successfully coated onto the $\mathrm{FeS}_{2}$ nanoparticles by using $\mathrm{Fe}_{2} \mathrm{O}_{3}$ as a template with subsequently CVD treatment. In addition, the active sulfur can be mainly impregnated into the void space between $\mathrm{FeS}_{2}$ nanoparticles and further confined in the hollow carbon cages. The Brunauer-Emmett-Teller (BET) surface area was calculated by applying the 
linear part of the BET plot. The corresponding BET surface area of $\mathrm{FeS}_{2} @ \mathrm{C}$ composite significantly decreases from 20.8 to $7.4 \mathrm{~m}^{2} \mathrm{~g}^{-1}$ after sulfur impregnation (Figure 20h). The distribution of pores size was analyzed by the Barrett-Joyner-Halenda (BJH) model. As shown in Figure 20i, an obviously decreased adsorption volume in the mesoporous scale range between 2 to $65 \mathrm{~nm}$ can be observed. Significant adsorption volume change occurs between 2 to $15 \mathrm{~nm}$, as illustrated in the inset of Figure 20i. Therefore, it can be concluded that the active sulfur is mainly filled in the mesopores resulting from the void space between $\mathrm{FeS}_{2}$ nanoparticles, as also confirmed by the TEM results in Figure 20c and e. The uniformly distributed sulfur in the $\mathrm{FeS}_{2} @ \mathrm{C}$ host can be confirmed by the corresponding EDX elemental mapping. As displayed in Figure 22, the $\mathrm{FeS}_{2} @ \mathrm{C}-\mathrm{S}$ particles with a refined hollow structure can be clearly distinguished. The Fe (Figure 22c) and S (Figure 22d) are homogeneously dispersed in the carbon matrix (Figure 22b) without any notable segregation, indicating the sulfur can be effectively encapsulated in the hollow carbon shell in which $\mathrm{FeS}_{2}$ nanoparticles are caged. 

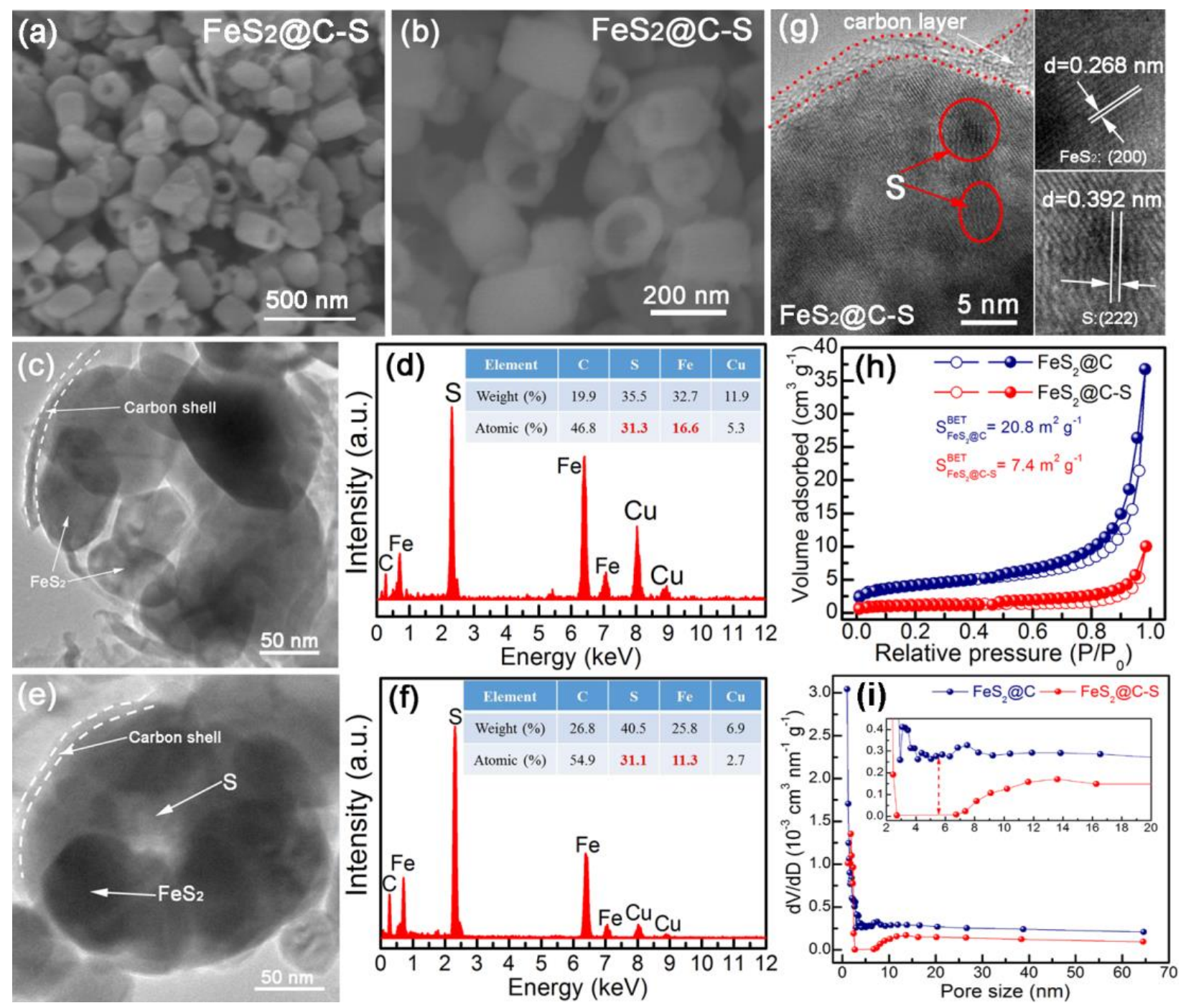

Figure 20 SEM images of low magnification (a) and high magnification (b) of prepared $\mathrm{FeS}_{2} @ \mathrm{C}-\mathrm{S}$ composite. TEM images of $\mathrm{FeS}_{2} @ \mathrm{C}(\mathrm{c})$ and $\mathrm{FeS}_{2} @ \mathrm{C}-\mathrm{S}$ (e) with corresponding EDX results (d, f), respectively. (g) HR-TEM micrographs of the $\mathrm{FeS}_{2} @ \mathrm{C}-\mathrm{S}$ composite. (h) $\mathrm{N}_{2}$ adsorption/desorption isotherms and (i) pore size distribution of the corresponding samples. 


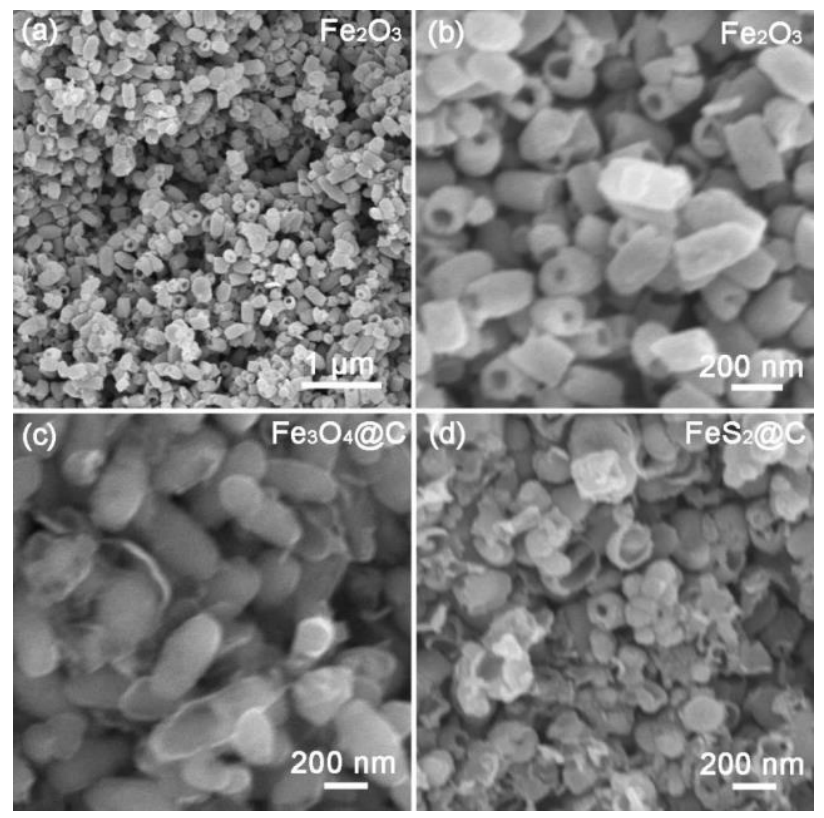

Figure 21 SEM images of pristine $\mathrm{Fe}_{2} \mathrm{O}_{3}$ nanotubes at low (a) and high magnification (b), $\mathrm{Fe}_{3} \mathrm{O}_{4} @ \mathrm{C}(\mathrm{c})$, and $\mathrm{FeS}_{2} @ \mathrm{C}(\mathrm{d})$.

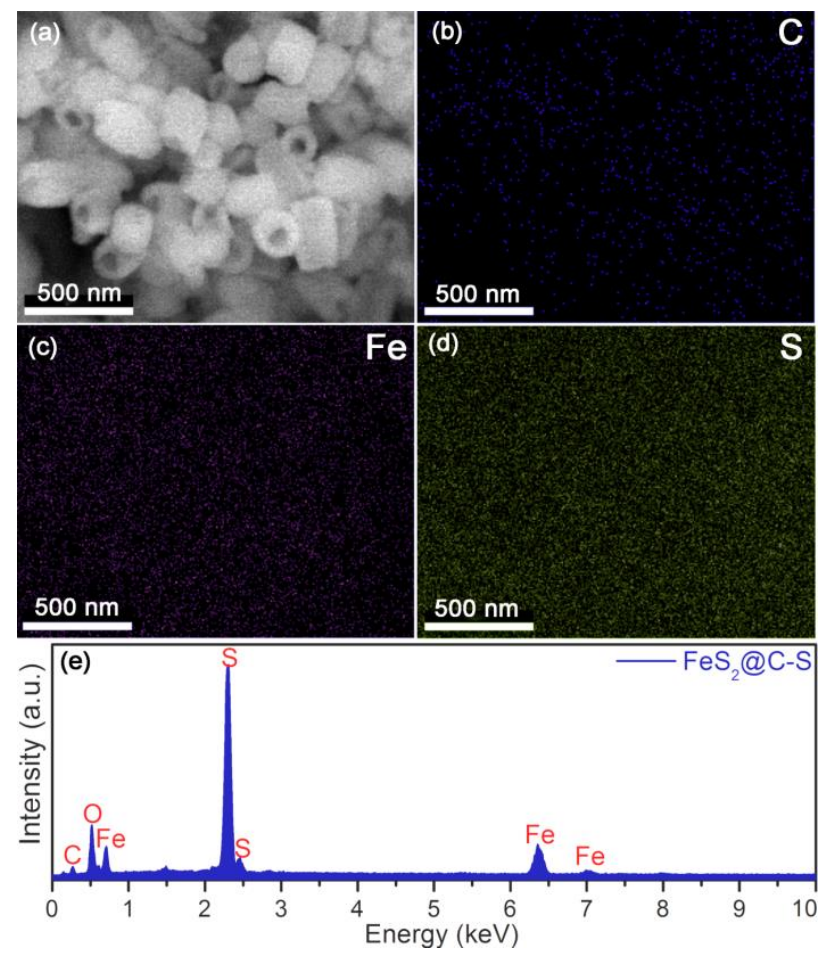

Figure 22 SEM images of $\mathrm{FeS}_{2} @ \mathrm{C}-\mathrm{S}$ and corresponding elemental maps of $\mathrm{C}$ (b), $\mathrm{Fe}$ (c), and $\mathrm{S}$ (d) and its EDX spectrum. 


\subsection{Polysulfides trapping ability}

To demonstrate the polysulfide trapping capability, a visualized adsorption experiment was conducted to show the advantage of $\mathrm{FeS}_{2} @ \mathrm{C}$ host. After one week stirring to ensure the sufficient adsorption, the solution involved $\mathrm{FeS}_{2} @ \mathrm{C}$ becomes colorless, while it still keeps brown for MC adsorbent (Figure 23a), indicative of the excellent capability of $\mathrm{FeS}_{2} @ \mathrm{C}$ in polysulfides immobilization. The "chemical anchors" within $\mathrm{FeS}_{2} @ \mathrm{C}-\mathrm{S}$ composite especially associated with the chemical adsorption towards LiPSs were further investigated by FTIR, Raman, and XPS. Due to the weak reaction between $\mathrm{Fe}_{2} \mathrm{O}_{3}$ and FTIR, only a small peak at $\sim 570$ $\mathrm{cm}^{-1}$ can be observed due to the Fe-O stretching vibration. As to the $\mathrm{FeS}_{2} @ \mathrm{C}-\mathrm{S}$ composite (Figure 23b), the typical peaks at 1575 can be ascribed to the $\mathrm{C}=\mathrm{C}$ stretching vibrations originating from the graphite planar. ${ }^{[145]}$ The peak at $\sim 1150 \mathrm{~cm}^{-1}$ is assigned to symmetric stretching vibrations of $-\mathrm{COOH}$ groups on the pyrolysis carbon. ${ }^{[145 b, 146]}$ The characteristic peaks at $\sim 1070$ and $\sim 820 \mathrm{~cm}^{-1}$ can be due to the O-S stretching modes, resulting from the active sites bonding with the short sulfur chains. In the Raman spectrum (Figure 23c), two prominent peaks at 1376 and $1585 \mathrm{~cm}^{-1}$ corresponding to the $\mathrm{D}$ and $\mathrm{G}$ bands are well-documented in the previous literature. ${ }^{[147]}$ Meanwhile, another three peaks (inset of Figure 23c) located at 336, 372, and 428 $\mathrm{cm}^{-1}$ correspond to the typical $\mathrm{E}_{\mathrm{g}}\left(\mathrm{S}_{2}\right.$ vibration), $\mathrm{A}_{\mathrm{g}}\left(\mathrm{S}-\mathrm{S}\right.$ in phase stretch), and $\mathrm{T}_{\mathrm{g}}$ (coupled vibration and stretch) modes of $\mathrm{FeS}_{2}$ crystal, respectively, ${ }^{[148]}$ indicating the successful introduction of carbon coating and sulfurization process to form $\mathrm{FeS}_{2} @ \mathrm{C}$ host. XPS was further conducted to illustrate the active adsorption sites and the possible interactions between polysulfides and $\mathrm{FeS}_{2} @ \mathrm{C}$ host. For pristine $\mathrm{FeS}_{2} @ \mathrm{C}-\mathrm{S}$, the survey spectrum displays typical peaks for $\mathrm{C}, \mathrm{S}, \mathrm{Fe}$, and $\mathrm{O}$ elements. The $\mathrm{C} 1 s \mathrm{XPS}$ spectrum can be deconvoluted into four peaks (Figure 23d). The typical peak at $284.8 \mathrm{eV}$ and $287.5 \mathrm{eV}$ can be ascribed to $s p^{2}$ hybridized 
carbon and $\mathrm{C}-\mathrm{O}$ species, respectively. The peak at $286.3 \mathrm{eV}$ corresponding to $\mathrm{C}-\mathrm{S}$ bonds proves the covalent bonding between sulfur and carbon shell in the composite. ${ }^{[94,149]}$ Another small shoulder peak at $283.2 \mathrm{eV}$ corresponds to the $\mathrm{Fe}-\mathrm{C}$ bond resulting from the $\mathrm{Fe}_{3} \mathrm{C}$ formed during the CVD process. ${ }^{[150]}$ The Fe $2 p_{3 / 2}$ and $2 p_{1 / 2}$ peaks at 708.1 and $721.7 \mathrm{eV}$ are coupled with their satellite peaks at 713.2 and $727.5 \mathrm{eV}$ (Figure 23e), respectively, consistent with those of reported $\mathrm{FeS}_{2} \cdot{ }^{[142,148 \mathrm{a}, 148 \mathrm{c}]}$ This also confirms the successful sulfurization process from $\mathrm{Fe}_{3} \mathrm{O}_{4} @ \mathrm{C}$ to form $\mathrm{FeS}_{2} @ \mathrm{C}$ at $500{ }^{\circ} \mathrm{C}$. The $\mathrm{S} 2 p$ spectrum (Figure 23f) demonstrates two peaks centered at 168.8 and $167.4 \mathrm{eV}$, which can be assigned to the sulfates and sulfites species resulting from the adventitious oxidation of the surface during the sulfurization process. ${ }^{[102,147 e, 151]}$ As sulfur and $\mathrm{FeS}_{2}$ are both included in the electrode, there should be two $2 p_{3 / 2} / 2 p_{1 / 2}$ doublets in the $\mathrm{S} 2 p$ spectrum. The peaks at $163.2 \mathrm{eV}$ and $162.5 \mathrm{eV}$ can be attributed to the spin-orbit coupling, but the binding energy of the $\mathrm{S} 2 p_{3 / 2}$ peak $(162.5 \mathrm{eV})$ is much lower than that of elemental sulfur $(164.0 \mathrm{eV})$ due to the covalently bonded carbon and sulfur species. The other two peaks at 161.7 $\mathrm{eV}$ and $160.1 \mathrm{eV}$ should be reasonably attributed to $\mathrm{FeS}_{2}$, which also coincides with the reported literature. ${ }^{[152]}$ Figure $23 \mathrm{~g}$ shows the XPS peak of the O $1 s$ core level of fresh $\mathrm{FeS}_{2} @ \mathrm{C}-\mathrm{S}$ electrode film, which is deconvoluted into three peaks. The peak at $533.6 \mathrm{eV}$ can be due to the trace $\mathrm{H}_{2} \mathrm{O}$ chemically adsorbed on the surface; while the other peaks at $531.3 \mathrm{eV}$ and $530.2 \mathrm{eV}$ can be due to the $\mathrm{C}=\mathrm{O}$ groups in the aromatic ring and $\mathrm{C}-\mathrm{O}$ bonds, respectively. This $\mathrm{C}-\mathrm{O}$ binding energy is slightly lower than the reported values, indicating that the $\mathrm{O}$ atoms are possibly sulfurized to form the C-O-S bonds as functional groups. ${ }^{[95,153]}$ Comprehensively, the hollow structured composite with sufficiently built "chemical anchors" has been elaborately designed to enable the strong chemical adsorption towards polysulfides and fast kinetics of redox reactions between the sulfur species. 

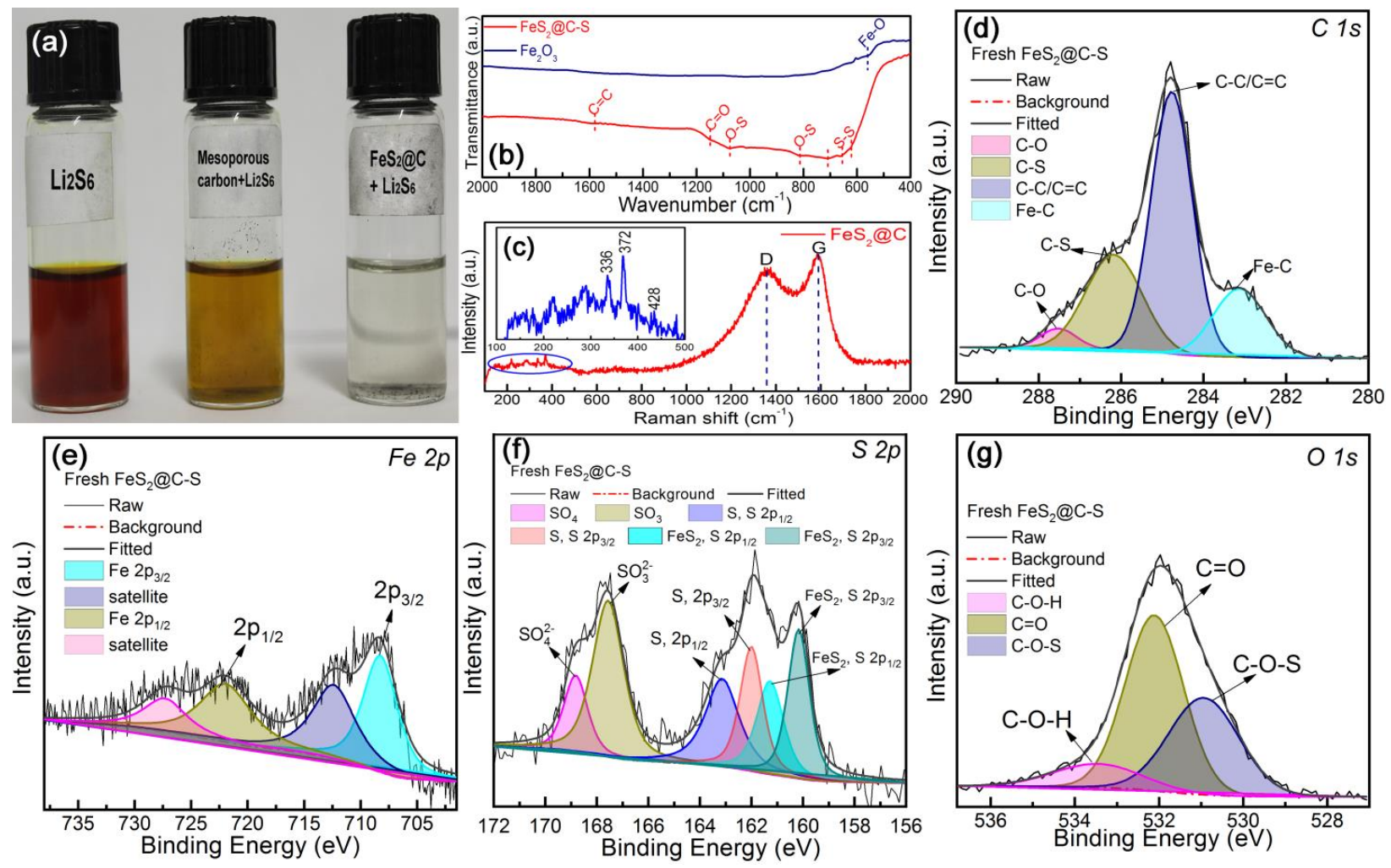

Figure 23 (a) Adsorption ability test of $\mathrm{MC}$ and $\mathrm{FeS}_{2} @ \mathrm{C}-\mathrm{S}$ electrodes with the representative lithium polysulfides (1 $\mathrm{M} \mathrm{Li}_{2} \mathrm{~S}_{6}$ ). (b) FTIR and (c) Raman curves for various samples, and highresolution XPS spectra of (d) C $1 s$, (e) Fe $2 p$, (f) S $2 p$, and (g) O $1 s$ peaks of fresh $\mathrm{FeS}_{2} @ \mathrm{C}-\mathrm{S}$ electrode film.

\subsection{Redox kinetics of polysulfides}

In addition to the potentially strong adsorption ability towards polysulfides due to the active chemical anchors, moreover, the electrocatalytic role of $\mathrm{FeS}_{2}$ in facilitating the redox reaction of polysulfides has also been demonstrated and detailedly analyzed. Figure 24a and b show the typical CV curves of the $\mathrm{FeS}_{2} @ \mathrm{C}-\mathrm{S}$ and $\mathrm{MC} / \mathrm{S}$ electrodes at a scan rate of $0.1 \mathrm{mV} \mathrm{s}^{-1}$ between 1.7 and 3.0 V. Both of them show two cathodic peaks and two anodic peaks (overlapped for $\mathrm{MC} / \mathrm{S}$ electrode). Obviously, the cathodic and anodic peaks of the $\mathrm{FeS}_{2} @ \mathrm{C}-\mathrm{S}$ electrode are much sharper and narrower with higher current, indicative of fast kinetics and stable redox reactions. 
To further reveal the electrocatalytic effects, the peak voltages of $\mathrm{FeS}_{2} @ \mathrm{C}-\mathrm{S}$ and MC/S electrodes for two cathodic peaks (I and II) and one anodic peak (III) derived from their CV curves are compared in Figure 24c. The presence of $\mathrm{FeS}_{2}$ can raise the discharge voltages of cathodic peak I and II by $40 \mathrm{mV}$ and $120 \mathrm{mV}$, respectively, and reduce the charge voltage of anodic peak by $230 \mathrm{mV}$. The onset potentials were also compared in Figure 24d, which was taken at a current density of $10 \mu \mathrm{A} \mathrm{cm}{ }^{-2}$ beyond the baseline current. ${ }^{[112]}$ Similarly, $\mathrm{FeS}_{2}$ contributes to the increased onset potentials of cathodic peaks (I and II) and decreased onset potential of anodic peak (III), indicating the earlier start point of redox reactions due to the electrocatalytic role of $\mathrm{FeS}_{2}$. Such an electrocatalytic effect of $\mathrm{FeS}_{2}$ on promoting kinetics of redox reactions can be illustrated more evidently by comparing their polarization curves (Figure $24 \mathrm{e}$ and f) with the corresponding Tafel plots (Figure $24 \mathrm{~g}$ ). $\mathrm{FeS}_{2}$ shows much smaller Tafel slopes of 104 and $80 \mathrm{mV} \mathrm{dec}^{-1}$ in the reduction (I) and oxidation (III) processes than those of MC/S counterpart. In addition, the $\mathrm{FeS}_{2} @ \mathrm{C}$ host enabled fast redox kinetics and lower charge transfer can be further confirmed by CV (Figure 24h, scan rate: $10 \mathrm{mV} \mathrm{s}^{-1}$ ) and EIS (Figure 24i) results in symmetric cells. Obviously, the current density of $\mathrm{FeS}_{2} @ \mathrm{C}$ symmetric cells is much higher than that of the MC control sample. The significantly lowered charge transfer resistance $\left(\mathrm{R}_{\mathrm{ct}}\right)$ of $119.7 \Omega \mathrm{cm}^{2}$ for $\mathrm{FeS}_{2} @ \mathrm{C}$ symmetric cell compared to that of MC $\left(665.7 \Omega \mathrm{cm}^{2}\right)$ in the Nyquist plots further confirm the boosted charge transfer process at $\mathrm{Li}_{2} \mathrm{~S}_{6} / \mathrm{FeS}_{2} @ \mathrm{C}$ interface. Detailed equivalent circuit and fitting results are shown in Figure 25 and Table 4. The electrochemical behavior analysis significantly verifies the notable enhancement of the redox reactions by $\mathrm{FeS}_{2} @ \mathrm{C}$ host, including accelerating kinetics, promoting redox reversibility and stability, and mitigating polarization. Thus, it can be concluded that the sulfiphilic $\mathrm{FeS}_{2}$ nanoparticles caged in the electrically conductive hollow carbon shell as a highly efficient 
electrocatalyst can trigger the LiPSs redox reactions with fast kinetics by promoting the diffusion of polysulfide ions and $\mathrm{Li}^{+} / \mathrm{e}^{-}$transfer to $\mathrm{LiPSs} / \mathrm{FeS}_{2}$ interface, reducing the energy barriers. ${ }^{[12]}$ Collectively, all these electrochemical results evidence that $\mathrm{FeS}_{2}$ plays pivotal roles in electrocatalytically decreasing the energy barriers and facilitates the LiPSs-involved redox reactions.
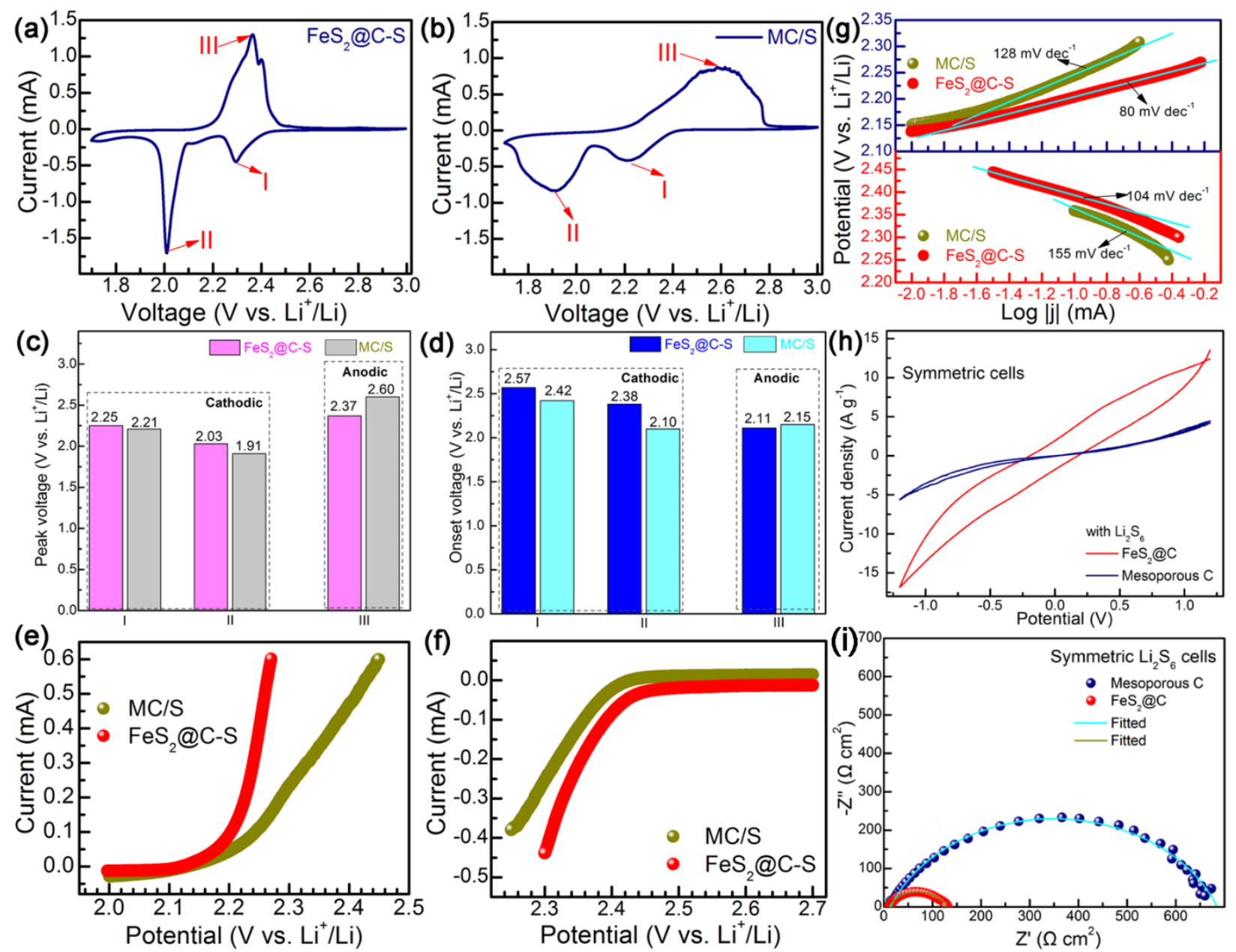

Figure 24 Typical CV curves of $\mathrm{FeS}_{2} @ \mathrm{C}-\mathrm{S}$ (a) and MC/S (b) cathodes. Comparison of CV peak voltages (c), onset potentials (d), cathodic (e) and anodic (f) polarization curves and Tafel plots (g) of asymmetrical Li-S cells consisting of both $\mathrm{FeS}_{2} @ \mathrm{C}-\mathrm{S}$ or MC/S cathode and Li anode. (h) $\mathrm{CV}$ and (g) EIS Nyquist curves of symmetric cells of $\mathrm{FeS}_{2} @ \mathrm{C}$ or MC host electrodes. 


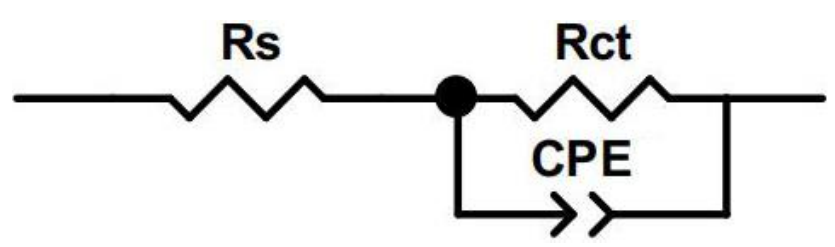

Figure 25 Equivalent circuit used for fitting the EIS spectra of symmetrical cells in Figure 5i and the corresponding fitting results.

Table 4 Fitting results of the equivalent circuit.

\begin{tabular}{ccccc}
\hline Cathode matrix & $\operatorname{Rs}\left(\boldsymbol{\Omega} \mathbf{c m}^{2}\right)$ & $\operatorname{Rct}\left(\boldsymbol{\Omega} \mathbf{c m}^{2}\right)$ & $\mathbf{C P E}-\mathbf{T}\left(\mathbf{\Omega}^{-\mathbf{1}} \mathbf{S}^{\mathbf{n}}\right)$ & CPE-P \\
\hline MC & 13.92 & 665.7 & $1.04 \times 10^{-5}$ & 0.78 \\
\hline FeS $_{2} @ \mathbf{C}-\mathbf{S}$ & 10.18 & 119.7 & $3.38 \times 10^{-5}$ & 0.74 \\
\hline
\end{tabular}

\subsection{Electrochemical performance}

With the strong polysulfides trapping capability by the polar-polar interaction resulting from the "chemical anchors" and the fast kinetics of redox reactions enabled by $\mathrm{FeS}_{2}$ electrocatalyst, the excellent electrochemical performance of $\mathrm{FeS}_{2} @ \mathrm{C}-\mathrm{S}$ electrode can be achieved. The $\mathrm{FeS}_{2} @ \mathrm{C}-\mathrm{S}$ electrode cycled at a current density of $0.2 \mathrm{C}$ can maintain a high specific capacity (ca. $800 \mathrm{mAh} \mathrm{g}^{-1}$ ) with the Coulombic efficiency close to $100 \%$ after 200 cycles (Figure 26a). The relatively low Columbic efficiency during the first 10 cycles should be ascribed to the shuttle effect caused by the residual sulfur on the surface. ${ }^{[154]}$ It should be noted that the $\mathrm{FeS}_{2} @ \mathrm{C}$ host contributes limited reversible capacity within the potential window of 1.7-2.6 V. By contrast, the capacity of MC/S electrode in Figure 27a shows a seriously fading to $\sim 200 \mathrm{mAh} \mathrm{g}^{-1}$ only after 50 cycles since the non-polar mesoporous carbon host cannot effectively suppress the polysulfide shuttle effect during cycling. The corresponding charge/discharge curves of MC/S and $\mathrm{FeS}_{2} @ \mathrm{C}-\mathrm{S}$ electrodes are shown in Figure 27b and c, respectively. Both electrodes show two discharge plateaus and charge plateaus, which coincides with the CV results in Figure 24. The 
discharge plateaus at $\sim 2.3$ and $\sim 2.0 \mathrm{~V}$ can be ascribed to the formation of long-chain polysulfides $\left(\mathrm{Li}_{2} \mathrm{~S}_{\mathrm{x}}, 4 \leq \mathrm{x} \leq 8\right)$ and then to insoluble short-chain discharged products $\mathrm{Li}_{2} \mathrm{~S}_{2} / \mathrm{Li}_{2} \mathrm{~S}$, respectively. Two charge plateaus appear at $\sim 2.3$ and $\sim 2.4 \mathrm{~V}$, accounting for the oxidation of lithium sulfides to LiPSs and sulfur. Remarkably, the charge/discharge curves of the FeS ${ }_{2} @ \mathrm{C}-\mathrm{S}$ electrode (Figure $27 \mathrm{c})$ are much better overlapped with relatively stable electrochemical polarization $(\Delta \mathrm{E})$, indicating the highly reversible ability of redox reactions. However, the polarization of the MC/S electrode (Figure 27b) significantly increases with cycling, showing sluggish redox conversion kinetics.

The superior rate performance of $\mathrm{FeS}_{2} @ \mathrm{C}-\mathrm{S}$ cathodes was also evaluated at different current densities, as displayed in Figure 26b. The specific capacities of the $\mathrm{FeS}_{2} @ \mathrm{C}-\mathrm{S}$ electrode decrease with increasing the charge/discharge rates. The reversible capacities of the battery using the $\mathrm{FeS}_{2} @ \mathrm{C}-\mathrm{S}$ as cathode electrodes are 872.6, 718.3, and $688.3 \mathrm{mAh} \mathrm{g}^{-1}$ at $0.5,1$, and $2 \mathrm{C}$, respectively. Even at a much higher current density of $5 \mathrm{C}$, the $\mathrm{FeS}_{2} @ \mathrm{C}-\mathrm{S}$ electrode can still retain a capacity of $500.7 \mathrm{mAh} \mathrm{g}^{-1}$. When the current density was abruptly switched back to 0.5 $\mathrm{C}$, the specific capacity can be able to return to $\sim 960 \mathrm{mAh} \mathrm{g}^{-1}$. The corresponding charge/discharge curves at various current densities are presented in Figure 26c. It should be noticed that the polarization $(\Delta \mathrm{E})$ significantly increases with the current densities changing from 0.5 to 5 C. Remarkably, the characteristic stable and phased plateaus can still be observed even at $5 \mathrm{C}$, reflecting the outstanding redox kinetics of $\mathrm{FeS}_{2} @ \mathrm{C}-\mathrm{S}$ electrode. On the contrary, the MC/S electrode shows fast capacity decay when increasing the current densities, only $~ 120 \mathrm{mAh}$ $\mathrm{g}^{-1}$ can be maintained at $5 \mathrm{C}$ (Figure 27d). The specific capacity of the MC/S electrode cannot be rehabilitated even when the current density is returned to $0.5 \mathrm{C}$, indicating the sluggish kinetics and even the destruction of the electrode. Correspondingly, the charge/discharge curves of the 
$\mathrm{MC} / \mathrm{S}$ electrode at various current densities are presented in Figure 27e. At $5 \mathrm{C}$, the characteristic phased plateaus almost disappear with only a single slope can be observed, implying the poor kinetics of conversion reactions between sulfur species. With such stark contrast, the significant advantages of the $\mathrm{FeS}_{2} @ \mathrm{C}-\mathrm{S}$ electrode, in terms of structure stability, conversion kinetics, and polysulfides trapping ability, are particularly evident.
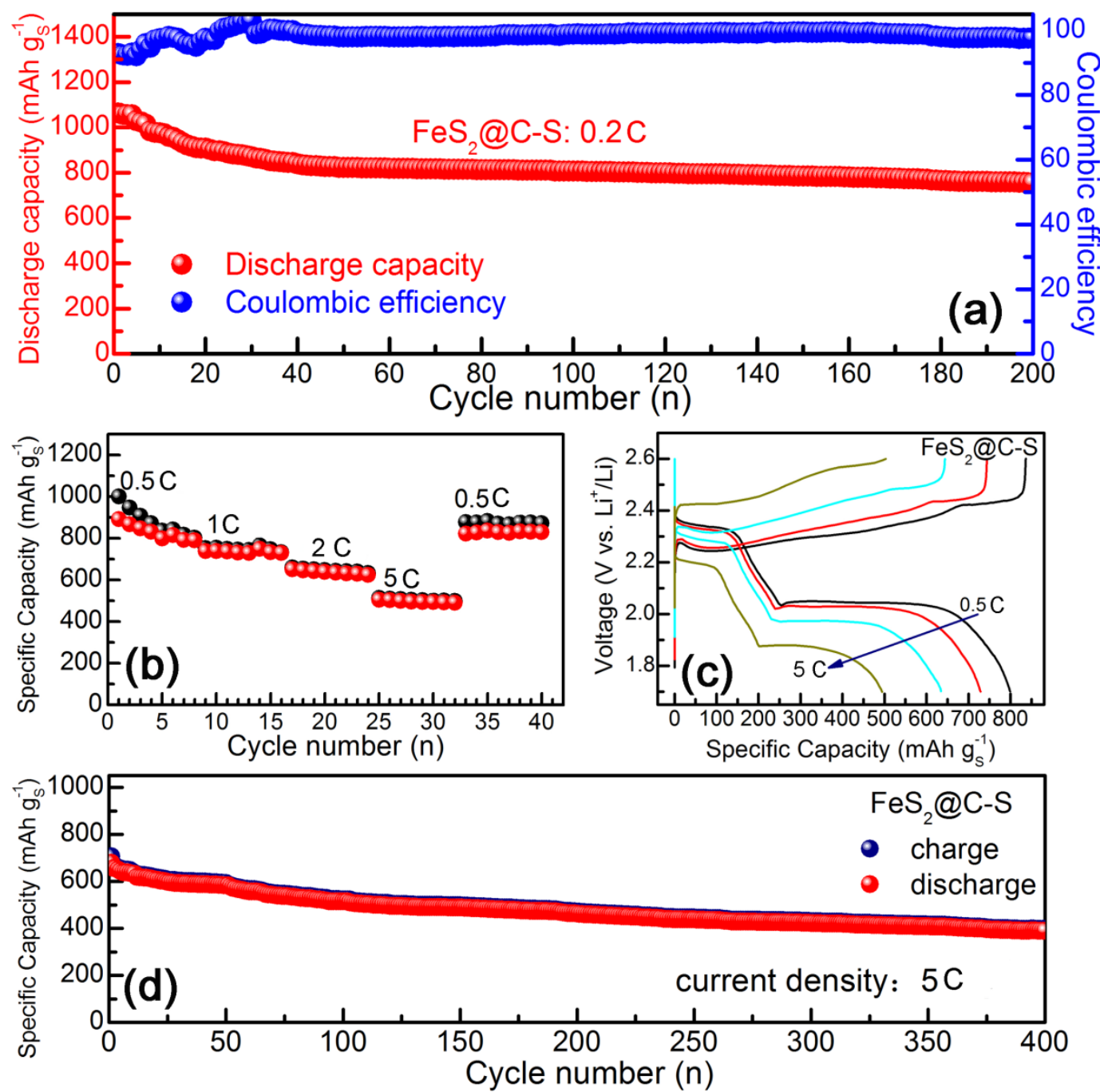

Figure 26 (a) Cycle performance of $\mathrm{FeS}_{2} @ \mathrm{C}-\mathrm{S}$ electrode at $0.2 \mathrm{C}$, (b) rate capability at various C-rates and the corresponding charge/discharge curves (c), and (d) long-term cycle performance of $\mathrm{FeS}_{2} @ \mathrm{C}-\mathrm{S}$ electrode at the current density of $5 \mathrm{C}$. 

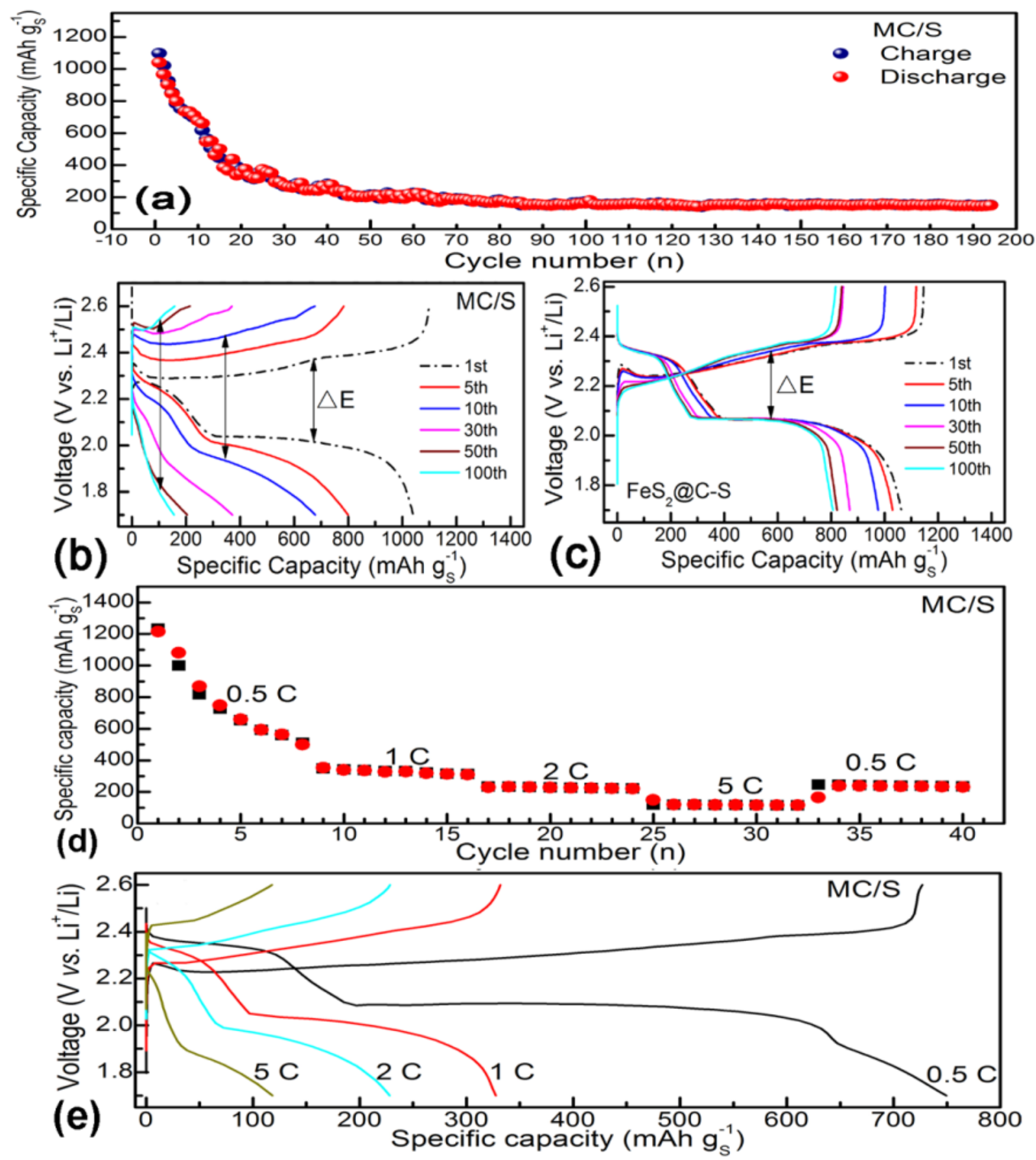

Figure 27 (a) cycle performance of MC/S at $0.2 \mathrm{C}$. Charge/discharge curves of MC/S (b) and $\mathrm{FeS}_{2} @ \mathrm{C}-\mathrm{S}$ (c) electrodes. (d) Rate capability from $0.5 \mathrm{C}$ to $5 \mathrm{C}$ with corresponding charge/discharge curves of $\mathrm{MC} / \mathrm{S}$ electrode (e). 
The excellent rate capability can be not only ascribed to the amorphous carbon cage for increasing electronic conductivity but also the hollow/void space for facilitating the rapid electrolyte ion diffusion. CV measurement was further carried out to investigate the electrode kinetics. As shown in Figure 28a, with the scan rate increasing from $0.2 \mathrm{mV} \mathrm{s}^{-1}$ to $1.0 \mathrm{mV} \mathrm{s}^{-1}$, the cathodic peaks shift to lower potential and the anodic peaks shift to higher potential due to the polarization. The diffusion coefficient of $\mathrm{Li}^{+}\left(D_{L i^{+}}\right)$can be calculated by Eqn. (1).

$$
I_{P}=2.69 \times 10^{5} A C_{L i} D_{L i}^{1 / 2} v^{1 / 2} n^{3 / 2}
$$

where $A$ is the area of the electrode, $C_{L i}$ is the concentration of electrolyte, and $n$ is the number of electrons involved in the diffusion step $(\mathrm{n}=2)$. Accordingly, Figure $28 \mathrm{~b}$ shows the square root relationship graph between peak currents $\left(I_{p}\right)$ and the scan rate $(v)$. The results show a linear relationship between peak current and potential scan rate with all $\mathrm{R}^{2}$ values closed to $100 \%$, indicating that the diffusion of $\mathrm{Li}^{+}\left(D_{\mathrm{Li}^{+}}\right)$in the electrode is the rate-determining step. The slope $\left(D_{L i^{+}}\right)$for each peak can be calculated to be $2.87 \times 10^{-8}$ (peak A), $3.76 \times 10^{-8}$ (peak B), and $1.53 \times 10^{-7} \mathrm{~cm}^{2} \mathrm{~s}^{-1}$ (peak C), respectively, which is almost $5 \sim 10$ times higher than that of reported results. ${ }^{[155]}$ The cycling stability of the $\mathrm{FeS}_{2} @ \mathrm{C}-\mathrm{S}$ electrode at higher current rates was also evaluated to demonstrate the significant advantages of the $\mathrm{FeS}_{2} @ \mathrm{C}-\mathrm{S}$ electrode with this special structure design. After 100 cycles, the capacities of $\mathrm{FeS}_{2} @ \mathrm{C}-\mathrm{S}$ electrodes can still be maintained at $650 \mathrm{mAh} \mathrm{g}^{-1}$ at $1 \mathrm{C}$ and $550 \mathrm{mAh} \mathrm{g}^{-1} 2 \mathrm{C}$, respectively (Figure 29). Even at the current density of $5 \mathrm{C}$, an exceptional reversible capacity of $\sim 400 \mathrm{mAh} \mathrm{g}^{-1}$ for the $\mathrm{FeS}_{2} @ \mathrm{C}-\mathrm{S}$ electrode can still be delivered after 400 cycles (Figure 26d), representing the significantly enhanced redox kinetics, good structural stability, and thus excellent capacity retention. In addition, the cycle performance of $\mathrm{FeS}_{2} @ \mathrm{C}$-S electrodes with a relatively higher sulfur loading was further 
evaluated to explore the potential commercial application. Figure 29c shows the cycle performance of the $\mathrm{FeS}_{2} @ \mathrm{C}-\mathrm{S}$ electrode with sulfur loading of $3.15 \mathrm{mg} \mathrm{cm}^{-2}$ at the current density of $0.2 \mathrm{C}$, which can sustain a reversible discharge capacity of $\sim 400 \mathrm{mAh} \mathrm{g}^{-1}$ after 60 cycles. However, to increase the utilization of sulfur as well as the energy/power density, future work might be conducted to promote the kinetics of electrochemical reactions without compromising the high sulfur loading by enhancing the intimate contact between the "chemical anchors" and sulfur, such as optimize the size of hollow space in the nanotube, void space between $\mathrm{FeS}_{2}$ nanoparticles, and the thickness of carbon cage. At present, the electrochemical performances of $\mathrm{FeS}_{2} @ \mathrm{C}-\mathrm{S}$ still stand out among recently reported cathodes for Li-S batteries, as compared in Table 5.
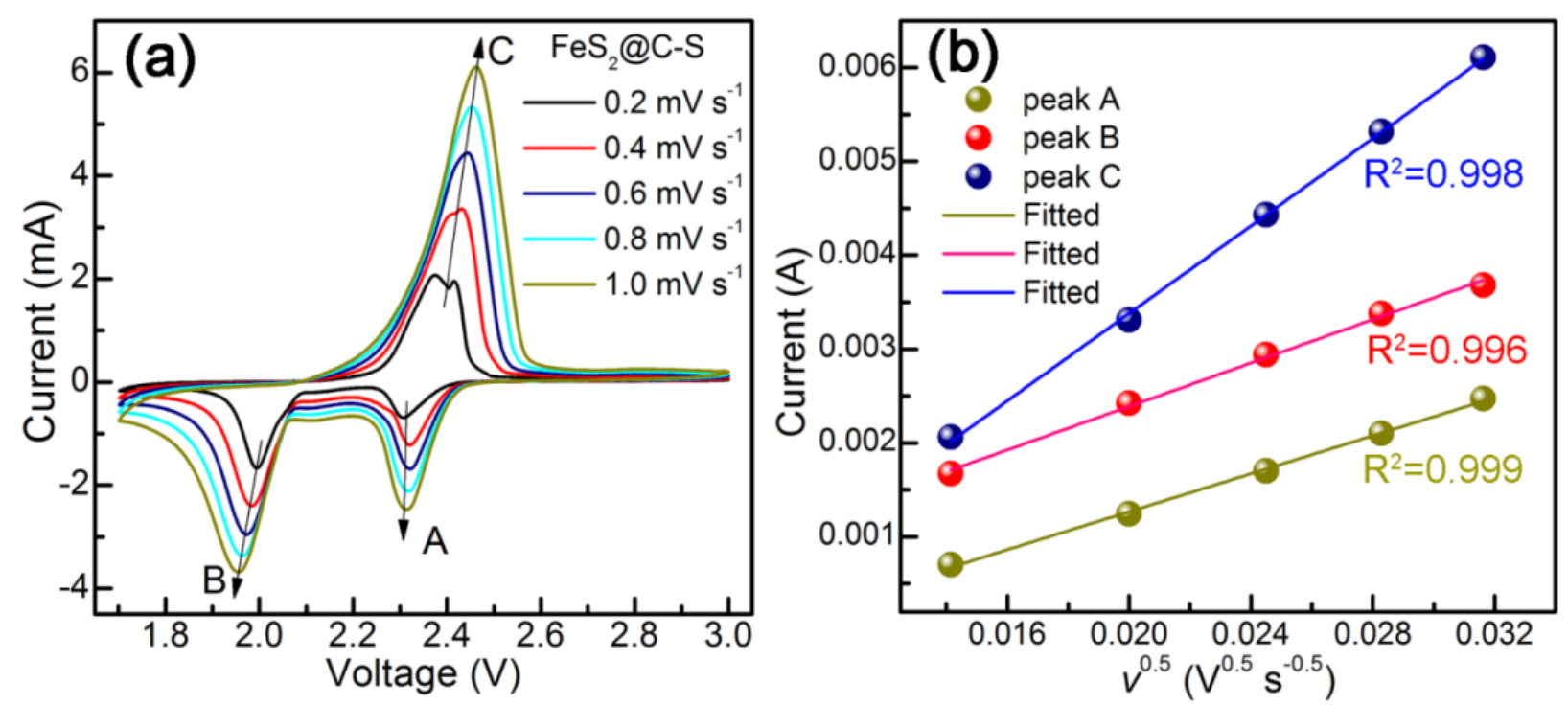

Figure 28 (a) Cyclic voltammograms of $\mathrm{FeS}_{2} @ \mathrm{C}$-S electrode at varying scan rates from 0.2 to $1.0 \mathrm{mV} \mathrm{s}^{-1}$ and (b) peak currents as a function of the square root of the scan rate. 


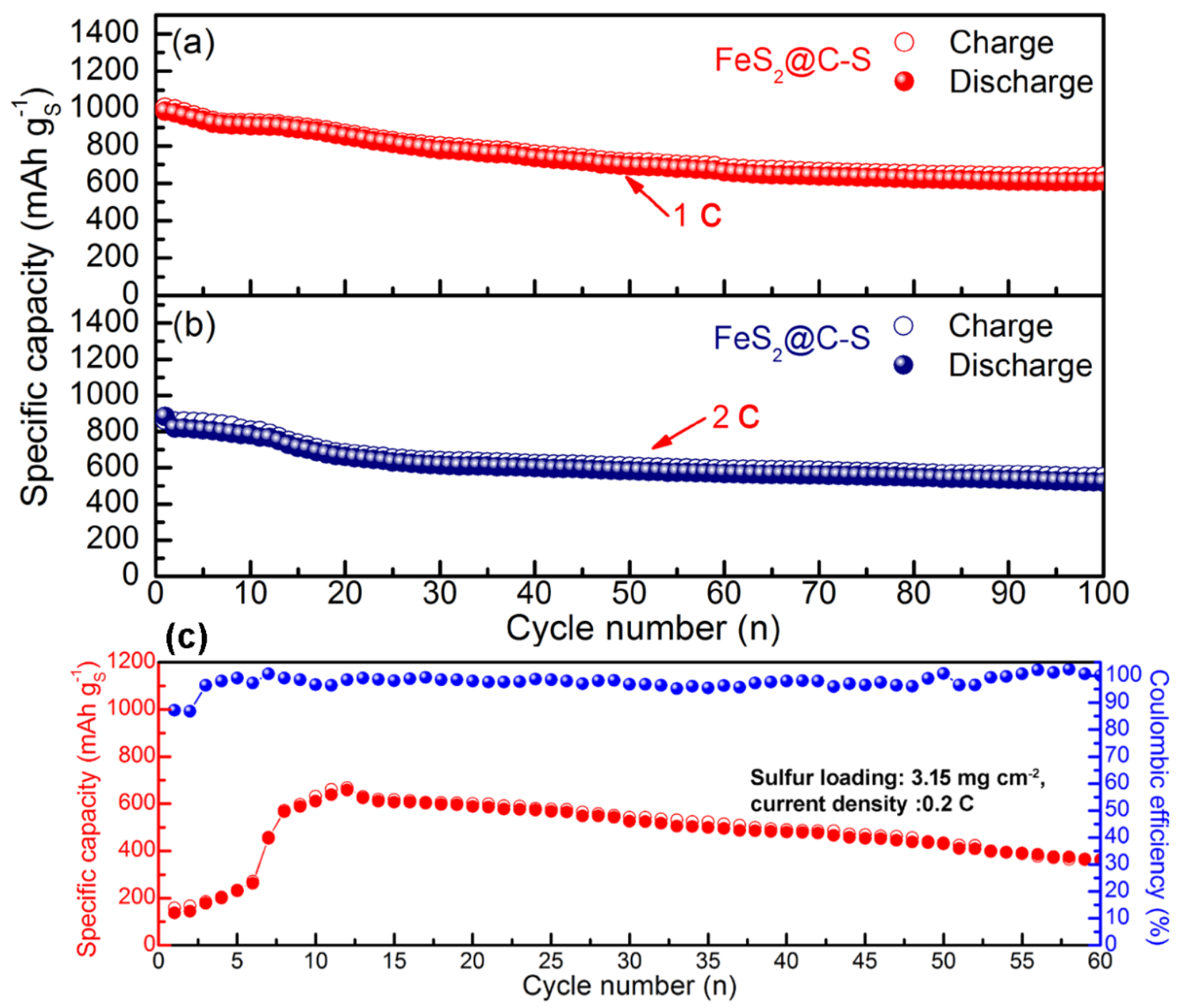

Figure 29 Cycle performance of $\mathrm{FeS}_{2} @ \mathrm{C}-\mathrm{S}$ electrodes at the current densities of $1 \mathrm{C}(\mathrm{a}), 2 \mathrm{C}$ (b) for 100 cycles. (c) Cycle performance of $\mathrm{FeS}_{2} @ \mathrm{C}-\mathrm{S}$ electrode with $\mathrm{S}$ loading of $3.15 \mathrm{mg} \mathrm{cm}^{-2}$ at the current densities of $0.2 \mathrm{C}$ for 60 cycles. 
Table 5 The performance comparison of $\mathrm{FeS}_{2} @ \mathrm{C}-\mathrm{S}$ with reported cathodes.

\begin{tabular}{|c|c|c|c|c|c|}
\hline Cathodic materials & $\begin{array}{l}\text { Sulfur loading } \\
\left(\mathrm{mg} \mathrm{cm}^{-2}\right)\end{array}$ & C-Rate & $\begin{array}{l}\text { Capacity } \\
\left(\mathrm{mAh} \mathrm{g}^{-1}\right)\end{array}$ & $\begin{array}{l}\text { Coulombic } \\
\text { efficiency }\end{array}$ & Reference \\
\hline \multirow{3}{*}{$\mathbf{F e S}_{2} @ \mathbf{C - S}$} & $\sim 1.5$ & $0.2 \mathrm{C}$ & $770(200)$ & $97.6 \%$ & \multirow{3}{*}{ This work } \\
\hline & $\sim 1.5$ & $5 \mathrm{C}$ & $400(450)$ & $98.1 \%$ & \\
\hline & 3.15 & $0.1 \mathrm{C}$ & $\sim 400(60)$ & $99.1 \%$ & \\
\hline S/NiS@C-HS & 1.0 & $0.2 \mathrm{C}$ & $718(200)$ & $\sim 97 \%$ & [119] \\
\hline $\begin{array}{l}\text { RGO-g-poly(S-r- } \\
\text { IDBI) }\end{array}$ & $1.0-1.5$ & $0.2 \mathrm{C}$ & $714(100)$ & $\sim 100 \%$ & {$[155]$} \\
\hline \multirow{2}{*}{ S/G-DBD } & \multirow{2}{*}{1.6} & $0.2 \mathrm{C}$ & $754(200)$ & $\sim 100 \%$ & \multirow{2}{*}[156]{} \\
\hline & & $0.5 \mathrm{C}$ & $618(500)$ & NA & \\
\hline $\mathrm{Ni} / \mathrm{Ni}_{3} \mathrm{~S}_{2} / \mathrm{S}$ & 4.0 & ${ }_{2}^{1} \mathrm{~mA} \mathrm{~cm}^{-}$ & $654(80)$ & $\sim 92 \%$ & {$[157]$} \\
\hline $\mathrm{Li}_{2} \mathrm{~S} / \mathrm{CMK} 3 / \mathrm{C}$ & 1.3 & $0.2 \mathrm{C}$ & $705(150)$ & $\sim 98 \%$ & {$[158]$} \\
\hline S@NiCo-DH@RC & 1.5 & $0.2 \mathrm{C}$ & $972(250)$ & $99.8 \%$ & [159] \\
\hline $\mathrm{MgB}_{2}-\mathrm{S60}$ & 2.0 & $0.5 \mathrm{C}$ & $\sim 800(200)$ & $98.4 \%$ & {$[160]$} \\
\hline \multirow{2}{*}{ S/PPA } & \multirow{2}{*}{1.5} & $0.5 \mathrm{C}$ & $763(100)$ & $97 \%$ & \multirow{2}{*}{ [161] } \\
\hline & & $1.5 \mathrm{C}$ & $430(400)$ & $95 \%$ & \\
\hline \multirow{2}{*}{ S-NC@Al+NC@PP } & \multirow{2}{*}{$1.6-2.0$} & $0.2 \mathrm{C}$ & $679(100)$ & $\mathrm{NA}$ & \multirow{2}{*}[162]{} \\
\hline & & $2 \mathrm{C}$ & $267(500)$ & NA & \\
\hline \multirow{2}{*}{$\mathrm{Li}_{2} \mathrm{~S}-\mathrm{CoSe}_{2} / \mathrm{G}$} & 1.0 & $4 \mathrm{C}$ & $503(500)$ & $\sim 93 \%$ & \multirow{2}{*}[163]{} \\
\hline & 4.35 & $0.5 \mathrm{C}$ & $832(100)$ & NA & \\
\hline PCC-S & 1.4 & $0.125 \mathrm{C}$ & $570(100)$ & $\sim 100 \%$ & {$[164]$} \\
\hline \multirow{2}{*}{$\mathrm{S} \| \mathrm{rGO} @ \mathrm{MoS}_{2}$} & \multirow{2}{*}{$1.8-2.0$} & $0.2 \mathrm{C}$ & $671(200)$ & NA & \multirow{2}{*}[165]{} \\
\hline & & $1 \mathrm{C}$ & $368(500)$ & $98 \%$ & \\
\hline \multirow{2}{*}{ S/CNT/PrNP } & 1.1 & $0.5 \mathrm{C}$ & $695(200)$ & $95 \%$ & \multirow{2}{*}[166]{} \\
\hline & 3.8 & $0.2 \mathrm{C}$ & $685(200)$ & $\mathrm{NA}$ & \\
\hline S-APP & $2-3$ & $0.5 \mathrm{C}$ & $640(400)$ & $99 \%$ & [167] \\
\hline TiN-C65 & 0.6 & $0.2 \mathrm{C}$ & $680(100)$ & $\sim 98 \%$ & {$[168]$} \\
\hline OCNT/S & 1.5 & $0.5 \mathrm{C}$ & $535(100)$ & NA & [169] \\
\hline S/C with NCM & 4.0 & $0.5 \mathrm{C}$ & $\sim 550(100)$ & $\sim 98 \%$ & {$[170]$} \\
\hline HMO-S & 2.8 & $0.25 \mathrm{C}$ & $792(100)$ & NA & {$[171]$} \\
\hline $\mathrm{S} @ \mathrm{Ni} / \mathrm{Fe}$ LDH & 2.3 & $0.2 \mathrm{C}$ & $725(200)$ & $99 \%$ & {$[172]$} \\
\hline Fe-N-C/G@PP & 1.0 & $0.5 \mathrm{C}$ & $602(500)$ & $\sim 100 \%$ & {$[173]$} \\
\hline
\end{tabular}


The good cycle stability and excellent rate capability of the $\mathrm{FeS}_{2} @ \mathrm{C}-\mathrm{S}$ electrode finally confirm the design advantages of this type of electrode. The unique structure with void space between $\mathrm{FeS}_{2}$ nanoparticles as the major sulfur chamber, which are further caged in the hollow carbon shell, can not only facilitate fast electrons $/ \mathrm{Li}^{+}$transportation, accommodate the volume change of electrode, but also provide a physical barrier to prevent the diffusion of polysulfides. In addition, the elaborately built "chemical anchors", including C-S bonding (during the sulfurization process), polar interaction and redox catalyst $\left(\mathrm{FeS}_{2}\right)$, provide a comprehensive synergistic effect on the polysulfides chemisorption and conversion to impede the "shuttle effects", which is due to the much higher binding energy between the "chemical anchors" and polysulfides and the critical role of $\mathrm{FeS}_{2}$ in catalyzing the polysulfides conversion reactions. ${ }^{[142,}$ ${ }^{174]}$ The long-chain polysulfides are soluble in the electrolyte, which is the main reason for the shuttle effect. The $\mathrm{FeS}_{2}$ nanoparticles enabled fast kinetics of polysulfides conversion and therefore minimized the chance for the polysulfides migration to the anode side, and thus can alleviate the shuttle effect. The polysulfides-trapping ability can be also visualized by the morphology change of the electrode film and the corresponding EIS evolution curves in Figure 30a-f. Initially, both of the as-prepared MC/S (Figure 30a) and FeS $@$ @C-S (Figure 30d) electrode films show uniformly distributed particles without any large bulk sulfur on the surface. After 100 cycles, the MC/S electrode film (Figure 30b) becomes much more blurred due to the insulating nature of sulfur species diffused and accumulated on the electrode surface. Such accumulated process results in the gradually increased charge transfer resistance with cycling, as shown in Figure 30c. However, the morphology of the cycled FeS ${ }_{2} @ \mathrm{C}-\mathrm{S}$ electrode film (Figure 30e) doesn't change too much compared with the fresh one (Figure 30d), indicating the effective polysulfides trapping capability. As also demonstrated in Figure 30f, the charge transfer 
resistances keep very stable and even slightly decrease during the 100 cycles. The EDX mapping of the $\mathrm{MC} / \mathrm{S}$ electrode after cycle (Figure 31) clearly reveals the seriously aggregated sulfur species on the surface. Contrastingly, for the $\mathrm{FeS}_{2} @ \mathrm{C}-\mathrm{S}$ electrode, $\mathrm{C}, \mathrm{Fe}, \mathrm{S}$, and $\mathrm{O}$ elements are still homogeneously dispersed in the carbon matrix (Figure 32). To further illustrate the chemisorption mechanism, XPS analysis of $\mathrm{FeS}_{2} @ \mathrm{C}$-S electrode film after 100 cycles (discharged to $2.2 \mathrm{~V}$ ) was conducted as shown in Figure 30g-i. Besides the carbonate species formed on the electrode surface $(\mathrm{O}=\mathrm{C}-\mathrm{O}$ groups in Figure 30g), the peak corresponding to C-S bonding is shifted to higher binding energy by $0.4 \mathrm{eV}$ and can still be remained even after 100 cycles, indicating the strong interaction between polysulfides and S-doped carbon host. ${ }^{[175]}$ During the discharge process, long-chain sulfur will be gradually lithiated with the "-S-S-" bonds broken and combined with $\mathrm{Li}^{+}$to form short polysulfides. However, it was reported that "C-S" bonding is strong enough and cannot be further broken. ${ }^{[43]}$ This means the density of electron clouds around the sulfur atom will be increased when the long " $\mathrm{C}-\left(\mathrm{S}_{\mathrm{x}}\right)-$ " chain lithiated to form "C-S-Li" and short polysulfides, as the electronegativity of sulfur is much higher than that of $\mathrm{Li}$, and sulfur can attract more electrons from adjacent $\mathrm{Li}$ and $\mathrm{C}$. The slight charge accumulation on sulfur atom yields the stronger "C-S" and "S-Li" bonds due to the higher electronegativity of sulfur. Therefore, the "C-S" peak in the C $1 s$ spectrum shifts to higher binding energy due to the possibly decreased electron clouds of carbon atom. The $S 2 p$ spectrum in Figure 30h shows three dominant peaks as well as polythionate and thiosulfate groups. The peaks centered at $162.8 \mathrm{eV}$, $161.7 \mathrm{eV}$, and $160.5 \mathrm{eV}$ should correspond to the residual bridging $\left(S_{B}^{0}\right)$, terminal $\left(S_{T}^{-1}\right)$ sulfur species, and sulfides, respectively. All these peaks show a little shift to lower binding energy compared with the results of fresh electrode film (Figure 23f), which also provides evidence for the possible chemical interaction between polysulfides species and those "chemical anchors". ${ }^{[159,}$ 
${ }^{175 b, 176]}$ Due to the less electronegativity $\mathrm{Li}$ and $\mathrm{Fe}$ atom than $\mathrm{S}$ atom, the formation of short polysulfides ("Li-S" bonds) during the lithiation process and chemical interaction between polysulfides and $\mathrm{FeS}_{2}\left(\mathrm{Li}-\mathrm{S} . . . \mathrm{FeS}_{2}\right)$ through "Fe-S" bonds will induce the increased density of electron clouds of $\mathrm{S}$ atoms, leading to the shift of corresponding peaks to the lower binding energy. Li $1 s$ spectrum is also displayed in Figure 8i to reveal the possible interaction of LiPSs with the host material. It can be seen that peaks at 58.2, 55.8, 54.2, 53.3 eV correspond to RO-Li, $\mathrm{LiF} / \mathrm{Li}_{2} \mathrm{O}, \mathrm{Li}_{2} \mathrm{CO}_{3}, \mathrm{LiOH}$, respectively, resulting from the electrolyte decomposition. In addition, the peak of $\mathrm{Li}_{2} \mathrm{~S}$ centered at $52.0 \mathrm{eV}$ can be also identified. This binding energy is much lower than that reported ones and pure $\mathrm{Li}_{2} \mathrm{~S},{ }^{[46,152 \mathrm{c}, 177]}$ which also suggesting the possible interaction between polysulfides and host material. ${ }^{[178]}$ 

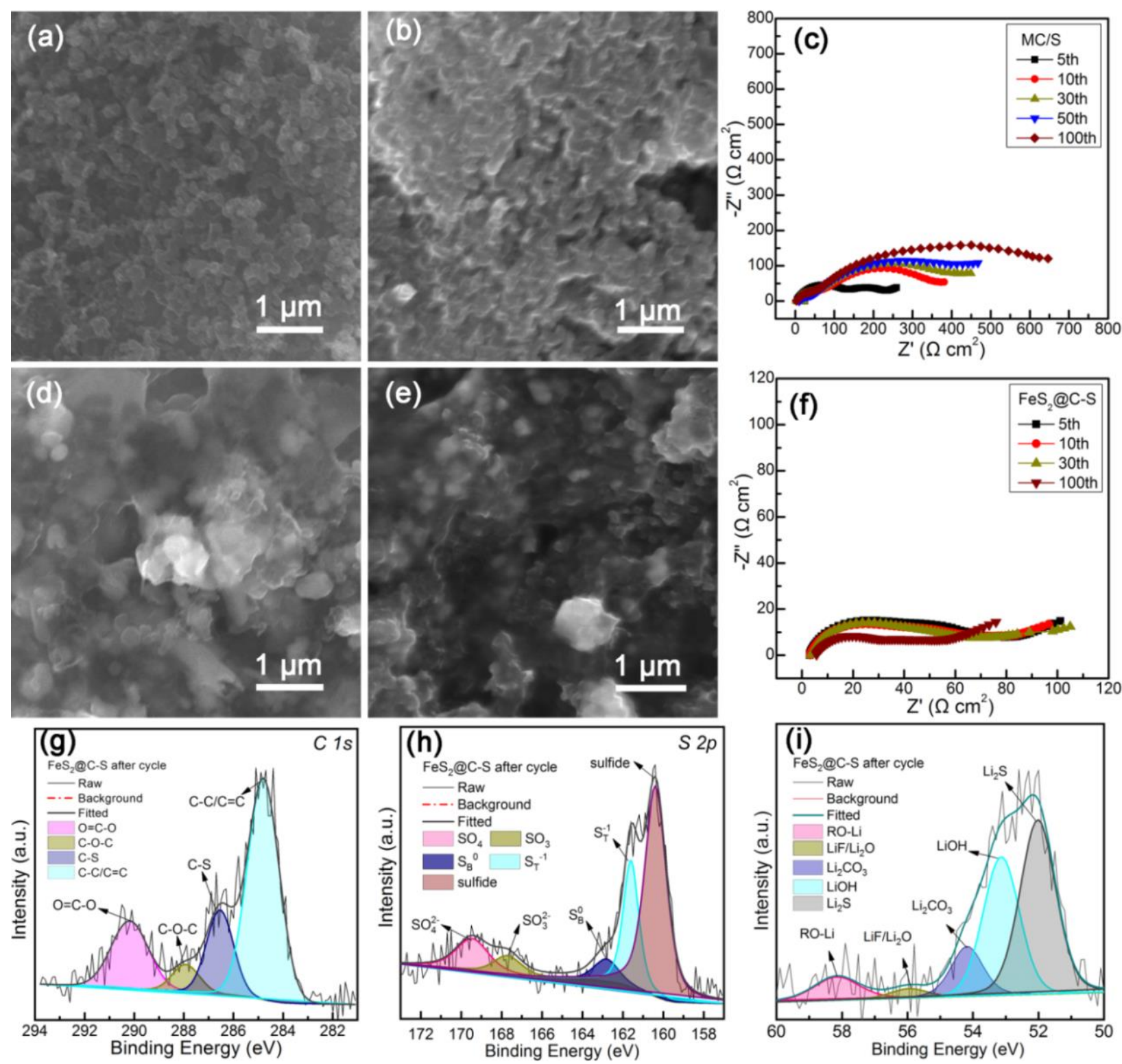

Figure 30 SEM images of fresh (a) and cycled (b) MC/S electrode film with corresponding EIS Nyquist curves at different cycles (c). SEM images of fresh (d) and cycled (e) FeS $\mathrm{F}_{2} @ \mathrm{C}-\mathrm{S}$ electrode film with corresponding EIS Nyquist curves at different cycles (f), and high-resolution XPS spectra of (g) C $1 s$, (h) S $2 p$, (i) Li $1 s$ peaks of cycled FeS $\mathrm{F}_{2} @ \mathrm{C}-\mathrm{S}$ electrode film. 


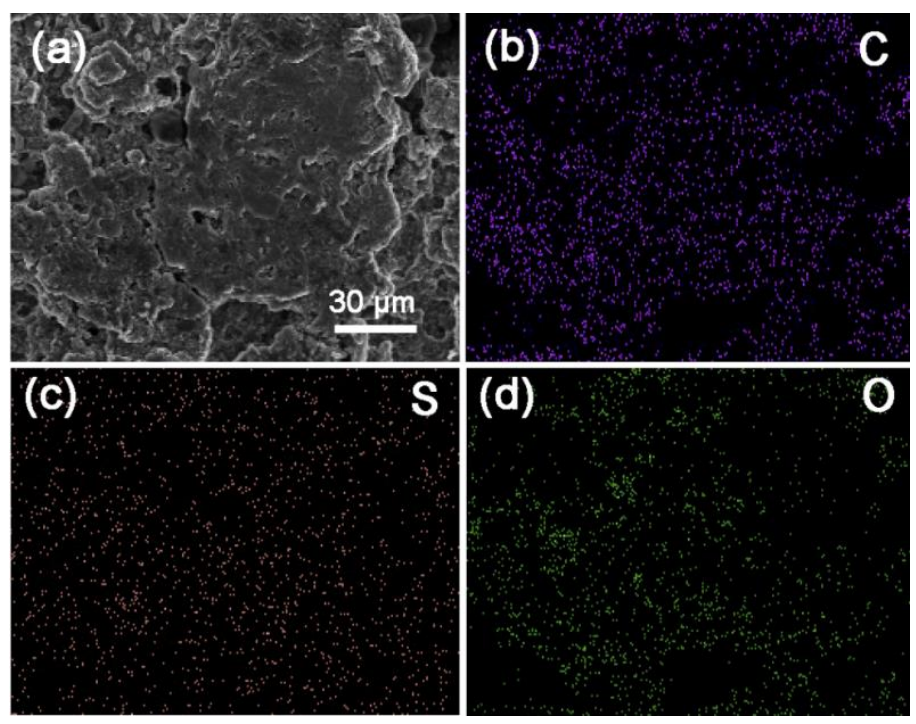

Figure 31 (a) SEM images of cycled MC/S electrode film and corresponding elemental maps of $\mathrm{C}(\mathrm{b}), \mathrm{S}(\mathrm{c}), \mathrm{O}(\mathrm{d})$.
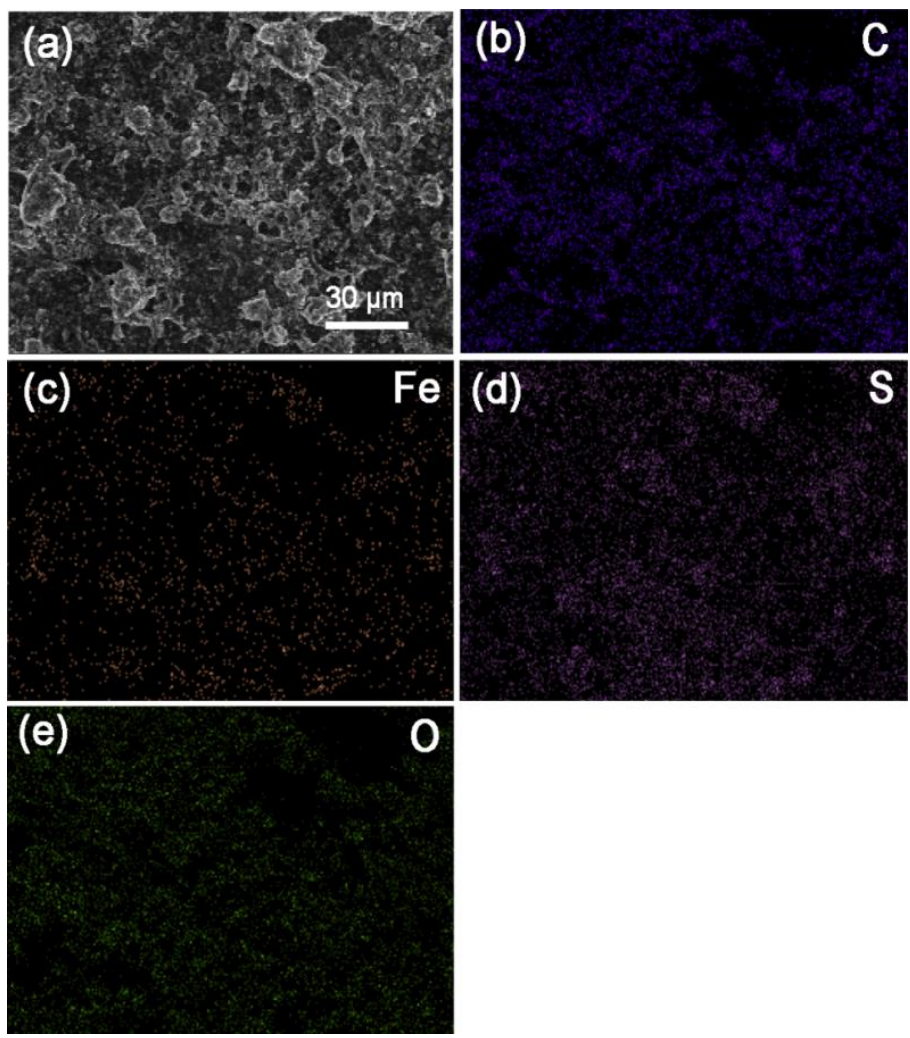

Figure 32 (a) SEM images of cycled $\mathrm{FeS}_{2} @ \mathrm{C}-\mathrm{S}$ electrode film and corresponding elemental maps of C (b), Fe (c), S (d), and O (e). 


\subsection{Conclusion}

In summary, we facilely develop a sulfur host with nanosized $\mathrm{FeS}_{2}$ particles caged in a hollow carbon shell for Li-S batteries application. This elaborately designed composite (FeS $\left.\mathrm{F}_{2} @ \mathrm{C}-\mathrm{S}\right)$ can significantly alleviate the polysulfides "shuttle effects" and deliver enhanced electrochemical performance due to the integrated different functional units as follows:

(1) The refined $\mathrm{FeS}_{2}$ nanoparticles, which intimately contact with active sulfur, can act as a robust redox mediator to facilitate the kinetics of LiPSs-involved redox reaction;

(2) The sufficient "chemical anchors", such as $\mathrm{C}-\mathrm{O}, \mathrm{C}-\mathrm{S}$, and $\mathrm{FeS}_{2}$, provide rich adsorption sites to immobilize polysulfides through the polar-polar interaction;

(3) The hollow carbon cage can promote both the electrons and Li ion transportation, resulting in the lower charge transfer resistances and high $\mathrm{Li}^{+}$flux for electrode kinetics, thus enables the excellent rate capability; besides, the carbon shell with hollow structure can act as a physical barrier to prevent the polysulfides diffusion and accommodate the volume change to keep the stability of electrode;

This multi-functional composite, therefore, could achieve outstanding performance, in terms of cycle stability, rate capability, and Coulombic efficiency. This work reports a facile design for sulfur host materials and provides promising guidance to develop Li-S batteries with high energy, high rate, and long cycle life. 


\section{Chapter 5: Programmed Design of a Li-S Battery Cathode by}

\section{Integrating Functional Units}

\subsection{Introduction}

To further increasing the sulfur loading, a bioinspired electrode structure design is proposed to construct self-supported cathodes. This design of the cathode electrode integrates Ni foam framework, host carbon, carbon nanofibers, and $\mathrm{Ni}_{3} \mathrm{~S}_{2}$ with active sulfur $\left(\mathrm{S} / \mathrm{CNF}-\mathrm{HC}-\mathrm{Ni}_{3} \mathrm{~S}_{2}\right)$ for high-performance Li-S batteries through a programmed fabrication approach (Figure 33).

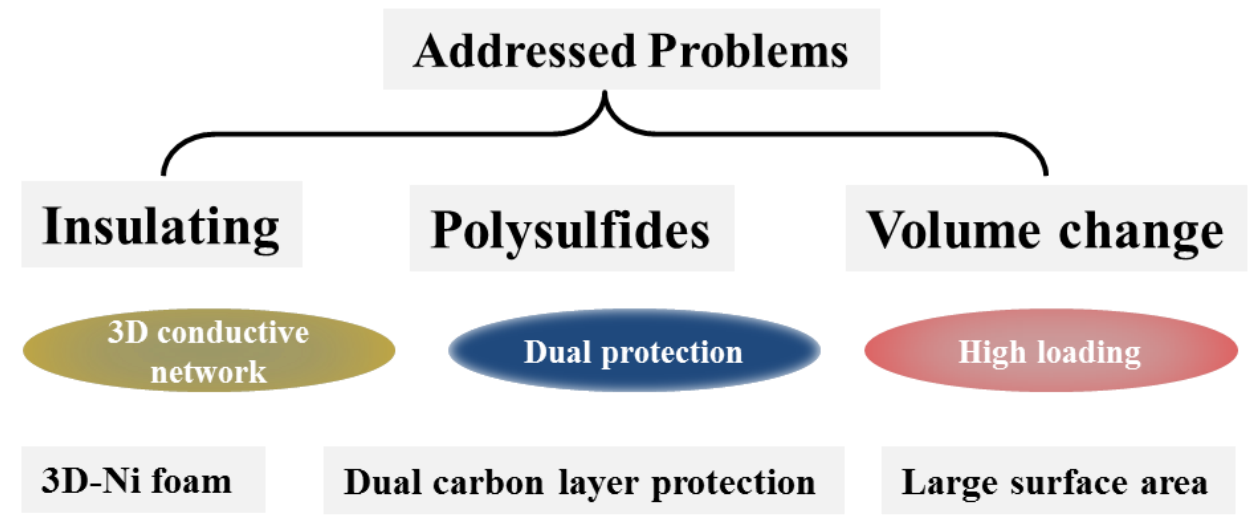

Figure 33 Design route for high sulfur loading electrode development by integrating 3D conductive Ni foam network, dual functional carbon layer with large surface area.

As illustrated in Figure 34a, the architecture of such cathode mimics the structure of giardia lamblia, a parasitic microorganism. Two kinds of carbon layers are in situ grown on Ni foam framework, consisting of S-doped host carbon and carbon nanofiber forest, corresponding to the "sucker" and "flagella", respectively. Considerable $\mathrm{Ni}_{3} \mathrm{~S}_{2}$ nanoparticles are uniformly distributed in the carbon matrix, similar to the "nucleus". The HC layer serves as the primary reservoir for the loading of sulfur. As the giardia lamblia shows strong adhesion ability to the surface of the infected hosts, this electrode with a similar structure design is expected to have an affinity 
towards LiPSs species. In this work, such an elaborately designed cathode possesses cooperative interfaces of "lithiophilic" S-doped carbon and "sulfiphilic" $\mathrm{Ni}_{3} \mathrm{~S}_{2}$ (Figure 34b). The $\mathrm{Ni}_{3} \mathrm{~S}_{2}$ particles have strong chemical adsorption affinity to polysulfide and high electrocatalytic activity for facilitating the LiPSs-involved redox reactions. The HC layer can enable a relatively high loading of sulfur and the partially sulfurized CNF layer can act as a barrier/functional layer to prevent the diffusion of LiPSs and facilitate the transport of Li ions and electrons, both of which can also accommodate the volume changes. Overall, this hierarchical electrode design integrates multiple building blocks with specialized roles into an ensemble to show a synergistic effect, providing a firm and effective three-dimensional (3D) conductive network and cooperative interfaces to minimize the shuttle effect by increasing the density of adsorption sites, adsorption capability, electron/ion transfer and catalytic redox kinetics for the sulfur species during the discharge-charge process. As a result, the S/CNF-HC-Ni $\mathrm{S}_{2}$ cathode exhibits a stable reversible capacity of $\sim 850 \mathrm{mAh} \mathrm{g}^{-1}$ after 100 cycles at a current density of $0.2 \mathrm{C}$, excellent rate capability, and superior cycle durability $\left(620 \mathrm{mAh} \mathrm{g}^{-1}\right.$ after 300 cycles at $2 \mathrm{C}$ and $400 \mathrm{mAh} \mathrm{g}^{-1}$ after 500 cycles at $5 \mathrm{C}$ ). This work offers a programmed design strategy by integrating hierarchical functional units to develop high-performance cathodes for Li-S batteries. 


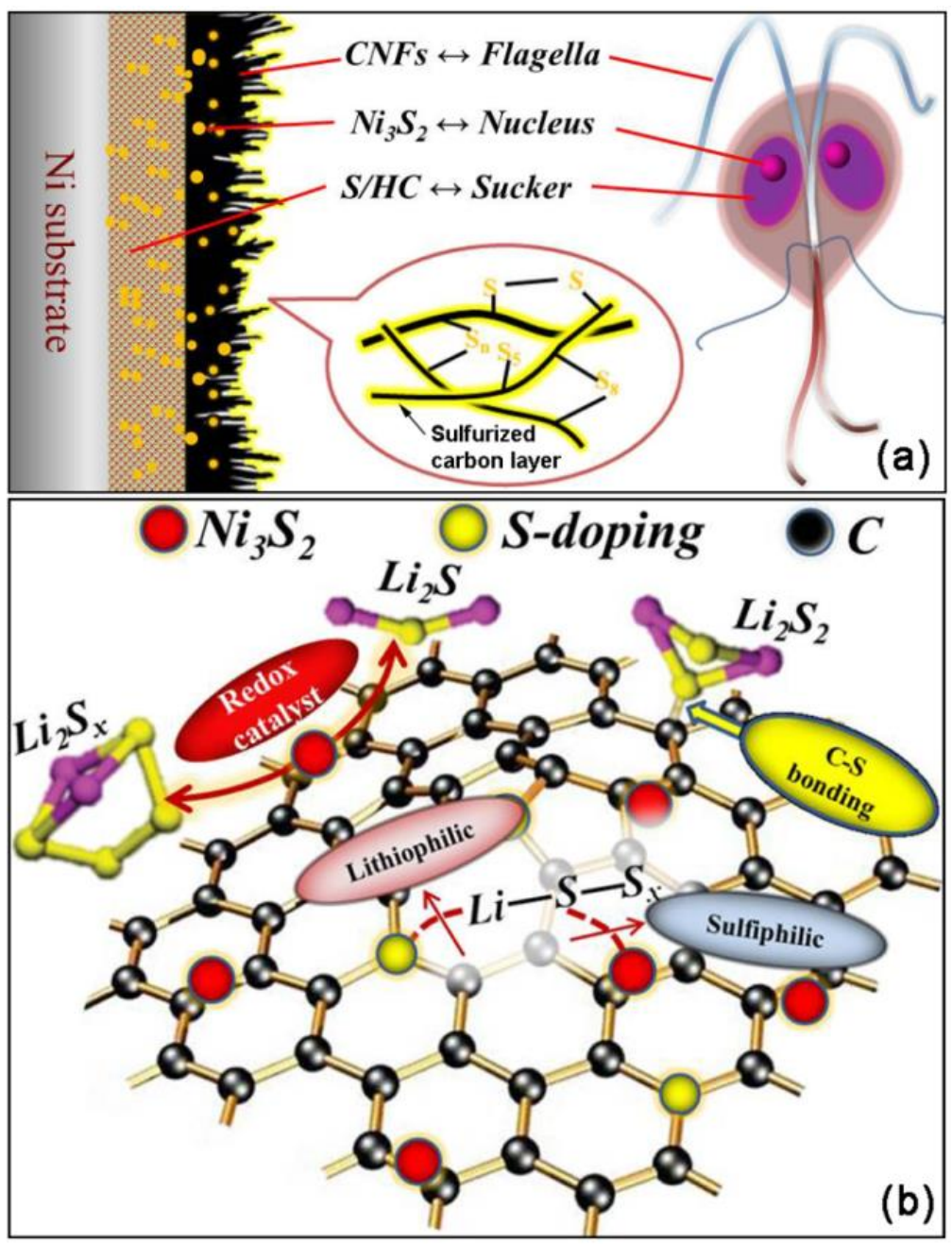

Figure 34 Schematic illustration of (a) the flagellate-like S/CNF-HC-Ni $\mathrm{S}_{2}$ cathode and giardia lamblia. (b) The mechanism of cooperative lithiophilic and sulfiphilic interfaces of S-doped carbon and $\mathrm{Ni}_{3} \mathrm{~S}_{2}$ for enhanced adsorption and electrocatalytic conversion of lithium polysulfides.

\subsection{Synthesis of S/CNF-HC-Ni $\mathrm{S}_{2}$ electrode and related control samples.}

Surface modification of Ni foam: a piece of Ni form was firstly punched into circular discs with a diameter of $\sim 10 \mathrm{~mm}$ and then pressed under $1500 \mathrm{lb}$ pressure for 2 min to keep the mechanical strength during the following process. Five $\mathrm{Ni}$ form discs were subsequently immersed into the $80 \mathrm{~mL}$ deionized (DI) water dissolving $0.45 \mathrm{~g} \mathrm{FeSO}_{4} \cdot 7 \mathrm{H}_{2} \mathrm{O}, 0.2 \mathrm{~g}$ urea, and $0.016 \mathrm{~g}$ sodium lauryl sulfate (SDS). The transparent yellow solution with Ni discs was then 
transferred into a Teflon-lined stainless steel autoclave with a capacity of $100 \mathrm{~mL}$ for hydrothermal treatment at $100{ }^{\circ} \mathrm{C}$ for $12 \mathrm{~h}$. The obtained $\mathrm{Ni}$ foam discs were collected and washed with DI water and absolute ethanol several times and then dried under vacuum at $80{ }^{\circ} \mathrm{C}$. In this way, the $\mathrm{Ni}$ foams were coated with $\mathrm{NiFe}_{2} \mathrm{O}_{4}$ layers, as confirmed in Figure 35.

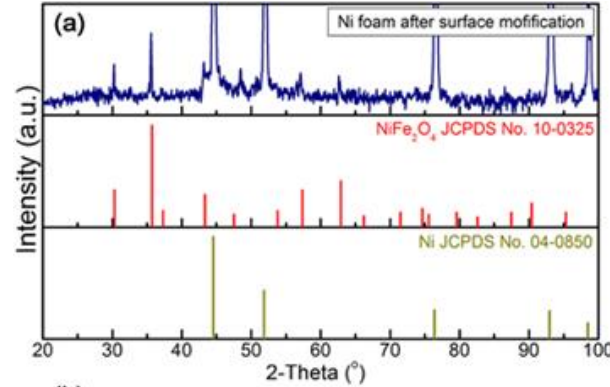

(b)
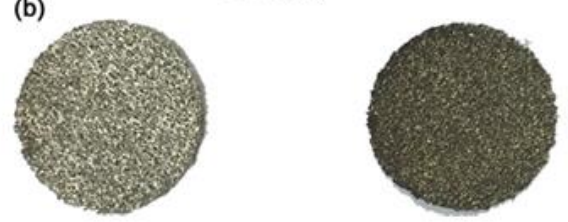

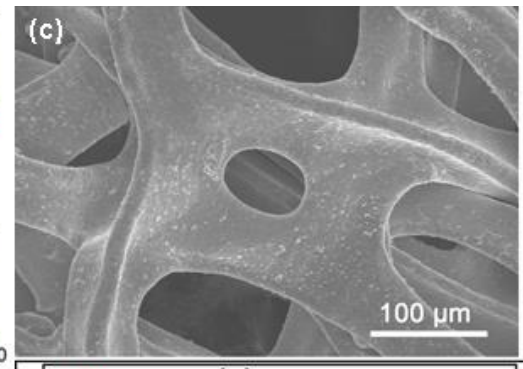

(e) $\mathrm{NiFe}_{2} \mathrm{O}_{4}$ modified $\mathrm{Ni}$ foam
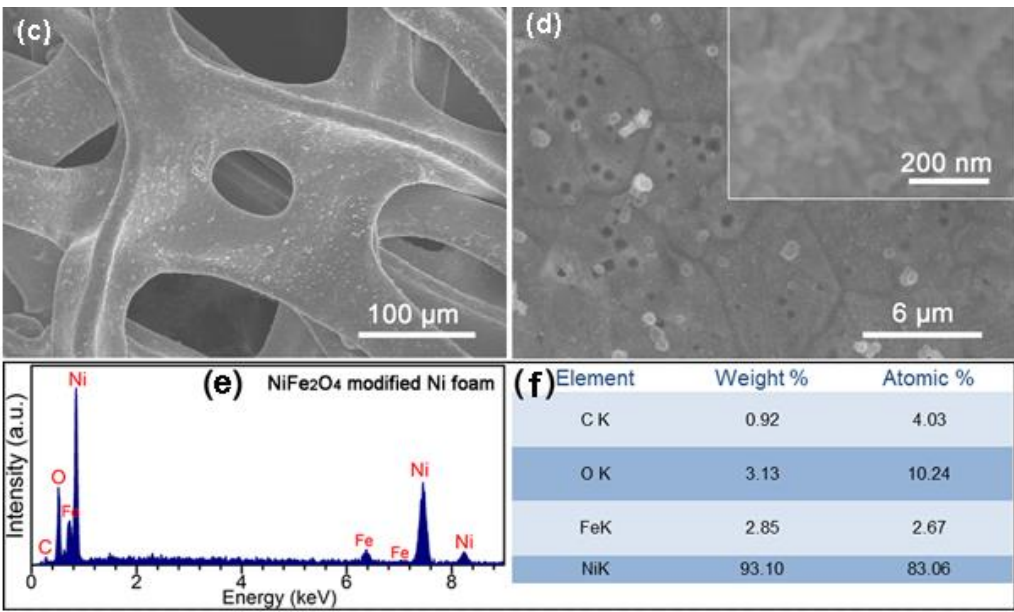

Atomic \%

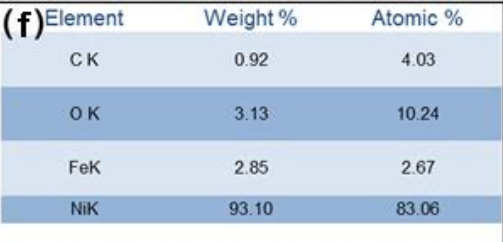

Figure 35 (a) XRD pattern of the $\mathrm{NiFe}_{2} \mathrm{O}_{4}$-modified $\mathrm{Ni}$ foam after the hydrothermal process and standard XRD cards of $\mathrm{NiFe}_{2} \mathrm{O}_{4}$ and Ni. (b) Photographs of $\mathrm{Ni}$ foam discs before (left) and after (right) the hydrothermal surface modification treatment. SEM images (c, d), EDX spectrum (e), and quantitative analysis (f) of $\mathrm{NiFe}_{2} \mathrm{O}_{4}$-modified $\mathrm{Ni}$ foam.

Synthesis of $3 D$ conductive $\mathrm{S} / \mathrm{CNF}-\mathrm{HC}-\mathrm{Ni}_{3} \mathrm{~S}_{2}$ electrode: the $\mathrm{NiFe}_{2} \mathrm{O}_{4}$ modified $\mathrm{Ni}$ foam discs arranged on a Ni foam slab shelving on a combustion boat (Figure 36) was put into a quartz tube for chemical vapor deposition (CVD) process. The toluene was used as the carbon source and carried by $5 \% \mathrm{H}_{2} / \mathrm{Ar}$ at a flow rate of $0.1 \mathrm{~L} \mathrm{~min}^{-1}$. The reaction system was heated to $800{ }^{\circ} \mathrm{C}$ at a ramping rate of $5{ }^{\circ} \mathrm{C} \mathrm{min}^{-1}$ and maintained at this temperature for $3 \mathrm{~h}$ to enable the growth of carbon nanofibers and host carbon layers (CNF-HC) over pre-modified Ni foam discs. After the CVD process, each $\mathrm{Ni}$ foam disc was mixed with $c a .50 \mathrm{mg}$ sulfur powder and sealed in a separate vial under the protection of $\mathrm{Ar}$ gas and then heated at $300{ }^{\circ} \mathrm{C}$ for $1 \mathrm{~h}$ to create the 
chemical anchors mainly composed of $\mathrm{Ni}_{3} \mathrm{~S}_{2}$ and sulfurized carbon. Then a certain amount of sulfur powder $(\sim 10 \mathrm{mg})$ was mixed with each $\mathrm{Ni}$ foam disc, which was heated at $155{ }^{\circ} \mathrm{C}$ in $\mathrm{Ar}$ for $12 \mathrm{~h}$ to infiltrate sulfur into host carbon layers. Finally, the disc was rapidly rinsed by $\mathrm{CS}_{2}$ to remove possible bulk sulfur on the surface, and then the monolithic $\mathrm{S} / \mathrm{CNF}-\mathrm{HC}-\mathrm{Ni}_{3} \mathrm{~S}_{2}$ electrode was obtained. The sulfur loading for each disc was controlled to $\sim 2 \mathrm{mg} \mathrm{cm}^{-2}$ unless stated otherwise.

Synthesis of $3 D$ conductive S/CNF-HC electrode: the control sample, S/CNF-HC, was synthesized by a similar method except for the absence of thermal sulfurization treatment at 300 ${ }^{\circ} \mathrm{C}$. After the CVD growth of CNF-HC layers on Ni foam discs, a certain amount of sulfur powder $(\sim 10 \mathrm{mg})$ was mixed with each disc, which was heated at $155{ }^{\circ} \mathrm{C}$ in $\mathrm{Ar}$ for $12 \mathrm{~h}$ to infiltrate sulfur into host carbon layers. Finally, the disc was rapidly rinsed by $\mathrm{CS}_{2}$ to remove possible bulk sulfur on the surface. The S/CNF-HC electrode has a similar architecture yet without the $\mathrm{Ni}_{3} \mathrm{~S}_{2}$ component.

Synthesis of $3 D$ conductive S/CMP electrode: as a comparison, pristine Ni foam discs without hydrothermal pretreatment were also used in a similar CVD process to enable the growth of carbon microspheres (CMPs) layers over their surfaces. In the absence of the formation of chemical anchors at $300{ }^{\circ} \mathrm{C}$, the same amount of sulfur powder was mixed with CMP-modified $\mathrm{Ni}$ foam disc, which was heated at $155{ }^{\circ} \mathrm{C}$ in $\mathrm{Ar}$ for $12 \mathrm{~h}$ to infiltrate sulfur into CMP layers. Finally, the disc was also rinsed by $\mathrm{CS}_{2}$ and the self-supported S/CMP electrode was obtained.

Synthesis of $\mathrm{Ni}_{3} \mathrm{~S}_{2} / \mathrm{Ni}$ electrode: the $\mathrm{Ni}_{3} \mathrm{~S}_{2} / \mathrm{Ni}$ electrode was prepared by directly mixing the $\mathrm{Ni}$ foam discs and sulfur powder for the thermal sulfurization at $300{ }^{\circ} \mathrm{C}$ for $1 \mathrm{~h}$ in $\mathrm{Ar}$. 


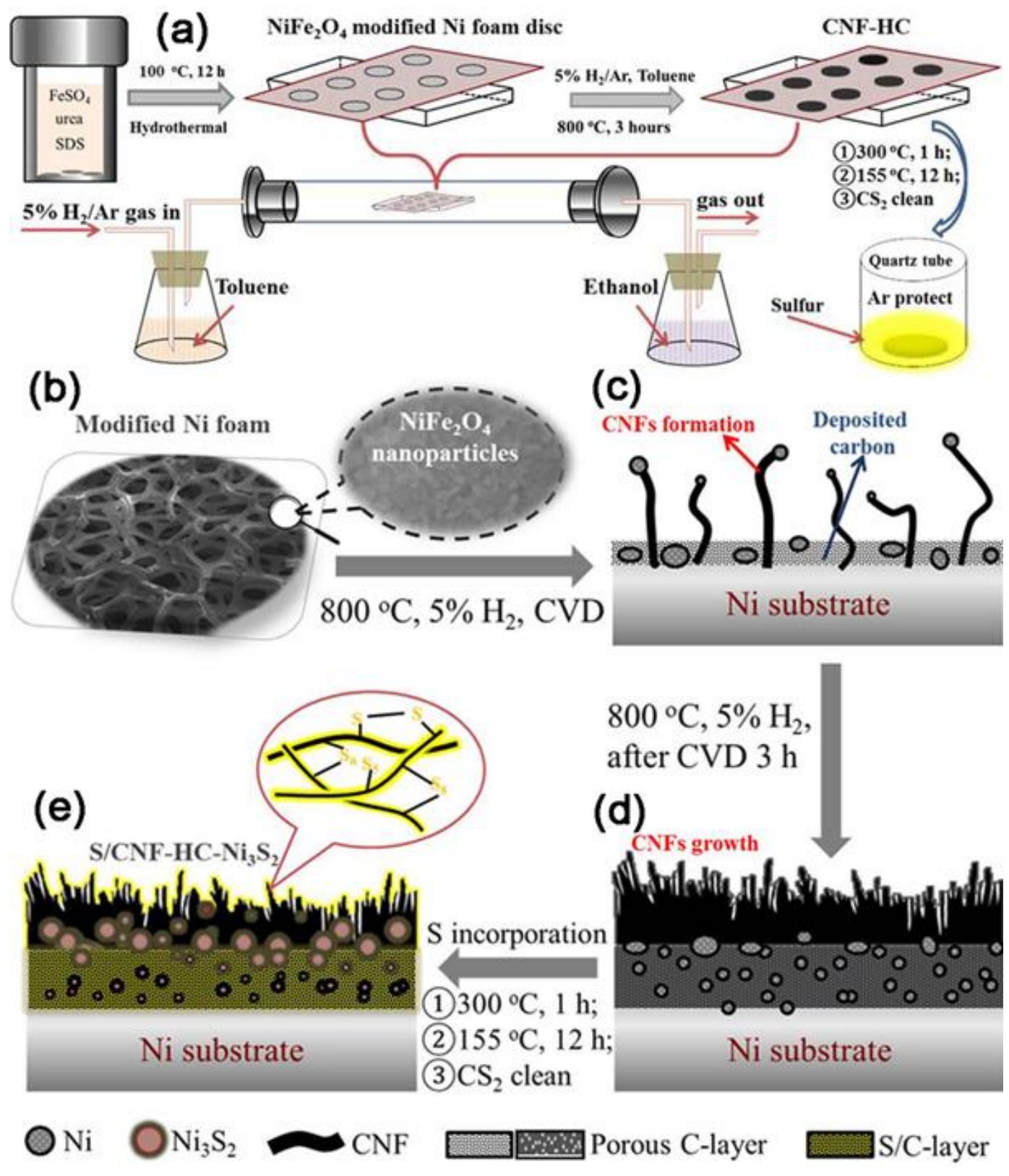

Figure 36 Schematic illustrations of the whole programmed fabrication process for synthesizing $\mathrm{S} / \mathrm{CNF}-\mathrm{HC}-\mathrm{Ni}_{3} \mathrm{~S}_{2}$.

\subsection{Coin cells assemble and electrochemical tests}

Li-S cells assembly and measurement: the coin cells were assembled using either selfsupported S/CNF-HC-Ni $\mathrm{S}_{2}, \mathrm{~S} / \mathrm{CNF}-\mathrm{HC}$, or S/CMP discs as the working electrode, lithium metal foil (MTI Corporation) as the counter electrode, and porous polypropylene (Celgard 2400) as a 
separator. The liquid electrolyte was 1M lithium bis(trfluoromethanesulfonyl) imide (LiTFSI) and $1 \% \mathrm{LiNO}_{3}$ dissolved in dioxolane (DOL) and dimethoxyethane (DME) $(1: 1 \mathrm{v} / \mathrm{v})$. The coin cells (CR2032) were fabricated in an argon-filled glove box (moisture and oxygen levels less than 1ppm). The electrochemical performance of the cells was tested by Arbin BT2143 32CH with the voltage range between 1.7 and $3.0 \mathrm{~V}$ vs. $\mathrm{Li}^{+} / \mathrm{Li}$. The rate performance of the corresponding cells was tested at various current densities from $0.2 \mathrm{C}$ to $5 \mathrm{C}\left(1 \mathrm{C}=1000 \mathrm{~mA} \mathrm{~g}^{-1}\right)$. The cyclic voltammetry (CV) measurements were conducted with the electrochemical workstation (Gamry Interface 5000E) at a scan rate of $0.1 \mathrm{mV} \mathrm{s}^{-1}$ within a voltage range of 1.7$3.0 \mathrm{~V}$.

Symmetrical cells assembly and measurement: the electrodes for symmetrical cells were fabricated without the presence of elemental sulfur. Either CNF-HC or CNF-HC-Ni $\mathrm{S}_{2}$ discs were used as identical working and counter electrodes. $30 \mu \mathrm{L}$ electrolytes containing $1 \mathrm{M} \mathrm{Li}_{2} \mathrm{~S}_{6}$ dissolved in DOL/DME (1:1, v/v) was injected into each coin cell. The dark brown $\operatorname{Li}_{2} \mathrm{~S}_{6}$ electrolyte was prepared by mixing $\mathrm{Li}_{2} \mathrm{~S}$ ad $\mathrm{S}$ into the solvent at a molar ratio of $1: 5$ under stirring at $60{ }^{\circ} \mathrm{C}$ for 12 hours in Ar. $\mathrm{CV}$ measurements of the symmetrical cells were performed at scan rate of $10 \mathrm{mV} \mathrm{s}^{-1}$ within a voltage range from -1.2 to $1.2 \mathrm{~V}$. Electrochemical impedance spectroscopy (EIS) measurements were performed with the Gamry Interface 5000E at open circuit potential with sinusoidal potential excitation of $5 \mathrm{mV}$ amplitude. The frequency range was from $1 \mathrm{MHz}$ to $0.1 \mathrm{~Hz}$.

\subsection{Physical and chemical structure characterizations}

The programmed fabrication process of $\mathrm{S} / \mathrm{CNF}-\mathrm{HC}-\mathrm{Ni}_{3} \mathrm{~S}_{2}$ electrodes includes the hydrothermal surface pre-treatment of $\mathrm{Ni}$ foam, chemical vapor deposition (CVD) for growth of host carbon and carbon nanofiber forest and stepwise thermal treatment for $\mathrm{Ni}_{3} \mathrm{~S}_{2}$ and sulfur 
incorporation followed by $\mathrm{CS}_{2}$ rinsing to remove bulk $\mathrm{S}$ residues. The average $\mathrm{S}$ mass ratio is estimated to $8 \mathrm{wt}$. \% by thermal gravimetric analysis. The magnification of the XRD pattern of cleaned S/CNF-HC-Ni $\mathrm{S}_{2}$ (Figure 37a) can unambiguously identify the existence of many minor peaks of the rhombohedral $\mathrm{Ni}_{3} \mathrm{~S}_{2}$ phase (JCPDS No. 44-1418) and a broad peak centered at $26.4^{\circ}$ arising from the graphitic carbon matrices with sulfur. One sharp peak at $2 \theta \approx 22^{\circ}$ for $\mathrm{Ni}_{3} \mathrm{~S}_{2}$ somehow disappears, which is probably due to the broad hump and ascending background from the graphitic carbon overlapping with it. Few sharp peaks of crystalline sulfur can be observed, indicating that sulfur was well dispersed in the carbon layer of the cathode. The morphologies of $\mathrm{Ni}$ foam substrate and $\mathrm{S} / \mathrm{CNF}-\mathrm{HC}-\mathrm{Ni}_{3} \mathrm{~S}_{2}$ cathode were characterized by SEM at different stages of the preparation process. After the CVD process, the smooth surface of Ni foam is coated with numerous carbon nanofibers (Figure 38). Then after stepwise thermal treatment for $\mathrm{Ni}_{3} \mathrm{~S}_{2}$ and sulfur incorporation, the morphology of CNFs has little change except for the slight coarsening (Figure 37b). Few agglomerations of bulk sulfur particles can be observed suggesting the homogeneous dispersion and loading of sulfur within the S/CNF-HC-Ni $\mathrm{S}_{2}$, consistent with the XRD results. The energy dispersive X-ray (EDX) elemental mapping of the ligament surface of $\mathrm{S} / \mathrm{CNF}-\mathrm{HC}-\mathrm{Ni}_{3} \mathrm{~S}_{2}$ demonstrates that $\mathrm{Ni}$ and $\mathrm{S}$ elements are homogeneously dispersed in the carbon matrix without notable segregation (Figure 39). To clearly reveal the hierarchical architecture of $\mathrm{Ni}$ foam subjected to CVD treatment and cleaned S/CNF-HC-Ni $\mathrm{S}_{2}$, their crosssectional SEM images are shown in Figure 37c and d, respectively. Three layers can be observed distinctly after the CVD process, including the top layer of CNF forest, interlayer of HC with void space, and $\mathrm{Ni}$ substrate layer for the $\mathrm{Ni}$ foam. S/CNF-HC-Ni $\mathrm{S}_{2}$ has a similar hierarchical structure, while the $\mathrm{HC}$ interlayer is filled with sulfur, and in-situ formed $\mathrm{Ni}_{3} \mathrm{~S}_{2}$ nanoparticles are embedded in the whole matrix. The cross-sectional EDX line scan profile and spectrum (Figure 
37-f) of S/CNF-HC-Ni ${ }_{3} \mathrm{~S}_{2}$ corroborate that sulfur is primarily loaded in the $\mathrm{HC}$ reservoir and $\mathrm{Ni}_{3} \mathrm{~S}_{2}$ is dispersed in CNF and HC matrix. This is the embodiment of the design that we conceived in Figure 34a.
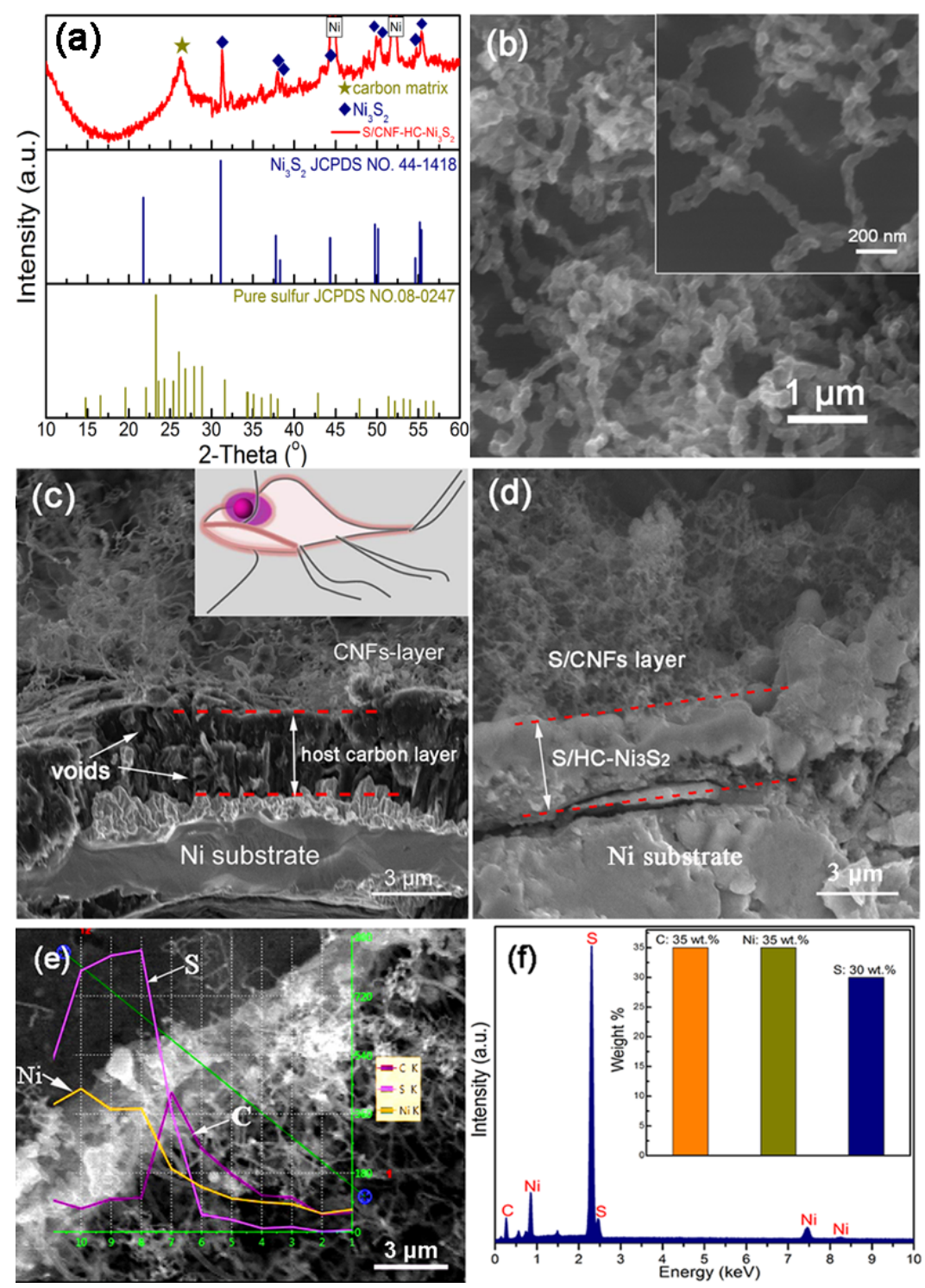

Figure 37 (a) The magnified XRD pattern of S/CNF-HC-Ni $\mathrm{S}_{2}$ and standard XRD cards of $\mathrm{Ni}_{3} \mathrm{~S}_{2}$ and S. SEM images of the top view of (b) S/CNF-HC-Ni $\mathrm{S}_{2}$ electrode. Cross-sectional SEM images of (c) Ni foam after the CVD process and (d) S/CNF-HC-Ni $\mathrm{S}_{2}$ electrode. Inset of (c) is a lateral view of the giardia lamblia structure. (e) EDX line scan profile and (f) EDX spectrum of $\mathrm{S} / \mathrm{CNF}-\mathrm{HC}-\mathrm{Ni}_{3} \mathrm{~S}_{2}$ with the corresponding elemental quantification in the inset. 


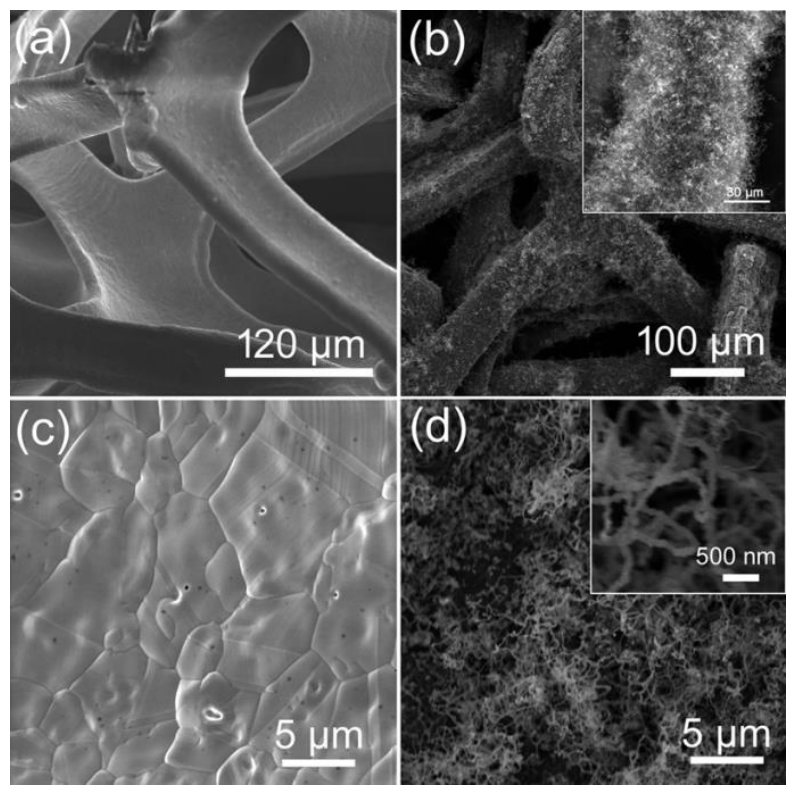

Figure 38 SEM images of fresh Ni foam at low (a) and high (c) magnification, and the SEM images for hydrothermally treated $\mathrm{Ni}$ foam after CVD process $(b, \mathrm{~d})$ with the flourishing growth of carbon nanofibers (CNFs) on the surface.

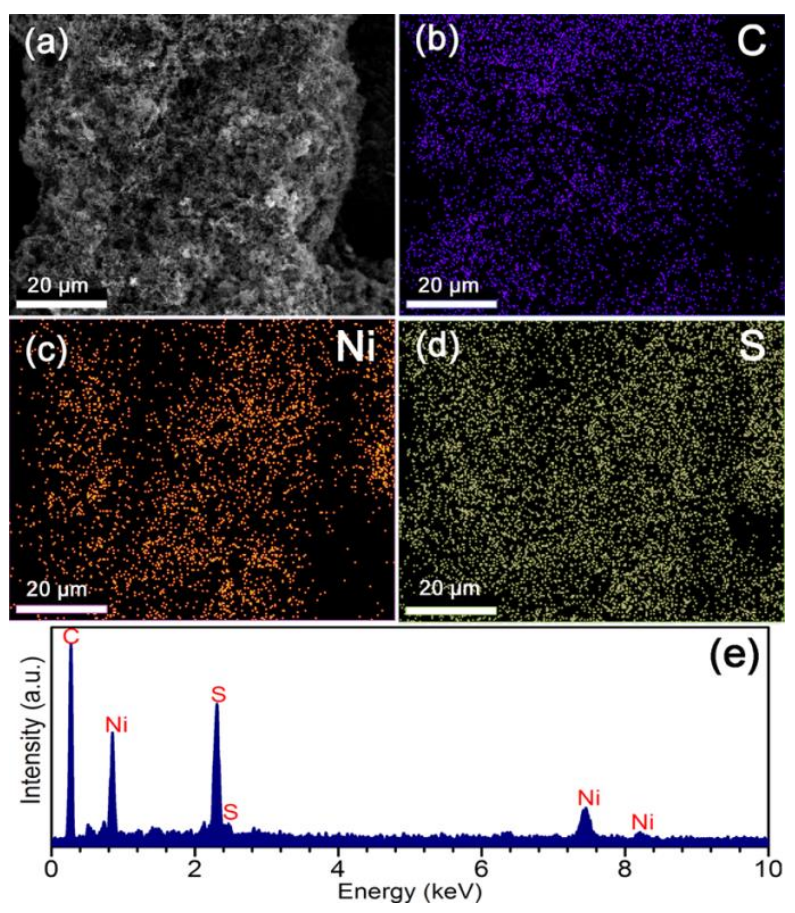

Figure $39 \mathrm{SEM}$ images of pristine $\mathrm{S} / \mathrm{CNF}-\mathrm{HC}-\mathrm{Ni}_{3} \mathrm{~S}_{2}$ and corresponding elemental maps of $\mathrm{C}(\mathrm{b})$, $\mathrm{Ni}(\mathrm{c})$, and S (d) and its EDX spectrum. 
The chemical interactions within S/CNF-HC-Ni $\mathrm{S}_{2}$ especially associated with the chemical adsorption towards LiPSs were further investigated by FTIR, Raman, and XPS. In the FTIR spectrum (Figure 40a), the typical peaks at 1575 and $1665 \mathrm{~cm}^{-1}$ can be ascribed to the $\mathrm{C}=\mathrm{C}$ stretching vibrations originating from the graphite planar. ${ }^{[145]}$ The peak at $1728 \mathrm{~cm}^{-1}$ is assigned to symmetric stretching vibrations of $-\mathrm{COOH}$ groups on the pyrolysis carbon. ${ }^{[145 b, 146]}$ The characteristic peaks at 1330 and $1450 \mathrm{~cm}^{-1}$ can be due to the $\mathrm{H}-\mathrm{C}$ stretching modes of $\mathrm{H}-\mathrm{C}=\mathrm{O}$ in carboxyl groups. ${ }^{[146]}$ Other oxygen-containing and $\mathrm{CH}$ groups can be also identified at the peaks of $1240(\mathrm{C}-\mathrm{O}-\mathrm{C}), 1052(\mathrm{C}-\mathrm{OH})$, and $740 \mathrm{~cm}^{-1}(\mathrm{C}-\mathrm{H})$, respectively. ${ }^{[179]}$ These oxygen-containing functional groups can act as active sites bonding with the short sulfur chains, resulting in a peak at $1028 \mathrm{~cm}^{-1}$ corresponding to the O-S vibration. ${ }^{[96]}$ The characteristic peaks of C-S bonds are located at 670 and $955 \mathrm{~cm}^{-1},{ }^{[180]}$ while the peak of Ni-S bond is at $1100 \mathrm{~cm}^{-1} \cdot{ }^{[181]}$ In the Raman spectrum (Figure 40b), two prominent peaks at 1376 and $1585 \mathrm{~cm}^{-1}$ corresponding to the D (disordered carbon) and $\mathrm{G}$ (graphitic carbon) bands are well-documented in the previous literature. ${ }^{[147]}$. A sharp peak at $\sim 1440 \mathrm{~cm}^{-1}$ is likely assigned to $\mathrm{C}-\mathrm{H}\left[\delta\left(\mathrm{CH}_{2}\right)\right]$ deformation caused by the methyl group from the residual toluene. ${ }^{[182]}$ Additionally, two small humps centered at the peaks of 792 and $938 \mathrm{~cm}^{-1}$ correspond to C-S and S-S vibrations, respectively. ${ }^{[44,183]}$ A hump region from $100 \mathrm{~cm}^{-1}$ to $500 \mathrm{~cm}^{-1}$ indicates complicated vibration modes, where the peaks at 140 , 243 , and $406 \mathrm{~cm}^{-1}$, are assigned to the vibrational modes of nickel sulfides. ${ }^{[184]}$ In addition, the characteristic peaks of C-S and S-S can be identified at 308 and $468 \mathrm{~cm}^{-1}$, respectively, indicating that sufficient active anchor sites for chemical adsorption towards polysulfides have been successfully created in this integrated electrode during the sulfurization process. ${ }^{[4,, 83]}$ The C 1s XPS spectrum can be deconvoluted into four peaks (Figure 40c). The typical peak at 284.8 $\mathrm{eV}$ and $286.9 \mathrm{eV}$ can be ascribed to $s p^{2}$ hybridized carbon and C-O species, respectively. The 
peak at $285.6 \mathrm{eV}$ corresponding to C-S bonds proves the covalent bonding between sulfur and carbon matrices in the composite. ${ }^{[94,149]}$ A shoulder peak at $283.7 \mathrm{eV}$ corresponds to the Ni-C bond resulting from the $\mathrm{Ni}_{3} \mathrm{C}$ formed during the CVD process. ${ }^{[147 \mathrm{~b}, 185]}$ The $\mathrm{S} 2 p$ spectrum (Figure 40d) demonstrates a broad peak centered at $168.4 \mathrm{eV}$, which is assigned to the sulfate resulting from the adventitious oxidation of the surface during the sample transfer. ${ }^{[102,147 e, 151]}$ As sulfur and $\mathrm{Ni}_{3} \mathrm{~S}_{2}$ are both included in the electrode, there should be two $2 p_{3 / 2} / 2 p_{1 / 2}$ doublets in the S $2 p$ spectrum. The peaks at $164.6 \mathrm{eV}$ and $163.6 \mathrm{eV}$ can be attributed to the spin-orbit coupling, but the binding energy of the $\mathrm{S} 2 p_{3 / 2}$ peak $(163.6 \mathrm{eV})$ is lower than that of elemental sulfur (164.0 $\mathrm{eV})$, reconfirming the chemical bonding sulfur atoms with carbon matrix $(\mathrm{C}-\mathrm{S}) .{ }^{[42,}$ 94, 186] The other two peaks at $163.5 \mathrm{eV}$ and $162.4 \mathrm{eV}$ should be reasonably attributed to $\mathrm{Ni}_{3} \mathrm{~S}_{2}$, which also coincides with the reported literature. ${ }^{[152]}$ The $\mathrm{Ni} 2 p_{3 / 2}$ and $2 p_{1 / 2}$ peaks at 856.1 and $873.7 \mathrm{eV}$ are coupled with their satellite peaks at 861.7 and $879.7 \mathrm{eV}$ (Figure 40e), respectively, consistent with those of reported $\mathrm{Ni}_{3} \mathrm{~S}_{2} \cdot{ }^{[157,}$, 187] This confirms the existence of $\mathrm{Ni}_{3} \mathrm{~S}_{2}$ in S/CNF-HC-Ni $\mathrm{S}_{2}$ along with the XRD results. Figure 40f shows the XPS peak of the O $1 s$ core level of pristine $\mathrm{S} / \mathrm{CNF}-\mathrm{HC}-\mathrm{Ni}_{3} \mathrm{~S}_{2}$, which is deconvoluted into two peaks. The peak at $532.6 \mathrm{eV}$ can be due to the $\mathrm{C}=\mathrm{O}$ groups in the aromatic ring; while the other peak at $531.3 \mathrm{eV}$ is due to the $\mathrm{C}-\mathrm{O}$ bonds. This $\mathrm{C}-\mathrm{O}$ binding energy is slightly lower than the reported values, indicating that the $\mathrm{O}$ atoms are possibly sulfurized to form the C-O-S bonds as functional groups. ${ }^{[95,153]}$ Collectively, these morphological, structural and spectroscopic characterization results substantiate that selfsupported S/CNF-HC-Ni $\mathrm{S}_{2}$ electrode was obtained on the basis of our elaborate design with hierarchical architecture integrating Ni foam framework, carbon host reservoir, carbon nanofiber forest and $\mathrm{Ni}_{3} \mathrm{~S}_{2}$ with active sulfur. 

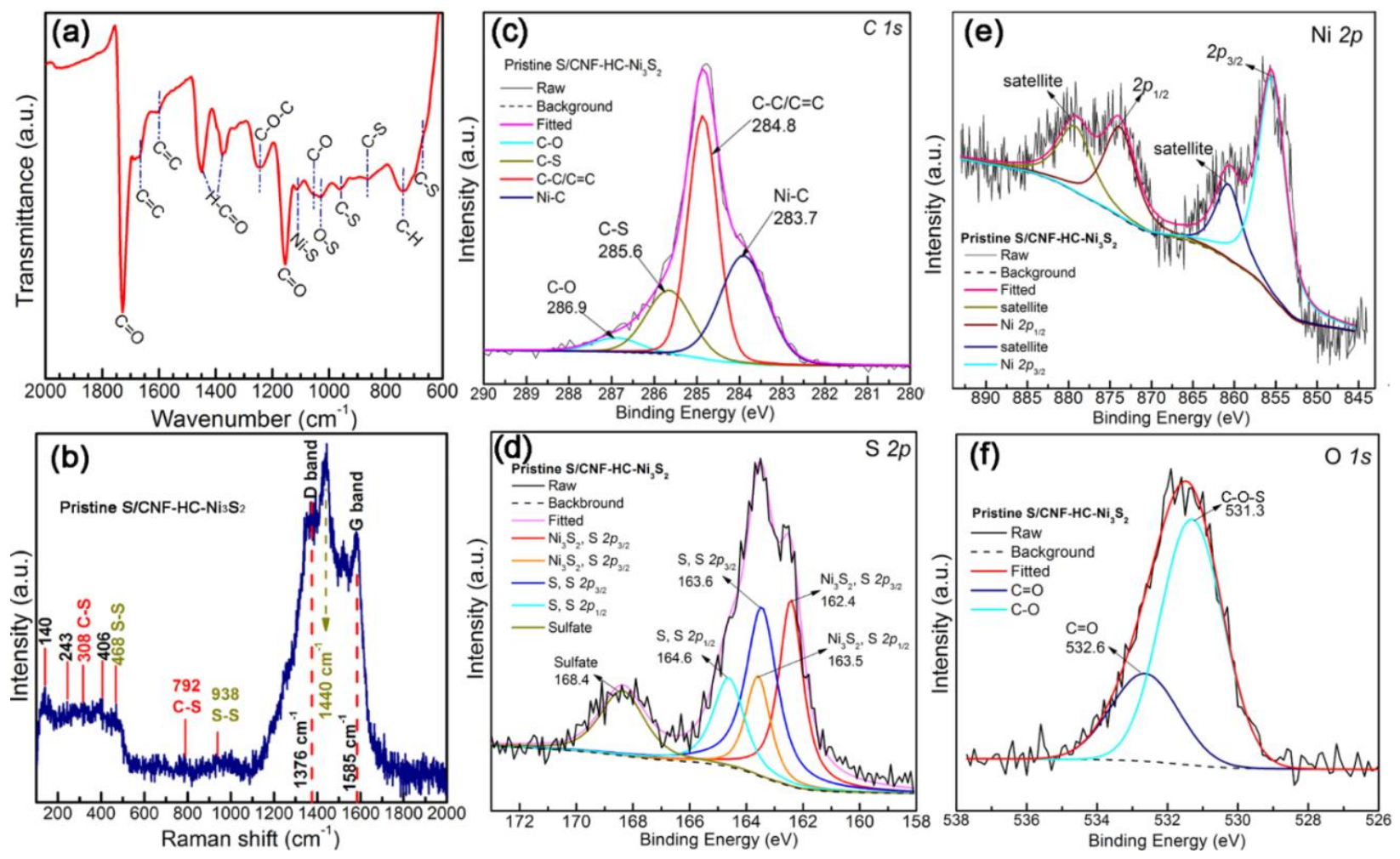

Figure 40 FTIR (a) and Raman spectra (b), and high-resolution XPS spectra of C $1 s$ (c), S $2 p$ (d),

$\mathrm{Ni} 2 p(\mathrm{e})$ and $\mathrm{O} 1 s(\mathrm{f})$ peaks of pristine $\mathrm{S} / \mathrm{CNF}-\mathrm{HC}-\mathrm{Ni}_{3} \mathrm{~S}_{2}$ electrode.

\subsection{Electrochemical performance with low and high sulfur loading}

The electrochemical performances of $\mathrm{S} / \mathrm{CNF}-\mathrm{HC}-\mathrm{Ni}_{3} \mathrm{~S}_{2}$ were systemically investigated as cathodes of Li-S batteries. To study the role of $\mathrm{Ni}_{3} \mathrm{~S}_{2}, \mathrm{~S} / \mathrm{CNF}-\mathrm{HC}$ was also prepared as a control sample with similar morphology and architecture except for the absence of $\mathrm{Ni}_{3} \mathrm{~S}_{2}$. Figure 41a shows the first five cyclic voltammograms (CV) curves of the S/CNF-HC- $\mathrm{Ni}_{3} \mathrm{~S}_{2}$ electrode at a scan rate of $0.1 \mathrm{mV} \mathrm{s}^{-1}$ between 1.7 and 3.0 V. Two sharp cathodic peaks at $\sim 2.3$ (I) and $\sim 2.0$ (II) $\mathrm{V}$ can be observed, corresponding to the reduction of $\mathrm{S}_{8}$ to long-chain $\operatorname{LiPSs}\left(\operatorname{Li}_{2} \mathrm{~S}_{\mathrm{x}}, 4 \leq \mathrm{x} \leq 8\right)$ and then to insoluble short-chain discharged products $\mathrm{Li}_{2} \mathrm{~S}_{2} / \mathrm{Li}_{2} \mathrm{~S}$. Two anodic peaks appear at $\sim 2.3$ and $\sim 2.4 \mathrm{~V}$, accounting for the oxidation of lithium sulfides to LiPSs and sulfur. ${ }^{[188]}$ After the initial activation cycle, the following successive CV curves are well overlapped, indicating 
the highly reversible redox conversion reactions and constant suppression on electrochemical polarization. ${ }^{[29 b, 189]}$ In contrast, the S/CNF-HC electrode exhibits much broader CV peaks for both cathodic and anodic reactions, apparent peak shifts, and degradation during continuous cycles (Figure 41b), indicative of slow redox kinetics and severe polarization due to the absence of $\mathrm{Ni}_{3} \mathrm{~S}_{2}$ component. It is obvious that the cathodic and anodic peaks of S/CNF-HC-Ni $\mathrm{S}_{3}$ electrode are sharper and narrower, which also confirms the critical role of $\mathrm{Ni}_{3} \mathrm{~S}_{2}$ during the redox reactions. The $\mathrm{CV}$ results signify that $\mathrm{Ni}_{3} \mathrm{~S}_{2}$ can substantially accelerate kinetics, promote redox reversibility and stability, and mitigate polarization in LiPSs redox reactions.

The cycling performances of S/CNF-HC-Ni $\mathrm{S}_{2}$ and S/CNF-HC cathodes at $0.2 \mathrm{C}$ are compared in Figure 41c. The specific capacity of S/CNF-HC cathode is rapidly decreased to only $\sim 400 \mathrm{mAh} \mathrm{g}^{-1}$ after 100 cycles, whereas the S/CNF-HC-Ni $\mathrm{S}_{2}$ electrode still maintains a high specific capacity $\left(c a .850 \mathrm{mAh} \mathrm{g}^{-1}\right.$ ). This result manifests that the design of S/CNF-HC-Ni $\mathrm{S}_{2}$ can effectively mitigate the diffusion of soluble LiPSs and loss of active sulfur, minimize the volume change and structural collapse, thus resulting in the high sulfur utilization, specific capacity and cyclability. The corresponding discharge and charge curves (Figure 41d and e) show characteristic plateaus in good agreement with their respective CV curves as well as the results in the literature. ${ }^{[190]}$ In sharp contrast to S/CNF-HC with exacerbated polarization, the S/CNF-HC-Ni $\mathrm{S}_{2}$ cathode exhibits overlapped discharge/charge voltage stages and low polarization during cycles. This suggests the effective binding and anchoring of LiPSs with abundant active sites, fast redox kinetics, and remarkable reversibility in S/CNF-HC-Ni $\mathrm{S}_{2}$. The rate capability of S/CNF-HC-Ni $\mathrm{S}_{2}$ cathode was evaluated at various rates (Figure 41f) with corresponding charge/discharge curves presented in Figure 41g. S/CNF-HC-Ni $\mathrm{S}_{2}$ exhibits stable and high reversible capacities of 1017.8, 883.8, 787.4, and $688.8 \mathrm{mAh} \mathrm{g}^{-1}$ at $0.2,0.5,1$, and $3 \mathrm{C}$, 
respectively. Even when the current rate increases to $5 \mathrm{C}$, a capacity of $530 \mathrm{mAh} \mathrm{g}^{-1}$ can still be maintained. In addition, the characteristic stable and phased plateaus can still be clearly observed even at $5 \mathrm{C}$, reflecting the enhanced redox kinetics of S/CNF-HC-Ni $\mathrm{S}_{2}$. For the long-term cycle stability at the current density of $5 \mathrm{C}$, the capacities can be maintained at $\sim 400 \mathrm{mAh} \mathrm{g}^{-1}$ after 450 cycles (Figure 41h) with the Coulombic efficiencies close to $100 \%$. Such outstanding rate performance is attributed to the integrated conductive electrode architecture composed of $\mathrm{Ni}$ foam, HC, and CNF layers for providing 3D electron pathway network and to rich active and adsorption sites for facilitating ion transfer and redox kinetics. 

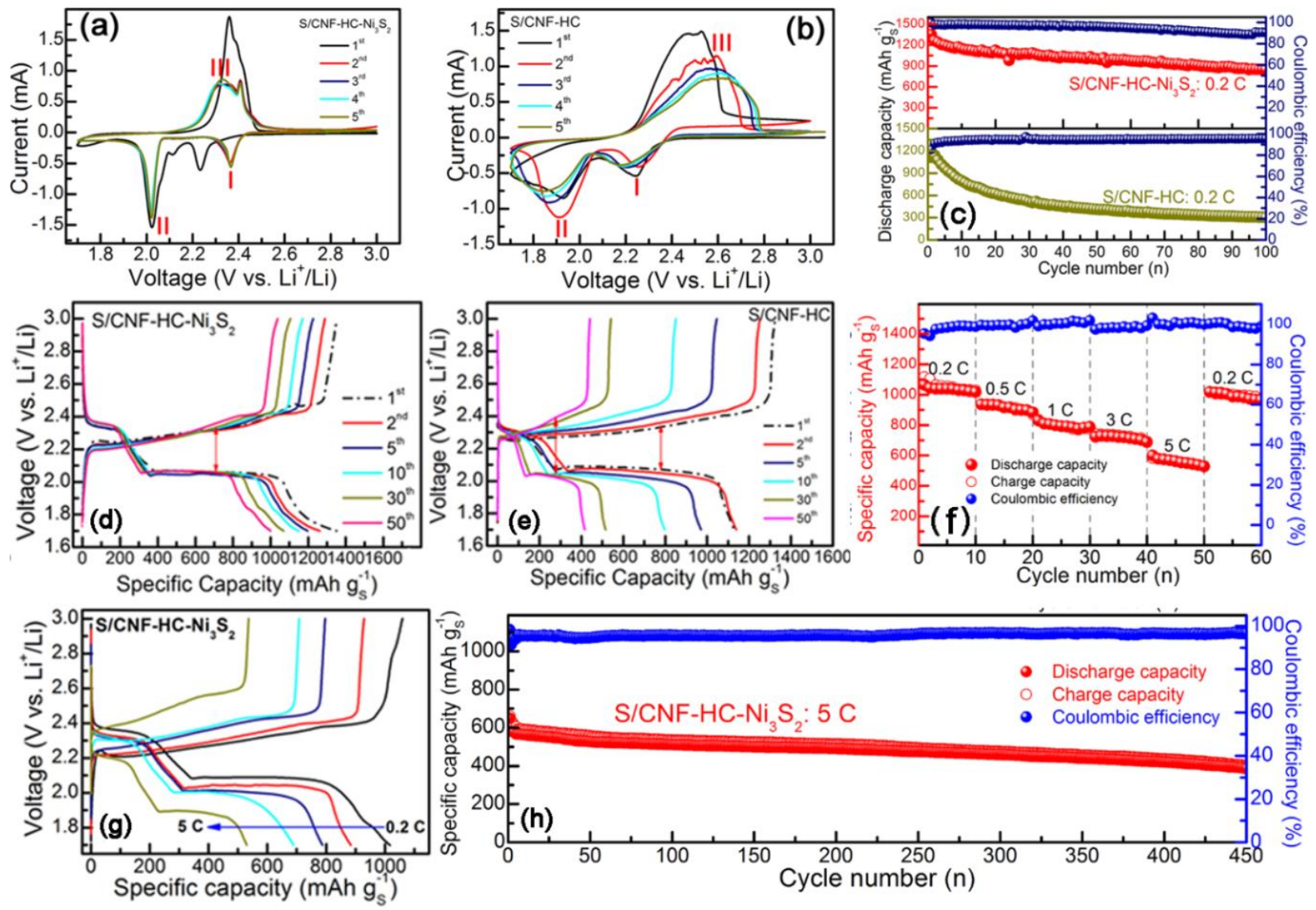

Figure $41 \mathrm{CV}$ curves of S/CNF-HC-Ni $\mathrm{S}_{2}$ (a) and S/CNF-HC (b) cathodes for 5 cycles, the cycle performance of S/CNF-HC-Ni ${ }_{3} \mathrm{~S}_{2}$ and S/CNF-HC cathodes at $0.2 \mathrm{C}$ (c). The charge/discharge curves of S/CNF-HC-Ni ${ }_{3} \mathrm{~S}_{2}$ and S/CNF-HC electrode (d, e). The rate capability (f) and discharge-charge profiles of the S/CNF-HC-Ni $\mathrm{S}_{2}$ electrode at various current densities $(\mathrm{g})$. Long-term cycles of S/CNF-HC-Ni $\mathrm{S}_{2}$ cathode at a current density of $5 \mathrm{C}(\mathrm{h})$ for $\mathrm{Li}-\mathrm{S}$ batteries.

The excellent structure stability of the S/CNF-HC- $\mathrm{Ni}_{3} \mathrm{~S}_{2}$ electrode can be also revealed in Figure 42. The stable high capacity could still be maintained for $\mathrm{S} / \mathrm{CNF}-\mathrm{HC}-\mathrm{Ni}_{3} \mathrm{~S}_{2}$ electrode after the current density switched back from $5 \mathrm{C}$ to $0.2 \mathrm{C}$. A capacity of $810 \mathrm{mAh} \mathrm{g}^{-1}$ can be delivered on return to $0.2 \mathrm{C}$ with $80 \%$ specific capacity retention after 110 cycles (Figure 42a), indicating the excellent robustness and stability of the integrated electrode. Moreover, long-term high rate cycling stability for S/CNF-HC-Ni $\mathrm{S}_{2}$ electrodes was determined at $2 \mathrm{C}$ and $5 \mathrm{C}$. The 
capacities can be maintained at $\sim 620 \mathrm{mAh} \mathrm{g}^{-1}$ (2 C) after 300 cycles (Figure 42b). This accentuates the enhanced cycling stability and redox kinetics primarily owing to the structural design advantages. More remarkably, the host carbon layer acting as the main reservoir for sulfur in $\mathrm{S} / \mathrm{CNF}-\mathrm{HC}-\mathrm{Ni}_{3} \mathrm{~S}_{2}$ can accommodate a relatively high sulfur loading of $\sim 4 \mathrm{mg} \mathrm{cm}^{-2}$, which can sustain a reversible discharge capacity of $\sim 770 \mathrm{mAh} \mathrm{g}^{-1}$ (i.e., $3.2 \mathrm{mAh} \mathrm{cm}^{-2}$ ) at $0.2 \mathrm{C}$ after 100 cycles (Figure 42c and d), presenting the superior design of S/CNF-HC-Ni $\mathrm{S}_{2}$ cathodes with great promise in robust, long-term, and high current load energy applications. Above all, the electrochemical performances of S/CNF-HC-Ni ${ }_{3} \mathrm{~S}_{2}$ stand out among recently reported cathodes for Li-S batteries (Table 6).
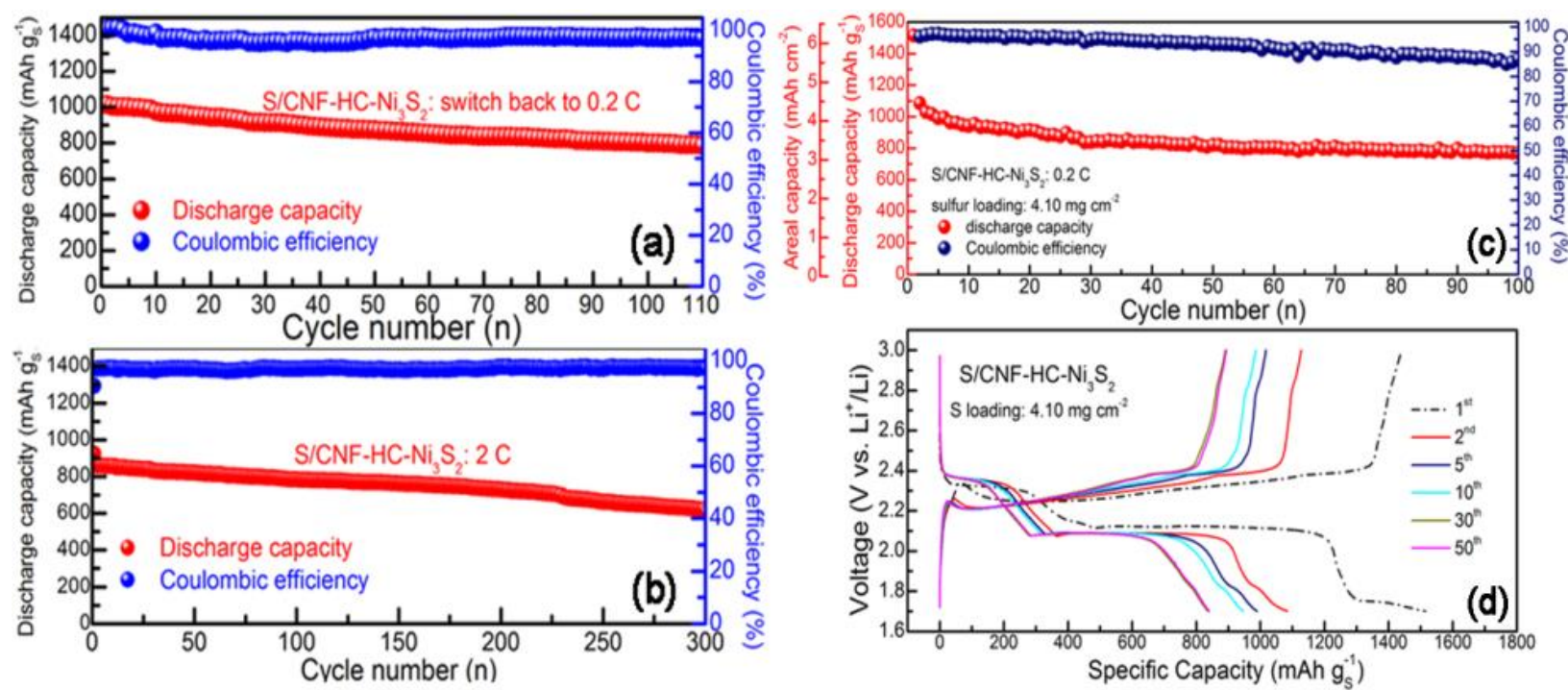

Figure 42 Cycle performance of S/CNF-HC-Ni ${ }_{3} \mathrm{~S}_{2}$ electrodes on return to $0.2 \mathrm{C}$ for 100 cycles (a), and $2 \mathrm{C}$ for 300 cycles (b). Cycle performance of $\mathrm{S} / \mathrm{CNF}-\mathrm{HC}-\mathrm{Ni}_{3} \mathrm{~S}_{2}$ electrode with a relatively high loading tested at $0.2 \mathrm{C}$ (a) and corresponding charge-discharge curves (b). 
Table 6 The performance comparison of this work with some other similar composites.

\begin{tabular}{|c|c|c|c|c|c|}
\hline $\begin{array}{l}\text { Cathodic } \\
\text { materials }\end{array}$ & $\begin{array}{l}\text { Mass loading } \\
\left(\mathrm{mg} \mathrm{cm}^{-2}\right)\end{array}$ & Rate & 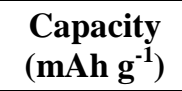 & 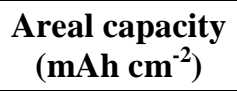 & Reference \\
\hline $\begin{array}{c}\text { S/CNF-HC- } \\
\mathrm{Ni}_{3} \mathrm{~S}_{2}\end{array}$ & $\begin{array}{l}2.31 \\
1.67 \\
4.11 \\
\end{array}$ & $\begin{array}{l}0.2 \mathrm{~A} \mathrm{~g}^{-1} \\
5 \mathrm{~A} \mathrm{~g}^{-1} \\
0.2 \mathrm{~A} \mathrm{~g}^{-1}\end{array}$ & $\begin{array}{l}850(100) \\
433(200) \\
760(100) \\
\end{array}$ & $\begin{array}{l}1.97 \\
0.72 \\
3.12 \\
\end{array}$ & This work \\
\hline S/NiS@C-HS & 1.0 & $0.2 \mathrm{C}$ & $718(200)$ & 0.72 & [119] \\
\hline TiN-S & 4.6 & $0.77 \mathrm{~mA} \mathrm{~cm}^{-2}$ & $685(50)$ & 3.15 & [191] \\
\hline S/IKB & 4.7 & $1.57 \mathrm{~mA} \mathrm{~cm}$ & $800(90)$ & 3.76 & [192] \\
\hline $\mathbf{S}_{2-4} / \mathrm{UMC}$ & 4.2 & $2 \mathrm{~mA} \mathrm{~cm}-2$ & $580(60)$ & 2.44 & [193] \\
\hline $\mathrm{S} / \mathrm{Co}_{9} \mathrm{O}_{8}$ & 4.5 & $0.05 \mathrm{C}$ & $500(150)$ & 2.25 & [194] \\
\hline S/G-DBD & 1.6 & $0.2 \mathrm{C}$ & $754(200)$ & 1.21 & [156] \\
\hline NSF-S & 4.0 & $1 \mathrm{~mA} \mathrm{~cm}^{-2}$ & $654(80)$ & 2.62 & [157] \\
\hline $\begin{array}{l}\text { S@NiCo- } \\
\text { DH@RC }\end{array}$ & 1.5 & $\begin{array}{l}0.2 \mathrm{C} \\
5 \mathrm{C}\end{array}$ & $\begin{array}{l}972(250) \\
698 \text { (rate } \\
\text { capacity) } \\
\end{array}$ & 1.46 & [159] \\
\hline $\mathrm{MgB}_{2}-\mathrm{S60}$ & 2.0 & $0.5 \mathrm{C}$ & $\sim 800(200)$ & 1.6 & {$[160]$} \\
\hline S/PPA & 1.5 & $0.5 \mathrm{C}$ & $763(100)$ & 1.14 & {$[161]$} \\
\hline $\mathrm{Li}_{2} \mathrm{~S}-\mathrm{CoSe}_{2} / \mathrm{G}$ & 4.35 & $0.5 \mathrm{C}$ & $832(100)$ & 3.62 & {$[163]$} \\
\hline TiN-C65 & 0.6 & $0.2 \mathrm{C}$ & $680(100)$ & 0.41 & [168] \\
\hline PRC/Ni/S & 4.0 & $0.2 \mathrm{C}$ & $564(300)$ & 2.26 & [195] \\
\hline S/CNT/PrNP & $\begin{array}{l}2.5 \\
3.8 \\
5.2\end{array}$ & $\begin{array}{l}0.2 \mathrm{C} \\
0.2 \mathrm{C} \\
0.2 \mathrm{C}\end{array}$ & $\begin{array}{l}800(200) \\
685(200) \\
711(100)\end{array}$ & $\begin{array}{l}2.0 \\
2.6 \\
3.7\end{array}$ & [166] \\
\hline $\mathrm{S} @ \mathbf{N i} / \mathbf{F e} \mathbf{L D H}$ & 2.3 & $0.2 \mathrm{C}$ & $725(200)$ & 1.64 & {$[172]$} \\
\hline CNF sheets/S & 2.4 & $0.33 \mathrm{C}$ & $683(500)$ & 1.64 & [190b] \\
\hline S/C with NCM & 4.0 & $0.5 \mathrm{C}$ & $\sim 550(100)$ & 2.2 & {$[170]$} \\
\hline $\begin{array}{l}\text { S/CNT with } \\
\text { PNG }\end{array}$ & 3.6 & $1.5 \mathrm{~mA} \mathrm{~cm}^{-2}$ & $688(400)$ & 2.48 & [196] \\
\hline C@TiN-S & 4.2 & $0.2 \mathrm{C}$ & $820(150)$ & 3.44 & [189a] \\
\hline $\mathrm{MoS}_{2} / \mathrm{rGO} / \mathrm{S}$ & 0.85 & $0.5 \mathrm{C}$ & $680(200)$ & 0.58 & [190a] \\
\hline
\end{tabular}




\subsection{Analysis of redox kinetics and polysulfide trapping ability}

In-depth electrochemical experiments and post-mortem analyses were performed to anatomize the reasons for the outstanding electrochemical performances of S/CNF-HC-Ni $\mathrm{S}_{2}$ cathodes, which will shed light on the mechanistic insights for guiding rational and competent cathode designs in the future. First, the well-distributed $\mathrm{Ni}_{3} \mathrm{~S}_{2}$ particles can serve as a highly efficient electrocatalyst with high electrocatalytic activity for both reducing the energy barriers and facilitating the kinetics for LiPSs-involved redox reactions. To elucidate the electrocatalytic effects, the peak voltages of S/CNF-HC-Ni $\mathrm{S}_{2}$ and S/CNF-HC electrodes for two cathodic peaks (I and II) and one anodic peak (III) derived from their CV curves (Figure 41) are compared (Figure 43a). The presence of $\mathrm{Ni}_{3} \mathrm{~S}_{2}$ can raise the discharge voltages of cathodic peaks by at least $170 \mathrm{mV}$ and reduce the charge voltage of anodic peak by $270 \mathrm{mV}$. In this case, $\mathrm{Ni}_{3} \mathrm{~S}_{2}$ can substantially mitigate the polarization from 0.73 to $0.29 \mathrm{~V}$ (i.e., voltage hysteresis between III and II). These results are consistent with those of the galvanostatic discharge-charge profiles (Figure 41), suggesting that $\mathrm{Ni}_{3} \mathrm{~S}_{2}$ can weaken the energy barriers for redox reactions. The onset potential was taken at a current density of $10 \mu \mathrm{A} \mathrm{cm} \mathrm{cm}^{-2}$ beyond the baseline current, determined by a reported method. ${ }^{[112]}$ Likewise, $\mathrm{Ni}_{3} \mathrm{~S}_{2}$ contributes to the increased onset potentials of cathodic peaks (I and II) and decreased onset potential of anodic peak (III) (Figure 43b). Such trends are more evident in the comparison of their polarization curves (Figure $43 \mathrm{c}$ and d). Tafel plots were obtained from the polarization curves to uncover the electrocatalytic effect of $\mathrm{Ni}_{3} \mathrm{~S}_{2}$ on the charge transfer kinetics in LiPSs-involved redox reactions. S/CNF-HC-Ni $\mathrm{S}_{2}$ shows Tafel slopes of 75 and $62 \mathrm{mV} \mathrm{dec}^{-1}$ in the reduction (I) and oxidation (III) processes (Figure 43e), respectively, much smaller than those of S/CNF-HC counterpart, implying the promoted kinetics over the $\mathrm{Ni}_{3} \mathrm{~S}_{2}$ electrocatalyst. Moreover, the redox kinetics and charge transfer were examined 
by $\mathrm{CV}$ (scan rate: $10 \mathrm{mV} \mathrm{s}^{-1}$ ) in symmetric cells, using two identical electrodes with $\mathrm{Li}_{2} \mathrm{~S}_{6}$ electrolyte. To eliminate the influence from the capacitive background current in the CV curves, a symmetric cell with the $\mathrm{Li}_{2} \mathrm{~S}_{6}$-free electrolyte and CNF-HC-Ni $\mathrm{S}_{2}$ electrode couple was also measured, presenting negligible current density (Figure 43f). The current density of CNF-HC$\mathrm{Ni}_{3} \mathrm{~S}_{2}$ symmetric cells is much higher than that of the CNF-HC control sample, indicative of notable enhancement on the redox reactions of LiPSs. The Nyquist plots in the EIS of symmetrical cells further confirm the boosted charge transfer process at the $\mathrm{Li}_{2} \mathrm{~S}_{6} / \mathrm{CNF}-\mathrm{HC}$ $\mathrm{Ni}_{3} \mathrm{~S}_{2}$ interface, with a significantly lower charge transfer resistance $\left(\mathrm{R}_{\mathrm{ct}}\right)$ of $168 \Omega \mathrm{cm}^{2}$ compared to that of CNF-HC $\left(2214 \Omega \mathrm{cm}^{2}\right)$. It is clear that the intimate coupling of welldistributed sulfiphilic $\mathrm{Ni}_{3} \mathrm{~S}_{2}$ and electrically conductive $\mathrm{HC}$ and CNF matrices can readily promote the access of polysulfide ions and electron transfer to $\mathrm{LiPSs} / \mathrm{Ni}_{3} \mathrm{~S}_{2}$ interface to trigger the LiPSs redox reactions, unambiguously demonstrating expedited redox conversion kinetics and charge transfer. Collectively, these electrochemical analyses verify that $\mathrm{Ni}_{3} \mathrm{~S}_{2}$ plays pivotal roles in electrocatalytically decreasing the energy barriers and accelerate kinetics for LiPSsinvolved redox reactions. Second, the S/CNF-HC-Ni $\mathrm{S}_{2}$ cathode possesses rich anchoring and adsorption sites with strong chemical LiPSs binding capability for immobilizing soluble LiPSs at the interfacial sites through interactions primarily with polar $\mathrm{Ni}_{3} \mathrm{~S}_{2}$ and possible C-S bonds. To unravel the interactions, the XPS characterization of S/CNF-HC-Ni ${ }_{3} \mathrm{~S}_{2}$ cathode after 100 cycles at the discharged state was conducted. For C $1 s$ spectrum (Figure $43 \mathrm{~h}$ ), besides the $\mathrm{C}-\mathrm{C} / \mathrm{C}=\mathrm{C}$ bonds from the carbon host, a strong peak corresponding to C-S bonding can be identified at $286.0 \mathrm{eV}$, which is shifted towards higher binding energy by $0.4 \mathrm{eV}$ compared to that of the pristine electrode, together with the emerging carbonate species on the cycled electrode. This signifies the interaction of polysulfide with sulfurized carbon hosts. ${ }^{[175]}$ The $\mathrm{S} 2 p$ spectrum 
(Figure 43i) shows three major peaks of discharged lithium polysulfide and sulfide on the surface of the cycled cathode, centered at $162.7,161.3$, and $160.1 \mathrm{eV}$, corresponding to the residual bridging $\left(\mathrm{S}_{\mathrm{B}}^{0}\right)$, terminal $\left(\mathrm{S}_{\mathrm{T}}^{-1}\right) \mathrm{S}$ species and sulfides, respectively. ${ }^{[53,176,197]}$ These binding energies are lower than those of pristine counterparts, indicating the chemical trapping of LiPSs species on the S/CNF-HC-Ni $\mathrm{S}_{2}{ }^{[159,}$ 175b, 176]
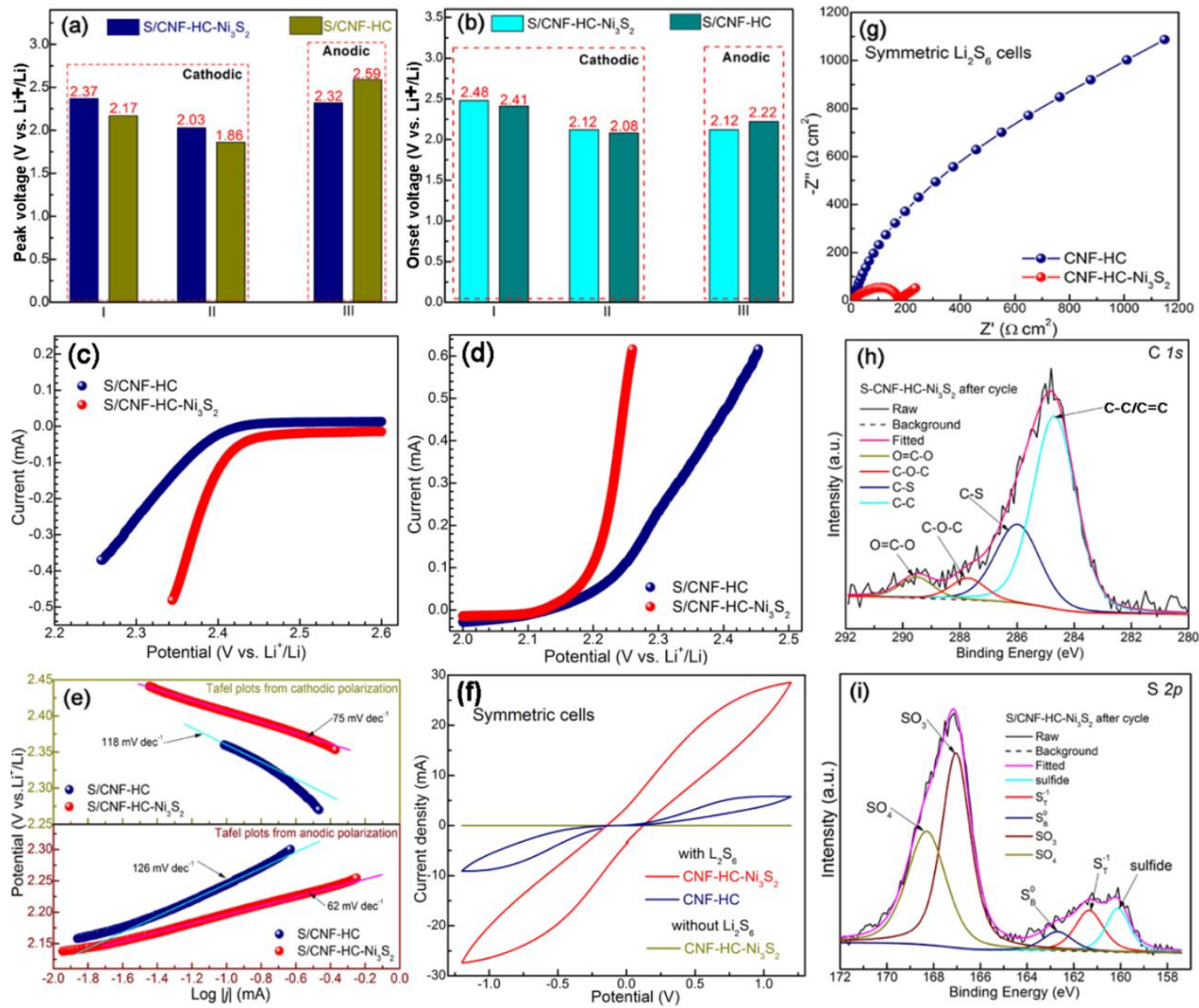

Figure 43 Comparison of CV peak voltages (a), onset potentials (b), cathodic (c) and anodic (d) polarization curves and Tafel plots (e) of asymmetrical Li-S cells consisting of both S/CNF-HC$\mathrm{Ni}_{3} \mathrm{~S}_{2}$ or $\mathrm{S} / \mathrm{CNF}-\mathrm{HC}$ cathode and $\mathrm{Li}$ anode. (f) $\mathrm{CV}$ curves and (g) EIS Nyquist curves of symmetric cells of CNF-HC-Ni $\mathrm{S}_{2}$ or CNF-HC electrodes. (h) C $1 s$ and (i) $\mathrm{S} 2 p$ XPS spectra of S/CNF-HC-Ni $\mathrm{S}_{2}$ cathode in asymmetrical Li-S cells after 100 cycles at discharged state. 
To further visualize the suppressive effects of $\mathrm{Ni}_{3} \mathrm{~S}_{2}$ on the shuttling of LiPSs, Figure 44 compares the photographs of cycled Li-S cells based on S/CNF-HC-Ni $\mathrm{S}_{2}$ and other control samples. The S/CNF-HC cathode (Figure 44a) contains visible white sulfur spots and yellowgreen soluble LiPSs solution can be found when the cycled cathode was soaked in dimethoxyethane (DME). Meanwhile, black contaminants adhere to the separator and the Li foil has been seriously corroded, indicating the severe migration of LiPSs towards Li anode due to the weak binding capability of S/CNF-HC in the absence of $\mathrm{Ni}_{3} \mathrm{~S}_{2}$ chemical anchors. Indeed, S/CNF-HC delivers fast capacity decay during cycles (Figure 44c). Previous reports have also confirmed that the incompatibility in the surface affinity of nonpolar carbon with polar polysulfides makes it incapable of effectively inhibiting LiPSs migration and flooding in pure carbon-based sulfur cathodes. ${ }^{[83,103,198]}$ In stark contrast, the S/CNF-HC-Ni ${ }_{3} \mathrm{~S}_{2}$ electrode (Figure 44b) after 100 cycles shows few visible sulfur species, and its solution is slightly colored when soaked in DME solvent. The separator and $\mathrm{Li}$ anode are much cleaner, demonstrating the extraordinary chemical adsorption and binding capabilities for anchoring polysulfides. The binding energies of multiple LiPSs on the $\mathrm{Ni}_{3} \mathrm{~S}_{2}$ surface at the molecular level have been calculated through the density functional theory (DFT) in previous reports which are much higher than those on the nonpolar carbon surface, ${ }^{[91 b, 157]}$ revealing an inherent energetically favorable interaction between LiPSs and $\mathrm{Ni}_{3} \mathrm{~S}_{2}$. These results confirm the superior adsorption and binding capabilities of S/CNF-HC-Ni ${ }_{3} \mathrm{~S}_{2}$ for LiPSs, which are responsible for the phenomenal cycle stability and high utilization of sulfur. 


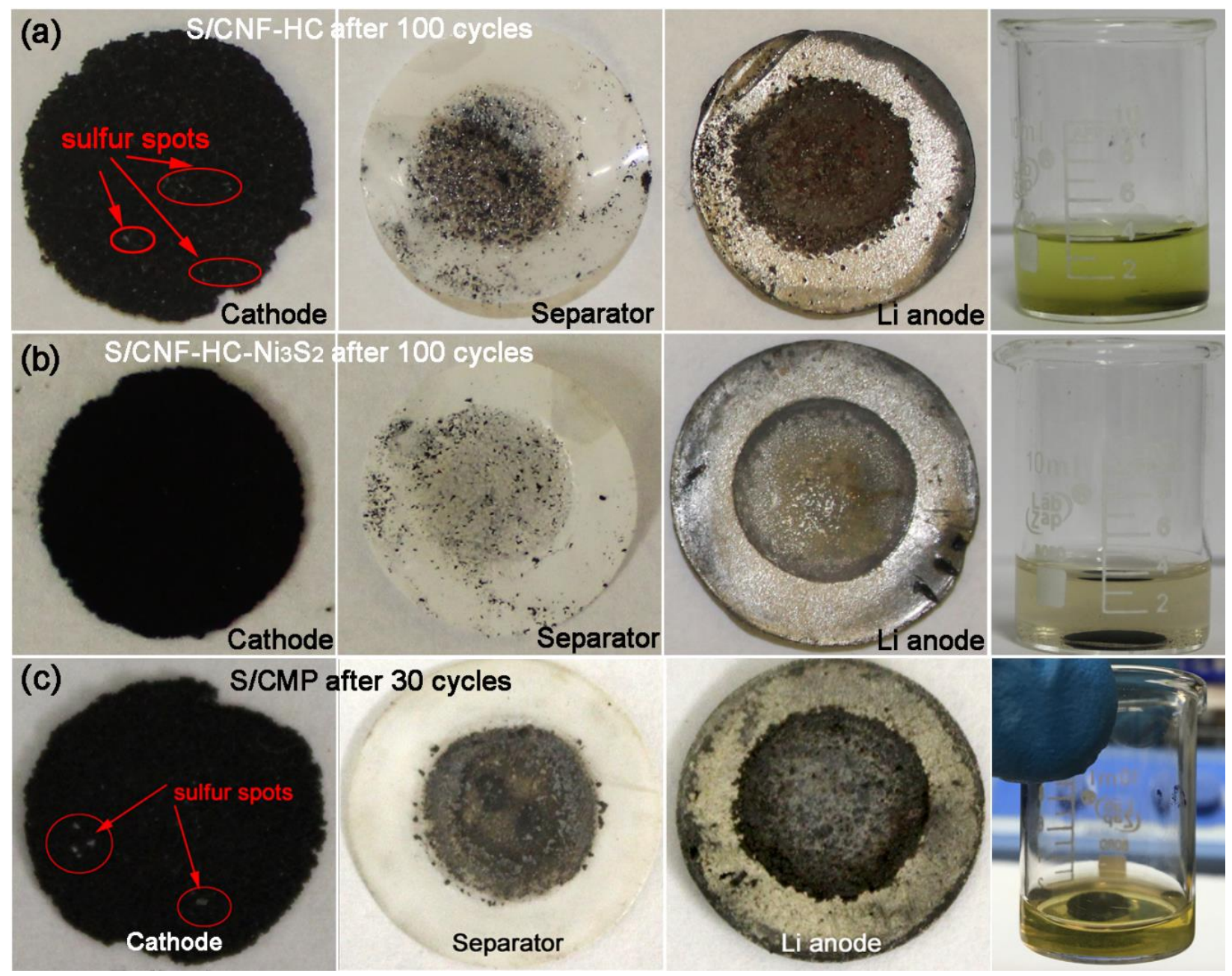

Figure 44 Photographs of the cycled Li-S cells of (a) S/CNF-HC and (b) S/CNF-HC-Ni ${ }_{3} \mathrm{~S}_{2}$ cathodes after 100 cycles and (c) S/CMP after 30 cycles with the corresponding separators, Li anodes and respective visualized cathodes soaked in dimethoxyethane solvent.

Third, the S/CNF-HC-Ni $\mathrm{S}_{2}$ cathode holds cooperative interfaces of "lithiophilic" heteroatomdoped carbon and "sulfiphilic" $\mathrm{Ni}_{3} \mathrm{~S}_{2}$, which can help to address the shuttle and kinetics issues synchronously by binding polysulfides and enhancing affinity to $\mathrm{Li}$ (e.g., $\mathrm{Li}^{+}$ions and/or terminal $\mathrm{Li}$ in LiPSs) and transport of charge carriers. As confirmed by the spectroscopic characterization in Figure 40, the pristine S/CNF-HC-Ni $\mathrm{S}_{2}$ electrode contains heteroatom-doped carbon (e.g., C-S and C-O bonding), which is found to exhibit desirable lithiophilicity via Li-S or 
Li-O bonds. ${ }^{[47 b, 80,97,152 c, 199]}$ Meanwhile, $\mathrm{Ni}_{3} \mathrm{~S}_{2}$ demonstrates favorable sulfiphilicity to bind the terminal $\mathrm{S}$ of LiPSs with exposed Ni sites via Ni-S bonds, which is supported by reported results for hybrid metal sulfide-LiPS and other metal-site-containing host-LiPSs systems. ${ }^{[47 c, 70,112,157]}$ Such binary cooperative complementary with distinct chemisorptivity is conducive to enriching $\mathrm{Li}$ local concentration in the vicinity of cathode surface for promoting $\mathrm{Li}^{+}$-transfer induced kinetics and also to immobilizing LiPSs on the heterogeneous surfaces for facilitating electrocatalytic redox conversion and restraining LiPSs shuttling. The cooperative interfaces of "lithiophilic" heteroatom-doped carbon and "sulfiphilic" $\mathrm{Ni}_{3} \mathrm{~S}_{2}$ for interaction with LiPSs are tentatively suggested by the XPS peak shifts of C $1 s(\mathrm{C}-\mathrm{S}), \mathrm{S} 2 p$ and Ni $2 p_{3 / 2}$ and the changes of $\mathrm{Li} l s$ and $\mathrm{O} l s(\mathrm{O}-\mathrm{Li})$ of $\mathrm{S} / \mathrm{CNF}-\mathrm{HC}-\mathrm{Ni}_{3} \mathrm{~S}_{2}$ cathode after 100 cycles. $^{[152 \mathrm{c}]}$ The cooperative interfaces are proposed to play critical roles in the enhanced cycling stability (Figure 41) and accelerated redox kinetics as well as reduced charge transfer resistance (Figure 43) of S/CNF$\mathrm{HC}-\mathrm{Ni}_{3} \mathrm{~S}_{2}$ beyond those of S/CNF-HC. Fourth, the binder-free freestanding S/CNF-HC-Ni $\mathrm{S}_{2}$ electrode architecture containing electronically conductive Ni foam framework, $\mathrm{HC}$ and CNF layers provides an efficient 3D electron pathway network, enabling fast electron transport to interfacial adsorbed LiPSs and $\mathrm{Ni}_{3} \mathrm{~S}_{2}$ electrocatalyst for fast redox kinetics and remarkable rate capability. Moreover, $\mathrm{Ni}_{3} \mathrm{~S}_{2}$ also has a fairly low resistivity $\left(1.8 \times 10^{-5} \Omega \mathrm{cm}\right.$ at room temperature). ${ }^{[157,200]}$ Such 3D interconnected continuous electron channels are benefited from the strong coupling of various components, rendering electrons to readily reach the LiPSs adsorption interfaces and electrocatalytic active sites and hence promoting the charge transfer and redox kinetics. In combination with the strong LiPSs binding capability and cooperative interfaces, the conductive network S/CNF-HC-Ni $\mathrm{S}_{2}$ electrode ensures its accelerated redox kinetics and small $\mathrm{R}_{\mathrm{ct}}$ (Figure $43 \mathrm{~g}$ ) and exceptional rate performance (Figure 41d). 
Above all, the elaborate structural design of S/CNF-HC-Ni $\mathrm{S}_{2}$ integrates all building blocks of $\mathrm{Ni}$ foam, sulfurized $\mathrm{HC} / \mathrm{CNF}$ forest, $\mathrm{Ni}_{3} \mathrm{~S}_{2}$ particles, and $\mathrm{S}$ with their respective functions into an ensemble, demonstrating a synergistic effect on the outstanding cathode performances in Li-S cells. Ni foam acts as a robust and conductive framework. The heteroatom-doped HC layer with the desired lithiophilicity and electronic conductivity serves as a primary reservoir for loading of active sulfur, helps to bind LiPSs and enables fast electron transport to interfacial adsorbed LiPSs and $\mathrm{Ni}_{3} \mathrm{~S}_{2}$ sites. The sulfurized CNF forest with analogous lithiophilicity and electronic conductivity can increase access to the electrolyte, shorten the electron transport, facilitate the Li-ion transport and retard the LiPSs diffusion as a barrier layer. Sulfiphilic $\mathrm{Ni}_{3} \mathrm{~S}_{2}$ acts as both a chemical anchor with strong chemical LiPSs binding capability for immobilizing soluble LiPSs at the interfacial sites and an efficient electrocatalyst with high catalytic activity for reducing the energy barriers and facilitating the kinetics of redox reactions.

To highlight the structural merits of $\mathrm{S} / \mathrm{CNF}-\mathrm{HC}-\mathrm{Ni}_{3} \mathrm{~S}_{2}$, two control samples, bare $\mathrm{Ni}_{3} \mathrm{~S}_{2} / \mathrm{Ni}$ and Ni foam coated by carbon microspheres layer with sulfur (S/CMP) electrodes together with the aforementioned S/CNF-HC cathode were prepared by modified procedures for comparison. The $\mathrm{Ni}_{3} \mathrm{~S}_{2} / \mathrm{Ni}$ electrode contributes to limited capacity (4-6 $\mathrm{mAh} \mathrm{g}^{-1}$ ) within the potential window of 1.7-3.0 V (Figure 45). The S/CMP electrode without functional units of $\mathrm{HC}, \mathrm{CNF}$, and $\mathrm{Ni}_{3} \mathrm{~S}_{2}$ contains crystalline bulk sulfur in the macropores of $\mathrm{Ni}$ foam and on the surface of CMP, exhibiting rapid decay of capacity and much lower Coulombic efficiency (Figure 46). The polysulfide shuttle resulted in the seriously contaminated separator and corroded Li anode with the dark yellow LiPSs solution when soaked the S/CMP electrode only after 30 cycles in the DME solvent (Figure 44c). In the absence of $\mathrm{Ni}_{3} \mathrm{~S}_{2}$ as the chemical anchor and electrocatalyst, S/CNF-HC cathode also shows poor cycling stability (Figure 41c) and inferior redox kinetics 
(Figure 43). These results indicate the severe shuttling of LiPSs with low sulfur utilization and sluggish redox kinetics in the cathodes without functional building blocks. In contrast, S/CNFHC-Ni $\mathrm{S}_{2}$ exhibits not only remarkable cycle stability and rate capability but also good structure stability. After 100 cycles, S/CNF-HC-Ni ${ }_{3} \mathrm{~S}_{2}$ still maintains the texture (Figure 44b) and surface structure with intact $\mathrm{CNFs}$ and $\mathrm{Ni}_{3} \mathrm{~S}_{2}$ which securely bind the lithium polysulfide and/or sulfide (Figure 47) without any bulk sulfur species aggregated on the surface (Figure 48), indicating little pulverization and volume changes in the electrode. Synergistically, all building blocks of S/CNF-HC-Ni ${ }_{3} \mathrm{~S}_{2}$ promote the lithium-ion coupled electron transfer for redox conversion and retention of LiPSs intermediates in the Li-S battery electrochemistry.

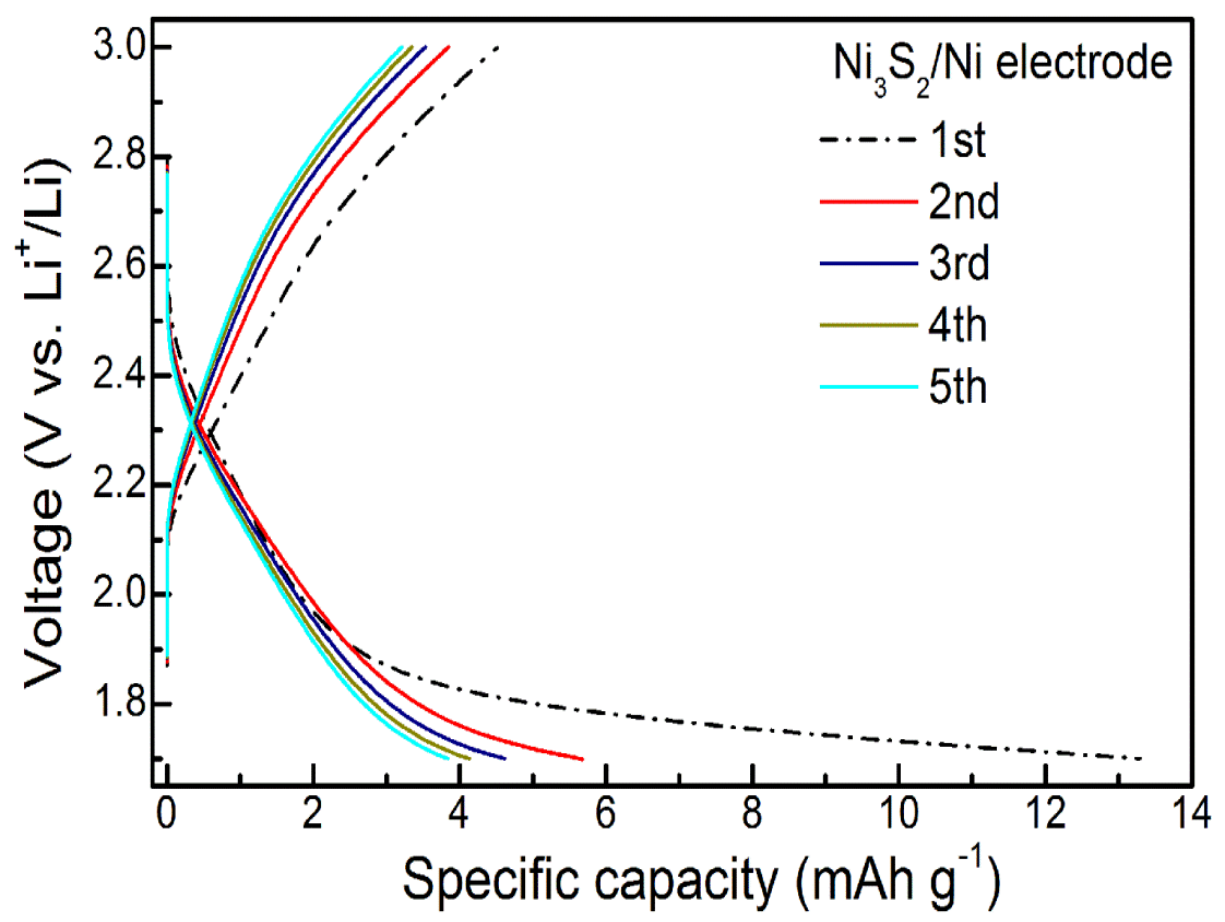

Figure 45 Charge/discharge profiles for the $\mathrm{Ni}_{3} \mathrm{~S}_{2} / \mathrm{Ni}$ electrode. The $\mathrm{Ni}_{3} \mathrm{~S}_{2} / \mathrm{Ni}$ electrodes were prepared by directly mixing the Ni foam discs and sulfur powder for the thermal sulfurization at $300{ }^{\circ} \mathrm{C}$ for $1 \mathrm{~h}$ in $\mathrm{Ar}$. The $\mathrm{Ni}_{3} \mathrm{~S}_{2} / \mathrm{Ni}$ electrode contributes to limited capacity within the potential window of 1.7-3.0 V for testing Li-S cells. 

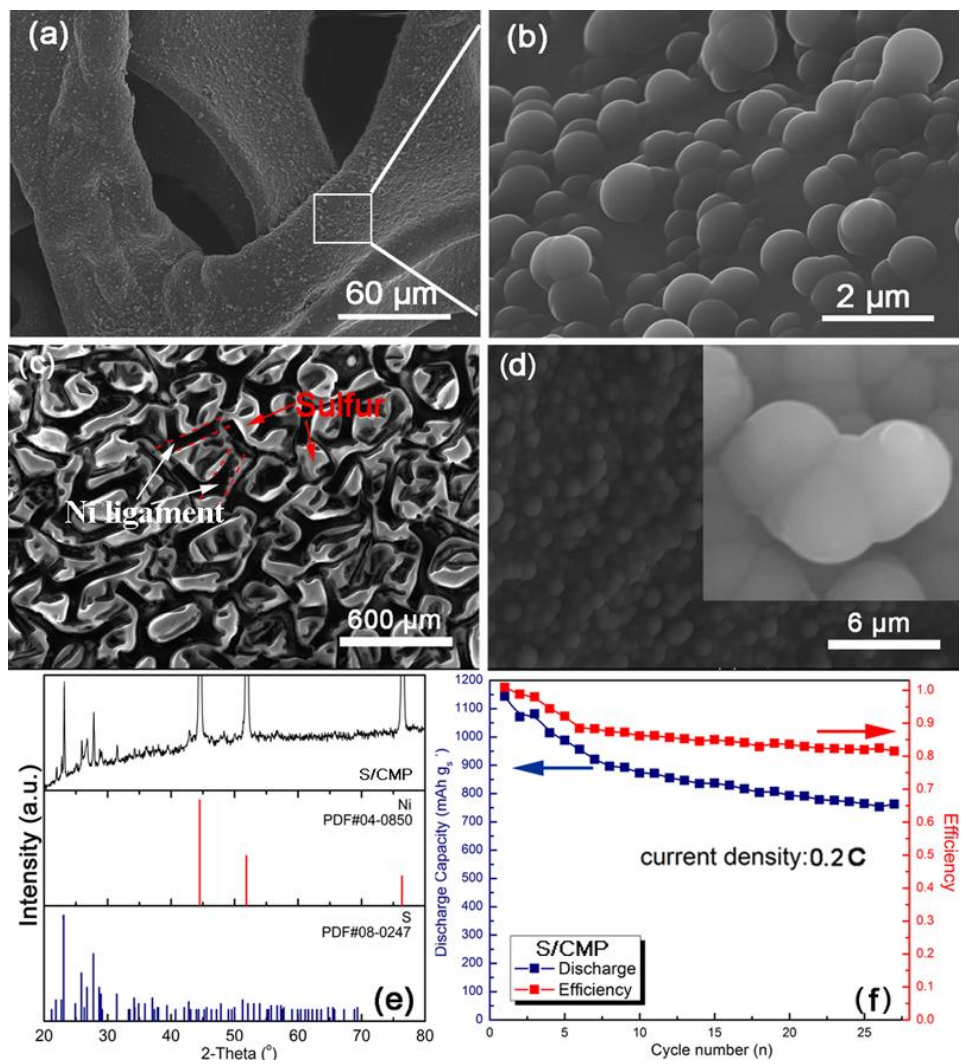

Figure 46 SEM images of CMPs coated Ni foam at low (a) and high (b) magnification. SEM images of S/CMP electrode at low (c) and high (d) magnification. (e) XRD pattern of pristine S/CMP electrode and the corresponding cycle performance at $0.2 \mathrm{C}$ (f).

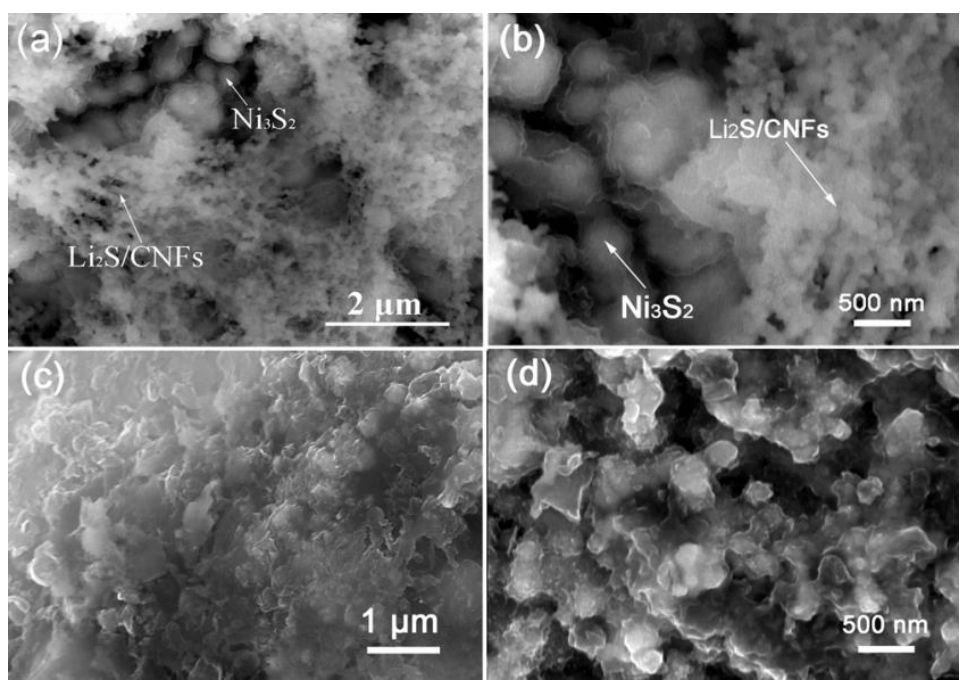

Figure $47 \mathrm{SEM}$ images of S/CNF-HC-Ni $\mathrm{S}_{2}(\mathrm{a}-\mathrm{b})$ and S/CMP (c-d) electrodes after 100 cycles. 


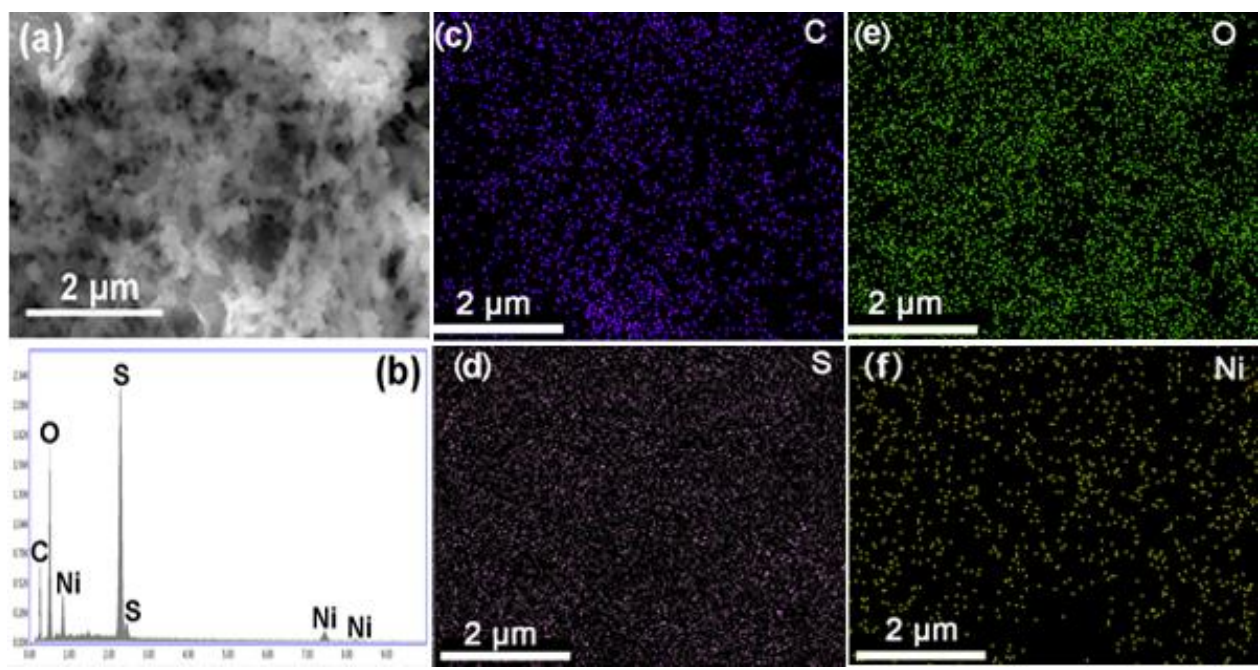

Figure 48 SEM image (a), EDX spectrum (b) of S/CNF-HC-Ni $\mathrm{S}_{2}$ electrode and corresponding elemental maps with C (c), S (d), O (e), and Ni (f) after 100 cycles.

\subsection{Conclusions}

In summary, a bioinspired design of electrode with a hierarchical structure is developed to integrate multiple functional units of $\mathrm{Ni}$ foam, $\mathrm{HC}, \mathrm{CNF}$ forest, $\mathrm{Ni}_{3} \mathrm{~S}_{2}$, and sulfur into an ensemble to obtain a versatile and high-performance cathode $\left(\mathrm{S} / \mathrm{CNF}-\mathrm{HC}-\mathrm{Ni}_{3} \mathrm{~S}_{2}\right)$ for $\mathrm{Li}-\mathrm{S}$ batteries through a programmed fabrication approach. These building blocks have respective specialized functions. Overall, such integrated electrode demonstrates a synergy and thus provides a robust and effective 3D conductive network and cooperative interfaces to minimize the shuttle effect and enhance the rate and cycling performances by increasing the density of adsorption sites, adsorption capability, electron/ion transfer and catalytic redox kinetics for the sulfur species during the discharge-charge process. Due to such unique structural design, the $\mathrm{S} / \mathrm{CNF}-\mathrm{HC}-\mathrm{Ni}_{3} \mathrm{~S}_{2}$ cathode delivers high reversible capacities of $\sim 850 \mathrm{mAh} \mathrm{g}^{-1}$ at $0.2 \mathrm{C}$ after 100 cycles and $\sim 400 \mathrm{mAh} \mathrm{g}^{-1}$ at $5 \mathrm{C}$ after 450 cycles. This work provides a promising cathode candidate and a novel programmed fabrication strategy for the rational design of versatile electrodes for high-energy Li-S batteries. 


\section{Chapter 6: Li metal anode modification}

\subsection{Stable Li metal anode enabled by ionic conducting high entropy oxide protective layer}

\subsubsection{Introduction}

To address the challenges towards the practical application of $\mathrm{Li}$ metal anodes, i.e., the $\mathrm{Li}$ dendrites growth, low Coulombic efficiency, and large volume change during cycling, one of the most important methods is to stabilize the interface between Li anode and electrolyte. The in situ formed SEI films are brittle and crack easily due to the repeated volume change during the $\mathrm{Li}$ stripping and plating process. As illustrated in Figure 49a, a thin SEI film can be quickly formed on the surface of Li foil. However, upon cycling, the SEI film breaks with newly formed lithium dendrites. These dendrites irregularly distribute on the surface and cause a significant difference of $\mathrm{Li}^{+}$flux in the local environment. With the following stripping process, the dendrites dissolved into the electrolyte to form "dead $\mathrm{Li}$ " and a much more unstable interface structure. A promising strategy is to introduce an ex-situ coated protective layers ("artificial SEI") with superior chemical stability, high $\mathrm{Li}^{+}$conductivity, mechanical strength, and flexibility on the $\mathrm{Li}$ metal. An ideal protective layer for the Li metal anode is electronically insulating but ionconducting. A membrane coated on Li that only conducts Li-ions can limit the Li deposition to the region beneath the protective layer, continuously suppressing the growth of Li dendrites. In recent years, the concept of entropy stabilization of crystal structures in oxide systems has led to increased research activity in the field of "high entropy oxides" (HEO). These compounds comprise the incorporation of multiple metal cations into single-phase crystal structures and interactions among the various metal cations leading to interesting novel and unexpected properties. Additionally, the electrochemical behavior of the HEO can be easily tailored by just simply changing the metal cations composition. 
Herein, we report a polymer/high entropy oxide membrane with high $\mathrm{Li}^{+}$conductivity as a protective layer for the Li metal anode and demonstrate that it can remarkably enhance the stability of the anode. As shown in Figure 49b, the protective layer consists of the HEO particles $\left[(\mathrm{MgCoNiZnCu})_{1-\mathrm{x}} \mathrm{Li}_{\mathrm{x}} \mathrm{O}\right]$ and poly(ethylene oxide) (PEO)-based polymer electrolyte. This composite film can not only accommodate large Li volume changes due to the flexible nature of the PEO but also enhance the mechanical properties due to the ceramic oxides. The glassy particles dispersed in the PEO electrolyte with high $\mathrm{Li}^{+}$conductivity but electronically insulating can lead a uniform Li deposition beneath this protective layer, thus suppressing the growth of the Li dendrites. Another superior property of HEO is the highly disordered local crystallization, which is much more stable in the system and favorable for $\mathrm{Li}^{+}$transfer with a uniform $\mathrm{Li}^{+}$flux while inhibiting the electron flow. Therefore, the Li dendrite formation is significantly reduced due to the stable interface structure, highly flexible and mechanical properties, and regulated uniform $\mathrm{Li}^{+}$flux flow.
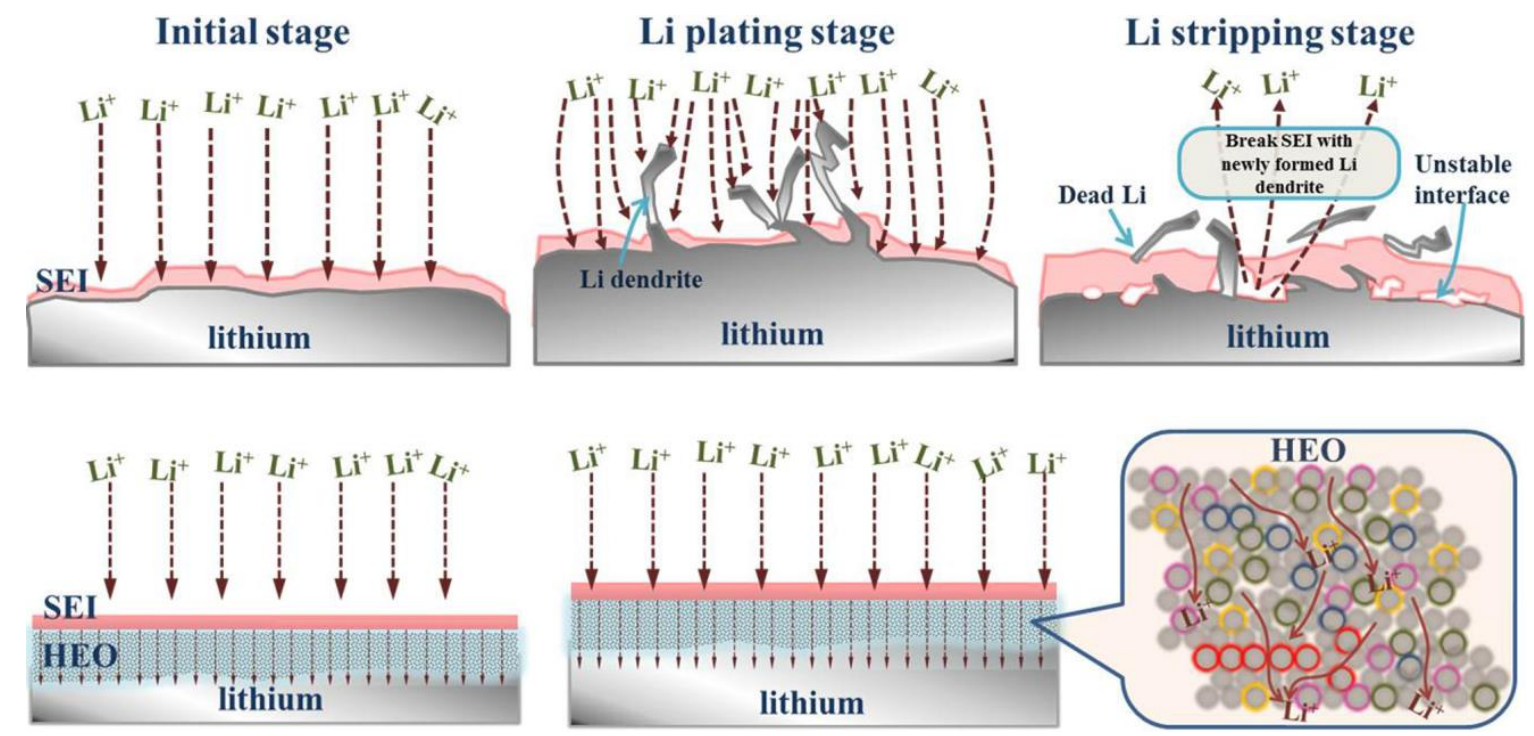

Figure 49 Schematic diagrams of the different Li anode structures. (a) Dendritic Li growth on pure lithium anode with uniform $\mathrm{Li}^{+}$flux and unstable SEI. (b) Modifying the $\mathrm{Li}$ foil with $\mathrm{Li}^{+}$ conducting high entropy oxide layer with uniform $\mathrm{Li}^{+}$flux and stable interface. 


\subsubsection{Material synthesis and film preparation}

Synthesis of high entropy oxides: All samples with nominal compositions ( $\mathrm{MgCoNiCuZn})_{1-}$ ${ }_{x} \mathrm{Li}_{\mathrm{x}} \mathrm{O}(x=0-0.5)$ were synthesized from stoichiometric mixtures of oxides and carbonate precursors: $\mathrm{MgO}$ (Alfa Aesar 99.95\%), CuO (Alfa Aesar 99.7\%), NiO (Strem Chemicals 4N), $\mathrm{Co}_{3} \mathrm{O}_{4}$ (Alfa Aesar 99.7\%), ZnO (Alfa Aesar 4N) and $\mathrm{Li}_{2} \mathrm{CO}_{3}$ (Alfa Aesar 99.998\%). Starting powders of these precursors with a total mass of $6 \mathrm{~g}$ were mixed using an agate mortar and pestle followed by ball milling using a PBM-04 Planetary Ball Mill (Micronano tools) at $260 \mathrm{rpm}$ during 12 hours. The resulting mixture was uniaxially pressed into disc-shaped pellets (16 $\mathrm{mm})$ under $300 \mathrm{MPa}$. These pellets were heated at $1050{ }^{\circ} \mathrm{C}$ in air during 12 hours in an alumina crucible with a heating rate of $5{ }^{\circ} \mathrm{C} \mathrm{min}^{-1}$ from room temperature to $1050{ }^{\circ} \mathrm{C}$ and then kept at $1050{ }^{\circ} \mathrm{C}$ for 12 hours followed by air quenching. This process can be repeated several times until a pure single phase can be obtained. The resultant products were mechanically crushed and ground for at least 1 hour until a uniform powder could be obtained.

Ionic conducting PEO-HEO film preparation: The PEO-HEO film on Li foil was prepared in the glove box. Typically, $0.4 \mathrm{~g}$ of the prepared $\mathrm{HEO}_{\mathrm{x}}$ particles was mixed with $0.1 \mathrm{~g}$ PEO and $0.1 \mathrm{~g}$ LiTFSI with acetonitrile $(\mathrm{ACN})$ as the solvent. After vigorously stirred to obtain a homogeneous slurry, the formed suspension was dropped onto the Li discs by syringe to ensure a uniform and comparative loading for each disc, and then dried in the glove box to form a PEOHEO hybrid film on the Li metal discs.

Coulombic efficiency measurements: To evaluate the advantages of HEOx for Coulombic efficiency during Li stripping and plating. Half cells were assembled by using bare $\mathrm{Cu}$ foil and $\mathrm{Li}_{0.5}$-coated $\mathrm{Cu}$ foil as working electrodes, $\mathrm{Li}$ foil as reference/counter electrodes. The cells were tested at various current densities $\left(0.5 \sim 2 \mathrm{~mA} \mathrm{~cm}^{-2}\right)$ to make a better comparison. 


\subsubsection{Morphology and structure characterization}

Figure 50 shows the XRD patterns of the series of compounds with nominal composition $(\mathrm{MgCoNiZnCu})_{1-\mathrm{x}} \mathrm{Li}_{\mathrm{x}} \mathrm{O}(\mathrm{x}=0$ to 0.5$)$. As it can be observed, all the compounds present a singlephase structure, which agrees well with the standard PDF card of rock-salt (ICSD-9866). The detailed information of the XRD patterns between $2 \theta=36^{\circ} \sim 44^{\circ}$ and $2 \theta=61^{\circ} \sim 64^{\circ}$ are presented in Figure $50 \mathrm{~b}$ and $\mathrm{c}$, respectively. It is clear that the major peaks shift to the direction of higher diffraction angles with the increasing amount of $\mathrm{Li}$ atoms in the compounds. This is because of the decreased interplanar crystal spacing after the Li substitution, as the diameter of $\mathrm{Li}$ atoms is much smaller than that of other transition metals. In addition, the $\mathrm{Li}^{+}$conductivity can be significantly improved by increasing the amount of substituted Li atoms. As summarized in Table 7 by Bérardan et al., ${ }^{[201]}$ the Li ionic conductivity almost improves 5 orders of magnitude when increasing $x$ to 0.33 compared with the original oxides without $\mathrm{Li}$ substitution at room temperature. Such high Li ionic conductivity of HEO thus is expected to enable an excellent electrochemical performance for stable Li metal anode applications.
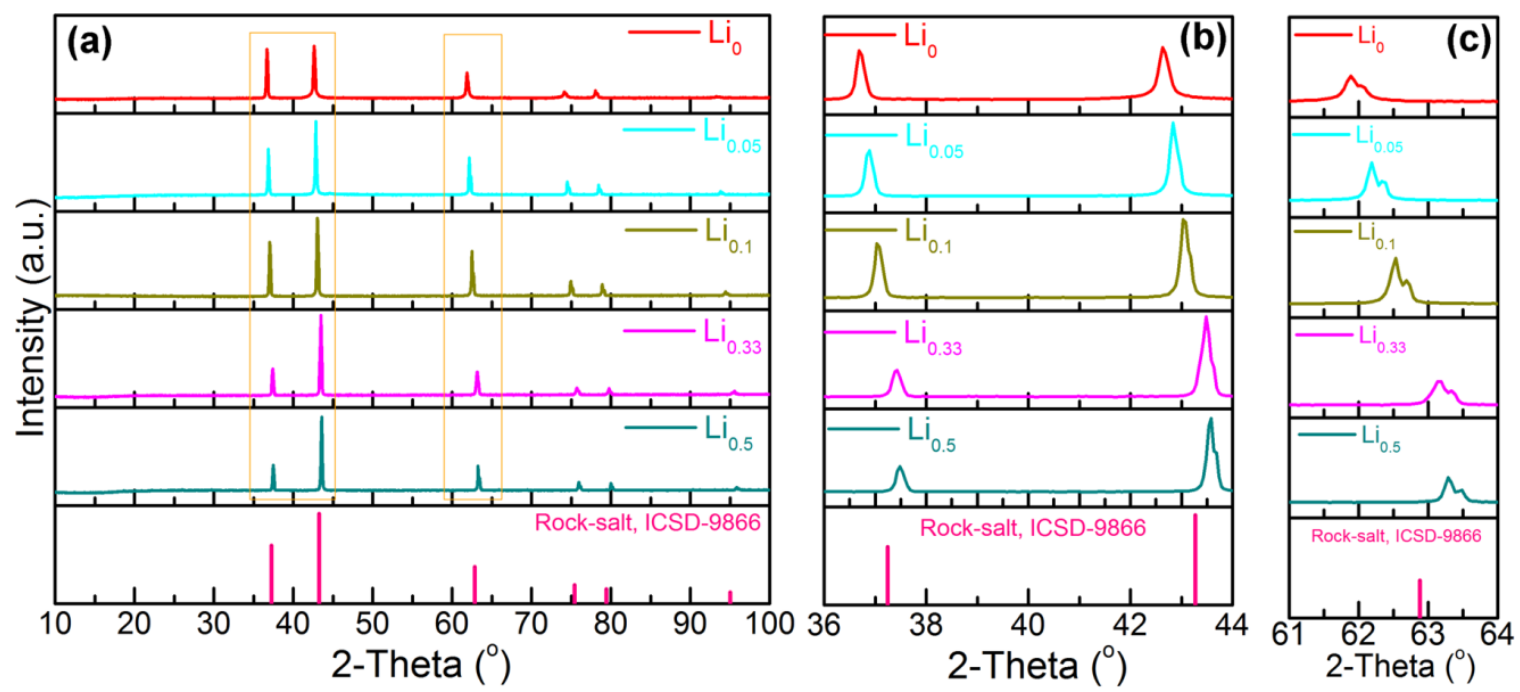

Figure 50 (a) XRD diffraction patterns of the series $(\mathrm{MgCoNiZnCu})_{1-\mathrm{x}} \mathrm{Li}_{\mathrm{x}} \mathrm{O}(\mathrm{x}=0$ to 0.5$)$, and the corresponding enlarged patterns at $2 \theta$ between $36^{\circ}$ and $44^{\circ}(\mathrm{b})$, and $61^{\circ}$ and $64^{\circ}$ (c). 
Table 7 Ionic conductivity for $\mathrm{HEO}_{\mathrm{x}}$ samples. ${ }^{[201]}$

\begin{tabular}{ccccccccc}
\hline $\mathbf{H E O}_{\mathbf{X}}$ & $\mathbf{x}=\mathbf{0}$ & $\mathbf{x}=\mathbf{0 . 0 2}$ & $\mathbf{x}=\mathbf{0 . 0 8}$ & $\mathbf{x}=\mathbf{0 . 1}$ & $\mathbf{x}=\mathbf{0 . 1 6}$ & $\mathbf{x}=\mathbf{0 . 2}$ & $\mathbf{x}=\mathbf{0 . 2 5}$ & $\mathbf{x}=\mathbf{0 . 3 3}$ \\
\hline $\boldsymbol{\sigma}\left(\mathbf{S ~ c m}^{-1}\right)$ & $3 \times 10^{-8}$ & $3 \times 10^{-8}$ & $1.5 \times 10^{-7}$ & $2 \times 10^{-7}$ & $8 \times 10^{-6}$ & $3 \times 10^{-4}$ & $3 \times 10^{-4}$ & $1 \times 10^{-3}$ \\
$\quad \mathbf{2 0} \mathbf{C}^{\mathbf{0}}$ & & & & & & & \\
$\boldsymbol{\sigma}\left(\mathbf{S ~ c m}^{-1}\right)$ & $1.5 \times 10^{-7}$ & $8 \times 10^{-7}$ & $6 \times 10^{-7}$ & $2 \times 10^{-6}$ & $1 \times 10^{-5}$ & $7 \times 10^{-4}$ & $1 \times 10^{-3}$ & $4 \times 10^{-3}$ \\
$\quad \mathbf{8 0}^{\mathbf{0}} \mathbf{C}$ & & & & & \\
\hline
\end{tabular}

To get a better insight into the charge compensation mechanism in these materials, an XPS study has been conducted. Figure 51 shows the XPS profiles of three typical HEO compounds, i.e., $\mathrm{Li}_{0}, \mathrm{Li}_{0.33}$, and $\mathrm{Li}_{0.5}$. All three samples show identical $\mathrm{Mg} 1 \mathrm{~s}, \mathrm{Co} 2 \mathrm{p}, \mathrm{Ni} 2 \mathrm{p}, \mathrm{Cu} 2 \mathrm{p}, \mathrm{Zn} 2 \mathrm{p} 3$ spectra as shown in the survey scan profiles (Figure 51a). Overall, the peaks of the metal elements shift to the lower binding energy after $\mathrm{Li}^{+}$substitution (Figure $51 \mathrm{~b}-\mathrm{f}$ ). The effect of $\mathrm{Li}^{+}$ substitution can directly be observed in the Li 1s spectra, for the doped samples, a Li 1s peak appeared at $54.7 \mathrm{eV}$ whose intensity increased with the amount of lithium (Figure 51f). Notable changes can be observed in the spectra of Co (Figure 51c) and $\mathrm{O}$ (Figure 51h). For the $\mathrm{Li}_{0}$ sample (undoped with Li), the XPS spectra for the Co $2 p$ peak is typical of a $\mathrm{Co}^{2+}$ state, with broad main lines $2 p_{3 / 2}$ and $2 p_{1 / 2}$ and with intense satellite peaks at $786.0 \mathrm{eV}$ and $803.2 \mathrm{eV}$. With increasing the $\mathrm{Li}^{+}$substitution, the main peaks at 780.2 and $796.5 \mathrm{eV}$, can be deconvoluted into two peaks, respectively, with a new satellite at $\sim 789 \mathrm{eV}$. This result evidently reveals the charge compensation upon $\mathrm{Li}^{+}$substitution. In addition, the XPS spectra for $\mathrm{O} 1 \mathrm{~s}$ also show great differences in these three samples $\left(\mathrm{Li}_{0}, \mathrm{Li}_{0.33}\right.$, and $\left.\mathrm{Li}_{0.5}\right)$. The main $\mathrm{O}$ 1s peak appeared at 529.6 $\mathrm{eV}$ assigned to $\mathrm{O}^{2-}$ of the lattice $(529.7 \mathrm{eV})$ in addition to a shoulder peak at $531.3 \mathrm{eV}$ whose intensity obviously increased with $\mathrm{Li}^{+}$substitution and which could be assigned to hydroxides or oxygen defects. Thus, the compensation mechanism should probably involve both the charge compensation of the metal ions and the formation of oxygen defects in the system. This also confirms that this HEO can be chemically versatile by simply changing the metal cations composition to enable expected electrochemical performance. 

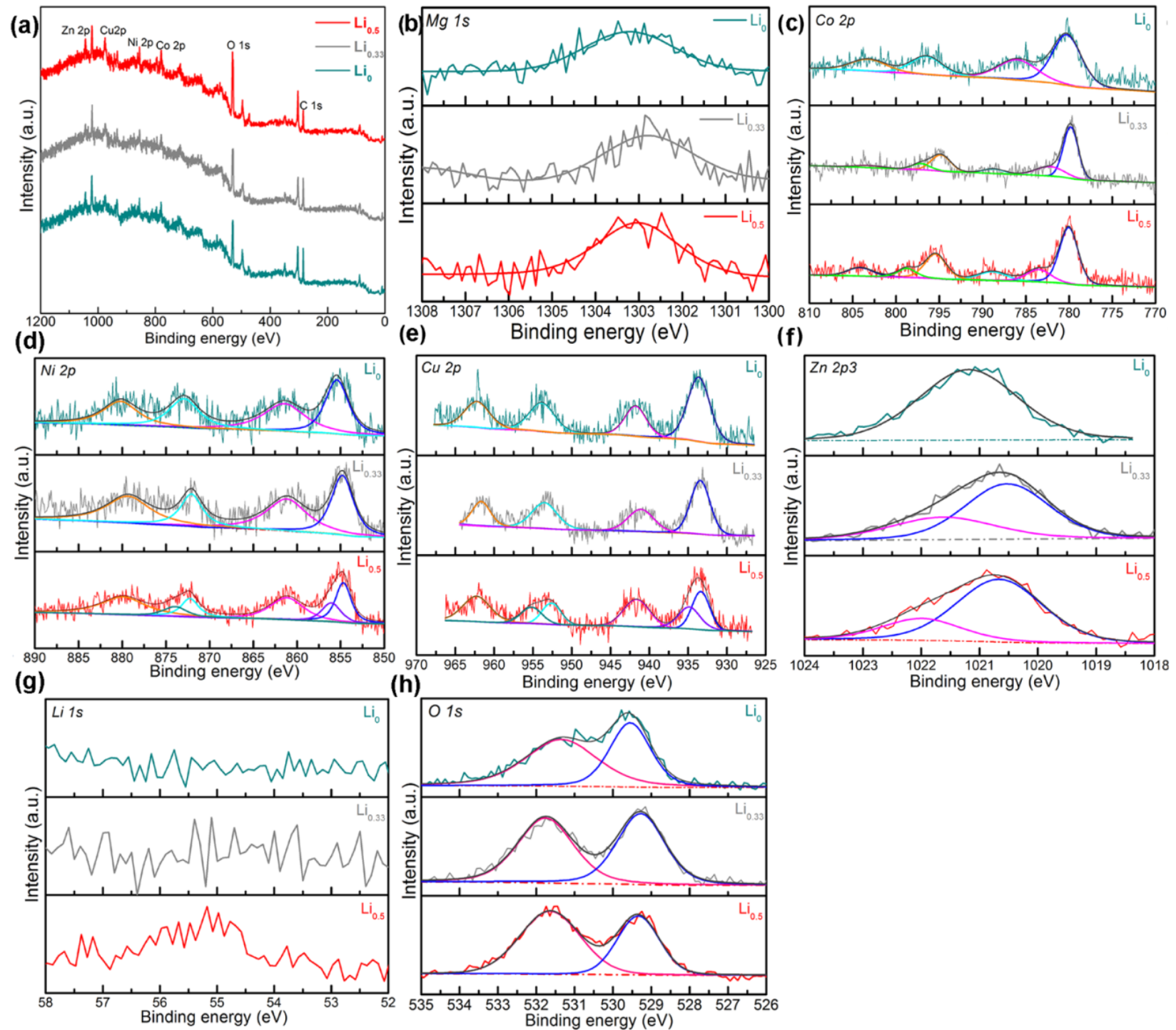

Figure 51 Comparison of XPS spectra for different $\mathrm{HEO}_{\mathrm{x}}$ including $\mathrm{Li}_{0}, \mathrm{Li}_{0.33}$, and $\mathrm{Li}_{0.5}$. (a) $\mathrm{Mg}$ 1s, (b) Co 2p, (c) Ni 2p, (d) Cu 2p, (e) Zn 2p3, and (f) Li 1s spectra.

The morphological characterizations of the $\mathrm{HEO}_{\mathrm{x}}$ compounds were investigated by SEM and the results are illustrated in Figure 52. All the compounds exhibit similar irregular particle morphologies with a very broad size distribution. The particles present a glassy, dense, and clear cleavage surface. Some particles with a laminar structured surface can be observed. It should be noticed that the size of particles can be optimized by increasing the grinding time or using a 
more efficient ball milling machine. Figure $52 \mathrm{~g}$ shows the high magnification surface morphology of the $\mathrm{Li}_{0.5}$ sample, which further demonstrates the dense and glassy nature of the particles. The corresponding EDX mapping reveals that all the elements, including $\mathrm{Mg}, \mathrm{Co}, \mathrm{Zn}$, $\mathrm{Cu}, \mathrm{Ni}$, are homogeneously distributed.

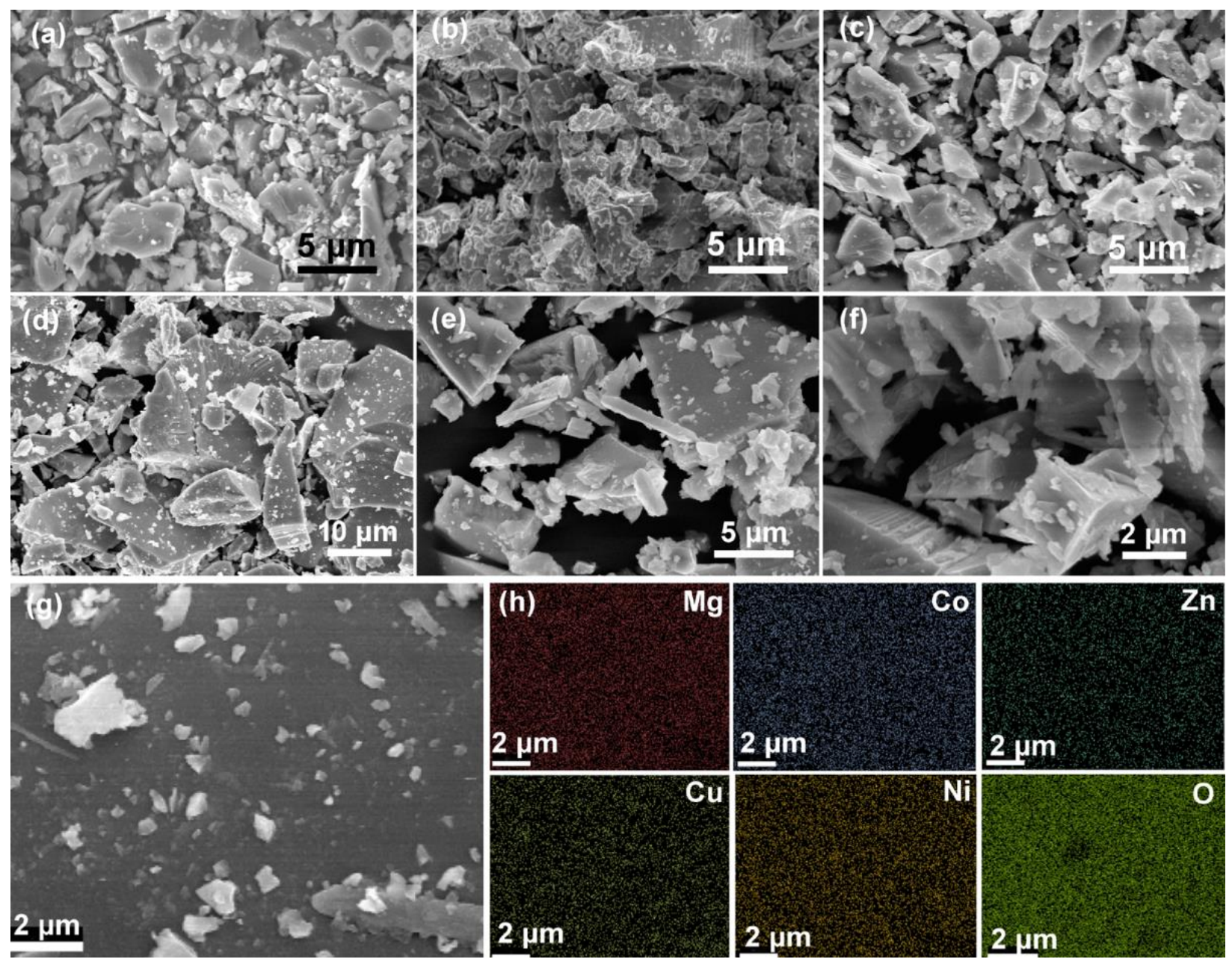

Figure 52 SEM images of synthesized $\mathrm{HEO}_{\mathrm{x}}$. (a) $\mathrm{x}=0$, (b) $\mathrm{x}=0.05$, (c) $\mathrm{x}=0.1$, (d) $\mathrm{x}=0.33$, (e, f) low and high magnification images of $\mathrm{Li}_{0.5}(\mathrm{x}=0.5)$. (g) The surface of $\mathrm{Li}_{0.5}$ particle and the corresponding EDX mapping (h).

When casting the PEO-HEO slurry onto the Li foil, the rock salt structure of the $\mathrm{HEO}_{\mathrm{x}}$ can still be maintained, indicating the high chemical stability of the synthesized $\mathrm{HEO}_{\mathrm{x}}$. As confirmed 
by the XRD pattern of PEO-Li $\mathrm{L}_{0.5}$ film on Li foil (Figure 53a), the peaks corresponding to PEO, HEO $\left(\mathrm{Li}_{0.5}\right)$, and $\mathrm{Li}$ foil substrate can be identified with only some small peaks corresponding to $\mathrm{LiOH} \cdot \mathrm{H}_{2} \mathrm{O}$ formed due to the exposure to the air during the XRD measurements. Figure 53b reveals that the thickness of the hybrid film coated on the Li foil is approximately $60 \sim 80 \mu \mathrm{m}$ with the oxide particles embedded in the PEO matrix. The surface morphology of the hybrid film in Figure 53c indicates that the PEO polymer can form a hermetic and conformal coating layer on the $\mathrm{Li}$ foil, with the HEO $\left(\mathrm{Li}_{0.5}\right)$ particles well dispersed in the polymer film. As further demonstrated by the elemental EDX mapping in Figure 53d-i. The elemental mappings for Mg, $\mathrm{Co}, \mathrm{Ni}, \mathrm{Cu}, \mathrm{Zn}$, and $\mathrm{O}$ confirm that the $(\mathrm{MgCoNiCuZn})_{0.5} \mathrm{Li}_{0.5} \mathrm{O}$ particles are homogeneously distributed in the polymer film, forming a robust coating layer on the Li metal. This hybrid film combined the flexibility of PEO and mechanical strength of HEO, therefore, can function as a robust protector, prevent the dendritic $\mathrm{Li}$ formation and penetration. 

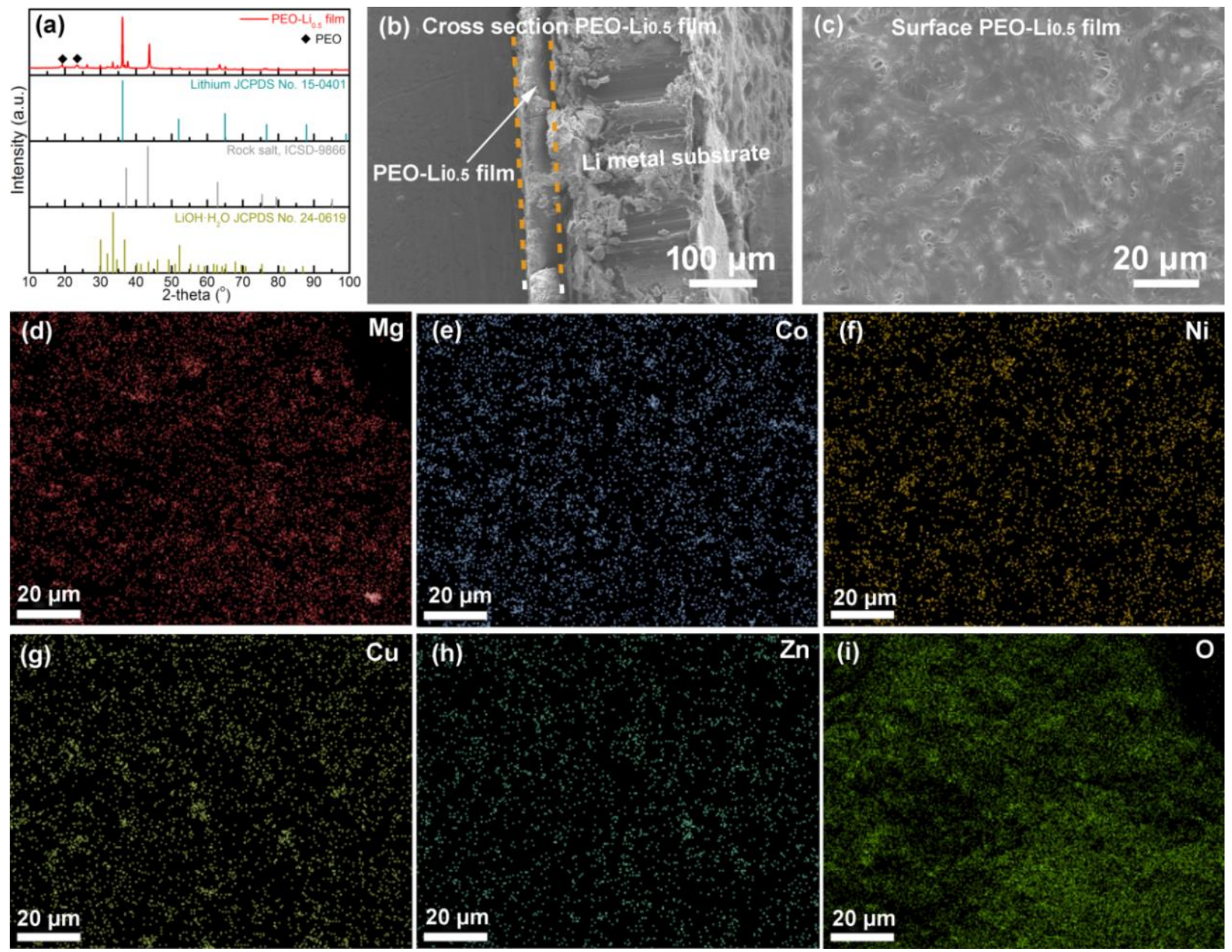

Figure 53 (a) XRD profile of PEO-Li ${ }_{0.5}$ hybrid film on the Li foil. (b) Cross-sectional SEM image showing the thickness of the PEO- $\mathrm{Li}_{0.5}$ film on the Li foil. (c) Surface morphology of the PEO- $\mathrm{Li}_{0.5}$ film with the corresponding elemental EDX mapping (d-i).

\subsubsection{Li stripping/plating behavior on planar $\mathrm{Cu}$ and $\mathrm{HEO}$ modified $\mathrm{Cu}$}

To demonstrate the advantages of the HEO protective layer on the Li foil, the Li deposition behaviors of planar $\mathrm{Cu}$ foil and $\mathrm{HEO}$ modified $\mathrm{Cu}$ foil $(\mathrm{HEO}-\mathrm{Cu})$ are investigated. As an example, $\mathrm{Li}_{0.5}$ was employed as the active material to prepare a protective coating layer on $\mathrm{Cu}$ foil. $\mathrm{Li}$ deposition/stripping cycles between $\mathrm{Cu}$ or $\mathrm{Li}_{0.5}-\mathrm{Cu}$ working electrodes and $\mathrm{Li}$ counter/reference electrodes are performed at various current densities to evaluate the Coulombic efficiency. The deposition capacity is set to be $1.0 \mathrm{mAh} \mathrm{cm}^{-2}$, and the cutoff potential for striping 
is set to be $1.0 \mathrm{~V}$. Figure 54 compares the potential profiles of cells at $0.5,1$, and $2 \mathrm{~mA} \mathrm{~cm}^{-2}$. It is clear that the voltage hysteresis of the $\mathrm{Cu} \| \mathrm{Li}$ half cells are much higher than that of $\mathrm{Li}_{0.5}-\mathrm{Cu} \| \mathrm{Li}$ half cells. The bare $\mathrm{Cu}$ electrodes show gradually decreased stripping capacities with cycling, resulting in the lower Coulombic efficiency. However, upon cycling, the $\mathrm{Li}_{0.5}-\mathrm{Cu}$ electrodes gradually become stable after a few cycles of activation, and can still overlap each other very well after 50 cycles, showing a relatively higher Coulombic efficiency. In addition, both electrodes show greater overpotential in the initial stage. The $\mathrm{Li}_{0.5}$ can contribute to the capacity of $\sim 0.2 \mathrm{mAh} \mathrm{cm}^{-2}$ in the first plating process, which is due to the electrochemical active property of HEO towards Li. After a few cycles, the HEO coating layer becomes stable and saturated with $\mathrm{Li}^{+}$. These results indicate the significant advantages of HEO coating as a stable artificial SEI layer to enable uniform $\mathrm{Li}^{+}$flux in the interface, stable Li stripping and plating electrochemistry. 

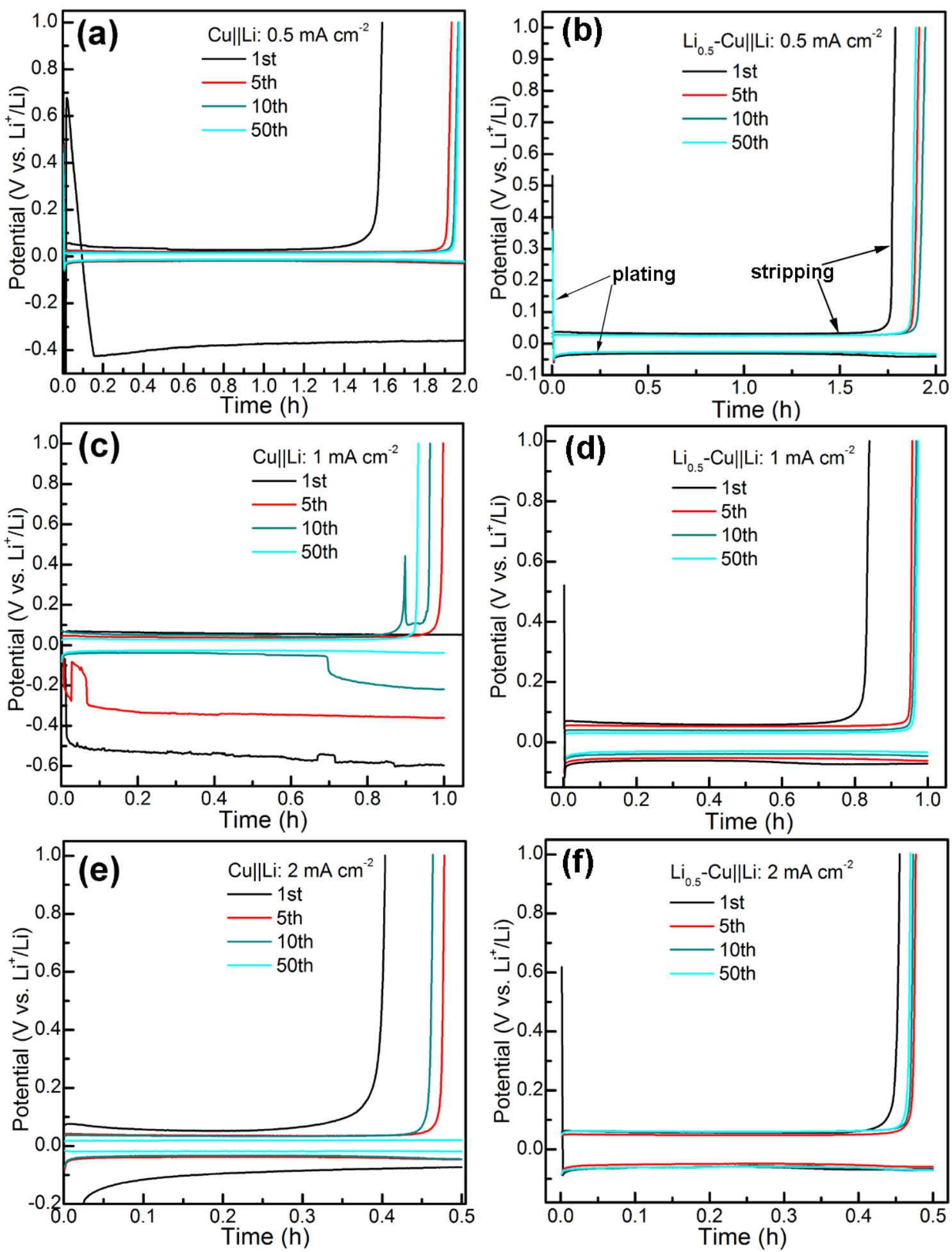

Figure 54 The typical voltage profiles of the $\mathrm{Cu} \| \mathrm{Li}(\mathrm{a}, \mathrm{c}, \mathrm{e})$ and $\mathrm{Li}_{0.5}-\mathrm{Cu} \| \mathrm{Li}(\mathrm{b}, \mathrm{d}, \mathrm{f})$ half cells at current densities of $0.5(a, b), 1(c, d)$, and $2(e, f) \mathrm{mA} \mathrm{cm}^{-2}$. 
To further demonstrate the advantages of PEO-HEO film as a protection layer on Li foil, the corresponding half cells after 50 cycles of stripping and plating were characterized by the EIS method and then disassembled in the glove box for SEM observation. Figure 55a and b show the surface and cross-sectional SEM images of bare $\mathrm{Cu}$ electrode after 50 cycles of Li stripping and plating. A lot of Li dendrites can be observed on the surface with a typical diameter of $\sim 2 \mu \mathrm{m}$. The thickness of the Li dendrite layer is around 15 20 $\mu \mathrm{m}$ (Figure 55b). The irregularly deposited Li on the surface cause unstable interface during the Li plating/ stripping and increased the interfacial area with thickened SEI film, thus resulting in the much higher charge transfer resistance $\left(\mathrm{R}_{\mathrm{ct}}\right)$, as confirmed by the EIS Nyquist curve in Figure $55 \mathrm{c}$. By contrast, the $\mathrm{Li}_{0.5^{-}}$ coated $\mathrm{Cu}$ foil can still maintain a clean and smooth surface (Figure 55d) without the formation of any large dendrites. The ion-conducting film allowed Li deposition only underneath the film to suppress the formation of $\mathrm{Li}$ dendrites (Figure 55e). The stabilized interface is further evidenced by the much smaller $\mathrm{R}_{\mathrm{ct}}$ in Figure 55f. A close observation of the $\mathrm{Cu}$ interface and $\mathrm{Li}_{0.5}$ particles are shown in Figure $55 \mathrm{~g}$ and $\mathrm{h}$, respectively, which confirms that Li preferably deposits beneath the PEO-HEO layer with the relatively smooth surface morphology of PEOHEO film. The large $\mathrm{Li}_{0.5}$ particles are wrapped with thick amorphous film, which should be ascribed to the SEI film. A line-scan profile was conducted across the interfaces. As shown in Figure 55i, three layers, including $\mathrm{Cu}$ substrate, $\mathrm{Li}$ deposited layer with electrolyte decomposition components, and PEO-HEO layer, are arranged very well. This result further demonstrates that PEO-HEO hybrid film can effectively suppress the formation of dendritic Li with strong mechanical and chemical stability. 

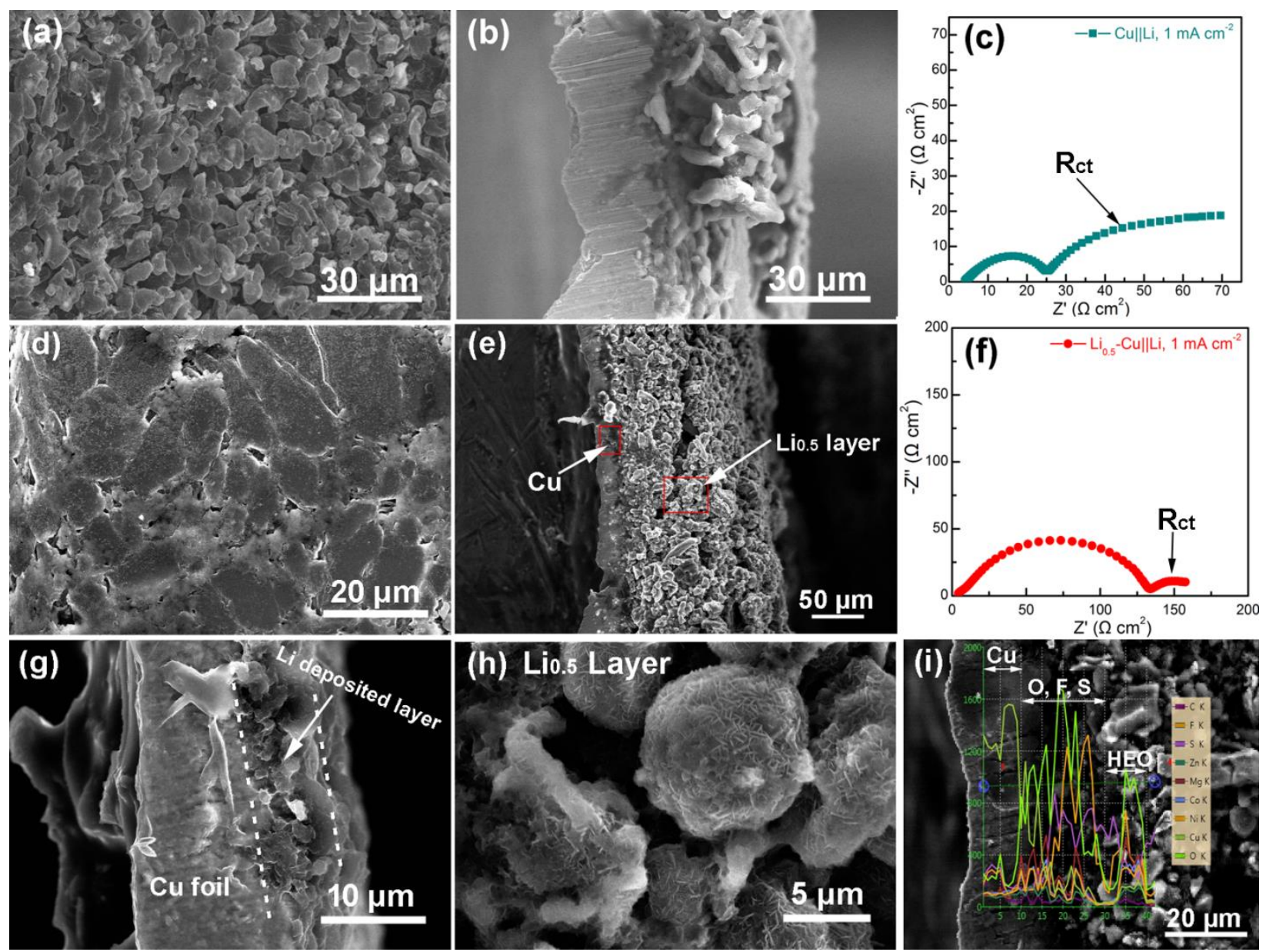

Figure 55 Surface (a, d) and cross-sectional (b, e) SEM images of bare $\mathrm{Cu}$ foil $(\mathrm{a}, \mathrm{b})$ and $\mathrm{Li}_{0.5^{-}}$ $\mathrm{Cu}(\mathrm{d}, \mathrm{e})$ after 50 cycles of stripping and plating with corresponding EIS Nyquist curves (c, f). The high magnification SEM images of corresponding Cu layer (g) and HEO layer (h), (i) Linescan of the $\mathrm{HEO}$ coated $\mathrm{Cu}$ electrode after 50 cycles of Li stripping and plating.

\subsubsection{Electrochemical performance of symmetric cells}

Symmetrical cells were assembled for testing to further verify that the PEO-HEO hybrid film can enable stable Li stripping/plating electrochemistry by regulating the $\mathrm{Li}^{+}$flux on the interface. As shown in Figure 56, the cycle performance of $\mathrm{Li}|| \mathrm{Li}, \mathrm{Li}_{0.33}|| \mathrm{Li}_{0.33}$, and $\mathrm{Li}_{0.5}|| \mathrm{Li}_{0.5}$ symmetric cells at different current densities are compared. In all cases, bare Li metal symmetric cells show significantly increased polarization and fluctuating voltages, while HEO based symmetric cells 
can sustain much stable voltage hysteresis. The Li $\| \mathrm{Li}$ symmetric cells exhibit short circuit only after $60 \mathrm{~h}, 40 \mathrm{~h}$, and $10 \mathrm{~h}$ at the current densities of $0.5,1$, and $2 \mathrm{~mA} \mathrm{~cm}^{-2}$, respectively. Overall, the $\mathrm{Li}$ anode modified by the $\mathrm{PEO}-\mathrm{HEO}_{\mathrm{x}}$ ion-conducting film can extend the cycle life to $\sim 1000$ hours (or more) without any large fluctuating voltages and short circuits. Some cells show initially activation process, but the voltage polarization gradually decreased and stabilized to $\sim 50$ $\mathrm{mV}$ when cycled at 0.5 and $1 \mathrm{~mA} \mathrm{~cm}{ }^{-2}$. Even at $2 \mathrm{~mA} \mathrm{~cm}$ (Figure 56c), the $\mathrm{Li}_{0.33} \| \mathrm{Li}_{0.33}$ symmetric cell can cycle stably after $\sim 10 \mathrm{~h}$ activation with voltage hysteresis gradually stabilized at $\sim 50 \mathrm{mV}$ during the $\sim 970 \mathrm{~h}$ of cycling. For $\mathrm{Li}_{0.5}|| \mathrm{Li}_{0.5}$ symmetric cell at $2 \mathrm{~mA} \mathrm{~cm}{ }^{-2}$, it can also maintain small voltage hysteresis during the first 500 hours. Although the slightly increased polarization can be observed in the following cycling, it can still keep stable Li stripping and plating electrochemistry without any short circuit. Basically, the Li anode modified by the PEOgarnet ion-conducting layer initially exhibited similar voltage polarization, but the voltage polarization gradually decreased and stabilized to $\sim 15 \mathrm{mV}$. Throughout cycling for $1,000 \mathrm{~h}$, the hybrid membrane-protected Li metal anode exhibited excellent cycling stability, in contrast to the bare $\mathrm{Li}$, which showed unstable plating/stripping voltages and an increasing voltage hysteresis.

To further evaluate the deep charge-discharge properties of PEO-HEO hybrid film-coated Li metal anode, the symmetric cells were cycled at $1 \mathrm{~mA} \mathrm{~cm}{ }^{-2}$ with deep stripping and plating capacity of $5 \mathrm{mAh} \mathrm{cm}{ }^{-2}$. Starting from a few cycles of the activation process, the $\mathrm{Li}_{0.5} \| \mathrm{Li}_{0.5}$ symmetric cell can deliver a stable and long-term life up to $400 \mathrm{~h}$ with low voltage hysteresis as illustrated in Figure 57, showing a significantly improved Li stripping and plating electrochemistry. With such excellent cycle stability and effective protection against the formation of dendritic Li, the PEO-HEO hybrid film-coated Li metal anodes are showing the 
great promising application in the Li metal-based batteries with high energy density and long cycle life.
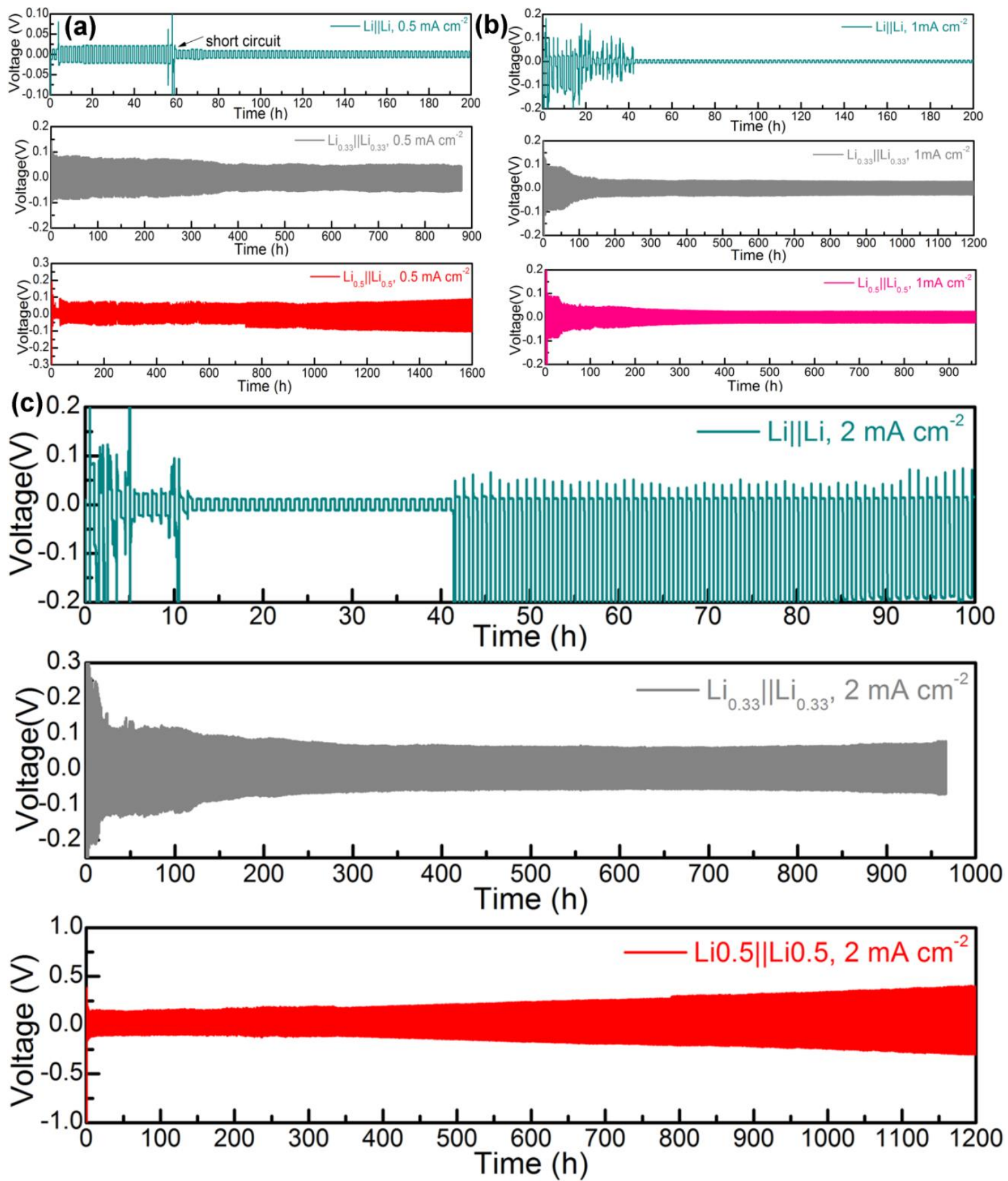

Figure 56 Long-term cycle stability of symmetrical cells for $\mathrm{Li}|| \operatorname{Li}, \mathrm{Li}_{0.33}|| \mathrm{Li}_{0.33}$, and $\mathrm{Li}_{0.5}|| \mathrm{Li}_{0.5}$ symmetric cells 0.5 (a), 1 (b), and 2 (c) $\mathrm{mA} \mathrm{cm}^{-2}$ with constant capacity of $1 \mathrm{mAh} \mathrm{cm}^{-2}$ for $100 \mathrm{~h}$. 


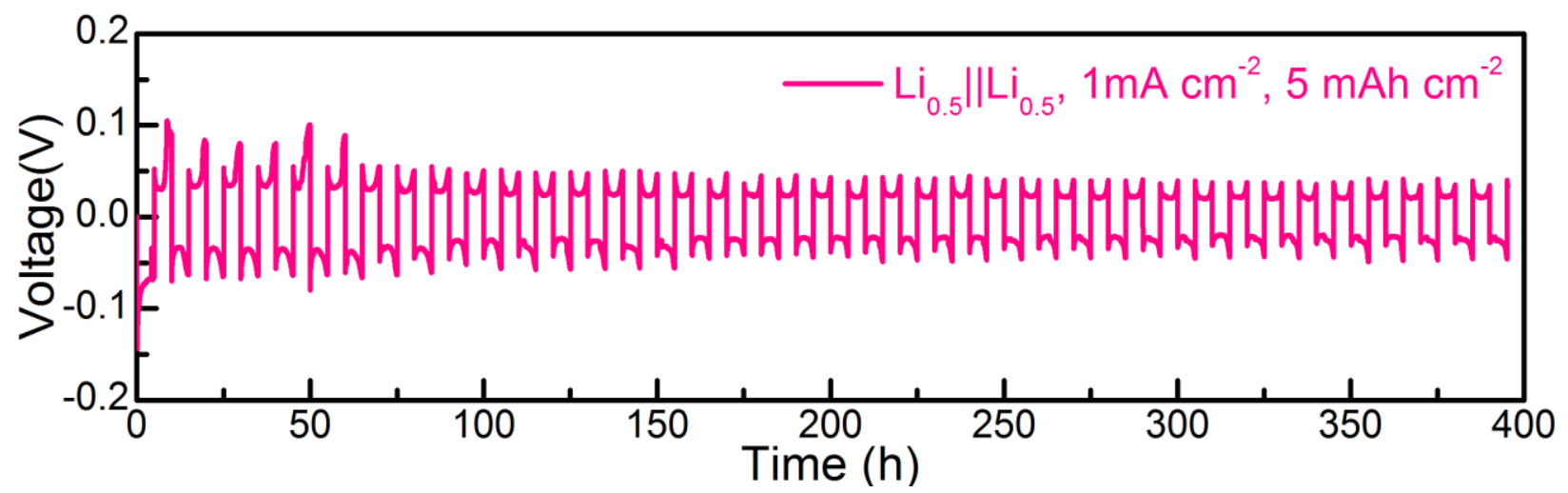

Figure 57 Galvanostatic cycling performance of $\mathrm{Li}_{0.5}|| \mathrm{Li}_{0.5}$ symmetric cell at a stripping/plating capacity of $5 \mathrm{mAh} \mathrm{cm}^{-2}$ at $1 \mathrm{~mA} \mathrm{~cm}{ }^{-2}$.

\subsection{D Conductive Hairy Foam as Stable Lithium Deposition Medium}

\subsubsection{Introduction}

Besides the ex-situ formed "artificial SEI layer" to address the challenges for Li metal anodes, another widely used method is employing a 3D conductive host for Li deposition. It has been confirmed that the conductive host with a 3D skeleton can effectively reduce the local current density and enable a uniformly distributed Li deposition due to the large specific surface area. Based on Newman's models, the growth of dendritic Li is much related to the distribution of Liion concentration and local potential. ${ }^{[125]} 3 \mathrm{D}$ porous and conductive host electrodes can promote the fast $\mathrm{Li}^{+}$diffusion and regulate the local current density distribution, thus can enable a uniform Li deposition behavior. In addition, the 3D porous skeleton with the large specific surface area can help to accommodate volume variation of Li metal, compensate local supply, and more importantly, reduce effective local current density for more homogeneous Li nucleation and growth. However, the 3D foam cannot guarantee a uniform Li deposition due to the large Li nucleation overpotential. Yan et al. investigated the Li nucleation overpotential on a list of substrate materials $(\mathrm{Au}, \mathrm{Si}, \mathrm{Ni}, \mathrm{Cu}$, etc.) and proposed a heterogeneous seeded growth 
method by using Au nanoseeds to selectively nucleate and grow Li metal inside the carbon nanoshells. ${ }^{[202]}$ Following this concept, various 3D frameworks with hierarchical structure have been designed to improve the electrochemical stability of Li metal anode. Yang et al. demonstrated that the $3 \mathrm{D}$ porous $\mathrm{Cu}$ electrode with a submicron skeleton can remarkably suppress the growth of dendritic Li compared with the planar $\mathrm{Cu}$ current collector. ${ }^{[127]}$ The submicron $\mathrm{Cu}$ fibers function as the charge centers and nucleation sites to induce the uniform electric field and homogeneous nucleation of $\mathrm{Li}$. Lu et al. modified the Ni foam by coating a graphitic carbon nitride $\left(\mathrm{g}_{-} \mathrm{C}_{3} \mathrm{~N}_{4}\right)$ layer. ${ }^{[203]}$ The lithiophilic g- $\mathrm{C}_{3} \mathrm{~N}_{4}$ induced a uniform microelectric field and numerous Li nuclei, thus effectively regulating the following Li deposition to realize a remarkable electrochemical performance of dendrite-free $\mathrm{Li}$ anode with high Coulombic efficiency and long lifespan. Similarly, $\mathrm{Wu}$ and co-workers synthesized a lithiophilic $\mathrm{Cu}-\mathrm{CuO}-\mathrm{Ni}$ hybrid structure as Li host. ${ }^{[204]}$ The uniform $\mathrm{Li}^{+}$flux and Li nucleation induced by the nanowire arrays on the $\mathrm{Ni}$ foam enable the effective suppression of dendritic $\mathrm{Li}$ growth, resulting in high Coulombic efficiency and stable Li stripping/plating in a symmetric cell.

Based on the aforementioned results, it is promising to design a $\mathrm{Li}$ plating matrix with low $\mathrm{Li}$ nucleation overpotential and high surface area to develop a stable Li metal anodes. Following this idea, the carbon nanofibers (CNF) grown on Ni foam- "hairy foam" (CNF@Ni) has been employed both as Li host and current collector to enable uniform Li deposition. The flourishing grown CNF significantly increases the effective surface area with correspondingly reduced local current density, and therefore resulting in the homogeneous Li plating on the CNF@Ni foam. In addition, the carbon matrix, as well as the randomly distributed metal nanoparticles, acts as the nucleus and Li crystallized and grown along/outside the surface of CNF or metal nanoparticles. This heterogeneous nucleation process lowers the Li nucleation overpotential and induces more 
Li nucleation sites to promote uniform and smooth Li deposition and growth. Moreover, the void space between the interwoven $\mathrm{CNFs}$ as well as the open pores of $\mathrm{Ni}$ foam substrate can effectively accommodate the large volume change to keep the stability of the electrode. As a result, a stable Li stripping/plating can be achieved with Coulombic efficiency closed to $\sim 100 \%$. The ultralong lifespan of $\sim 1000 \mathrm{~h}$ at $0.5 \mathrm{C}\left(1 \mathrm{C}=1 \mathrm{~mA} \mathrm{~cm}{ }^{-2}\right)$ with an areal capacity of $1.0 \mathrm{mAh}$ $\mathrm{cm}^{-2}$ can be achieved in the CNF@Ni-Li symmetrical cell. Even at $5 \mathrm{C}$, stable cycle performance with relatively low voltage hysteresis can be maintained for $300 \mathrm{~h}$.

\subsubsection{Preparation of CNF@Ni hairy foam and material characterization}

The hairy foam (CNF@Ni) with carbon nanofibers grown on the 3D Ni foam skeleton was obtained by a nano metal particle catalytic process during chemical vapor deposition (CVD), as shown in Chapter 5. Simply, Ni form discs were firstly immersed into the $80 \mathrm{~mL}$ solution containing $0.02 \mathrm{~mol} \mathrm{~L}^{-1}$ of $\mathrm{FeSO}_{4} \cdot 7 \mathrm{H}_{2} \mathrm{O}, 0.042 \mathrm{~mol} \mathrm{~L}^{-1}$ of urea, and $6.94 \times 10^{-4} \mathrm{~mol} \mathrm{~L}^{-1}$ of sodium lauryl sulfate (SDS). The subsequent hydrothermal treatment at $100{ }^{\circ} \mathrm{C}$ for $12 \mathrm{~h}$ enabled a thin oxide layer grown on the surface of Ni foam discs. During the following CVD process under 5\% $\mathrm{H}_{2} / \mathrm{Ar}$ atmosphere, the oxide layer could be reduced to nanosized metal particles and further catalyze the carbon nanofibers growth due to the pyrolysis of toluene at $800{ }^{\circ} \mathrm{C}$. The CVD deposition time can be varied to adjust the carbon loading on the Ni foam. When used as lithium host (anode), the deposition time is within 1 hour to minimize the capacity contributed by the lithiation of graphitized carbon. The corresponding areal loading of carbon on $\mathrm{Ni}$ foam was controlled around 6-8 $\mathrm{mg} \mathrm{cm}^{-2}$. 


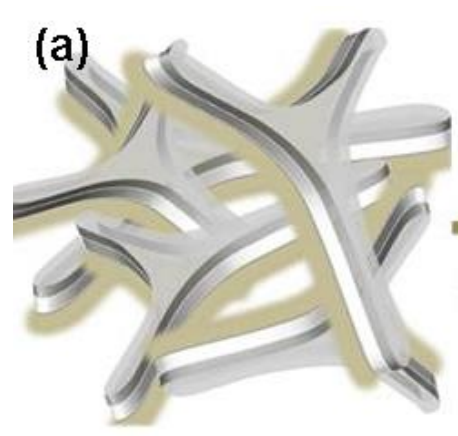

Pristine Ni foam

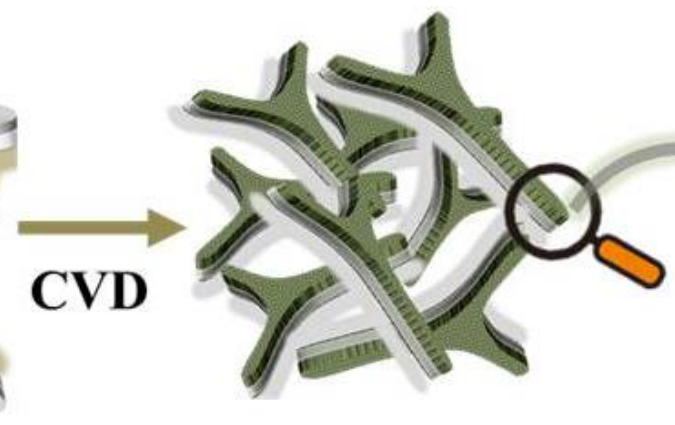

Hairy Foam

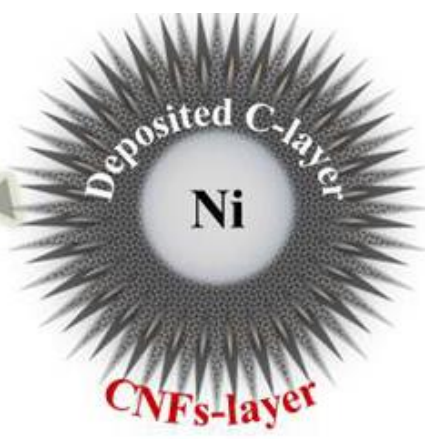

Cross-section

(b)

Li dendrite growth

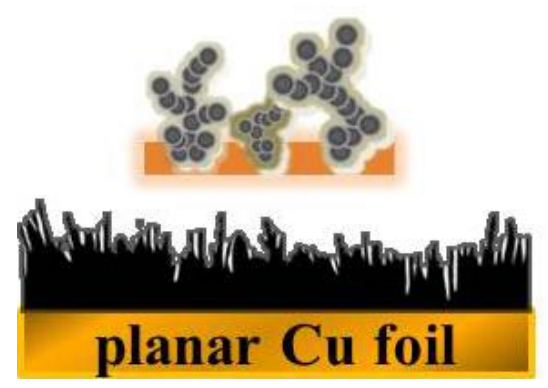

\section{Li dendrite growth}

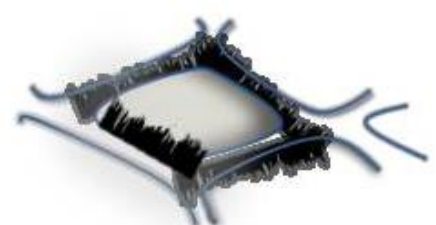

3D Ni foam
Li deposited along CNFs

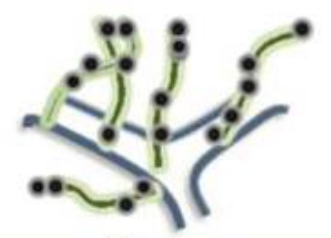

3DCNF@Nifoam-

Figure 58 (a) Schemed synthesis process of CNF@Ni foam, (b) comparison of Li deposition behavior for different host electrodes: planar Cu,3D Ni foam, and 3D CNF@Ni foam.

Figure 59a shows the XRD pattern of the as-prepared CNF@Ni foam. The dominant peaks coincide well with the standard PDF cards of Ni (JCPDS No. 04-0850), resulting from the metallic Ni foam substrate. In addition, a small peak centred at $\sim 26^{\circ}$ can be ascribed to the graphitic carbon, which agrees well with the standard PDF cards of graphite (JCPDS No. 411487). To further reveal the structural characteristics of graphitic carbon on the Ni foam, the Raman spectrum was recorded as shown in Figure 59b. Two prominent peaks at 1347 and 1582 $\mathrm{cm}^{-1}$ corresponding to the $\mathrm{D}$ (disordered carbon) and $\mathrm{G}$ (graphitic carbon) bands are welldocumented in the previous literature. The relatively lower $\mathrm{ID} / \mathrm{IG}$ ratio $(\mathrm{ID} / \mathrm{IG}=0.785)$ indicates that the carbon matrix is highly graphited in agreement with the XRD result in Figure 59a. The C 
1s XPS in Figure 59c further illustrates the chemical structure of the "hair" on the surface of Ni foam. The C 1s XPS spectrum can be deconvoluted into four peaks. The typical peak at 284.8 and $286.6 \mathrm{eV}$ can be ascribed to sp2 hybridized carbon $(\mathrm{C}-\mathrm{C} / \mathrm{C}=\mathrm{C})$ and $\mathrm{C}-\mathrm{O}$ species, respectively. The peak at $285.5 \mathrm{eV}$ can be indexed to $\mathrm{C}-\mathrm{N}$ bonds. ${ }^{[205]} \mathrm{A}$ small shoulder peak at $283.6 \mathrm{eV}$ corresponds to the Ni-C bond resulting from the $\mathrm{Ni}_{3} \mathrm{C}$ formed during the CVD process. The morphologies of Ni foam substrate and CNF@Ni were characterized by SEM. Compared with the smooth surface of pristine $\mathrm{Ni}$ foam, the $\mathrm{Ni}$ foam can be coated with numerous carbon nanofibers as well as uniformly distributed metal nanoparticles after the CVD process (Figure 59d-f). The 3D structure of the Ni foam framework can be well maintained after the CVD process with a pore size of 200-250 $\mu \mathrm{m}$ (Figure 59d). The higher magnified SEM image in Figure 59e reveals the intercrossed CNFs terminated with metal nanoparticles (as circled in Figure 59e) on the surface. The flourishing CNFs are twisted and smooth with the diameter of 50-60 nm, which are hoped to provide sufficient active sites for lithium deposition and to regulate the local distribution of the electrical field by increasing the effective surface area. The cross-sectional image in Figure 59f further reveals the hierarchical architecture of CNF@Ni foam with the fluff-like CNFs sprouted out of the deposited carbon layer. Both EDX mappings of the surface (Figure 60) and cross-section (Figure 61) of CNF@Ni demonstrate the homogeneous distribution of $\mathrm{Ni}$ and $\mathrm{C}$ on the interface. 

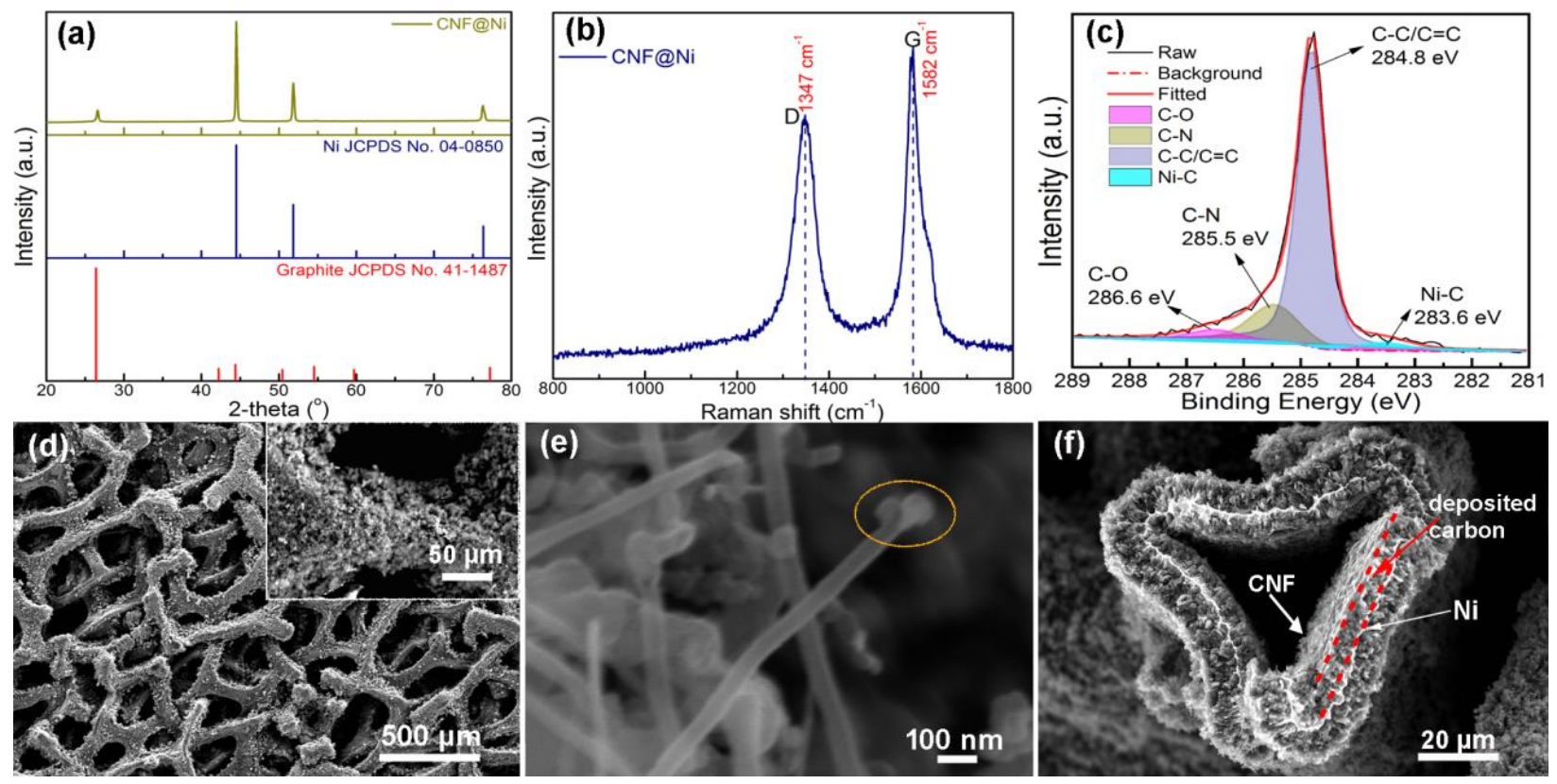

Figure 59 (a) XRD pattern of CNF@Ni and standard XRD card of Ni and graphite, Raman (b), and C 1s XPS (c) spectra of CNF@Ni. SEM images of CNF@Ni at low (d) and high (e) magnification with the corresponding cross-sectional image (f).

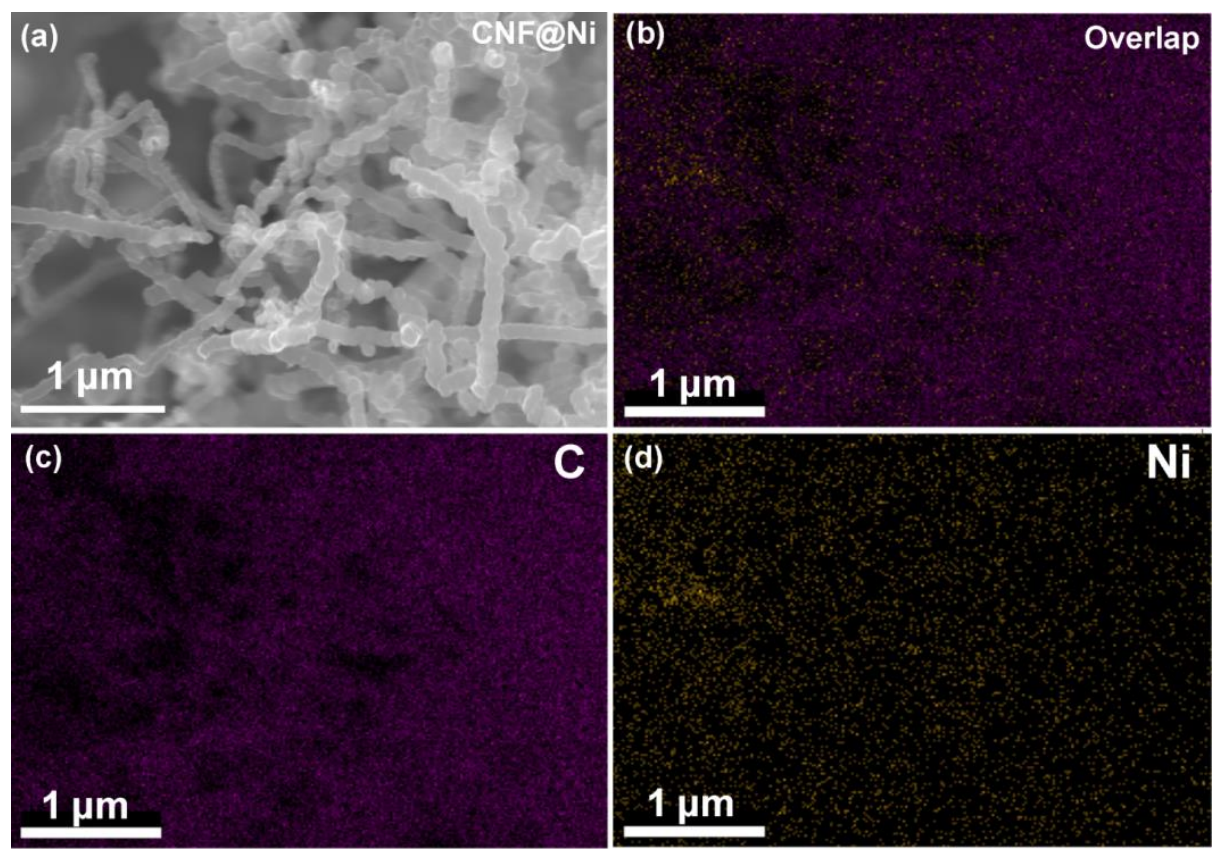

Figure 60 Surface SEM image of CNF@Ni and corresponding elemental maps of overlap (b), C (c), and $\mathrm{Ni}(\mathrm{d})$. 


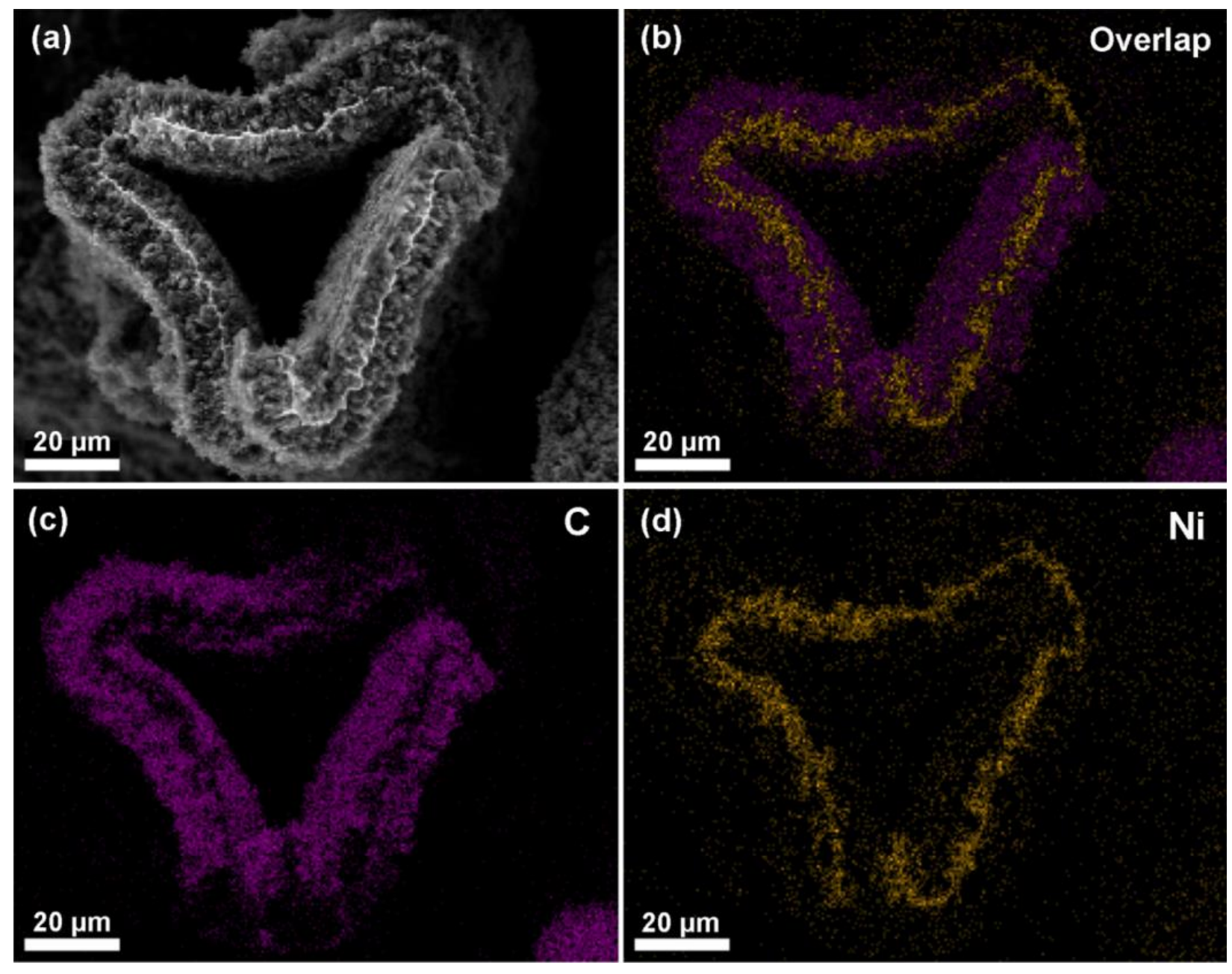

Figure 61 Cross-section SEM image of CNF@Ni and corresponding elemental maps of overlap (b), C (c), and $\mathrm{Ni}(\mathrm{d})$.

\subsubsection{Li stripping/plating behavior on planar $\mathrm{Cu}, 3 \mathrm{D}$ Ni foam, and CNF@Ni foam}

To illustrate the advantages of the CNF@Ni foam as lithium host, the Li deposition behaviors of planar $\mathrm{Cu}$ foil, pure Ni foam, and CNF@Ni hairy foam are investigated. Figure 62 collects the surface morphology evolution of $\mathrm{Li}$ deposition on the planar $\mathrm{Cu}$ at different stages. Compared with the pristine planar $\mathrm{Cu}$ (Figure 62a), no obvious dendrites can be observed before Li plating capacity reaches to $0.6 \mathrm{mAh} \mathrm{cm}^{-2}$. Only a small $\mathrm{Li}$ nucleus attached to the $\mathrm{Cu}$ foil can be observed (Figure $62 \mathrm{~b}$ and c). With the plating capacity increasing to $1 \mathrm{mAh} \mathrm{cm}^{-2}$ (Figure $62 \mathrm{~d}$ ), 
the lithium dendrites grow significantly. The numerous and randomly oriented Li dendrites finally spread to the whole surface of $\mathrm{Cu}$ foil. Upon stripping gradually to $1 \mathrm{mAh} \mathrm{cm}^{-2}$ (Figure 62g), large Li dendrites clusters dissolved with small Li particles remained on the surface. These "dead Li" significantly lower the Coulombic efficiency during the repeated charge/discharge process. Similarly, pure Ni foam also shows numerous Li dendrites grown during the Li plating process. As the Li plating capacity increases to $1 \mathrm{mAh} \mathrm{cm}^{-2}$ (Figure 63d), the dendrites can almost completely cover the surface of $\mathrm{Ni}$ foam as well as the pores. However, due to the large specific surface area with 3D conductive network of $\mathrm{Ni}$ foam, the Li dendrites can be effectively stripped from the Ni foam substrate (Figure 63g), resulting in a much clearer surface than that of planar $\mathrm{Cu}$ (Figure 62g). Although exaggerated dendrites formed during the Li plating process (Figure 63h), only a small amount of "dead Li" can be observed after a complete stripping at 1.0 $\mathrm{V}$ (Figure 63g and i). The Li stripping/plating behavior above demonstrate that both planar $\mathrm{Cu}$ and 3D Ni foam cannot suppress the dendrite formation due to the similar "local surface". However, a porous host with a 3D conductive network structure can effectively accommodate the volume change; promote the stripping efficiency, and thus increasing the cycle stability and Coulombic efficiency. 

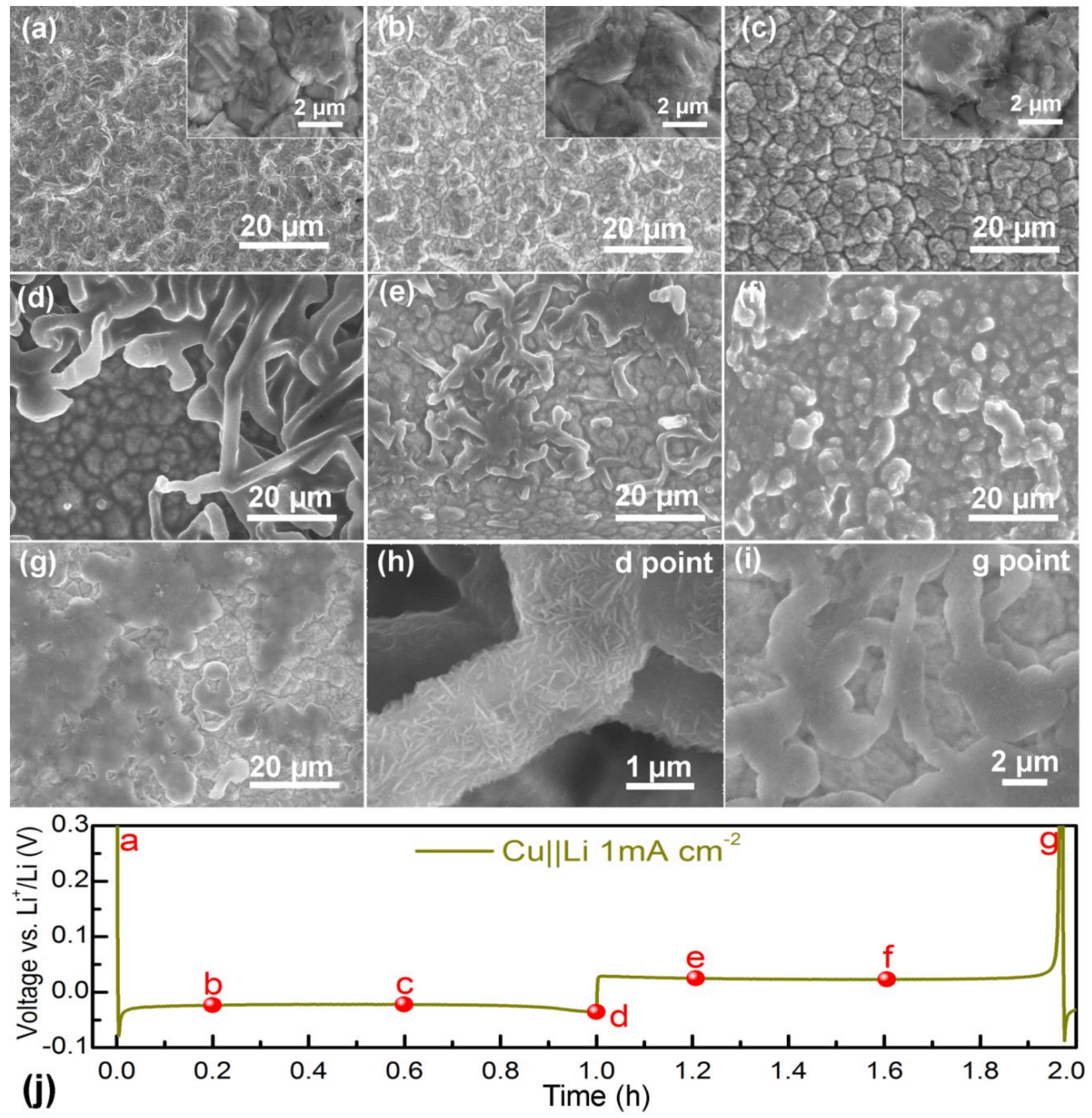

Figure 62 Morphological evolution of Li plating/stripping on planar $\mathrm{Cu}$, including pristine $\mathrm{Cu}$

(a), and after plating at 0.2 (b), 0.6 (c), $1 \mathrm{mAh} \mathrm{cm}^{-2}$ (d) of $\mathrm{Li}$ onto $\mathrm{Cu}$, and after stripping at 0.2 (e), 0.6 (f), $1 \mathrm{mAh} \mathrm{cm}^{-2}$ (g) of Li from Cu substrate. High magnification SEM images of d point (h) and g point (i). (j) Corresponding discharge/charge voltage profile of $\mathrm{Cu} \| \mathrm{Li}$ half-cell. 


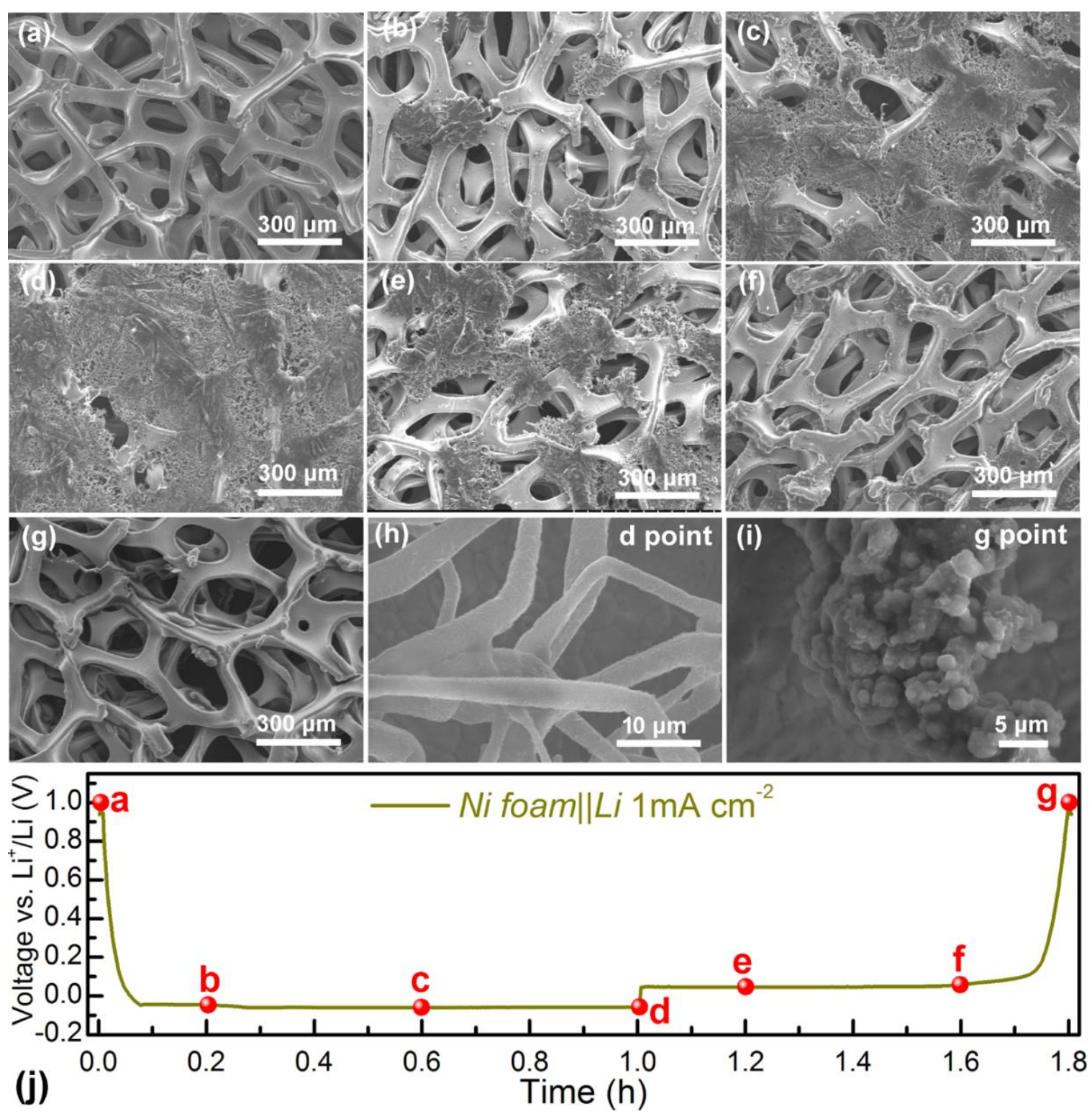

Figure 63 Morphological evolution of Li plating/stripping on pure Ni foam, including pristine Ni (a), and after plating at $0.2(\mathrm{~b}), 0.6(\mathrm{c}), 1 \mathrm{mAh} \mathrm{cm}^{-2}$ (d) of Li onto the Ni foam, and after stripping at $0.2(\mathrm{e}), 0.6$ (f), $1 \mathrm{mAh} \mathrm{cm}^{-2}$ (g) of Li from the Ni substrate. High magnification SEM images of d point (h) and g point (i). (j) Corresponding discharge/charge voltage profile of Ni||Li half-cell.

Remarkably, the Li stripping/plating on CNF@Ni shows a totally different morphological evolution. As graphitic carbon can be lithiated to consume lithium during the discharge process. To minimize the capacity contribution from the lithiated carbon, CNF@Ni foam discs with 
carbon loading within $10 \mathrm{mg} \mathrm{cm}^{-2}$ were selected as Li host to limit the capacity contribution from the lithiated carbon. Typically, when $\mathrm{Li}$ is plated with a constant current density of $1 \mathrm{~mA} \mathrm{~cm}^{-2}$ at cycling capacity of $1 \mathrm{mAh} \mathrm{cm}^{-2}$, no dendritic Li forms on the surface or in the pores as seen in Figure 64. At Li plating capacity of $0.2 \mathrm{mAh} \mathrm{cm}^{-2}$, the surface morphology of CNF@Ni foam shows no obvious change compared with the pristine CNF@Ni, however, the SEI begins to form based on the SEM images in Figure 64a and b. This is mainly due to the lithiated graphitic carbon rather than Li deposition at this initial stage. As the plating capacity increasing to $1 \mathrm{mAh}$ $\mathrm{cm}^{-2}$, there is still no obvious Li dendrites formed on the surface or in the open pores (Figure 64d). The increased volume results in the swollen Ni ligaments and the large amount SEI. As confirmed in the EDX spectrum and element mapping in Figure 65, respectively. The O, F, and S-containing species result from the decomposition of electrolyte to form the SEI layer. Compared with the smooth and clean CNFs of pristine CNF@Ni host (Figure 59e), the high magnified SEM image reveals that Li particles uniformly deposit along the carbon nanofibers (Figure 64h) without any significant aggregation. During the following stripping to $1.0 \mathrm{~V}$, the $\mathrm{Li}$ particles gradually dissolved with the rehabilitated clean and relatively smooth surface of CNFs, as illustrated in Figure 64g. In addition, the 3D skeleton of CNF@Ni foam can be wellmaintained after Li -lithiation/deposition, suggesting excellent structural stability and flexibility of the CNF@Ni in accommodating the volume change and increasing the Coulombic efficiency during the Li plating/stripping process. Based on the above results, the significant advantages of CNF@Ni foam compared with planar $\mathrm{Cu}$ and Ni foam are evident and can be summarized below (as schemed in Figure 58): (i) the 3D conductive skeleton with porous structure can not only accommodate the volume change during the Li deposition process, thus increasing the structural stability; but also significantly improve the Li stripping efficiency, thus increasing the 
Coulombic efficiency; (ii) the CNFs on the surface can lower the local current density due to the increased effective surface area and enable uniform local current density distribution, therefore regulating a smooth Li deposition; (iii) the twisty CNFs as a heterogeneous phase during the Li deposition process, can act as nucleation sites to induce the uniform Li nucleation and growth along the CNFs, and also block the Li particles to form aggregates or even large dendrites.

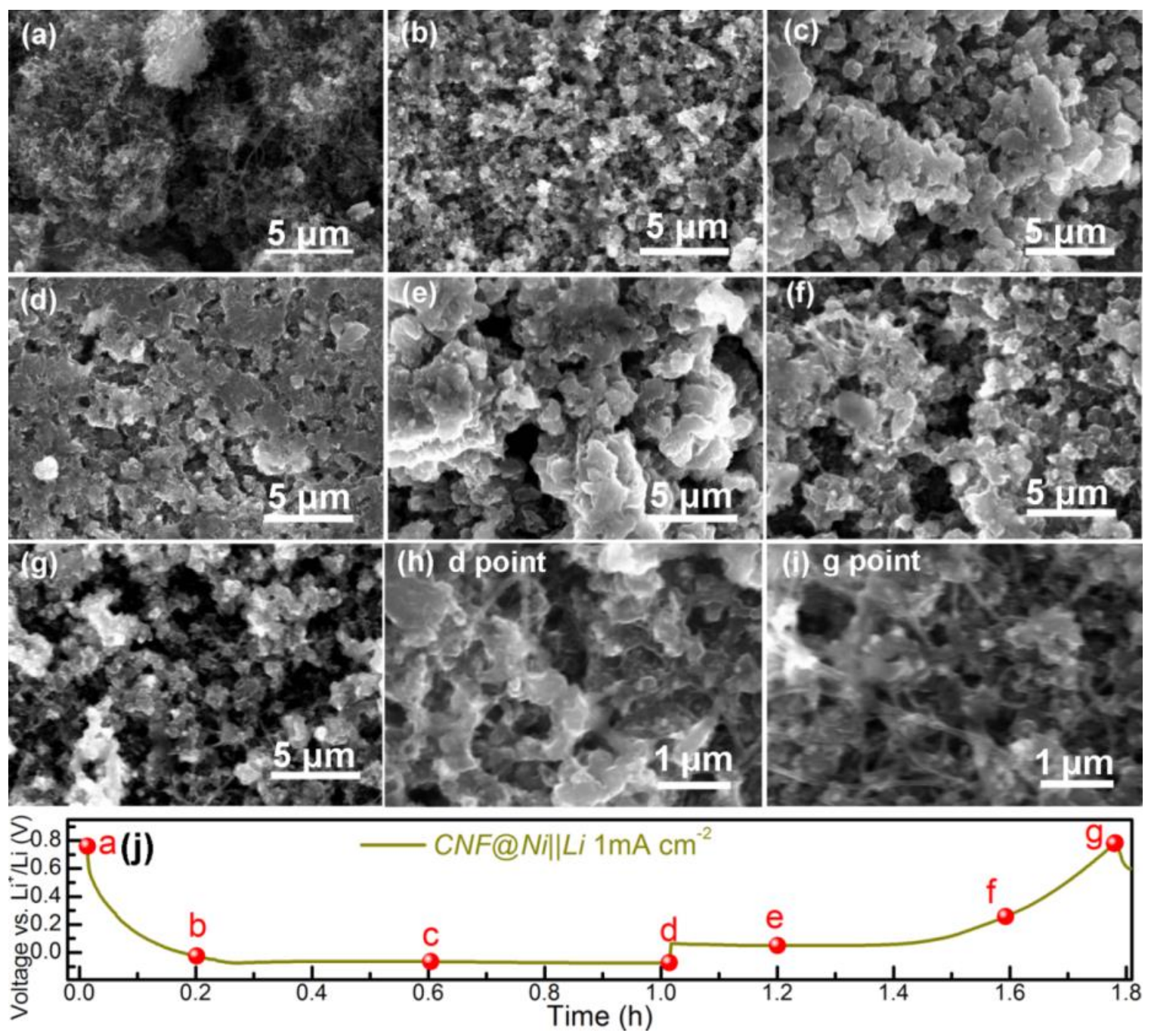

Figure 64 Morphological evolution of Li plating/stripping on CNF@Ni foam, including pristine CNF@Ni (a), and after plating at 0.2 (b), 0.6 (c), $1 \mathrm{mAh} \mathrm{cm}^{-2}$ (d) of Li, and after stripping at 0.2 (e), $0.6(\mathrm{f}), 1 \mathrm{mAh} \mathrm{cm}^{-2}$ (g) of Li from the Ni substrate. High magnification SEM images of $\mathrm{d}$ point (h) and g point (i). (j) Corresponding discharge/charge voltage profile of Ni||Li half-cell. 

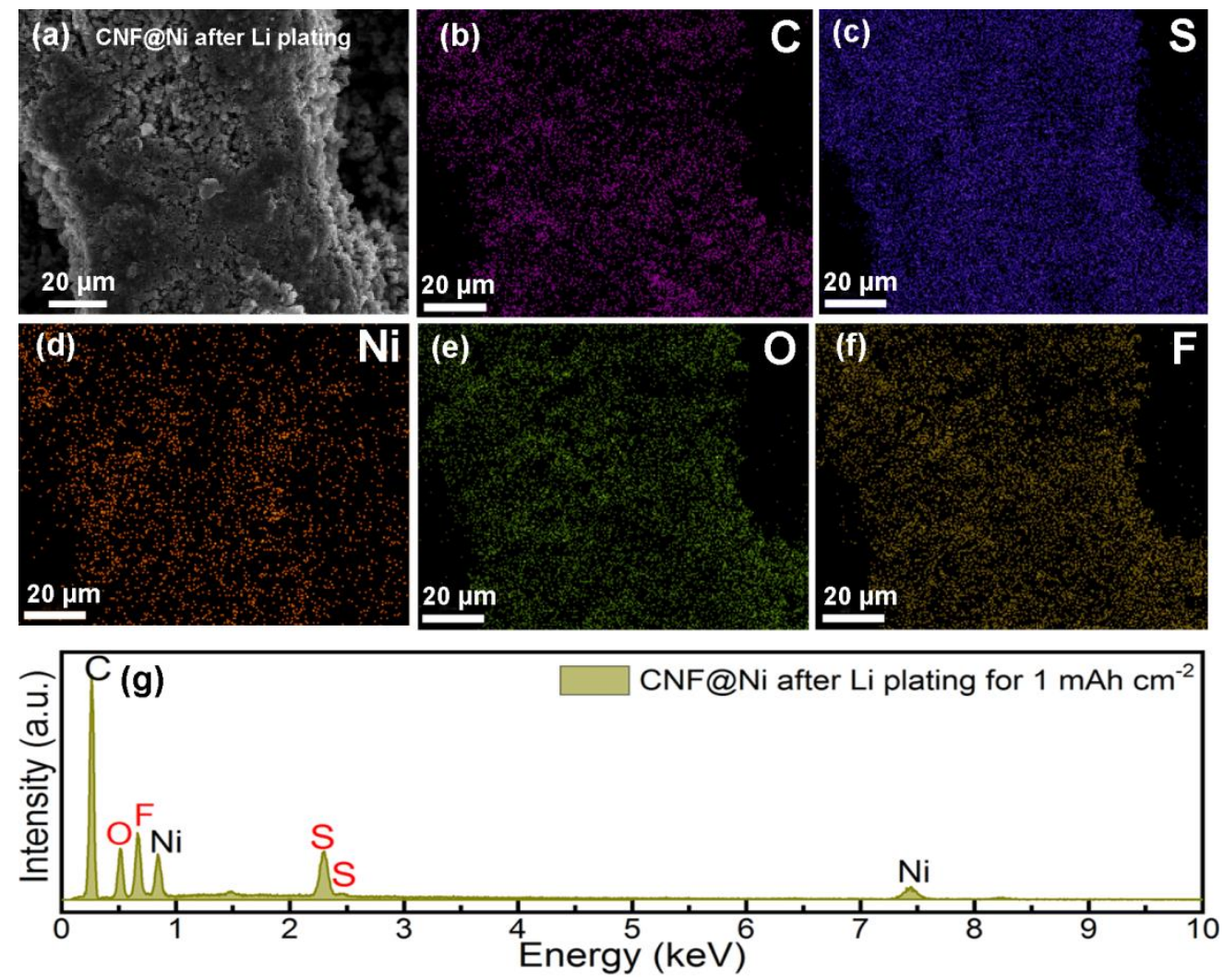

Figure 65 EDX mapping of CNF@Ni foam electrode after Li plating for $1 \mathrm{mAh} \mathrm{cm}^{-2}$ (a-f) and corresponding EDX spectrum (g).

To further evaluate the Li stripping/plating performance, the long-term deposition process of planar $\mathrm{Cu}, \mathrm{Ni}$ foam and CNF@Ni (as working electrodes) was investigated by using Li metal as the counter electrodes at current densities of $0.5,1$, and $2 \mathrm{~mA} \mathrm{~cm}{ }^{-2}$ with constant cycling capacity of $1 \mathrm{mAh} \mathrm{cm}^{-2}$. It can be clearly seen from Figure 66a-c that the uniform nucleation and deposition of Li on CNF@Ni foam can significantly improve the Coulombic efficiency (CE) of Li anode. Overall, the CNF@Ni foam shows a stable CE closed to 100\% during the 100 cycles. Even at the current density of $2 \mathrm{~mA} \mathrm{~cm}^{-2}$, the stripping and plating efficiency can be still stabled closed to $100 \%$. By comparison, the $\mathrm{Cu}$ foil and $\mathrm{Ni}$ foam show rapidly decreasing $\mathrm{CE}$ especially at higher current densities $\left(1 \mathrm{~mA} \mathrm{~cm}^{-2}\right.$ and $\left.2 \mathrm{~mA} \mathrm{~cm}^{-2}\right)$. The much higher CE of CNF@Ni foam than planar $\mathrm{Cu}$ and $\mathrm{Ni}$ foam unambiguously demonstrates that the uniform Li nucleation, 
deposition, and a reduced local current density are effective for regulating Li deposition. The corresponding charge-discharge profiles at the current density of $1 \mathrm{~mA} \mathrm{~cm}{ }^{-2}$ are presented in Figure 66d-f. However, the stripping capacity of $\mathrm{Cu}$ foil and $\mathrm{Ni}$ foam show fast decay. As to CNF@Ni foam, Li can still be completely stripped at $0.9 \mathrm{~V}$ with only slightly increased polarization after 100 cycles.

(a)
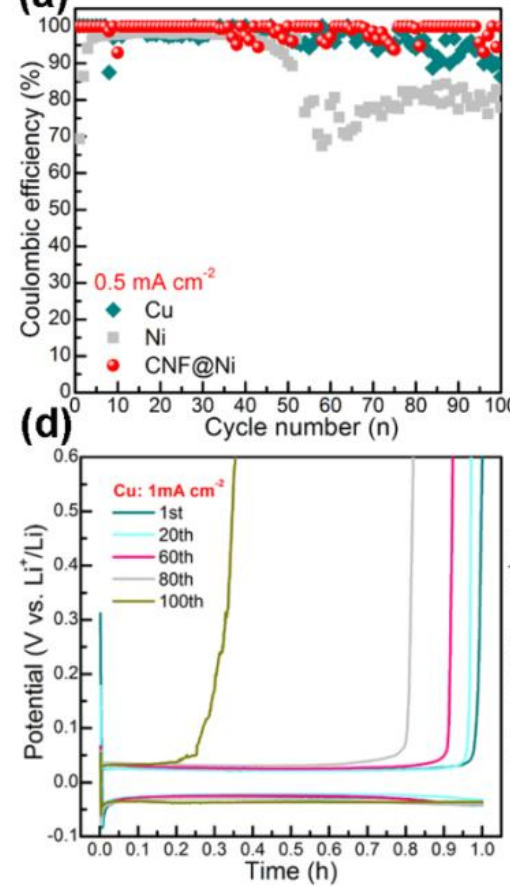

(g)

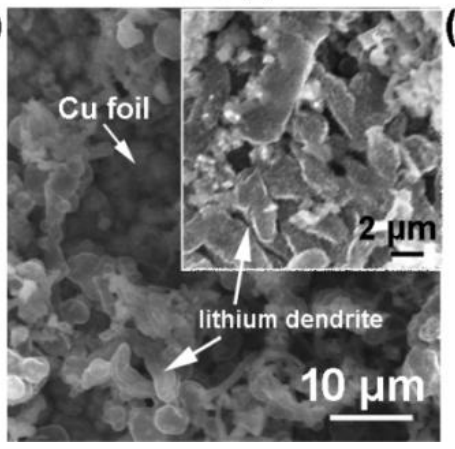

(b)

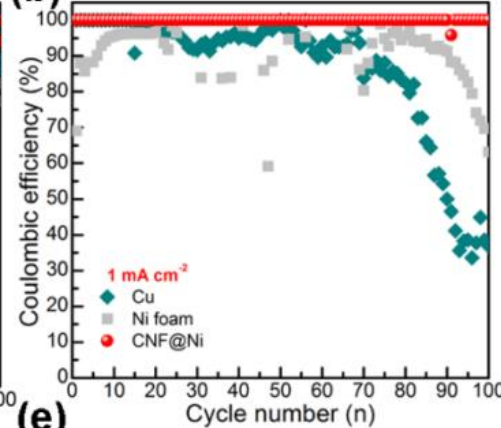

(e)

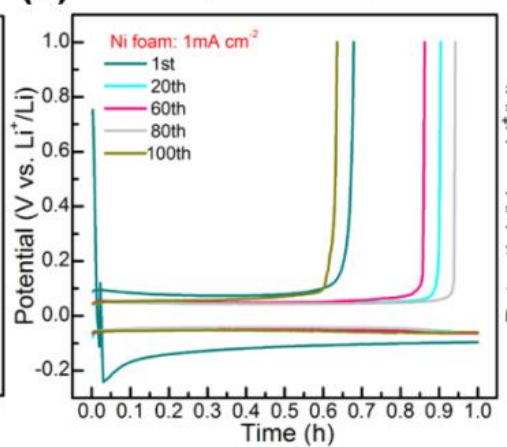

(h)

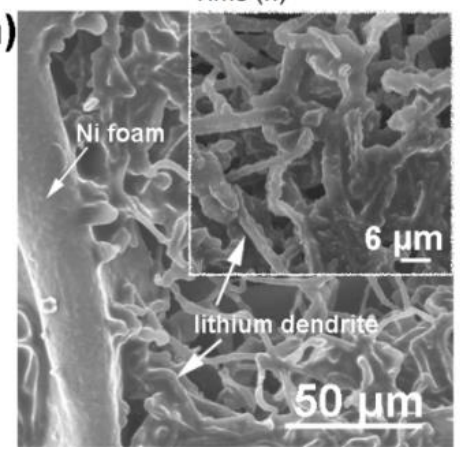

(c)
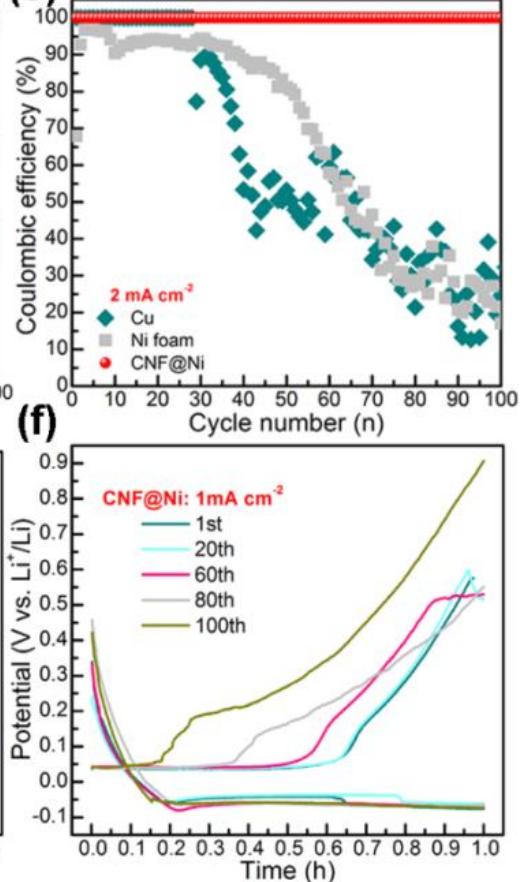

(i)

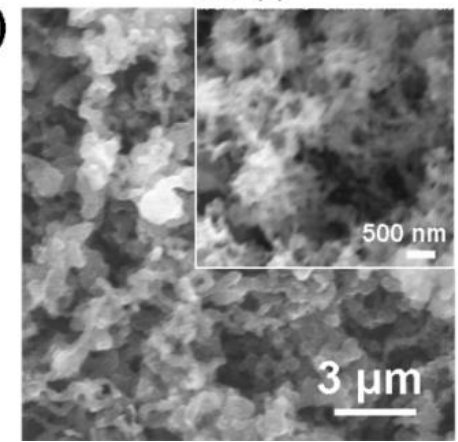

Figure $66 \mathrm{CE}$ of Li stripping from/plating on planar $\mathrm{Cu}$, Ni foam, and CNF@Ni foam at 0.5 (a), 1 (b), and $2 \mathrm{~mA} \mathrm{~cm}^{-2}$ with the constant areal capacity of $1 \mathrm{mAh} \mathrm{cm}^{-2}$ and the typical voltage profiles of the corresponding half cells (d-f). SEM images of $\mathrm{Cu}$ foil (g), Ni foam (h), and CNF@Ni foam (i) after 100 cycles stripping/plating at $1 \mathrm{~mA} \mathrm{~cm}^{-2}$ in half cells. 

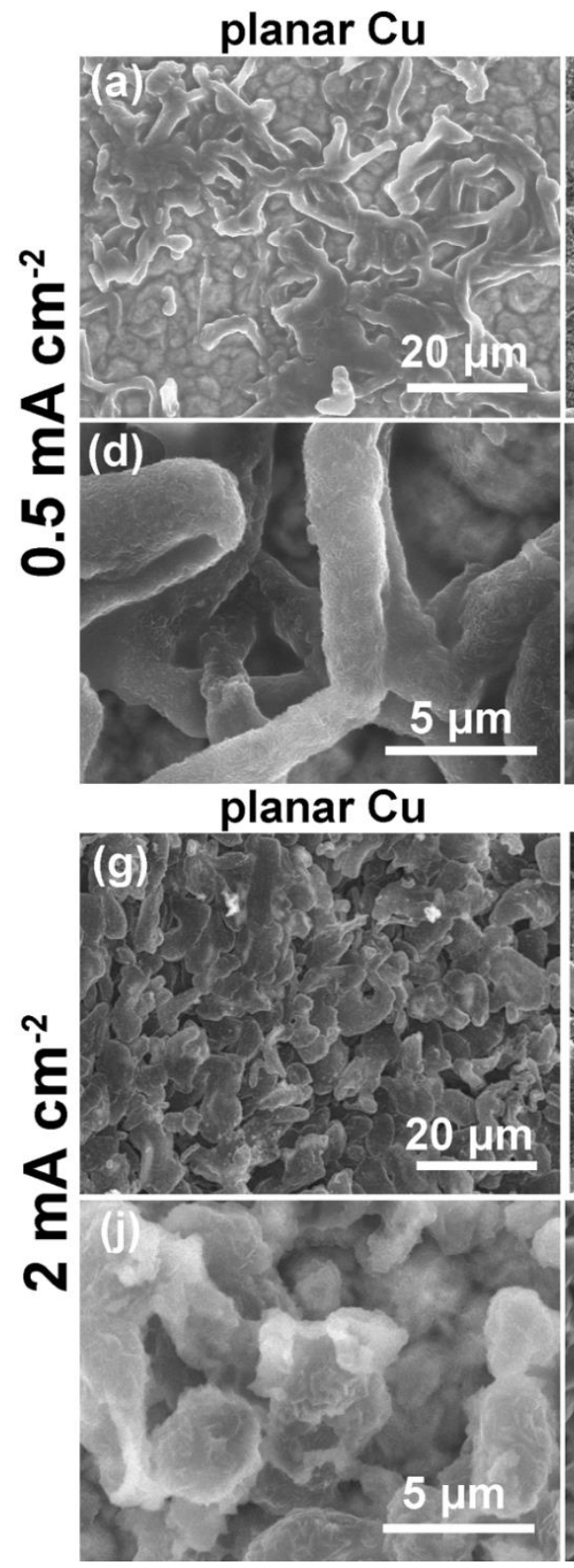

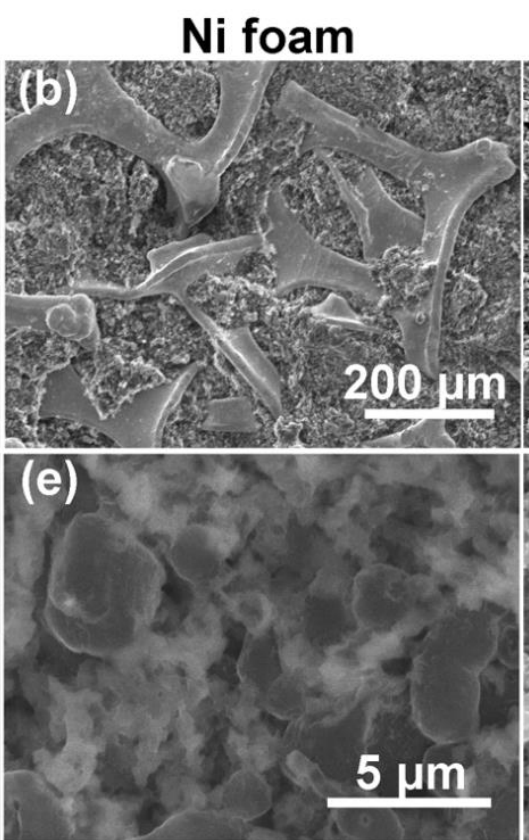

Ni foam
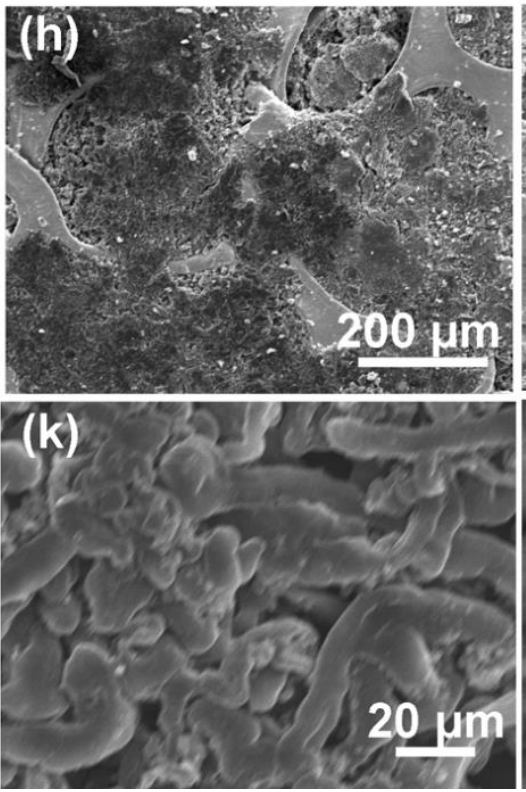

CNF@Ni foam

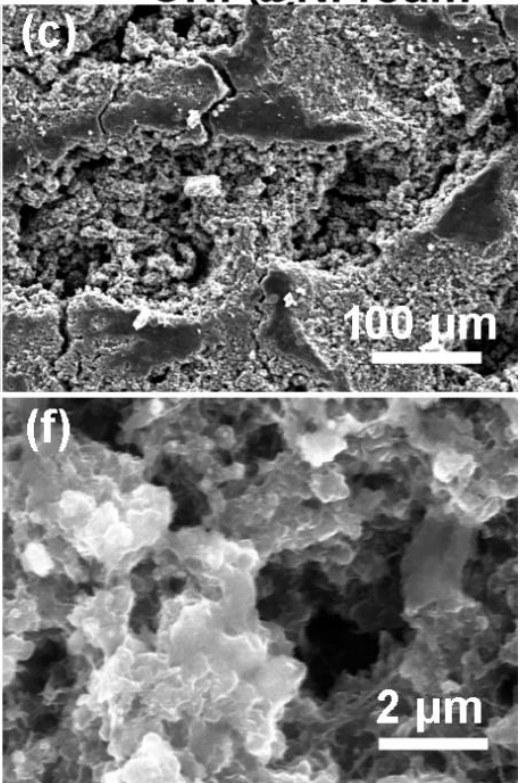

CNF@Ni foam

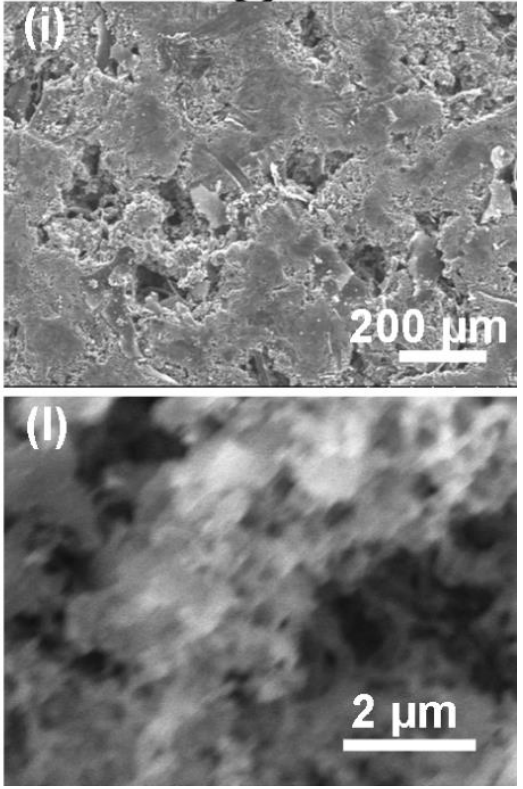

Figure 67 SEM images of planar $\mathrm{Cu}(\mathrm{a}, \mathrm{d}), \mathrm{Ni}$ foam (b, e) and CNF@Ni foam (c, f) after 100 cycles at $0.5 \mathrm{~mA} \mathrm{~cm}{ }^{-2}$, and planar $\mathrm{Cu}(\mathrm{g}, \mathrm{j}), \mathrm{Ni}$ foam (h, k) and $\mathrm{CNF} @ \mathrm{Ni}$ foam (i, l) after 100 cycles at $2 \mathrm{~mA} \mathrm{~cm}^{-2}$ in the corresponding half cells: $\mathrm{Cu}\|\mathrm{Li}, \mathrm{Ni}\| \mathrm{Li}$, and CNF@Ni $\mid \mathrm{Li}$.

The corresponding half cells were disassembled in the glove box and were transferred into the SEM chamber immediately in order to minimize the exposure of the samples into the air. Figure 
66 shows the surface SEM images of planar $\mathrm{Cu}$ foil, pure Ni foam, and CNF@Ni foam after 100 cycles stripping/plating. As expected, lots of Li dendrites with a diameter of $\sim 2 \mu \mathrm{m}$ covered on the surface of $\mathrm{Cu}$ foil. Some dendrites have already lost contact with the $\mathrm{Cu}$ substrate by exposing the $\mathrm{Cu}$ foil beneath (Figure 66g). As to the $\mathrm{Ni}$ foam, the Li dendrites with thick SEI can also be observed (Figure 66h). Notably, these dendrites are still connected and attached to the $\mathrm{Ni}$ foam to maintain the conductive network, thus showing a relatively higher Li stripping capacity and $\mathrm{CE}$ compared with planar $\mathrm{Cu}$. Obviously, the accelerating growth of $\mathrm{Li}$ dendrites can be achieved with the current density increasing from 0.5 to $2 \mathrm{~mA} \mathrm{~cm}^{-2}$ for planar $\mathrm{Cu}$ and pure $\mathrm{Ni}$ foam (Figure 67). However, the surface morphology of CNF@Ni seems not to change too much with increasing the current densities. The Li uniformly distributed in the CNFs matrix without any obvious large dendrites formed. The Li deposited along the CNFs, resulting in the slightly increased diameter of CNFs and amorphous-like surface morphology (Figure 66i) compared with the smooth and glassy surface of pristine CNFs (Figure 59). The large numbers of CNFs on Ni foam with uniform $\mathrm{Li}$ deposition and less "dead $\mathrm{Li}$ " also enable the much lower electrical resistance than the planar $\mathrm{Cu}$ and pure Ni foam. As confirmed by the Nyquist plots in Figure 68, the CNF@Ni electrodes after 100 cycles of stripping/plating process show much smaller interface impedances than $\mathrm{Cu}$ foil and pure $\mathrm{Ni}$ foam electrodes in all cases at the current densities of $0.5,1$, and $2 \mathrm{~mA} \mathrm{~cm}^{-2}$. 

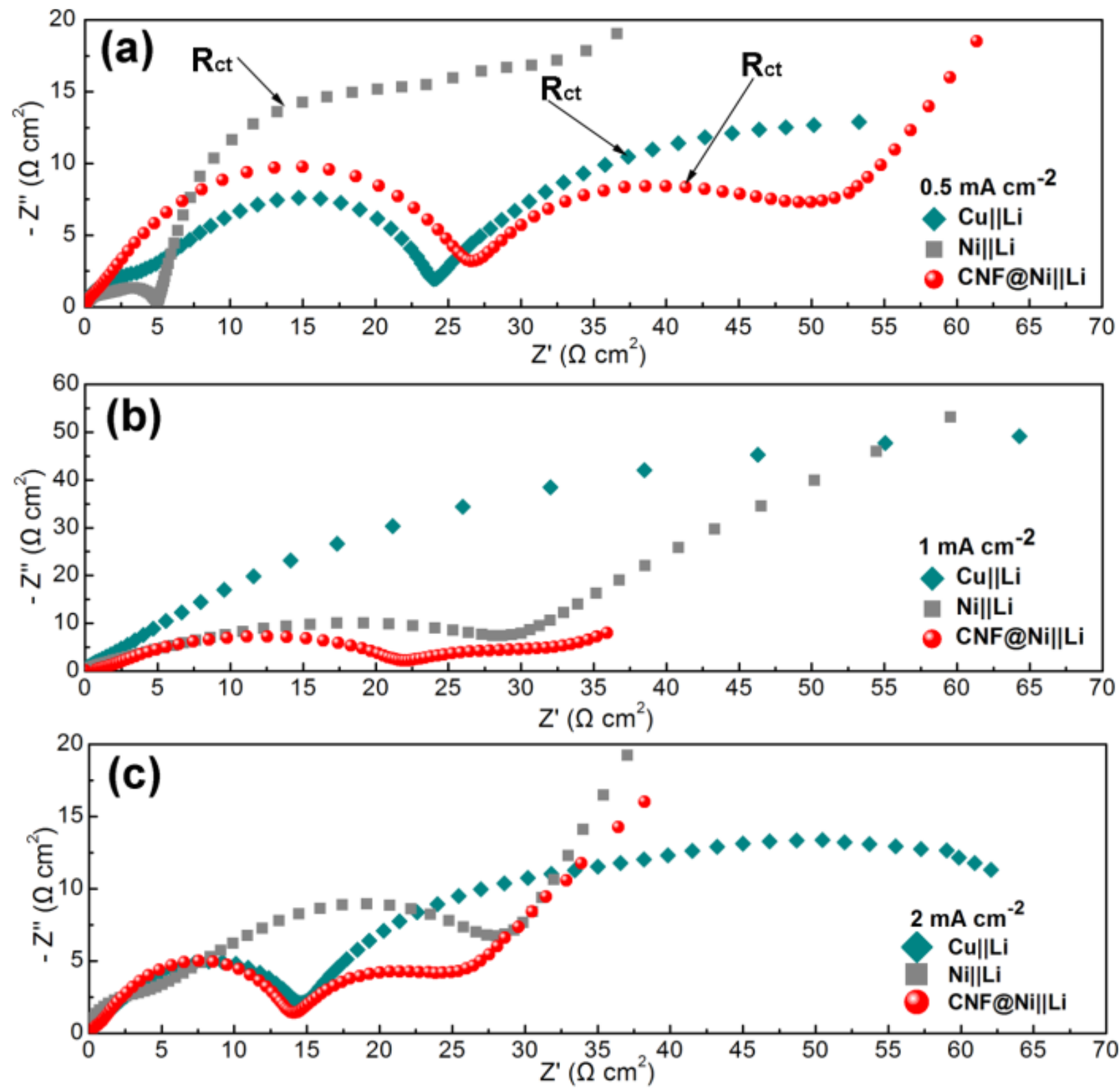

Figure 68 EIS Nyquist plots of half cells after 100 cycles at $0.5 \mathrm{~mA} \mathrm{~cm}^{-2}$ (a), $1 \mathrm{~mA} \mathrm{~cm}^{-2}$ (b), and $2 \mathrm{~mA} \mathrm{~cm}^{-2}(\mathrm{c})$.

\subsubsection{Electrochemical performance of symmetric cells}

Symmetric cells were assembled for testing to further verify that the CNF@Ni foam can enable stable Li stripping/plating behavior with the uniform Li deposition and high Coulombic efficiency. The planar $\mathrm{Cu}$, Ni foam, and $\mathrm{CNF} @ \mathrm{Ni}$ foam were firstly pre-plating with Li with the 
total $\mathrm{Li}$ plating capacity of $10 \mathrm{mAh} \mathrm{cm}^{-2}$. The Li predeposited electrodes $(\mathrm{Cu}-\mathrm{Li}$, Ni-Li, and CNF@Ni-Li) were extracted from the half cells to assemble symmetric cells. Long-term cycling stability of symmetric cells was conducted at various current densities. Figure 69a compares the stripping/plating behavior of the symmetric cells at $1 \mathrm{~mA} \mathrm{~cm}{ }^{-2}$ with constant cycling areal capacity of $1 \mathrm{mAh} \mathrm{cm}^{-2}$. The $\mathrm{Cu}-\mathrm{Li}$ electrode exhibits large polarization only after $10 \mathrm{~h}$, which then significantly increases during the following $80 \mathrm{~h}$. Ni-Li symmetric cells can compromise a longer lifespan with abrupt polarization appearing after 170 h. However, CNF@Ni-Li symmetric cell can cycle stably for $300 \mathrm{~h}$ with minimized polarization. Figure $69 \mathrm{~b}$ and c show the detail information of the voltage change profiles during the 0-10 and 100-110 h intervals, respectively. It can be clearly seen that CNF@Ni-Li electrodes exhibit smaller overpotential than both the Ni$\mathrm{Li}$ and $\mathrm{Cu}-\mathrm{Li}$ symmetric cells. The voltage hysteresis of $\mathrm{Cu}-\mathrm{Li}$ and $\mathrm{Ni}-\mathrm{Li}$ electrodes are really close due to the similarly "local surface". However, the voltage of the Cu-Li symmetric cell needs to reach $75 \mathrm{mV}$ to completely stripping and $-100 \mathrm{mV}$ to completely plating. Remarkably, the CNF@Ni-Li symmetric cell can keep a very stable voltage hysteresis, indicating the uniform electrode shape/volume due to the stable Li stripping/plating electrochemistry. The symmetric cells with $\mathrm{Cu}-\mathrm{Li}$ and $\mathrm{Ni}-\mathrm{Li}$ electrodes tested under various current densities of $0.5,2 \mathrm{~mA} \mathrm{~cm}{ }^{-2}$ for $100 \mathrm{~h}$ cycling are also compared, as shown in Figure 70. 

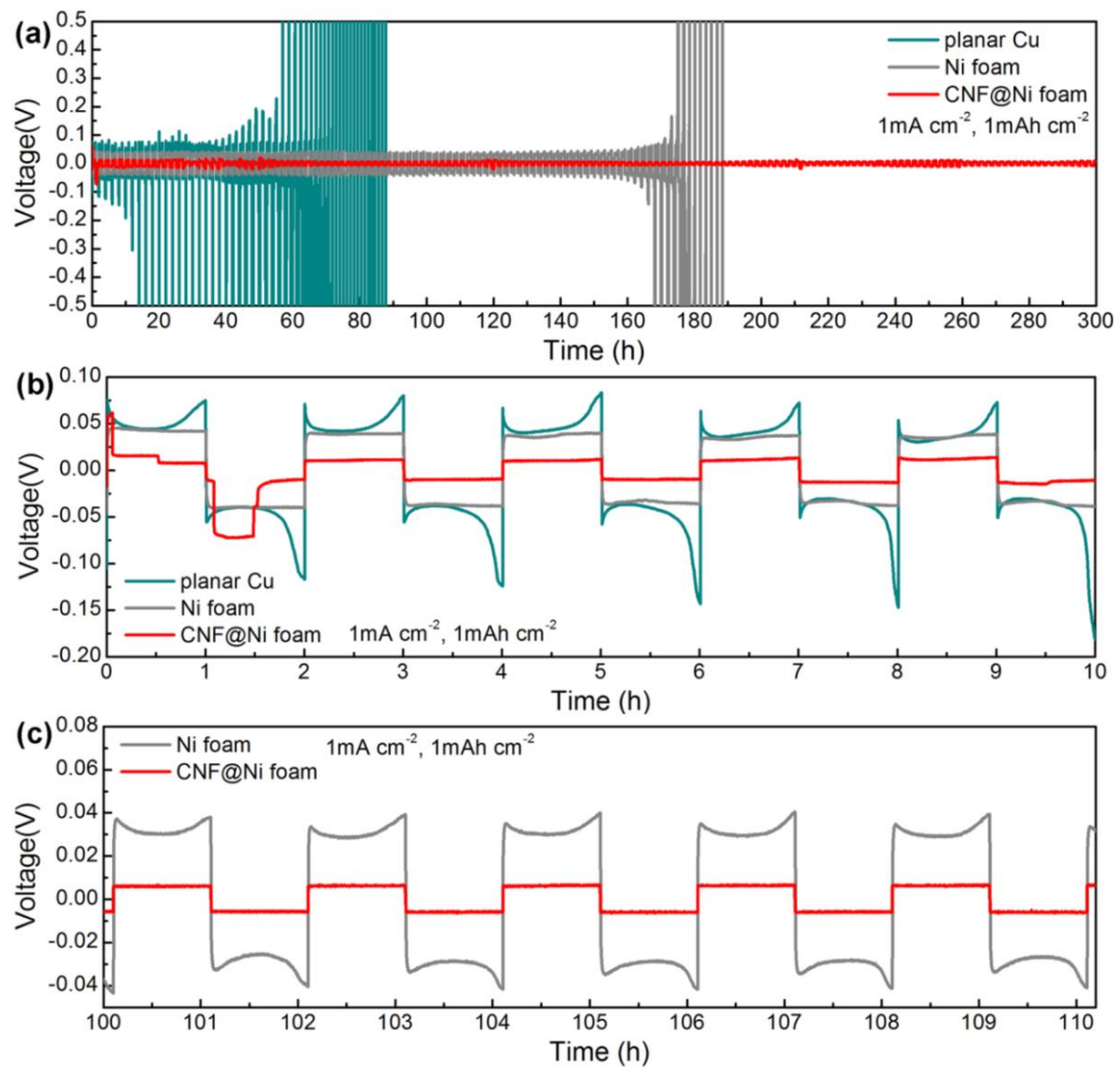

Figure 69 (a) Voltage-time profiles of Li stripping/plating with cycling capacity of $1 \mathrm{mAh} \mathrm{cm}$ at $1 \mathrm{~mA} \mathrm{~cm}{ }^{-2}$ in $\mathrm{Cu}-\mathrm{Li}, \mathrm{Ni}-\mathrm{Li}$, and CNF@Ni-Li symmetric cells. Magnified voltage profiles during $0-10 \mathrm{~h}(\mathrm{~b})$ and $100-110 \mathrm{~h}(\mathrm{c})$ periods. 

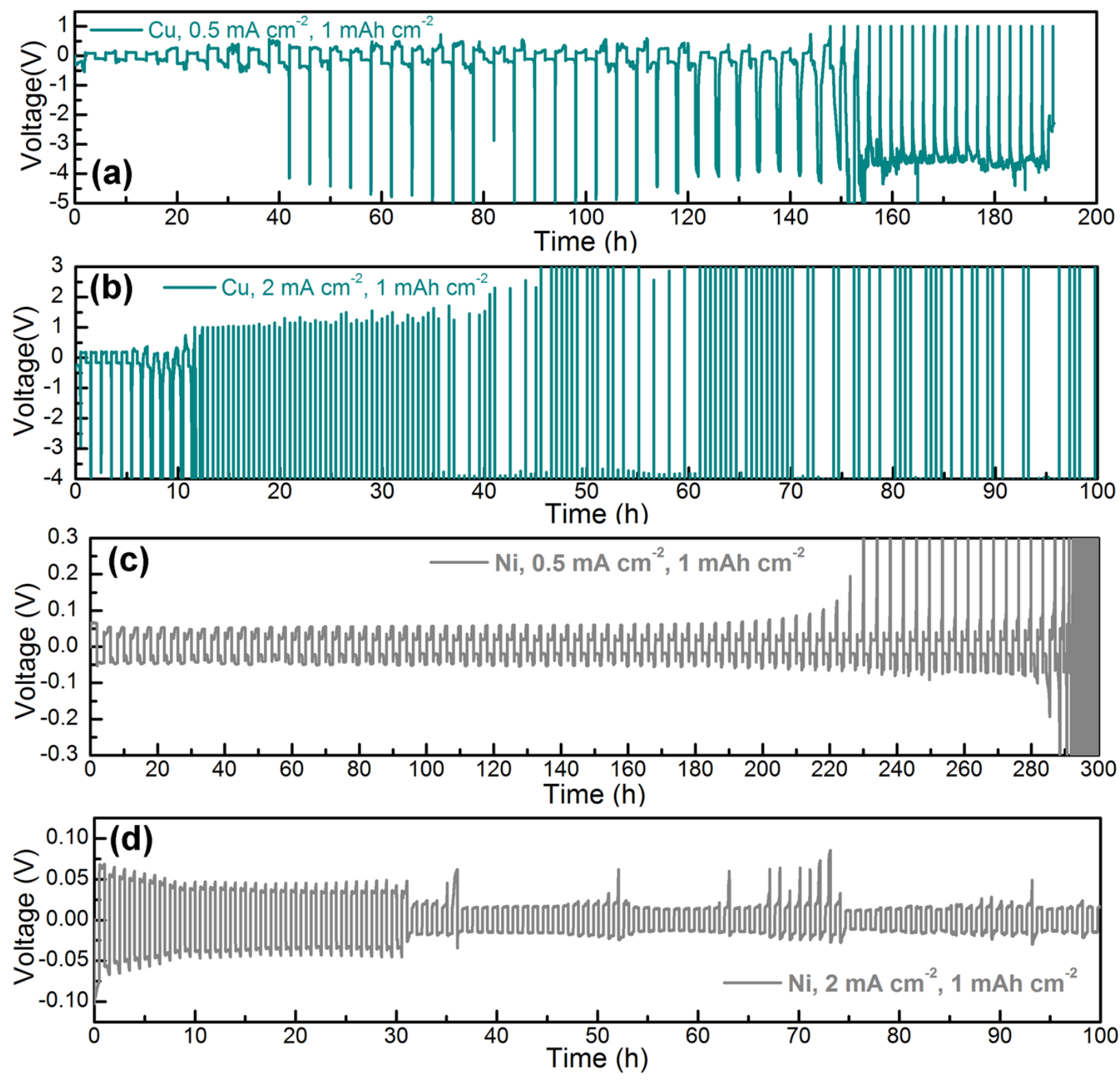

Figure 70 Symmetrical cells 0.5 and $2 \mathrm{~mA} \mathrm{~cm}^{-2}$ with a constant capacity of $1 \mathrm{mAh} \mathrm{cm}$ for 100 hours for $\mathrm{Cu}-\mathrm{Li}$ and $\mathrm{Ni}-\mathrm{Li}$ symmetric cells.

Figure 71a shows the voltage profiles of the Li plating/stripping process at various current densities for symmetric cells with CNF@Ni-Li electrodes. The CNF@Ni-Li symmetric cell shows a flat and stable voltage plateau with a smaller absolute overpotential value. This observation can be attributed to the fact that the electroactive surface area for CNF@Ni-Li 
electrodes is much larger under the same geometric dimensions. Consequently, the charge transfer resistance and polarization at the interface in the composite $\mathrm{Li}$ electrodes are reduced when the same amount of current is applied. The long-term symmetric cycling performance of the control and composite electrodes was conducted under various current densities of $0.5,2$, and $5 \mathrm{~mA} \mathrm{~cm}{ }^{-2}$ with a constant areal capacity of $1 \mathrm{mAh} \mathrm{cm}^{-2}$, as shown in Figure 70 and Figure 71bd. Symmetric cells using $\mathrm{Cu}-\mathrm{Li}$ and Ni-Li electrodes exhibit gradually increasing polarization until an abrupt voltage drop, thus implying an uncontrolled Li dendrite growth, followed by internal short-circuiting (Figure 70). This change mainly originates from the fluctuating electrode shape/volume during a hostless deposition/extraction. However, CNF@Ni-Li symmetric cells can cycle stably for nearly $1000 \mathrm{~h}$ under high current densities of $0.5 \mathrm{~mA} \mathrm{~cm}{ }^{-2}$. When current densities increased to $2 \mathrm{~mA} \mathrm{~cm}{ }^{-2}$ (Figure 71c) and $5 \mathrm{~mA} \mathrm{~cm}^{-2}$ (Figure 71d), the CNF@Ni-Li symmetric cells still exhibit low voltage hysteresis (Figure 72) without any dendrite-induced failure during 300 hours, showing excellent cycle stability. These results indicate that ramified Li dendrite formation is lessened because of the reduced local current density due to the significantly increased effective specific surface area and the presence of CNFs and metal nanoparticles as heterogeneous phases to enable a uniform "Li nucleation" and "electric field" for the following Li deposition and growth process. 


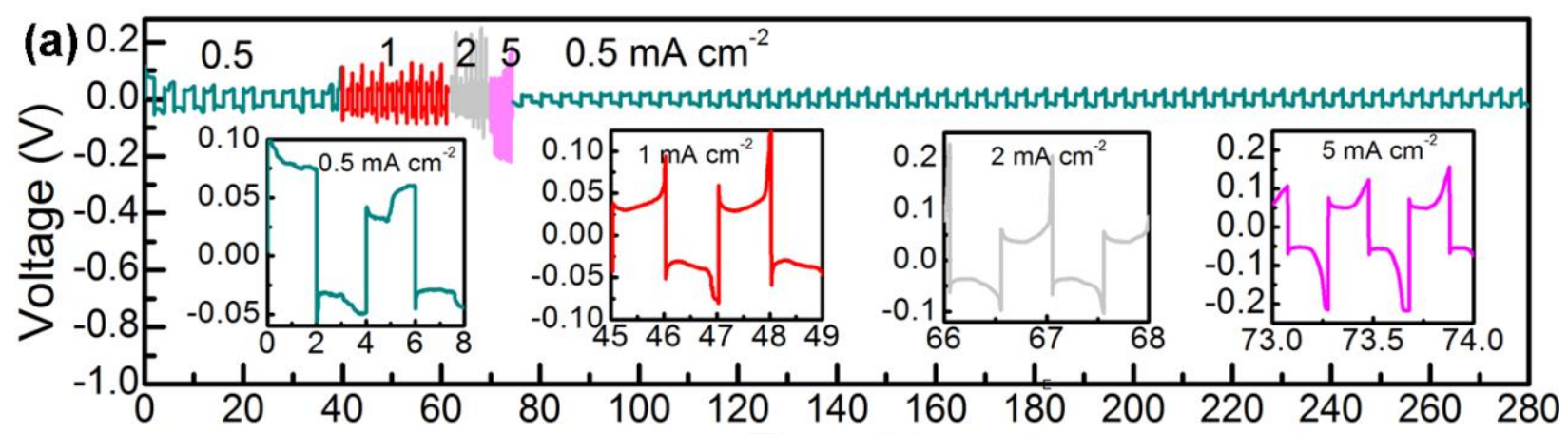

(b)

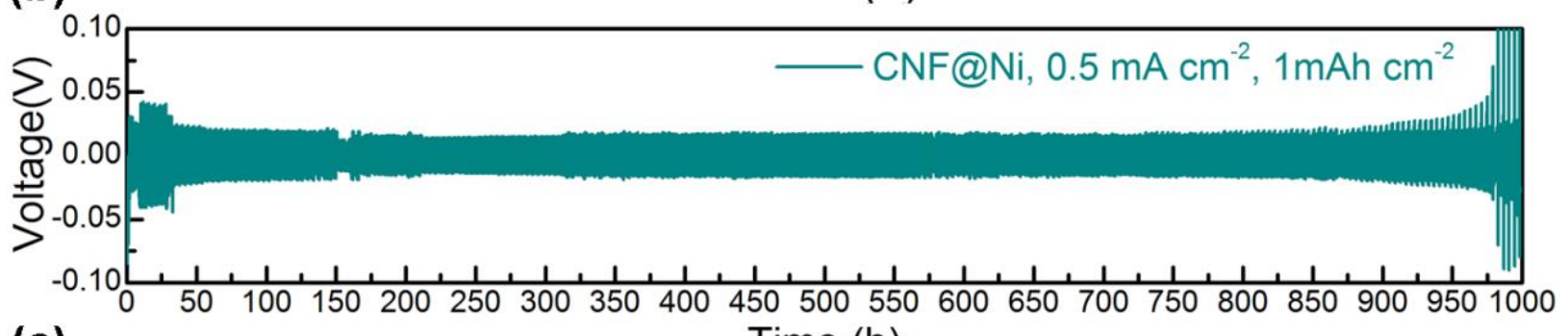

(c)
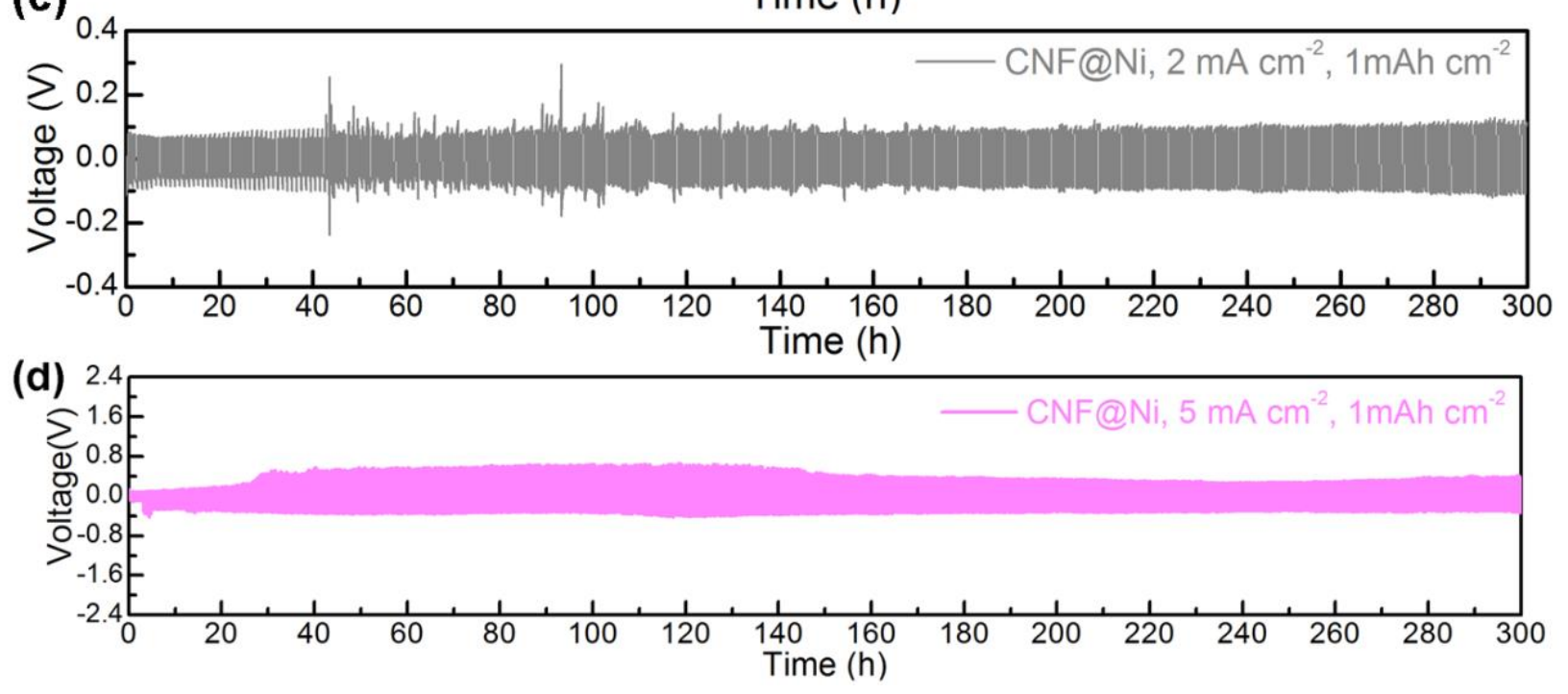

Figure 71 Electrochemical performances of CNF@Ni-Li symmetric cells. (a) Voltage profiles during Li plating/stripping processes under various current rates ranging from 0.5 to $5 \mathrm{~mA} \mathrm{~cm}^{-2}$. (b to c) Long-term cycling of CNF@Ni-Li symmetric cells with current densities of 0.5 , 2, and 5 $\mathrm{mA} \mathrm{cm} \mathrm{cm}^{-2}$ with a deposition/stripping capacity of $1 \mathrm{mAh} \mathrm{cm}^{-2}$. 

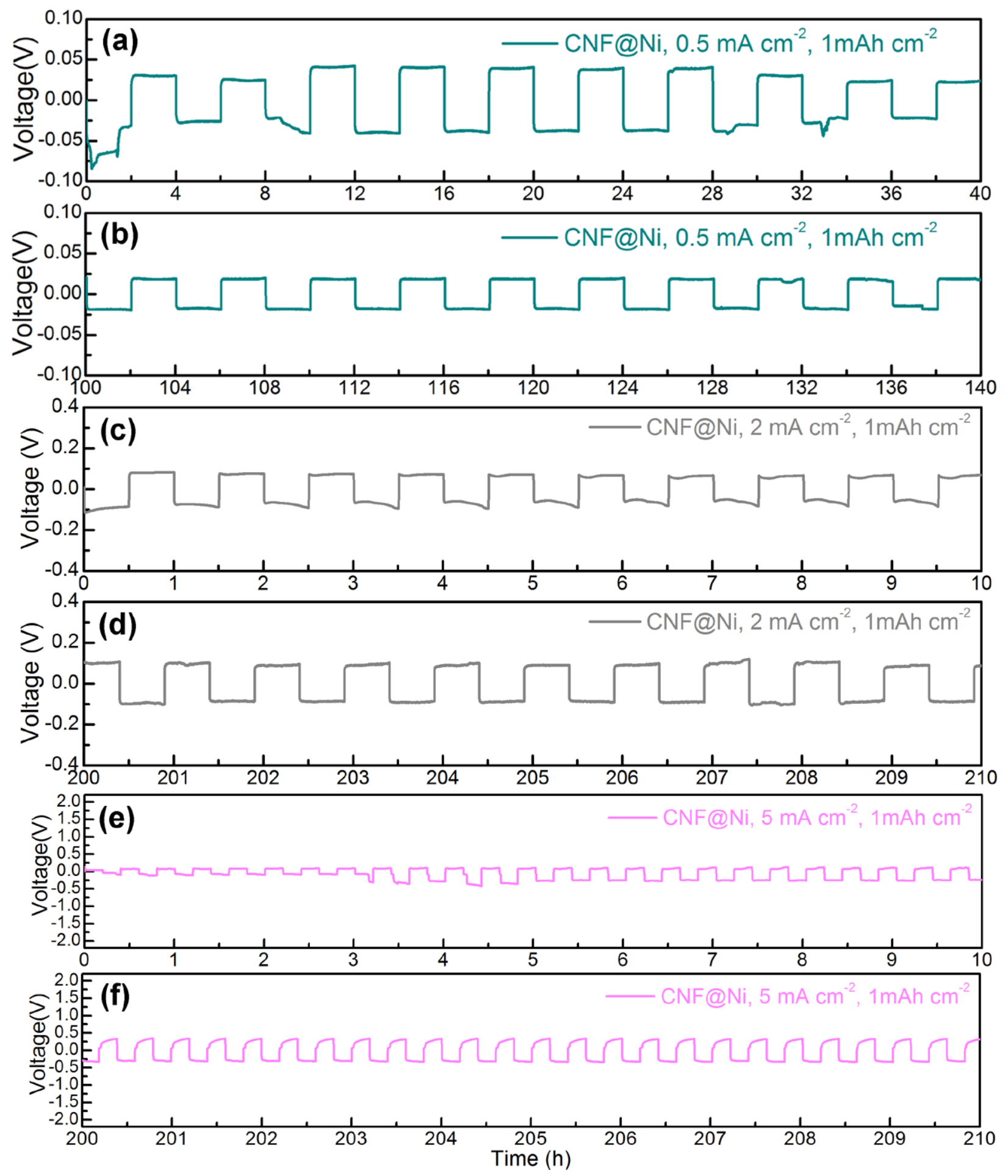

Figure 72 Magnified Li plating/stripping profiles of CNF@Ni-Li symmetric cells during different cycle periods. 
To further confirm the advantages of CNF@Ni-Li electrodes, the morphology of planar Cu-Li, Ni-Li foam, and CNF@Ni-Li electrodes were extracted from the corresponding symmetric cells and examined both from the top and side views after cycling measurements. Figure 73a-c show the surface morphology of the $\mathrm{Cu}-\mathrm{Li}, \mathrm{Ni}-\mathrm{Li}$, and CNF@Ni-Li electrodes after cycling at $1 \mathrm{~mA}$ $\mathrm{cm}^{-2}$ for 100 cycles, respectively. Obviously, the planar $\mathrm{Cu}-\mathrm{Li}$ electrode shows lots of dendrites covering on the surface with diameters of $\sim 1 \mu \mathrm{m}$. Similarly, a large amount of Li dendrites can be found on/in the surface and pores of the Ni-Li electrode. However, the CNF@Ni-Li electrode shows much clean surface morphology without large dendrites formed. A much more obtuseshaped Li deposition along the carbon nanofibers instead of a sharp Li dendrite can be observed. The cross-section images further illustrate the morphology and distribution of deposited Li. The thickness of $\mathrm{Li}$ dendrite on the planar $\mathrm{Cu}$ and pure $\mathrm{Ni}$ foam is around 10 and $50 \mu \mathrm{m}$, respectively. However, the Li deposited in the carbon matrix of $\mathrm{CNF} @ \mathrm{Ni}$ foam without accumulating to form large bulk and dense "lithium layer". The majority of pores in the foam can still be maintained as well as the spatial voids between fibers (Figure 73c). It should be also noticed that the substrates of both planar $\mathrm{Cu}$ (Figure 73a) and Ni foam (Figure 73b) can be observed even after 100 cycles. Due to the large nucleation overpotential of planar $\mathrm{Cu}$ and pure $\mathrm{Ni}$ foam, the nucleation process during the Li deposition is not uniform. The uneven distributed Li seed crystals on the surface will induce the dendritic Li growth in the subsequent cycling, resulting in the exposed substrates on the surface. However, the CNFs on the Ni foam as a heterogeneous phase provides a lot of active sites for $\mathrm{Li}$ deposition, facilitating a uniform Li nucleation as well as the following $\mathrm{Li}$ growth by reducing the nucleation overpotential. Li preferably deposited in the carbon matrix and maintaining the open pores of $\mathrm{Ni}$ foam skeleton, which can further promote the locally uniform $\mathrm{Li}^{+}$flux and accommodate the large volume change. 
The stable Li stripping and plating electrochemistry can be corroborated by the lower voltage hysteresis and interface impedances (EIS). As summarized in Figure 73g, the CNF@Ni-Li symmetric cells show much lower and more stable voltage hysteresis when cycling at $0.5,1$, and $2 \mathrm{~mA} \mathrm{~cm}^{-2}$. However, $\mathrm{Cu}-\mathrm{Li}$ and Ni-Li symmetric cells show significant polarization and even short circuits after 40 cycles. Correspondingly, EIS curves of symmetric cells after 100 cycles in Figure 73h reveal the significantly reduced polarization of CNF@Ni-Li electrodes. The SEI interfacial resistance and the $\mathrm{Li}$ surface charge transfer impedance are estimated from the semicircle at a high-frequency and mid-high frequency range, respectively. In all cases, the interfacial resistances of CNF@Ni-Li symmetric cells are much lower than those of $\mathrm{Cu}-\mathrm{Li}$ and $\mathrm{Ni}-\mathrm{Li}$ electrodes. The uneven deposited $\mathrm{Li}$ on planar $\mathrm{Cu}$ and pure $\mathrm{Ni}$ foam with large formation of "dead Li" impede the reaction kinetics. However, the regulated Li deposition and extraction in CNF@Ni foam with 3D electronic conductive network structure keeps the stable shape and volume during the cycling, resulting in the constantly low interfacial resistances when tested at all current densities. These results further demonstrate the excellent capability of CNF@Ni foam to enable the uniform Li distribution with stable morphology of electrode and much better $\mathrm{Li}$ stripping and plating kinetics. Such stable Li stripping/plating electrochemistry of the CNF@Ni$\mathrm{Li}$ electrode makes it stand out among recently reported Li anodes, as compared in Table 8. 

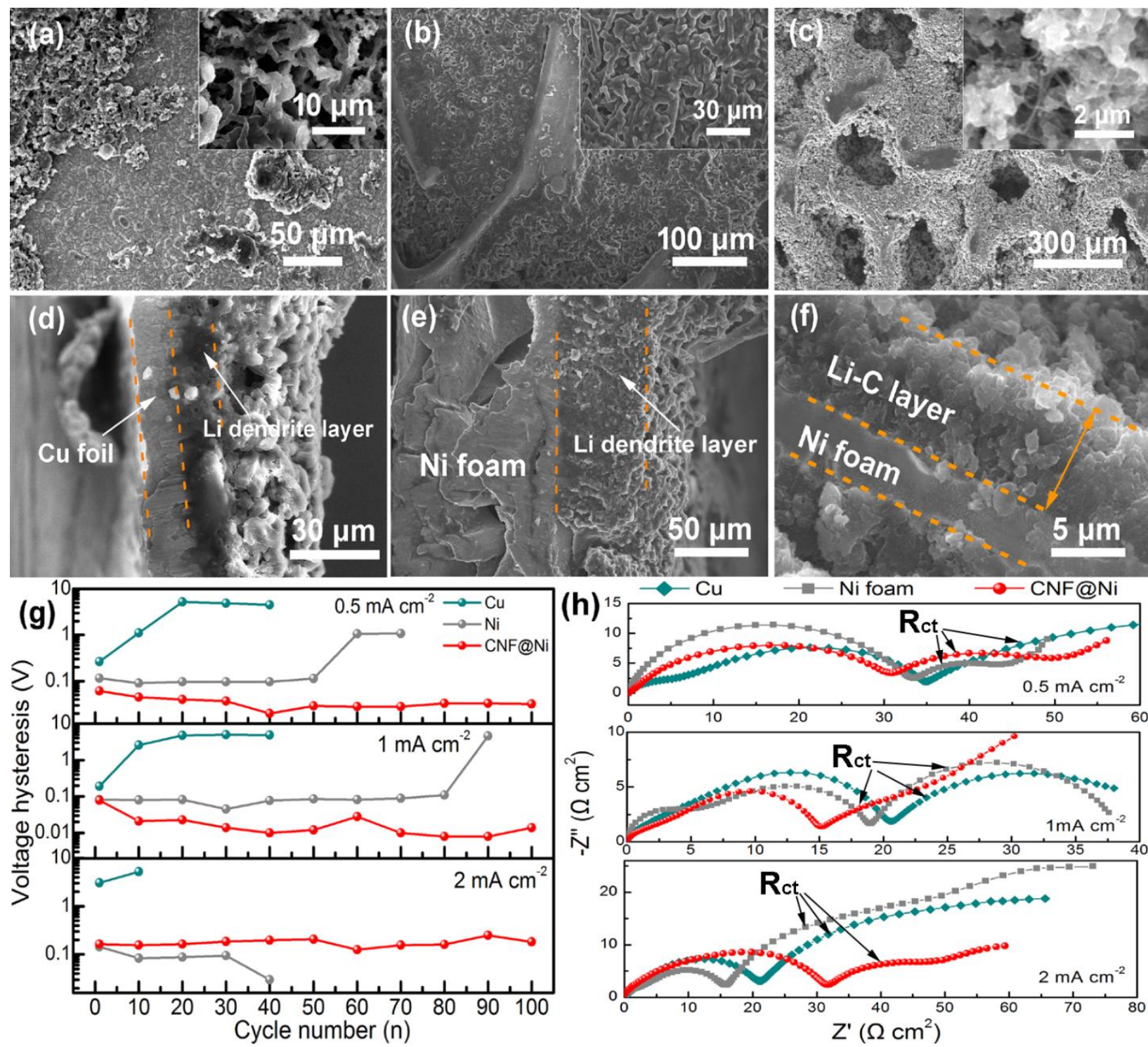

Figure 73 Surface (a-c) and cross-sectional (d-f) SEM images of Cu-Li (a, d), Ni-Li (b, e), and CNF@Ni (c. f) electrodes after 100 cycles in the corresponding symmetric cells. (g) Summary of voltage hysteresis for the $\mathrm{Cu}-\mathrm{Li}, \mathrm{Ni}-\mathrm{Li}$, and $\mathrm{CNF} @ \mathrm{Ni}-\mathrm{Li}$ symmetric cells at various current densities. (h) EIS Nyquist curves of symmetric cells after 100 cycles at various current densities of $0.5,1$, and $2 \mathrm{~mA} \mathrm{~cm}^{-2}$. 
Table 8 The performance comparison of the CNF@Ni-Li electrode with reported Li anodes.

\begin{tabular}{|c|c|c|c|c|c|}
\hline $\begin{array}{l}\text { Cathodic } \\
\text { materials }\end{array}$ & $\begin{array}{c}\text { Current } \\
\left.(\mathrm{mA} \mathrm{cm})^{-2}\right)\end{array}$ & $\begin{array}{c}\text { Capacity } \\
\left(\mathrm{mAh} \mathbf{c m}^{-2}\right)\end{array}$ & Cycle life & $\begin{array}{c}\text { Volatge } \\
\text { hysteresis } \\
(\mathrm{mV})\end{array}$ & Reference \\
\hline \multirow{4}{*}{ CNF@Ni-Li } & 0.5 & 1 & $\sim 1000 \mathrm{~h}$ & 50 & \multirow{4}{*}{ This work } \\
\hline & 2 & $\mathbf{1}$ & $300 \mathrm{~h}$ & 200 & \\
\hline & 5 & 1 & $300 \mathrm{~h}$ & 350 & \\
\hline & 1 & 5 & $1000 \mathrm{~h}$ & 150 & \\
\hline Li-CMN & 1 & 1 & $600 \mathrm{~h}$ & 80 & {$[133]$} \\
\hline 3D Li & 0.2 & 0.1 & $700 \mathrm{~h}$ & 200 & {$[206]$} \\
\hline \multirow{3}{*}{ Co-CS-Li } & 1 & 1 & $800 \mathrm{~h}$ & 50 & \multirow{3}{*}{ [207] } \\
\hline & 3 & 1 & $180 \mathrm{~h}$ & 100 & \\
\hline & 5 & 1 & $70 \mathrm{~h}$ & 140 & \\
\hline \multirow{2}{*}{$\mathrm{g}-\mathrm{C}_{3} \mathrm{~N}_{4} @ \mathbf{N i}$} & 1 & 1 & $900 \mathrm{~h}$ & 20 & \multirow[t]{2}{*}{ [203] } \\
\hline & 2 & 1 & $500 \mathrm{~h}$ & 40 & \\
\hline 3D Cu & 0.2 & 0.5 & $600 \mathrm{~h}$ & $<50$ & {$[127]$} \\
\hline \multirow{3}{*}{$\mathbf{L i}-\mathbf{N i}$} & 1 & 1 & $200 \mathrm{~h}$ & 100 & \multirow{3}{*}{ [208] } \\
\hline & 3 & 1 & $70 \mathrm{~h}$ & 250 & \\
\hline & 5 & 1 & $40 \mathrm{~h}$ & 400 & \\
\hline \multirow{3}{*}{ GF-LiF-Li } & 1 & 1 & $240 \mathrm{~h}$ & 70 & \multirow{3}{*}{ [209] } \\
\hline & 5 & 1 & $55 \mathrm{~h}$ & 500 & \\
\hline & 10 & 1 & $30 \mathrm{~h}$ & 800 & \\
\hline$\alpha-\mathrm{Si}_{3} \mathrm{~N}_{4}-\mathbf{L i}$ & 1 & 1 & $3000 \mathrm{~h}$ & 40 & {$[210]$} \\
\hline \multirow{2}{*}{$\mathrm{Cu}-\mathrm{CuO}-\mathrm{Ni}-10$} & 1 & 1 & $<600 \mathrm{~h}$ & $\sim 30$ & \multirow[t]{2}{*}{ [204] } \\
\hline & 3 & 0.5 & $\sim 25 \mathrm{~h}$ & $\sim 130$ & \\
\hline Graphene flake & 2 & 0.1 & $\sim 70 \mathrm{~h}$ & $\sim 150$ & {$[211]$} \\
\hline \multirow{2}{*}{$\mathrm{Li} / \mathrm{CF}$} & 0.25 & 0.5 & $750 \mathrm{~h}$ & $\sim 50$ & \multirow[t]{2}{*}{ [212] } \\
\hline & 0.5 & 0.5 & $560 \mathrm{~h}$ & $\sim 80$ & \\
\hline 3D Copper & 1 & 1 & $400 \mathrm{~h}$ & $\sim 40$ & [213] \\
\hline $\mathbf{L i}_{2} \mathbf{S} @ \mathbf{L i}$ & 2 & 5 & $750 \mathrm{~h}$ & $\sim 220$ & {$[214]$} \\
\hline \multirow{3}{*}{ LiF-rich Li } & 1 & 1 & $550 \mathrm{~h}$ & 100 & \multirow{3}{*}{ [215] } \\
\hline & 2 & 1 & $400 \mathrm{~h}$ & 60 & \\
\hline & 5 & 1 & $\sim 400 \mathrm{~h}$ & 300 & \\
\hline PPS@Li & 1 & 2 & 1000 & $\sim 300$ & [216] \\
\hline \multirow{2}{*}{ PAA@Li } & 1 & 1 & $800 \mathrm{~h}$ & $\sim 50$ & \multirow[t]{2}{*}{ [217] } \\
\hline & 5 & 5 & $350 \mathrm{~h}$ & $\sim 200$ & \\
\hline $\mathrm{TiO}_{2} / \mathrm{ROLi}-\mathrm{Li}$ & 1 & 1 & $600 \mathrm{~h}$ & 100 & {$[218]$} \\
\hline LN-1 & 0.5 & 1 & $<500 \mathrm{~h}$ & 200 & \multirow{4}{*}{ [219] } \\
\hline LN-2 & 0.5 & 1 & $500 \mathrm{~h}$ & 150 & \\
\hline LN-2 & 1 & 2 & $200 \mathrm{~h}$ & $\sim 300$ & \\
\hline LN-3 & 0.5 & 1 & $\sim 500 \mathrm{~h}$ & 300 & \\
\hline Li-Cu-Li arrays & 1 & 5 & $800 \mathrm{~h}$ & $\sim 250$ & {$[220]$} \\
\hline
\end{tabular}




\subsubsection{Mechanism for stable Li stripping/plating electrochemistry}

Based on the results above, it is evident that CNF@Ni foam electrodes can afford much more uniform Li stripping and plating morphology due to the lower Li nucleation overpotential, more homogeneously distributed current density and $\mathrm{Li}^{+}$concentration. As schemed in Figure 74a-b, the small Li dendrite previously deposited on the $\mathrm{Cu}$ and $\mathrm{Ni}$ foam can function as a charge center due to the accumulation of charges at the sharp ends in the electric field. During the subsequent process, $\mathrm{Li}^{+}$will preferably deposit on these sharp ends and continuously grow to form dendritic Li. In contrast, on the 3D CNF@Ni foam, the nanoscale carbon fibers can provide numerous protuberant tips as the charge centers and nucleation sites. The electric potential is roughly uniform and the charges are fairly homogeneously dispersed along the CNFs. Therefore, $\mathrm{Li}$ is expected to nucleate and grow along the carbon nanofibers, and eventually form nanosized lumps (Figure 64g), fill into the pores of $\mathrm{Ni}$ foam skeleton (Figure 67) with relatively even $\mathrm{Li}$ deposition morphology. To further confirm this hypothesis, the distribution of the electric potential and current density on the planar $\mathrm{Cu}$ (Figure 74d-f) and CNF@Ni (Figure 74h-j) electrode were simulated using the finite volume method. The electrical potential is represented by the intensity by the color, and the current density distributions are shown by the arrows. A simplified 2D electrodeposition model was established to compare the electric potential and current density distribution during cycling. The corresponding parameters in the models based on planar $\mathrm{Cu}$ and CNF@Ni foam electrodes are illustrated in Figure 74d and g, respectively. The results were obtained by solving the following partial differential equations with the numerical scheme:

$$
\nabla \cdot(-\sigma \nabla \varphi)=0
$$


where $\sigma$ is the electrical conductivity of the conducting materials, and $\varphi$ is the electrical potential. With the electrical potential $\varphi$ obtained from the numerical simulation, the current density $\vec{\imath}$ can be calculated based on Ohm's law:

$$
\vec{\imath}=\sigma \nabla \varphi
$$

In the present study, the finite volume method was utilized to solve the aforementioned partial differential equations. The whole computational zone was divided into lots of small finite volumes by the uniform Cartesian mesh. For all the surfaces of each small volume, the fluxes entering/leaving the volumes as well as the source/sink terms (if any) are calculated to finally form the governing equations for each volume. The boundary conditions are the cathodic potentials are set based on the true voltage hysteresis at different current densities, while the anodic potentials are constantly set as $0 \mathrm{~V}$. As shown in Figure $74 \mathrm{e}$, the electrical potential distribution on the planar $\mathrm{Cu}$ surface with trapezoidal Li seeds is inhomogeneous, as indicated by the wavy green region. The corresponding current density (Figure 74f) distribution reveals that a much higher charge region in the close vicinity of the isolated Li nuclei sites than other areas (labeled in red). The obvious intensity gradient is capable of driving more $\mathrm{Li}^{+}$flux to adsorb onto individual nucleation centers, causing continuously uneven Li deposition. During cycling, $\mathrm{Li}^{+}$ prefers to continuously deposit on these protuberances sites, and consequently resulting in $\mathrm{Li}$ dendrite growth. In contrast, the electrical potential and current density distribution become more uniform with the introduction of 3D CNF scaffold (Figure 74h) and such unique characteristic might be ascribed to its enlarged specific area (dedicated by the numerous CNFs) and minimized Li nuclei size (contributed by its low nucleation overpotential). The uniformly distributed current density on the interface effectively avoids the tip effect observed on the planar $\mathrm{Cu}$ counterpart, ensuring more homogeneous $\mathrm{Li}$ adsorption to the overall electrode surface. Therefore, $\mathrm{Li}$ is 
evenly plated onto the CNF surface at the early stage and further extension of cycling duration did not trigger the growth of Li dendrite either (Figure 74i). Briefly, taking advantage of its low Li nucleation overpotential and homogeneously distributed current density, the CNF@Ni electrode could effectively erase the adverse influences of $\mathrm{Li}$ dendrites or other byproducts during the electrochemical deposition process and thus favor the highly reversible Li plating/stripping with satisfactory Coulombic efficiency.

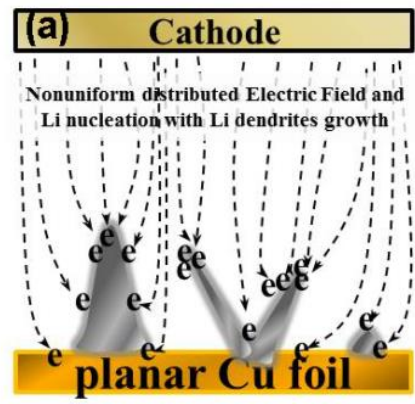

(d)

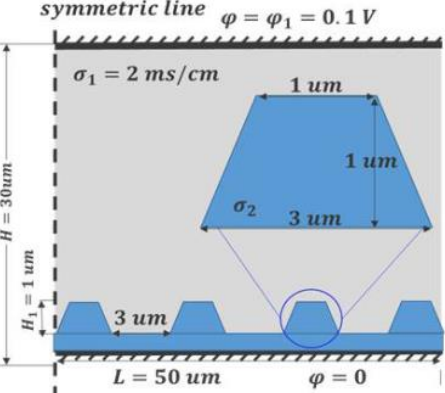

(g)

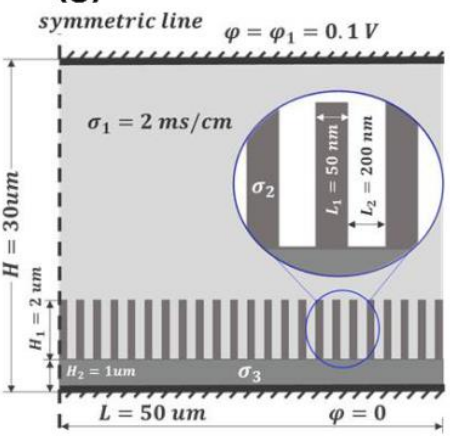

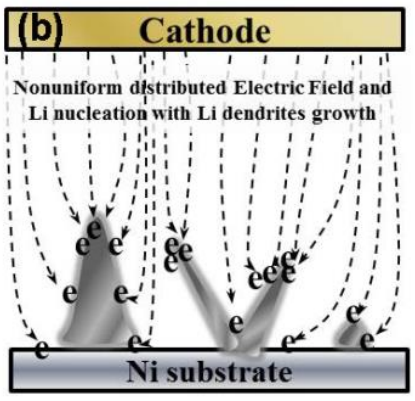

(e)

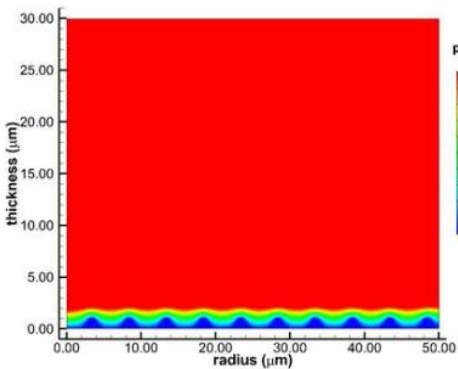

(h)
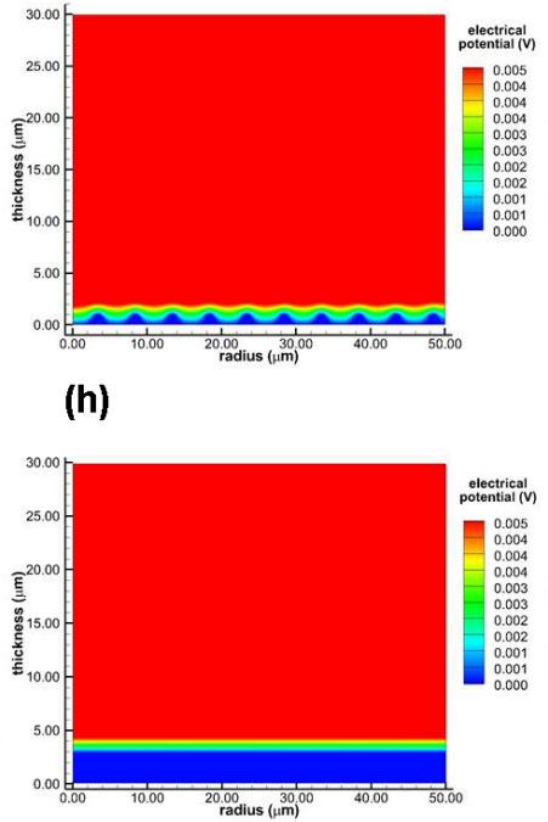

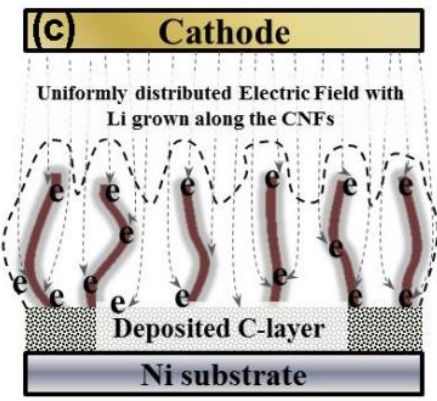

(f)

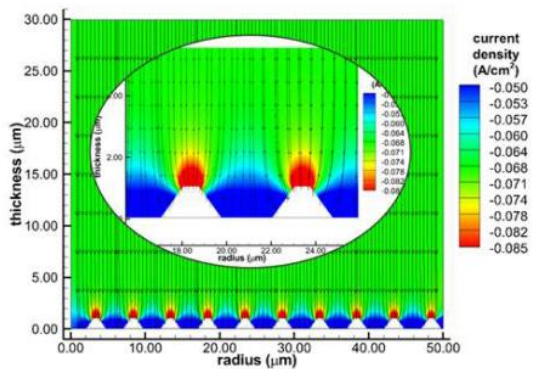

(i)

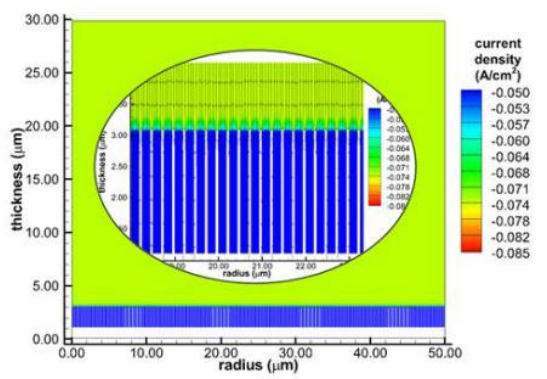

Figure 74 Illustration of the proposed electrochemical deposition processes of Li metal on planar $\mathrm{Cu}(\mathrm{a}), 3 \mathrm{D} \mathrm{Ni}$ foam (b), and 3D CNF@Ni foam (c), and models of the electric potential and current density distributions for a planar Cu electrode (d-f) and a CNF@Ni electrode (g-i). 


\subsection{Electrochemical performance of Full cells}

To verify the superiority of the $\mathrm{HEO}_{\mathrm{x}}-\mathrm{Li}$ ( $\mathrm{Li}$ metal foil with $\mathrm{HEO}$ protection layer) and CNF@Ni-Li foam (10 mAh cm${ }^{-2}$ deposition of Li) electrodes, full-cells were assembled with the corresponding electrochemical performance further evaluated. Typically, PEO-Li $\mathrm{L}_{0.5}$ coated $\mathrm{Li}$ foils were paired with commercial $\mathrm{LiCoO}_{2}$ with the control sample using pure $\mathrm{Li}$ foil, while CNF@Ni foam electrodes were employed as both sulfur and Li host to assemble the full cells (S/CNF-HC-Ni $\left.\mathrm{S}_{2} \| \mathrm{CNF} @ \mathrm{Ni}-\mathrm{Li}\right)$. As shown in Figure 75a, the $\mathrm{Li}_{0.5}|| \mathrm{LiCoO}_{2}$ cell has a higher and more stable capacity than the $\mathrm{Li}|| \mathrm{LiCoO}_{2}$ cell at the current density of $1 \mathrm{C}\left(1 \mathrm{C}=140 \mathrm{mAh} \mathrm{g}{ }^{-}\right.$ $\left.{ }^{1}\right)$. Besides, the Coulombic efficiency of $\mathrm{Li}_{0.5}|| \mathrm{LiCoO}_{2}$ battery is also much higher than that of $\mathrm{Li} \| \mathrm{LiCoO}_{2}$ battery. The corresponding charge-discharge curves of $\mathrm{Li} \| \mathrm{LiCoO}_{2}$ and $\mathrm{Li}_{0.5}|| \mathrm{LiCoO}_{2}$ batteries are shown in Figure $75 \mathrm{~b}$ and c, respectively. As illustrated, the discharge capacity of $\mathrm{Li} \| \mathrm{LiCoO}_{2}$ battery significantly decreases after 10 cycles (Figure 75b). By contrast, the chargedischarge curves of $\mathrm{Li}_{0.5} \| \mathrm{LiCoO}_{2}$ battery are much overlapped, indicating a much stable and reversible capacity (Figure 75c).

To further evaluate this "hairy foam" host in high energy storage for practical applications, the electrochemical performances of "Li-S" full cells were investigated by employing the "hairy foam" both as sulfur and lithium host, as schemed in Figure 75d. The detailed synthesis process can be found in Chapter 5. The working mechanism is illustrated in Figure 75e. As the sulfur host (S/CNF-HC-Ni $\left.\mathrm{S}_{2}\right)$, the 3D dual-carbon decorated Ni foam can significantly increase the electrons/ion kinetics, physically protect the polysulfides, and accommodate the volume change during the conversion reactions. ${ }^{[221]}$ Besides, the elaborately introduced "chemical anchors", such as "C-S", "Ni $\mathrm{N}_{2}$ ", are expected to provide strong chemical adsorption and accelerated electrocatalytic conversion towards polysulfides. ${ }^{[221]}$ As the lithium host (CNF@Ni-Li), the 
"hairy" foam can enable the stable Li stripping and plating electrochemistry by regulating the distribution of local curret density, reducing the nucleation overpotential, and accommodating the large volume change during the electrochemical process. Therefore, lithium can be uniformly deposited in the carbon matrix without forming any dendritic Li on the anode. Take advantages both from the cathode and anode sides, the electrochemical performance of "Li-S" full cells can be comprehensively improved. The superior rate performance of the "Li-S" full cell was evaluated at different current densities, as displayed in Figure 75f. The sulfur loading in the cathode is $3.58 \mathrm{mg} \mathrm{cm}^{-2}$. The specific capacities decrease with increasing the charge/discharge rates. The average reversible capacities of the full cell can be delivered at $\sim 1200, \sim 800, \sim 750$, and $\sim 650 \mathrm{mAh} \mathrm{g}^{-1}$ at $0.2,0.5,1$, and $2 \mathrm{C}$, respectively. Even at a much higher current density of $5 \mathrm{C}$, the full cell can still retain an average capacity of $\sim 500 \mathrm{mAh} \mathrm{g}^{-1}$. When the current densities were step by step switched back to $0.2 \mathrm{C}$, all the specific capacity can be recovered to the previous corresponding stages, demonstrating the excellent electrode stability and fast electrons/ion kinetics. Besides, the S/CNF-HC-Ni ${ }_{3} \mathrm{~S}_{2} \| \mathrm{CNF} @ \mathrm{Ni}-\mathrm{Li}$ full cell cycled at $0.1 \mathrm{C}(1 \mathrm{C}=$ $1000 \mathrm{~mA} \mathrm{~g}^{-1}$ ) can maintain a high specific capacity ( $\left.c a .900 \mathrm{mAh} \mathrm{g}^{-1}\right)$ with the Coulombic efficiency close to $100 \%$ after 100 cycles (Figure $75 \mathrm{~g}$ ), showing relatively higher capacity retention than the control cell with same sulfur electrode but paired with pure $\mathrm{Li}$ foil as the anode. More remarkably, the coupled full cell can maintain a capacity of $\sim 400 \mathrm{mAh} \mathrm{g}^{-1}$ (Figure $75 \mathrm{~h}$ ) even at $5 \mathrm{C}$ after 800 cycles with Coulombic efficiency close to $100 \%$, showing significantly improved long-term cycle stability compared with our previous work ${ }^{[221]}$ and reported literature (Table 9). Such comprehensively improved electrochemical performance demonstrates the great promise of this "hairy" foam to enable the high energy storage systems for practical applications. 

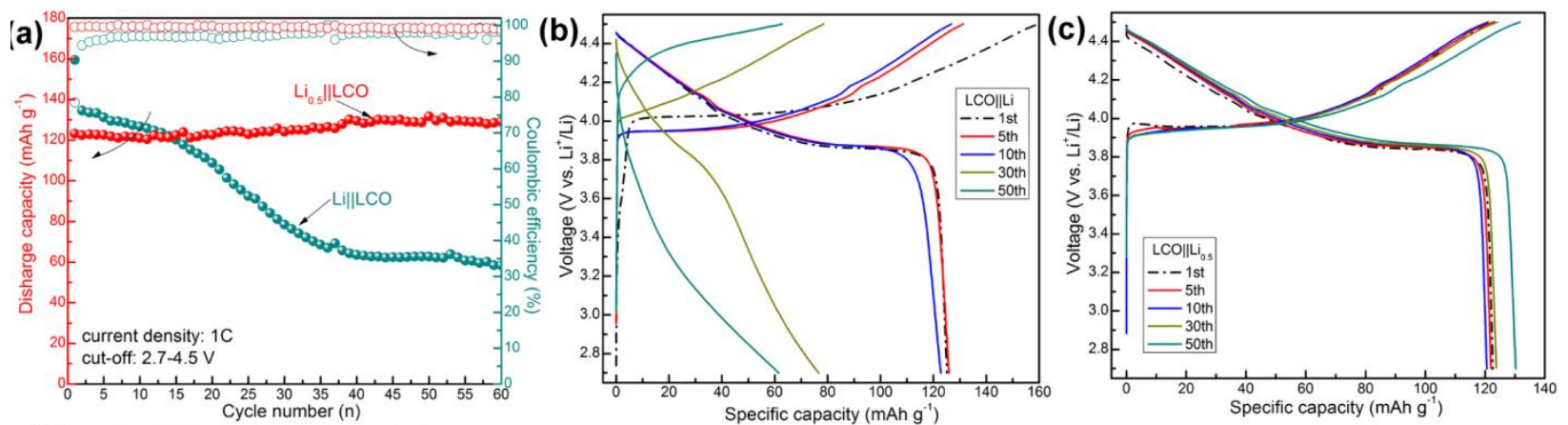

(d)

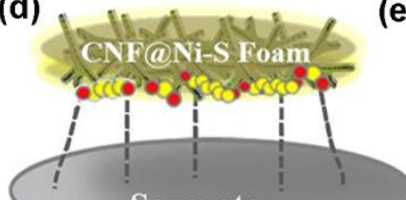

Separator

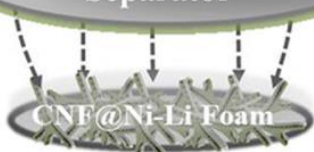

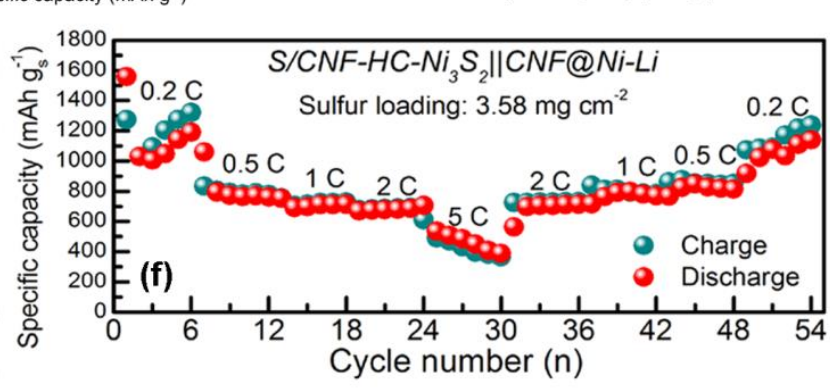
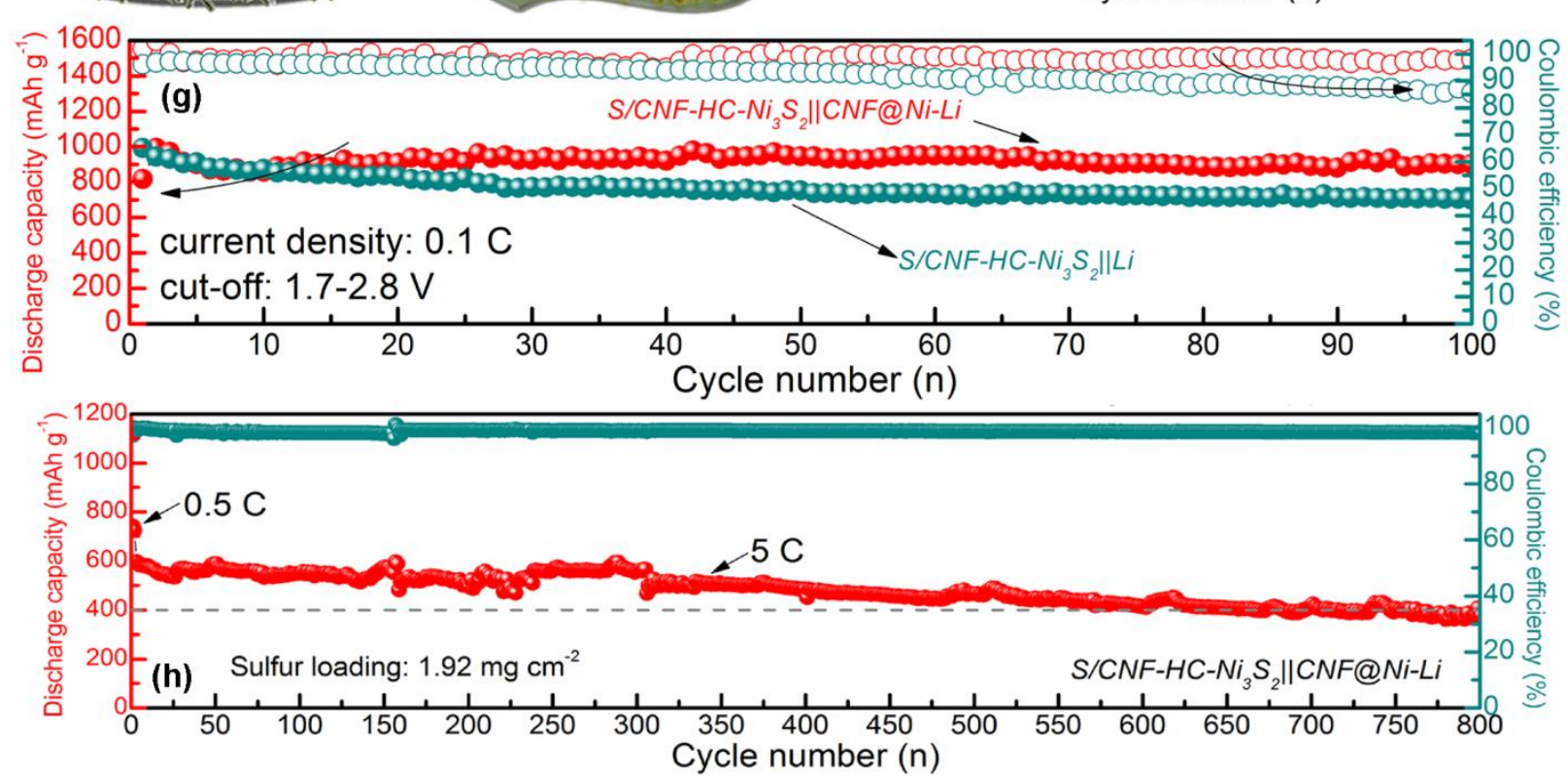

Figure 75 (a) cycle performance of $\mathrm{Li}_{0.5}|| \mathrm{LCO}$ and $\mathrm{Li}|| \mathrm{LCO}$ full cells with corresponding charge-

discharge curves in (b, c), respectively. (d) Schemed structure of "Li-S" full cell by using CNF@Ni foam both as sulfur and lithium host with the corresponding schemed working mechanism (e). Rate capability (f) and cycle performance of "Li-S" full cells at the current density of $0.1 \mathrm{C}(\mathrm{g})$ and $5 \mathrm{C}(\mathrm{h})$. 
Table 9 Performance comparison of S/CNF-HC-Ni $\mathrm{S}_{2} \| \mathrm{CNF} @ \mathrm{Ni}-\mathrm{Li}$ with reported cathodes.

\begin{tabular}{|c|c|c|c|c|c|}
\hline $\begin{array}{l}\text { Cathodic } \\
\text { materials }\end{array}$ & $\begin{array}{l}\text { Mass loading } \\
\quad\left(\mathrm{mg} \mathrm{cm}^{-2}\right)\end{array}$ & Rate & $\begin{array}{l}\text { Capacity (cycles) } \\
\quad\left(\mathrm{mAh} \mathrm{g}^{-1}\right)\end{array}$ & $\begin{array}{c}\text { Areal } \\
\text { capacity } \\
\left(\mathbf{m A h} \mathbf{~ c m}^{-2}\right)\end{array}$ & Reference \\
\hline \multirow{4}{*}{$\begin{array}{c}\text { S/CNF-HC- } \\
\mathrm{Ni}_{3} \mathrm{~S}_{2} \| \mathrm{CNF} @ \mathrm{Ni}-\mathrm{Li}\end{array}$} & 2.10 & $0.1 \mathrm{C}$ & 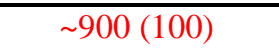 & 1.89 & \multirow{4}{*}{ This work } \\
\hline & 2.32 & $1 \mathrm{C}$ & $\sim 850(200)$ & 1.97 & \\
\hline & 2.78 & $3 \mathrm{C}$ & $\sim 600(200)$ & 1.67 & \\
\hline & 1.92 & $5 \mathrm{C}$ & $\sim 400(800)$ & 0.77 & \\
\hline \multirow{4}{*}{$\mathrm{S} / \mathrm{CNF}-\mathrm{HC}-\mathrm{Ni}_{3} \mathrm{~S}_{2} \| \mathrm{Li}$} & 2.31 & $0.2 \mathrm{~A} \mathrm{~g}^{-1}$ & $850(100)$ & 1.97 & \multirow{4}{*}[221]{} \\
\hline & 2.13 & $2 \mathrm{Ag}^{-1}$ & $620(300)$ & 1.32 & \\
\hline & 2.20 & $5 \mathrm{~A} \mathrm{~g}^{-1}$ & $\sim 400(450)$ & 0.88 & \\
\hline & 4.11 & $0.2 \mathrm{~A} \mathrm{~g}^{-1}$ & $760(100)$ & 3.12 & \\
\hline \multirow{3}{*}{ NDHC@C-S } & 1.2 & $0.5 \mathrm{C}$ & $813(250)$ & 0.98 & \multirow{3}{*}[222]{} \\
\hline & 2 & $0.5 \mathrm{C}$ & $\sim 700(250)$ & 1.40 & \\
\hline & 4 & $0.5 \mathrm{C}$ & $400(500)$ & 1.60 & \\
\hline \multirow{2}{*}{$\mathrm{S} / \mathrm{Fe}_{3} \mathrm{O}_{4} @ \mathrm{C}-\mathrm{G}$} & \multirow[b]{2}{*}{2} & $0.2 \mathrm{C}$ & $1102(100)$ & 2.2 & \multirow{2}{*}{ [223] } \\
\hline & & $0.5 \mathrm{C}$ & $892(500)$ & 1.8 & \\
\hline \multirow{2}{*}{ SAS } & \multirow{2}{*}{$0.8-1.1$} & $1 \mathrm{C}$ & $710(200)$ & $\begin{array}{l}\sim 0.71 \\
\end{array}$ & \multirow{2}{*}{ [224] } \\
\hline & & $2 \mathrm{C}$ & $605(300)$ & $\sim 0.61$ & \\
\hline \multirow{2}{*}{$\mathrm{V}_{2} \mathrm{O}_{5}-\mathrm{S}-\mathrm{CNG}$} & \multirow{2}{*}{1.2} & $0.1 \mathrm{C}$ & $838(200)$ & 1.0 & \multirow{2}{*}[225]{} \\
\hline & & $0.5 \mathrm{C}$ & $713(150)$ & 0.86 & \\
\hline C-SCH & 1.2 & $1000 \mathrm{~mA} \mathrm{~g}^{-1}$ & $500(100)$ & 0.6 & [226] \\
\hline Li-Mg/S & 2 & $0.1 \mathrm{C}$ & $606(200)$ & 1.2 & [227] \\
\hline \multirow{2}{*}{$\mathrm{S} / \mathrm{CoOOH}$} & \multirow{2}{*}{$1.0-1.3$} & $0.2 \mathrm{C}$ & $788(100)$ & $\sim 0.8$ & \multirow{2}{*}{ [228] } \\
\hline & & $1 \mathrm{C}$ & $376(500)$ & $\sim 0.4$ & \\
\hline \multirow{2}{*}{ B/2D MOF-Co } & \multirow{2}{*}{1.5} & $0.5 \mathrm{C}$ & $700(200)$ & 1.05 & \multirow{2}{*}{ [229] } \\
\hline & & $1 \mathrm{C}$ & $450(600)$ & 0.68 & \\
\hline \multirow{2}{*}{$\mathrm{S} / \mathrm{NiCo}_{2} \mathrm{O}_{4}$} & $13-15$ & $0.5 \mathrm{C}$ & $646(400)$ & $\sim 0.97$ & [230] \\
\hline & $1.3-1.5$ & $1 \mathrm{C}$ & $750(500)$ & 1.12 & {$[\angle 20]$} \\
\hline & 3 & & $907(300)$ & 2.7 & \\
\hline $3 \mathrm{WO}_{3}: 1 \mathrm{WS}_{2}$ & 5 & $0.5 \mathrm{C}$ & $765(300)$ & 3.82 & {$[231]$} \\
\hline & 10 & & $\sim 500(250)$ & $\sim 5$ & \\
\hline $\mathrm{Fe}_{1-\mathrm{x}} \mathrm{S}-\mathrm{NC}-\mathrm{S}$ & & & $1064(200)$ & 1.06 & [232] \\
\hline S/N/C-S & 1 & $0.5 \mathrm{C}$ & $547(200)$ & 0.55 & {$[232]$} \\
\hline TI-NHCNS9/S & 1.2 & $1 \mathrm{C}$ & $\sim 600(600)$ & 0.72 & [233] \\
\hline & & $0.2 \mathrm{C}$ & $1000(200)$ & 1.5 & \\
\hline $\mathrm{DS} / \mathrm{rCO}$ & 15 & $0.5 \mathrm{C}$ & $950(200)$ & 1.42 & [234] \\
\hline PS/TGO & 1.5 & $1.0 \mathrm{C}$ & $900(200)$ & 1.35 & {$[234]$} \\
\hline & & $2.0 \mathrm{C}$ & $900(200)$ & 1.35 & \\
\hline & 1.2 & $0.5 \mathrm{C}$ & $\begin{array}{l}\sim 600(500) \\
\end{array}$ & 0.72 & \\
\hline TOE with CS & 4 & $0.02 \mathrm{C}$ & $\sim 750(65)$ & 3 & {$[235]$} \\
\hline & & $0.2 \mathrm{C}$ & $896(200)$ & $\sim 1.6$ & [236] \\
\hline SDCD & $1.5-1.8$ & $0.5 \mathrm{C}$ & $836(200)$ & $\sim 1.5$ & {$[236]$} \\
\hline n-hC@S & 2.5 & $0.1 \mathrm{C}$ & $800(650)$ & 2.0 & [237] \\
\hline 政- & 2.5 & $1 \mathrm{C}$ & $350(900)$ & 0.88 & {$[23 /]$} \\
\hline $\mathrm{Mo}_{2} \mathrm{C}-\mathrm{C}$ NOs@S & 1.1 & $1 \mathrm{C}$ & $762(600)$ & 0.84 & [238] \\
\hline $\mathrm{Fe}_{2} \mathrm{O} / \mathrm{C} / \mathrm{S}$ & 10 & $0.2 \mathrm{C}$ & $765(50)$ & 0.76 & [239] \\
\hline $\mathrm{Fe}_{3} \mathrm{O}_{4} / \mathrm{C} / \mathrm{s}$ & 1.0 & $1 \mathrm{C}$ & $642(300)$ & 0.64 & {$[259]$} \\
\hline S/RuO_-15 & $1.3-1.5$ & $0.2 \mathrm{C}$ & $788(100)$ & 1.2 & {$[240]$} \\
\hline $\mathrm{S} / \mathrm{K} U \mathrm{O}_{\mathbf{2}^{-13}}$ & $1.5-1.5$ & $1.0 \mathrm{C}$ & $500(500)$ & 0.75 & {$[240]$} \\
\hline $\mathrm{Co}-\mathrm{NbN} / \mathrm{rGO} / \mathrm{S}$ & $1.2-1.7$ & $0.2 \mathrm{C}$ & $706(120)$ & 1.2 & [241] \\
\hline
\end{tabular}




\subsection{Conclusions}

In conclusion, we have developed two methods to modify the Li metal anodes, i.e., by coating

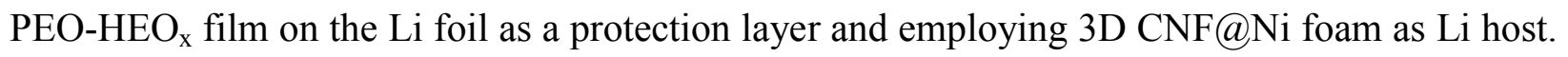
The PEO-HEO ${ }_{x}$ hybrid film can mechanically prevent the Li dendrites growth due to due to the ceramic oxides, and more importantly, regulate uniform $\mathrm{Li}^{+}$flux flow on the interface, thus can achieve the uniform nucleation and deposition of $\mathrm{Li}$ beneath the PEO-HEO film. Furthermore, the advantages of 3D CNF@Ni foam with a large specific surface area as a Li host has also been demonstrated. The large surface area with porous $\mathrm{Ni}$ foam skeleton can effectively reduce current density, enable uniform distribution of the electric field, thereby inhibiting the growth of Li dendrites and reducing the volume change during charge and discharge. As such, the dendritefree modified Li metal anodes (PEO-HEOx coated Li and CNF@Ni-Li), have a high CE ( 98\%) and an ultralong lifespan $(\sim 1000 \mathrm{~h})$. The electrochemical performances of both the corresponding half cells and symmetric cells demonstrate the significantly improved Li stripping and plating electrochemistry. Moreover, high capacity with excellent cycle stability can be achieved from the paired full cells $\left(\mathrm{Li}_{0.5}|| \mathrm{LiCoO}_{2}\right.$ and S/CNF-HC-Ni $\left.\mathrm{S}_{2}|| \mathrm{CNF} @ \mathrm{Ni}-\mathrm{Li}\right)$. Remarkably, the S/CNFHC-Ni $\mathrm{S}_{2} \| \mathrm{CNF} @ \mathrm{Ni}-\mathrm{Li}$ full battery show excellent cycle stability and high Coulombic efficiency by using CNF modified Ni foam as both sulfur and Li host. As to the cathode side, the polysulfides can be significantly suppressed due to the physical barrier protection and "chemical anchors" interaction; for the anode side, the 3D CNF@Ni enables a stable and uniform Li stripping/plating electrochemistry. Therefore, the electrochemical performance of the corresponding "Li-S" battery can be comprehensively improved. Overall, these works provide a simple but effective strategy to achieve uniform metal anode deposition by introducing a coating layer as artificial SEI layer and employing a 3D conductive framework both as sulfur and Li host. 


\section{Chapter 7: Future work and implications}

In this work, we have successfully developed the sulfur electrodes (FeS $\mathrm{F}_{2} \mathrm{C}-\mathrm{S}$ and S/CNFHC-Ni $\mathrm{S}_{2}$ ) with excellent cycle stability and Li metal anodes with highly stable stripping/plating electrochemistry (PEO-HEO coated Li foil and 3D CNF@Ni-Li foam). By optimizing the microstructure and synthesize technology for both the sulfur cathodes and Li metal anodes, the electrochemical performance of "Li-S" batteries can be comprehensively improved. However, to further increase the utilization of sulfur and the energy/power density, some issues still need to be addressed in future work:

\section{FeS $@$ @-S electrode:}

(1) Optimize the size of the hollow space in the carbon nanotube to increase the fast $\mathrm{Li}^{+}$ diffusion and provide a more efficient buffer mechanism;

(2) Adjust the CVD carbon deposition time to obtain thin but protective carbon shell, thus can reduce the mass of inactive materials; the thin carbon shell can also enable a much easier sulfur diffusion into the host;

(3) Optimize the particle size of nano $\mathrm{FeS}_{2}$ in the void space of the carbon shell. This might be realized by adjusting the heating rate, the percentage of $\mathrm{H}_{2}$ in Ar, et al. The nanosized $\mathrm{FeS}_{2}$ with the large surface area can provide more efficient catalytic process during the polysulfide conversion;

\section{S/CNF-HC-Ni $\mathrm{S}_{2}$ electrode:}

(1) Using toluene as the carbon precursor needs high temperature $\left(800{ }^{\circ} \mathrm{C}\right)$ for pyrolysis, which will damage the mechanical stability of Ni foam skeleton; other precursors can be taken into consideration, such as ethylene, which can be pyrolyzed at $\sim 450{ }^{\circ} \mathrm{C}$. 
(2) Adjust the CVD carbon deposition time to obtain thin but protective carbon shell, thus can reduce the mass of inactive materials; the thin carbon shell can also enable a much easier sulfur diffusion into the host;

(3) The Ni foam will bring additional weight and affect the energy density of the whole battery device. In the future, a relatively solid yet lightweight substrate or current collector should be employed to achieve high energy densities and sulfur loadings, promoted kinetics and long lifetime, simultaneously, for practical applications;

\section{PEO-HEO protection layer on Li metal anode:}

(1) The structure and potential properties of HEO materials still have much to be explored, such as the uniform $\mathrm{Li}^{+}$flux across the PEO-HEO film;

(2) The thickness of the PEO-HEO film should be optimized. This might be realized by adjusting the viscosity of the slurry, spin coating;

(3) More characterization can be conducted to confirm that Li deposited beneath the PEOHEO protection layer;

(4) A model with integrating this "artificial SEI (PEO-HEO film)" might be established to further understand the protection mechanism.

\section{DCNF@Ni as Li host:}

(1) Carbon loading on the Ni foam need to adjust when using as a Li host to minimize the capacity contribution from the lithiated graphitic carbon;

(2) Electrochemical performances of S/CNF-HC-Ni $\mathrm{S}_{2}|| \mathrm{CNF} @ \mathrm{Ni}-\mathrm{Li}$ full cells with higher sulfur loading need to be evaluated, such as long-term cycle stability and rate capability;

(3) Deep charge-discharge cycle stability could be investigated at the various current density and constant capacity $\left(\geq 5 \mathrm{mAh} \mathrm{cm}^{-2}\right)$. As the large surface area of the 
CNF@Ni host, it can significantly reduce the local current density, and therefore excellent cycle stability at deep charge-discharge conditions;

(4) A model illustrating the distribution of $\mathrm{Li}$ ion in the 3D CNF@Ni foam electrode combined with the electric field distribution might be established to further understand the deposition mechanism; Models can be established by changing the current densities, initial morphology of Li seeds;

As a summary of this project, we have done a sufficient literature review and obtained deep insight into the "Li-S" battery system. Besides, the sulfur electrodes with high cycle stability and rate capability have been successfully designed. The structure advantages which can physically and chemically adsorb the polysulfides have also been confirmed by different characterization methods. Furthermore, we developed PEO-HEO film protected Li foil and 3D CNF@Ni-Li electrodes to deliver significantly improved stability of Li stripping/plating. With this in mind, we hope to provide an in-depth understanding and offer avenues in the rational design of Li-S batteries with long cycle life and high energy/power density in the near future. 


\section{Chapter 8: Summary and Perspectives}

In this research, we provide a broad overview of the recent approaches utilized to chemically bind the LiPSs. Different types of conductive host materials have been reviewed. By surface modifications (heteroatoms doping, amphiphilic polymers, TMOs/TMSs polar sites, and $\mathrm{MOF}),-\mathrm{S}_{\mathrm{n}}-$ species are chemically grafted to the surface functional groups or polymer backbones. With the help of chemical bonds, significant electrochemical performances can be obtained in terms of cycle stability, rate capability, and Coulombic efficiency. The mechanistic understanding gained by theoretical calculations is also systematically summarized here, which broaden our insight on the role of this chemical bonding effect from the view of the molecular structure. Although significant progress has been achieved, the drawbacks of these "chemically anchored" sulfur composites still exist as summarized in each section. The "chemical anchor" is just a subdiscipline appealing to be explored and deeper understood. To realize the practical application of Li-S batteries, several factors concerning the best performance based on the "chemical anchor" need to be highly considered. Besides, the performance of the Li-S battery is a result of the integrative system, which demands multidimensional exploration including all the important components. Here comes to some perspective in the development of the Li-S battery field based on the "chemical anchor" mechanism as follows:

(1) Understanding the mechanism of Li-S battery both experimentally and theoretically

The mechanism of Li-S battery is much complicated as we discussed above. Until now, it is still not been fully understood. Long-chain polysulfides are highly soluble in the electrolyte, however, short-chain polysulfides with low electronic conductivity results in low kinetics of conversion. To better investigate this mechanism and explore the possible dominated factors influence the performance, both of the new characterization methods and theoretical calculation 
models need to be developed. Novel characterization methods may help to accurately identify the $-\mathrm{S}_{\mathrm{n}^{-}}$species at different states of discharge. The theoretical calculation can predict the behavior mechanism between the host materials and polysulfides. For example, if $\mathrm{Li}_{2} \mathrm{~S}_{8}$ and $\mathrm{Li}_{2} \mathrm{~S}_{6}$ can be identified at which plateau they are formed by novel characterization methods, and the theoretical models can predict which host material is most active at this plateau and provides the strongest chemical bonding, the polysulfides could be significantly under controlled. The current published literature shows various binding energy between different host materials and sulfur species, making it difficult to distinguish the best among all the candidates. A systematic theoretical calculation probably needs to be built with respect to the binding energy between the host materials and various polysulfides. These fundamental research are hoped to provide precise guidelines for the design of Li-S batteries. Besides, other important factors such as morphology, pore size, surface area are also needed to be taken into consideration during the design of the electrode as well as the calculation models.

(2) Optimizing the morphology and structure of sulfur electrodes

One thing that needs to be addressed is that the sulfur loading on the electrode should be at least larger than $2 \mathrm{mg} \mathrm{cm}^{-2}$ to possibly make its greatest advantages. As to the porous carbonaceous materials, heteroatoms doping may still be one of the most effective ways to create the polar-polar interaction on the polysulfides. Structures with large surface areas, such as 3D micro-/mesoporous carbon, metal foam, and layer-by-layer electrodes will be further explored to provide more doping sites as "chemical anchor". Different parameters (temperature, S/C mass ratio, concentration) during the synthesis process may need to be adjusted to obtain the best sulfur loading and satisfied chemical anchor sites. Short sulfur chains are also demonstrated to play an effective role in the adsorption of polysulfides during the lithiation process. Since no 
dissolving polysulfides are required, this kind of sulfur electrode can be applied in the carbonated electrolyte and increase the cycle stability. Sulfur chains covalently bonded to the polymer backbone should also shape the future of the energy storage. The flexible structure of the polymer backbone makes it possible to design different configurations in cell engineering. The challenge is to increase the sulfur loading by novel techniques, i.e., the degree of polymerization. As to the metal-based host materials, more attention should be paid to optimize the structure of the composites. Due to the intrinsic obstacles as mentioned in section 4.4 , metal oxides/sulfides usually act as additives and need to be composited with more conductive carbonaceous materials or polymer to either increase the conductivity or surface area. In addition, nano-sized porous or hierarchical structured metal-based host materials are starving for development, which is much important to facilitate the kinetics of the conversion process during the charging process.

\section{(3) Exploring appropriate electrolytes}

Electrolyte seems to be even more important but usually being neglected. Since the polysulfide dissolution and shuttle effect are much related to the properties of electrolytes, it is very emergency to explore appropriate electrolytes as well as the corresponding additives. Although some novel solvents or additives in liquid-based electrolytes, breakthroughs in solid or gel electrolytes have been proposed, challenges remain for the practical application in the future. Different electrolyte systems need to be explored to balance the advantages and disadvantages to achieve the superior performance of Li-S cells. For liquid electrolyte systems, the concentrations of the lithium salt, the electrolyte-to-sulfur ratio, the compatibility of additives need to be optimized to balance the reaction kinetics of polysulfides, and overall energy density. The feasible approaches for electrolyte modification may also be explored by forming self-inhibiting 
interlayers on both of the cathode and anode surfaces. The in situ formed thin film can effectively balance the low volumetric energy by introducing artificial interlayer or coating membrane.

(4) Based on the chemical anchor mechanism, Functional groups modified separators or interlayers are efficient in controlling the shuttle, redox of polysulfides. However, as we mentioned before, such an extra layer should not take expense in sacrificing total specific energy/power density due to the increasing volume and weight. Research into the functional separator/interlayer systems should also be regarding both of the interfaces on the cathode/separator and separator/anode. As the mechanism on each side of electrodes is different, advanced fabrication methods are starved for exploring to meet the distinct requirements from the two sides. The design of integrated cathode/separator or anode/separator electrodes with functional groups may also be an alternative way to stabilize the system without sacrificing the volumetric energy.

In summary, the system of $\mathrm{Li}-\mathrm{S}$ is such complex that many factors can influence the performance. Other solutions against the polysulfides may also include employing solid-state electrolyte, lithium metal anode protection but not based on the "chemical anchor" mechanism, so these will not be discussed in detail in this review. Based on these "chemical anchor" in this review, we hope to guide future development in the field and can enlighten discussion and provide directions for designing durable and high-performance Li-S batteries in the near future. 


\section{References}

[1] a) L. Qie, C. Zu, A. Manthiram, Adv. Energy Mater. 2016, 6, 1502459; b) B. Papandrea, X. Xu, Y. Xu, C.-Y. Chen, Z. Lin, G. Wang, Y. Luo, M. Liu, Y. Huang, L. Mai, X. Duan, Nano Res. 2016, 9, 240; c) R. Carter, L. Oakes, N. Muralidharan, C. L. Pint, J. Phys. Chem. C 2017, 121, 7718; d) G. Zhang, Z.-W. Zhang, H.-J. Peng, J.-Q. Huang, Q. Zhang, Small Methods 2017, 1, 1700134; e) Z. Zhang, L.-L. Kong, S. Liu, G.-R. Li, X.-P. Gao, Adv. Energy Mater. 2017, 7, 1602543.

[2] P. G. Bruce, S. A. Freunberger, L. J. Hardwick, J.-M. Tarascon, Nat. Mater. 2012, 11, 19.

[3] X. Ji, K. T. Lee, L. F. Nazar, Nat. Mater. 2009, 8, 500.

[4] N. Brun, K. Sakaushi, L. Yu, L. Giebeler, J. Eckert, M. M. Titirici, Phys. Chem. Chem. Phys. 2013, 15, 6080.

[5] a) J. P. Perdew, K. Burke, M. Ernzerhof, Phys. Rev. Lett. 1996, 77, 3865; b) S. Grimme, J. Comput. Chem. 2006, 27, 1787; c) K. E. Riley, M. Pitoňák, P. Jurečka, P. Hobza, Chem. Rev. 2010, 110, 5023.

[6] D. Hohl, R. O. Jones, R. Car, M. Parrinello, J. Chem. Phys. 1988, 89, 6823.

[7] a) Y. V. Mikhaylik, J. R. Akridge, J. Electrochem. Soc. 2004, 151, A1969; b) R. D. Rauh, K. M. Abraham, G. F. Pearson, J. K. Surprenant, S. B. Brummer, J. Electrochem. Soc. 1979, 126, 523; c) E. Peled, Y. Sternberg, A. Gorenshtein, Y. Lavi, J. Electrochem. Soc. 1989, 136, 1621; d) J. Shim, K. A. Striebel, E. J. Cairns, J. Electrochem. Soc. 2002, 149, A1321; e) Y. V. Mikhaylik, J. R. Akridge, J. Electrochem. Soc. 2003, 150, A306.

[8] S.-E. Cheon, K.-S. Ko, J.-H. Cho, S.-W. Kim, E.-Y. Chin, H.-T. Kim, J. Electrochem. Soc. 2003, 150, A796. 
[9] M. Wild, L. O'Neill, T. Zhang, R. Purkayastha, G. Minton, M. Marinescu, G. J. Offer, Energy Environ. Sci. 2015, 8, 3477.

[10] S. Xin, L. Gu, N.-H. Zhao, Y.-X. Yin, L.-J. Zhou, Y.-G. Guo, L.-J. Wan, J. Am. Chem. Soc 2012, 134, 18510.

[11] X.-B. Cheng, R. Zhang, C.-Z. Zhao, Q. Zhang, Chem. Rev. 2017, 117, 10403.

[12] a) B. Zhang, X. Qin, G. R. Li, X. P. Gao, Energy Environ. Sci. 2010, 3, 1531; b) Y.-S. Su, A. Manthiram, Electrochimi. Acta 2012, 77, 272; c) J. L. Wang, J. Yang, J. Y. Xie, N. X. Xu, Y. Li, Electrochem. Commun. 2002, 4, 499; d) B. Zhang, C. Lai, Z. Zhou, X. P. Gao, Electrochimi. Acta 2009, 54, 3708; e) Y. Fu, A. Manthiram, J. Phys. Chem. C 2012, 116, 8910; f) J. Yan, X. Liu, H. Qi, W. Li, Y. Zhou, M. Yao, B. Li, Chem. Mater. 2015, $27,6394$.

[13] Z. Li, H. B. Wu, X. W. Lou, Energy Environ. Sci. 2016, 9, 3061.

[14] a) C. Lai, X. P. Gao, B. Zhang, T. Y. Yan, Z. Zhou, J. Phys. Chem. C 2009, 113, 4712; b) S. Li, M. Xie, J. Liu, H. Wang, H. Yan, Electrochem. Solid-State Lett. 2011, 14, A105; c) J. Guo, Y. Xu, C. Wang, Nano Lett. 2011, 11, 4288.

[15] J. F. Mike, J. L. Lutkenhaus, J. Polym. Sci. Part B: Polym. Phys. 2013, 51, 468.

[16] Q. Sun, B. He, X.-Q. Zhang, A.-H. Lu, ACS Nano 2015, 9, 8504.

[17] K. Mi, Y. Jiang, J. Feng, Y. Qian, S. Xiong, Adv. Funct. Mater. 2016, 26, 1571.

[18] S. Lu, Y. Cheng, X. Wu, J. Liu, Nano Lett. 2013, 13, 2485.

[19] a) H. Wang, Y. Yang, Y. Liang, J. T. Robinson, Y. Li, A. Jackson, Y. Cui, H. Dai, Nano Lett. 2011, 11, 2644; b) R. Chen, T. Zhao, J. Lu, F. Wu, L. Li, J. Chen, G. Tan, Y. Ye, K. Amine, Nano Lett. 2013, 13, 4642. 
[20] a) G. Zheng, Y. Yang, J. J. Cha, S. S. Hong, Y. Cui, Nano Lett. 2011, 11, 4462; b) M.-Q. Zhao, X.-F. Liu, Q. Zhang, G.-L. Tian, J.-Q. Huang, W. Zhu, F. Wei, ACS nano 2012, 6, 10759.

[21] J. Zhang, C.-P. Yang, Y.-X. Yin, L.-J. Wan, Y.-G. Guo, Adv. Mater. 2016, 28, 9539.

[22] a) J. Wang, J. Yang, J. Xie, N. Xu, Adv. Mater. 2002, 14, 963; b) D. Chaudhuri, A. Kumar, I. Rudra, D. D. Sarma, Adv. Mater. 2001, 13, 1548; c) Y. Lu, Y. Yang, A. Sellinger, M. Lu, J. Huang, H. Fan, R. Haddad, G. Lopez, A. R. Burns, D. Y. Sasaki, J. Shelnutt, C. J. Brinker, Nature 2001, 410, 913.

[23] Y. Fu, A. Manthiram, RSC Adv. 2012, 2, 5927.

[24] J. H. Kim, K. Fu, J. Choi, K. Kil, J. Kim, X. Han, L. Hu, U. Paik, Sci. Rep. 2015, 5, 8946.

[25] H. Chen, W. Dong, J. Ge, C. Wang, X. Wu, W. Lu, L. Chen, Sci. Rep. 2013, 3, 1910.

[26] W. Zhou, Y. Yu, H. Chen, F. J. DiSalvo, H. D. Abruña, J. Am. Chem. Soc 2013, 135, 16736.

[27] a) S. Rehman, S. Guo, Y. Hou, Adv. Mater. 2016, 28, 3167; b) P. Wei, M. Fan, H. Chen, D. Chen, C. Li, K. Shu, C. Lv, Int. J. Hydrogen Energy 2016, 41, 1819.

[28] a) H.-C. Wang, C.-Y. Fan, Y.-P. Zeng, X.-H. Zhang, W.-H. Li, S.-Y. Liu, H.-Z. Sun, J. Zhang, L.-N. Sun, X.-L. Wu, Chem. Eur. J. 23, 9666; b) X. He, H. Hou, X. Yuan, L. Huang, J. Hu, B. Liu, J. Xu, J. Xie, J. Yang, S. Liang, X. Wu, Sci. Rep. 2017, 7, 40679.

[29] a) S. A. Ahad, P. Ragupathy, S. Ryu, H.-W. Lee, D. K. Kim, Chem. Commun. 2017, DOI: 10.1039/C7CC04229A; b) X. Liang, L. F. Nazar, ACS Nano 2016, 10, 4192.

[30] S.-H. Chung, A. Manthiram, Electrochimi. Acta 2013, 107, 569.

[31] M. Yan, Y. Zhang, Y. Li, Y. Huo, Y. Yu, C. Wang, J. Jin, L. Chen, T. Hasan, B. Wang, B.-L. Su, J. Mater. Chem. A 2016, 4, 9403. 
[32] Y. Yang, G. Yu, J. J. Cha, H. Wu, M. Vosgueritchian, Y. Yao, Z. Bao, Y. Cui, ACS Nano 2011, 5, 9187.

[33] Y. Dong, S. Liu, Z. Wang, Y. Liu, Z. Zhao, J. Qiu, Nanoscale 2015, 7, 7569.

[34] Y. Cao, X. Li, I. A. Aksay, J. Lemmon, Z. Nie, Z. Yang, J. Liu, Phys. Chem. Chem. Phys. 2011, 13,7660 .

[35] F. Wu, J. Li, Y. Su, J. Wang, W. Yang, N. Li, L. Chen, S. Chen, R. Chen, L. Bao, Nano Lett. 2016, 16, 5488.

[36] N. Jayaprakash, J. Shen, S. S. Moganty, A. Corona, L. A. Archer, Angew. Chem. 2011, $123,6026$.

[37] C. Zhang, H. B. Wu, C. Yuan, Z. Guo, X. W. Lou, Angew. Chem. 2012, 124, 9730.

[38] Y. Fu, A. Manthiram, Chem. Mater. 2012, 24, 3081.

[39] R. Elazari, G. Salitra, A. Garsuch, A. Panchenko, D. Aurbach, Adv. Mater. 2011, 23, 5641.

[40] W. Zhang, D. Qiao, J. Pan, Y. Cao, H. Yang, X. Ai, Electrochimi. Acta 2013, 87, 497.

[41] Q. Pang, X. Liang, C. Y. Kwok, L. F. Nazar, J. Electrochem. Soc. 2015, 162, A2567.

[42] S. Zheng, F. Yi, Z. Li, Y. Zhu, Y. Xu, C. Luo, J. Yang, C. Wang, Adv. Funct. Mater. 2014, 24, 4156.

[43] S. Zhang, Front. Energy Res. 2013, 1, 10.

[44] J.-S. Kim, T. H. Hwang, B. G. Kim, J. Min, J. W. Choi, Adv. Funct. Mater. 2014, 24, 5359.

[45] M. F. L. De Volder, S. H. Tawfick, R. H. Baughman, A. J. Hart, Science 2013, 339, 535.

[46] Q. Pang, L. F. Nazar, ACS Nano 2016, 10, 4111. 
[47] a) L. Zhang, L. Ji, P.-A. Glans, Y. Zhang, J. Zhu, J. Guo, Phys. Chem. Chem. Phys. 2012, 14, 13670; b) L. Ji, M. Rao, H. Zheng, L. Zhang, Y. Li, W. Duan, J. Guo, E. J. Cairns, Y. Zhang, J. Am. Chem. Soc 2011, 133, 18522; c) R. Demir-Cakan, M. Morcrette, F. Nouar, C. Davoisne, T. Devic, D. Gonbeau, R. Dominko, C. Serre, G. Férey, J.-M. Tarascon, J. Am. Chem. Soc 2011, 133, 16154; d) H. Al Salem, G. Babu, C. V. Rao, L. M. R. Arava, J. Am. Chem. Soc 2015, 137, 11542.

[48] J. Wang, L. Liu, Z. Ling, J. Yang, C. Wan, C. Jiang, Electrochimi. Acta 2003, 48, 1861.

[49] a) J. Wang, J. Yang, C. Wan, K. Du, J. Xie, N. Xu, Adv. Funct. Mater. 2003, 13, 487; b) X. M. He, L. Wang, W. H. Pu, J. G. Ren, W. Wu, C. Y. Jiang, C. R. Wan, J. Therm. Anal. Calorim. 2008, 94, 151.

[50] a) L. Xiao, Y. Cao, J. Xiao, B. Schwenzer, M. H. Engelhard, L. V. Saraf, Z. Nie, G. J. Exarhos, J. Liu, Adv. Mater. 2012, 24, 1176; b) J. Yan, B. Li, X. Liu, Nano Energy 2015, 18,245 .

[51] a) D. Han, B. Zhang, M. Xiao, P. Shen, S. Wang, G. Chen, Y. Meng, Int. J. Hydrogen Energy 2014, 39, 16067; b) B. Oschmann, J. Park, C. Kim, K. Char, Y.-E. Sung, R. Zentel, Chem. Mater. 2015, 27, 7011.

[52] L. Ni, Z. Wu, G. Zhao, C. Sun, C. Zhou, X. Gong, G. Diao, Small 2017, 13, 1603466.

[53] X. Liang, C. Hart, Q. Pang, A. Garsuch, T. Weiss, L. F. Nazar, Nat. Commun. 2015, 6, 5682.

[54] M.-S. Song, S.-C. Han, H.-S. Kim, J.-H. Kim, K.-T. Kim, Y.-M. Kang, H.-J. Ahn, S. X. Dou, J.-Y. Lee, J. Electrochem. Soc. 2004, 151, A791.

[55] J. Y. Hwang, H. M. Kim, S. K. Lee, J. H. Lee, A. Abouimrane, M. A. Khaleel, I. Belharouak, A. Manthiram, Y. K. Sun, Adv. Energy Mater. 2016, 6, 1501480. 
[56] S. Mei, C. J. Jafta, I. Lauermann, Q. Ran, M. Kärgell, M. Ballauff, Y. Lu, Adv. Funct. Mater. 27, 1701176.

[57] R. Tang, X. Li, Z. Ding, L. Zhang, RSC Adv. 2016, 6, 65162.

[58] Y. Hou, J. Xiao, Y. Guo, M. Qi, A. Jiang, Y. Li, J. Mater. Sci. -Mater. Electron. 2017, $28,8901$.

[59] a) S.-P. Guo, J.-C. Li, Z. Ma, Y. Chi, H.-G. Xue, J. Mater. Sci. 2017, 52, 2345; b) J. He, Q. Li, Y. Chen, C. Xu, K. Zhou, X. Wang, W. Zhang, Y. Li, Carbon 2017, 114, 111; c) P. Zhao, H. Cui, J. Luan, Z. Guo, Y. Zhou, H. Xue, Mater. Lett. 2017, 186, 62.

[60] R. Tan, J. Yang, J. Hu, K. Wang, Y. Zhao, F. Pan, Chem. Commun. 2016, 52, 986.

[61] X. Rui, H. Tan, Q. Yan, Nanoscale 2014, 6, 9889.

[62] a) X. Zhu, Z. Meng, H. Ying, X. Xu, F. Xu, W. Han, Chem. Phys. Lett. 684, 191; b) Y. Yang, F. Li, W. Li, W. Gao, H. Wen, J. Li, Y. Hu, Y. Luo, R. Li, Int. J. Hydrogen Energy 2017, 42,6665 .

[63] a) D. Yuan, G. Huang, F. Zhang, D. Yin, L. Wang, Electrochimi. Acta 2016, 203, 238; b) C. Ding, D. Su, W. Ma, Y. Zhao, D. Yan, J. Li, H. Jin, Appl. Surf. Sci. 2017, 403, 1; c) G. Kalimuldina, I. Taniguchi, J. Mater. Chem. A 2017, 5, 6937.

[64] Z. Yu, Z. Kang, Z. Hu, J. Lu, Z. Zhou, S. Jiao, Chem. Commun. 2016, 52, 10427.

[65] a) S. Dai, B. Zhao, C. Qu, D. Chen, D. Dang, B. Song, J. Fu, C. Hu, C.-P. Wong, M. Liu, Nano Energy 2017, 33, 522; b) Z. Li, X. Yu, A. Gu, H. Tang, L. Wang, Z. Lou, Nanotechnology 2017, 28, 065406.

[66] P. Long, Q. Xu, G. Peng, X. Yao, X. Xu, ChemElectroChem 2016, 3, 764.

[67] W. Zhang, X. Yan, X. Tong, J. Yang, L. Miao, Y. Sun, L. Peng, Mater. Lett. 2016, 178, 120. 
[68] a) D. Sheberla, J. C. Bachman, J. S. Elias, C.-J. Sun, Y. Shao-Horn, M. Dincă, Nat. Mater. 2017, 16, 220; b) Y. Jiao, J. Pei, D. Chen, C. Yan, Y. Hu, Q. Zhang, G. Chen, J. Mater. Chem. A 2017, 5, 1094.

[69] a) Z. Li, C. Li, X. Ge, J. Ma, Z. Zhang, Q. Li, C. Wang, L. Yin, Nano Energy 2016, 23, 15; b) X. Li, Q. Sun, J. Liu, B. Xiao, R. Li, X. Sun, J. Power Sources 2016, 302, 174.

[70] J. Zheng, J. Tian, D. Wu, M. Gu, W. Xu, C. Wang, F. Gao, M. H. Engelhard, J.-G. Zhang, J. Liu, J. Xiao, Nano Lett. 2014, 14, 2345.

[71] S. Lim, R. Lilly Thankamony, T. Yim, H. Chu, Y.-J. Kim, J. Mun, T.-H. Kim, ACS Appl. Mater. Interfaces 2015, 7, 1401.

[72] L. Bai, D. Chao, P. Xing, L. J. Tou, Z. Chen, A. Jana, Z. X. Shen, Y. Zhao, ACS Appl. Mater. Interfaces 2016, 8, 14328.

[73] Y. Mao, G. Li, Y. Guo, Z. Li, C. Liang, X. Peng, Z. Lin, Nat. Commun. 2017, 8, 14628.

[74] J. Yan, X. Liu, M. Yao, X. Wang, T. K. Wafle, B. Li, Chem. Mater. 2015, 27, 5080.

[75] J. Fanous, M. Wegner, J. Grimminger, Ä. Andresen, M. R. Buchmeiser, Chem. Mater. 2011, 23, 5024.

[76] J. Yan, X. Liu, X. Wang, B. Li, J. Mater. Chem. A 2015, 3, 10127.

[77] S. Jin, S. Xin, L. Wang, Z. Du, L. Cao, J. Chen, X. Kong, M. Gong, J. Lu, Y. Zhu, H. Ji, R. S. Ruoff, Adv. Mater. 2016, 28, 9094.

[78] Y.-J. Li, J.-M. Fan, M.-S. Zheng, Q.-F. Dong, Energy Environ. Sci. 2016, 9, 1998.

[79] J. Song, T. Xu, M. L. Gordin, P. Zhu, D. Lv, Y.-B. Jiang, Y. Chen, Y. Duan, D. Wang, Adv. Funct. Mater. 2014, 24, 1243.

[80] J. Song, M. L. Gordin, T. Xu, S. Chen, Z. Yu, H. Sohn, J. Lu, Y. Ren, Y. Duan, D. Wang, Angew. Chem. Int. Ed. 2015, 54, 4325. 
[81] G. Zhou, Y. Zhao, A. Manthiram, Adv. Energy Mater. 2015, 5, 1402263.

[82] K. Xi, S. Cao, X. Peng, C. Ducati, R. Vasant Kumar, A. K. Cheetham, Chem. Commun. 2013, 49, 2192.

[83] G. Hu, Z. Sun, C. Shi, R. Fang, J. Chen, P. Hou, C. Liu, H.-M. Cheng, F. Li, Adv. Mater. 2017, 29, 1603835.

[84] H. Kim, J. Lee, H. Ahn, O. Kim, M. J. Park, Nat. Commun. 2015, 6, 7278.

[85] G. Zheng, Q. Zhang, J. J. Cha, Y. Yang, W. Li, Z. W. Seh, Y. Cui, Nano Lett. 2013, 13, 1265.

[86] G. Zhou, L.-C. Yin, D.-W. Wang, L. Li, S. Pei, I. R. Gentle, F. Li, H.-M. Cheng, ACS Nano 2013, 7, 5367.

[87] a) W. Li, Q. Zhang, G. Zheng, Z. W. Seh, H. Yao, Y. Cui, Nano Lett. 2013, 13, 5534; b) K. Zhang, K. Xie, K. Yuan, W. Lu, S. Hu, W. Wei, M. Bai, C. Shen, J. Mater. Chem. A 2017, 5, 7309 .

[88] F. Li, Y. Su, J. Zhao, Phys. Chem. Chem. Phys. 2016, 18, 25241.

[89] Y. Qiu, W. Li, W. Zhao, G. Li, Y. Hou, M. Liu, L. Zhou, F. Ye, H. Li, Z. Wei, Nano Lett. 2014, 14, 4821.

[90] T.-Z. Hou, W.-T. Xu, X. Chen, H.-J. Peng, J.-Q. Huang, Q. Zhang, Angew. Chem. Int. Ed. 2017, 56, 8178.

[91] a) Y. Zhong, K. R. Yang, W. Liu, P. He, V. Batista, H. Wang, J. Phys. Chem. C 2017, 121, 14222; b) G. Zhou, H. Tian, Y. Jin, X. Tao, B. Liu, R. Zhang, Z. W. Seh, D. Zhuo, Y. Liu, J. Sun, J. Zhao, C. Zu, D. S. Wu, Q. Zhang, Y. Cui, PNAS 2017, 114, 840.

[92] X. Chen, H.-J. Peng, R. Zhang, T.-Z. Hou, J.-Q. Huang, B. Li, Q. Zhang, ACS Energy Letters 2017, 2, 795. 
[93] S. Zheng, P. Han, Z. Han, H. Zhang, Z. Tang, J. Yang, Sci. Rep. 2014, 4, 4842.

[94] G. Li, J. Sun, W. Hou, S. Jiang, Y. Huang, J. Geng, Nat. Commun. 2016, 7, 10601.

[95] A. Schneider, J. Janek, T. Brezesinski, Phys. Chem. Chem. Phys. 2017, 19, 8349.

[96] C. Luo, Y. Zhu, O. Borodin, T. Gao, X. Fan, Y. Xu, K. Xu, C. Wang, Adv. Funct. Mater. 2016, 26, 745 .

[97] H. J. Peng, T. Z. Hou, Q. Zhang, J. Q. Huang, X. B. Cheng, M. Q. Guo, Z. Yuan, L. Y. He, F. Wei, Adv. Mater. Interfaces 2014, 1.

[98] W. Zhou, C. Wang, Q. Zhang, H. D. Abruña, Y. He, J. Wang, S. X. Mao, X. Xiao, Adv. Energy Mater. 2015, 5.

[99] M.-q. Guo, J.-q. Huang, X.-y. Kong, H.-j. Peng, H. Shui, F.-y. Qian, L. Zhu, W.-c. Zhu, Q. Zhang, New Carbon Mater. 2016, 31, 352.

[100] X. Gu, C.-j. Tong, C. Lai, J. Qiu, X. Huang, W. Yang, B. Wen, L.-m. Liu, Y. Hou, S. Zhang, J. Mater. Chem. A 2015, 3, 16670.

[101] J. Zhang, Y. Shi, Y. Ding, L. Peng, W. Zhang, G. Yu, Adv. Energy Mater. 2017, 7, 1602876.

[102] F. Wu, J. Qian, W. Wu, Y. Ye, Z. Sun, B. Xu, X. Yang, Y. Xu, J. Zhang, R. Chen, Nano Res. 2017, 10, 426.

[103] S. Yuan, J. L. Bao, L. Wang, Y. Xia, D. G. Truhlar, Y. Wang, Adv. Energy Mater. 2016, 6,1501733 .

[104] M.-S. Kim, E. S. Shin, J.-S. Kim, W. I. Cho, S. H. Oh, J. Electroceramics 2014, 33, 142.

[105] M. Liu, Q. Li, X. Qin, G. Liang, W. Han, D. Zhou, Y.-B. He, B. Li, F. Kang, Small 2017, 13,1602539 .

[106] X. Li, K. Hu, R. Tang, K. Zhao, Y. Ding, RSC Adv. 2016, 6, 71319. 
[107] L. Jia, T. Wu, J. Lu, L. Ma, W. Zhu, X. Qiu, ACS Appl. Mater. Interfaces 2016, 8, 30248.

[108] S. Evers, T. Yim, L. F. Nazar, J. Phys. Chem. C 2012, 116, 19653.

[109] Z. Wei Seh, W. Li, J. J. Cha, G. Zheng, Y. Yang, M. T. McDowell, P.-C. Hsu, Y. Cui, Nat. Commun. 2013, 4, 1331.

[110] Q. Pang, D. Kundu, M. Cuisinier, L. F. Nazar, Nat. Commun. 2014, 5, 4759.

[111] Z. Ma, Z. Li, K. Hu, D. Liu, J. Huo, S. Wang, J. Power Sources 2016, 325, 71.

[112] Z. Yuan, H.-J. Peng, T.-Z. Hou, J.-Q. Huang, C.-M. Chen, D.-W. Wang, X.-B. Cheng, F. Wei, Q. Zhang, Nano Letters 2016, 16, 519.

[113] H. Wang, T. Zhou, D. Li, H. Gao, G. Gao, A. Du, H. Liu, Z. Guo, ACS Appl. Mater. Interfaces 2017, 9, 4320.

[114] S. S. Zhang, D. T. Tran, J. Mater. Chem. A 2016, 4, 4371.

[115] C. Zhao, C. Shen, F. Xin, Z. Sun, W. Han, Mater. Letters 2014, 137, 52.

[116] W. Tang, Z. Chen, B. Tian, H.-W. Lee, X. Zhao, X. Fan, Y. Fan, K. Leng, C. Peng, M.H. Kim, M. Li, M. Lin, J. Su, J. Chen, H. Y. Jeong, X. Yin, Q. Zhang, W. Zhou, K. P. Loh, G. W. Zheng, J. Am. Chem. Soc.2017, 139, 10133.

[117] Z. Ma, Q. Liu, S. Wang, RSC Advances 2015, 5, 2096.

[118] P. T. Dirlam, J. Park, A. G. Simmonds, K. Domanik, C. B. Arrington, J. L. Schaefer, V. P. Oleshko, T. S. Kleine, K. Char, R. S. Glass, C. L. Soles, C. Kim, N. Pinna, Y.-E. Sung, J. Pyun, ACS Appl. Mater. Interfaces 2016, 8, 13437.

[119] C. Ye, L. Zhang, C. Guo, D. Li, A. Vasileff, H. Wang, S.-Z. Qiao, Adv. Funct. Mater. 2017, 27, 1702524.

[120] Z. Liu, X. Zheng, S.-1. Luo, S.-q. Xu, N.-y. Yuan, J.-n. Ding, J. Mater. Chem. A 2016, 4, 13395. 
[121] L. Luo, S.-H. Chung, C.-H. Chang, A. Manthiram, J. Mater. Chem. A 2017, 5, 15002.

[122] I. Yoshimatsu, T. Hirai, J. i. Yamaki, J. Electrochem. Soc. 1988, 135, 2422.

[123] Y. S. Cohen, Y. Cohen, D. Aurbach, J. Phys. Chem. B 2000, 104, 12282.

[124] C. Brissot, M. Rosso, J.-N. Chazalviel, S. Lascaud, J. Power Sources 1999, 81, 925.

[125] C. Monroe, J. Newman, J. Electrochem. Soc. 2003, 150, A1377.

[126] a) Y. Lu, Z. Tu, L. A. Archer, Nat. Mater. 2014, 13, 961; b) J. Hu, G. Long, S. Liu, G. Li, X. Gao, Chem. Commun. 2014, 50, 14647.

[127] C.-P. Yang, Y.-X. Yin, S.-F. Zhang, N.-W. Li, Y.-G. Guo, Nat. Commun.2015, 6, 8058.

[128] a) N. W. Li, Y. X. Yin, C. P. Yang, Y. G. Guo, Adv. Mater.2016, 28, 1853; b) A. Basile, A. I. Bhatt, A. P. O’Mullane, Nat. Commun.2016, 7, ncomms11794.

[129] Y. Gofer, M. Ben-Zion, D. Aurbach, J. Power Sources 1992, 39, 163.

[130] K. Naoi, M. Mori, Y. Naruoka, W. M. Lamanna, R. Atanasoski, J. Electrochem. Soc.1999, 146, 462.

[131] Z. Liang, G. Zheng, C. Liu, N. Liu, W. Li, K. Yan, H. Yao, P.-C. Hsu, S. Chu, Y. Cui, Nano letters 2015, 15, 2910.

[132] X.-B. Cheng, H.-J. Peng, J.-Q. Huang, R. Zhang, C.-Z. Zhao, Q. Zhang, ACS nano 2015, $9,6373$.

[133] H. Ye, S. Xin, Y.-X. Yin, J.-Y. Li, Y.-G. Guo, L.-J. Wan, J. Am. Chem. Soc.2017, 139, 5916.

[134] a) N.-S. Choi, Y. M. Lee, J. H. Park, J.-K. Park, J. Power Sources 2003, 119, 610; b) N.S. Choi, Y. M. Lee, W. Seol, J. A. Lee, J.-K. Park, Solid State Ionics 2004, 172, 19.

[135] D. Belov, O. Yarmolenko, A. Peng, O. Efimov, Synthetic Metals 2006, 156, 745.

[136] M. Wu, Z. Wen, Y. Liu, X. Wang, L. Huang, J. Power Sources 2011, 196, 8091. 
[137] G. Zheng, S. W. Lee, Z. Liang, H.-W. Lee, K. Yan, H. Yao, H. Wang, W. Li, S. Chu, Y. Cui, Nat. Nanotechnology 2014, 9, 618.

[138] C. Yang, B. Liu, F. Jiang, Y. Zhang, H. Xie, E. Hitz, L. Hu, Nano Research 2017, 10, 4256.

[139] C. Wang, H. Xie, L. Zhang, Y. Gong, G. Pastel, J. Dai, B. Liu, E. D. Wachsman, L. Hu, Adv. Energy Mater. 2018, 8, 1701963.

[140] C.-L. Tsai, V. Roddatis, C. V. Chandran, Q. Ma, S. Uhlenbruck, M. Bram, P. Heitjans, O. Guillon, ACS Appl. Mater. Interfaces 2016, 8, 10617.

[141] W. Yang, H. Zhao, L. Chen, C. Fang, Z. Rui, L. Yang, H. Wan, J. Liu, Y. Zhou, P. Wang, Z. Zou, Chem. Eng. J. 2017, 326, 1040.

[142] K. Xi, D. He, C. Harris, Y. Wang, C. Lai, H. Li, P. R. Coxon, S. Ding, C. Wang, R. V. Kumar, Adv. Sci. 2019, 6, 1800815.

[143] C.-J. Jia, L.-D. Sun, F. Luo, X.-D. Han, L. J. Heyderman, Z.-G. Yan, C.-H. Yan, K. Zheng, Z. Zhang, M. Takano, N. Hayashi, M. Eltschka, M. Kläui, U. Rüdiger, T. Kasama, L. Cervera-Gontard, R. E. Dunin-Borkowski, G. Tzvetkov, J. Raabe, J. Am. Chem. Soc. 2008, 130, 16968.

[144] Z. Zeng, H. Zhao, P. Lv, Z. Zhang, J. Wang, Q. Xia, J. Power Sources 2015, 274, 1091.

[145] a) M. Li, X. Wu, J. Zeng, Z. Hou, S. Liao, Electrochimica Acta 2015, 182, 351; b) Y. M. Ahmed, A. Al-Mamun, A. T. Jameel, M. a. F. R. AlKhatib, M. K. Amosa, M. A. AlSaadi, J.Nanotechnology 2016, 2016.

[146] G. Partizan, B. Mansurov, B. Medyanova, A. Koshanova, M. Mansurova, B. Aliyev, X. Jiang, Eurasian Chemico-Technological J. 2016, 18, 283. 
[147] a) J. Roman, W. Neri, A. Derré, P. Poulin, Carbon 2019, 145, 556; b) J. Chinthaginjala, D. Thakur, K. Seshan, L. Lefferts, Carbon 2008, 46, 1638; c) Y. Liu, C. Pan, J. Wang, J.Mater. Sci. 2004, 39, 1091; d) L.-F. Chen, Y. Lu, L. Yu, X. W. D. Lou, Energy Environm. Sci. 2017, 10, 1777; e) L. Qie, W. Chen, X. Xiong, C. Hu, F. Zou, P. Hu, Y. Huang, Adv. Sci. 2015, 2; f) L.-S. Zhang, W. Li, Z.-M. Cui, W.-G. Song, J. Phys. Chem. C 2009, 113, 20594; g) W. Li, L.-S. Zhang, Q. Wang, Y. Yu, Z. Chen, C.-Y. Cao, W.-G. Song, J. Mater. Chem. 2012, 22, 15342; h) X. Li, W. Zhang, Y. Feng, W. Li, P. Peng, J. Yao, M. Li, C. Jiang, Electrochim. Acta 2019, 294, 173.

[148] a) Q.-T. Xu, H.-G. Xue, S.-P. Guo, Electrochim. Acta 2018, 292, 1; b) Z. Lu, N. Wang, Y. Zhang, P. Xue, M. Guo, B. Tang, Z. Bai, S. Dou, Electrochim. Acta 2018, 260, 755; c) W. Ma, X. Liu, X. Lei, Z. Yuan, Y. Ding, Chem. Eng. J. 2018, 334, 725.

[149] G. Li, X. Wang, M. H. Seo, M. Li, L. Ma, Y. Yuan, T. Wu, A. Yu, S. Wang, J. Lu, Nat. Commun.2018, 9, 705 .

[150] a) Q. Wang, W. Zhang, C. Guo, Y. Liu, C. Wang, Z. Guo, Adv. Funct. Mater. 2017, 27, 1703390; b) R. Wang, J. Li, S. Cai, Y. Zeng, H. Zhang, H. Cai, H. Tang, ChemPlusChem 2016, 81, 646; c) B. Joshi, J.-G. Lee, E. Samuel, H. S. Jo, T.-G. Kim, M. T. Swihart, W. Y. Yoon, S. S. Yoon, J. Alloys and Compounds 2017, 726, 114.

[151] Z. Li, Y. Jiang, L. Yuan, Z. Yi, C. Wu, Y. Liu, P. Strasser, Y. Huang, ACS Nano 2014, 8, 9295.

[152] a) Z. Zhang, C. Zhao, S. Min, X. Qian, Electrochim. Acta 2014, 144, 100; b) W. Zhou, J.L. Zheng, Y.-H. Yue, L. Guo, Nano Energy 2015, 11, 428; c) H.-J. Peng, Z.-W. Zhang, J.-Q. Huang, G. Zhang, J. Xie, W.-T. Xu, J.-L. Shi, X. Chen, X.-B. Cheng, Q. Zhang, Adv. Mater.2016, 28, 9551. 
[153] W. Liu, J. Jiang, K. R. Yang, Y. Mi, P. Kumaravadivel, Y. Zhong, Q. Fan, Z. Weng, Z. Wu, J. J. Cha, PNAS 2017, 114, 3578.

[154] a) J. Ye, F. He, J. Nie, Y. Cao, H. Yang, X. Ai, J. Mater. Chem. A 2015, 3, 7406; b) J. Zhang, J. Xiang, Z. Dong, Y. Liu, Y. Wu, C. Xu, G. Du, Electrochim. Acta 2014, 116, 146; c) L. Li, L. Hou, J. Cheng, T. Simmons, F. Zhang, L. T. Zhang, R. J. Linhardt, N. Koratkar, Energy Storage Mater. 2018, 15, 388.

[155] J. Ma, J. Fan, S. Chen, X. Yang, K. N. Hui, H. Zhang, C. W. Bielawski, J. Geng, ACS Appl. Mater. Interfaces 2019, 11, 13234.

[156] Z. Ma, L. Tao, D. Liu, Z. Li, Y. Zhang, Z. Liu, H. Liu, R. Chen, J. Huo, S. Wang, J. Mater. Chem. A 2017, 5, 9412.

[157] Z. Li, S. Zhang, J. Zhang, M. Xu, R. Tatara, K. Dokko, M. Watanabe, ACS Appl. Mater. Interfaces 2017, 9, 38477.

[158] H. Liu, M. Chen, P. Zeng, X. Li, J. Luo, Y. Li, T. Xing, B. Chang, X. Wang, Z. Luo, ACS Sustain. Chem. Eng. 2019.

[159] L. Zhang, Z. Chen, N. Dongfang, M. Li, C. Diao, Q. Wu, X. Chi, P. Jiang, Z. Zhao, L. Dong, Adv. Energy Mater. 2018, 8, 1802431.

[160] Q. Pang, C. Y. Kwok, D. Kundu, X. Liang, L. F. Nazar, Joule 2019, 3, 136.

[161] W. Chen, T. Lei, T. Qian, W. Lv, W. He, C. Wu, X. Liu, J. Liu, B. Chen, C. Yan, Adv. Energy Mater. 2018, 8, 1702889.

[162] J. Wang, K. Jiang, B. Shen, M. Zhen, ACS Sustain. Chem. Eng. 2019.

[163] H. Yuan, H.-J. Peng, B.-Q. Li, J. Xie, L. Kong, M. Zhao, X. Chen, J.-Q. Huang, Q. Zhang, Adv. Energy Mater. 2019, 9, 1802768.

[164] Y. Fu, J. Hu, Q. Wang, K. Li, L. Zhou, Carbon 2019, 150, 76. 
[165] L. Tan, X. Li, Z. Wang, H. Guo, J. Wang, ACS Appl. Mater. Interfaces 2018, 10, 3707.

[166] L. Kong, X. Chen, B. Q. Li, H. J. Peng, J. Q. Huang, J. Xie, Q. Zhang, Adv. Mater. 2018, 30,1705219 .

[167] G. Zhou, K. Liu, Y. Fan, M. Yuan, B. Liu, W. Liu, F. Shi, Y. Liu, W. Chen, J. Lopez, ACS Central Sci. 2018, 4, 260.

[168] G. Xu, Q.-b. Yan, P. Bai, H. Dou, P. Nie, X. Zhang, ChemistrySelect 2019, 4, 698.

[169] P. J. H. Kim, K. Kim, V. G. Pol, Carbon 2018, 131, 175.

[170] W. Cai, G. Li, K. Zhang, G. Xiao, C. Wang, K. Ye, Z. Chen, Y. Zhu, Y. Qian, Adv. Funct. Mater. 2018, 28, 1704865.

[171] S. Chen, Y. Ming, B. Tan, S. Chen, Electrochim. Acta 2020, 329, 135128.

[172] J. Zhang, Z. Li, Y. Chen, S. Gao, X. W. Lou, Angew. Chem. Int. Ed. 2018, 57, 10944.

[173] X. Song, S. Wang, G. Chen, T. Gao, Y. Bao, L.-X. Ding, H. Wang, Chem. Eng. J. 2018, $333,564$.

[174] L. Li, L. Chen, S. Mukherjee, J. Gao, H. Sun, Z. Liu, X. Ma, T. Gupta, C. V. Singh, W. Ren, Adv. Mater. 2017, 29, 1602734.

[175] a) J. Xu, D. Su, W. Zhang, W. Bao, G. Wang, J. Mater. Chem. A 2016, 4, 17381; b) C. Zhang, Y. Lin, J. Liu, J. Mater. Chem. A 2015, 3, 10760.

[176] M. Fantauzzi, B. Elsener, D. Atzei, A. Rigoldi, A. Rossi, RSC Adv. 2015, 5, 75953.

[177] a) M. Yu, Z. Wang, Y. Wang, Y. Dong, J. Qiu, Adv. Energy Mater. 2017, 7, 1700018; b) K. Park, J. H. Cho, J.-H. Jang, B.-C. Yu, A. T. De La Hoz, K. M. Miller, C. J. Ellison, J. B. Goodenough, Energy Environ. Sci. 2015, 8, 2389; c) Y. Jiang, F. Chen, Y. Gao, Y. Wang, S. Wang, Q. Gao, Z. Jiao, B. Zhao, Z. Chen, J. Power Sources 2017, 342, 929.

[178] B.-C. Yu, J.-W. Jung, K. Park, J. B. Goodenough, Energy Environ. Sci.2017, 10, 86. 
[179] S. Biniak, G. Szymański, J. Siedlewski, A. Świątkowski, Carbon 1997, 35, 1799.

[180] a) X.-g. Yu, J.-y. Xie, J. Yang, H.-j. Huang, K. Wang, Z.-s. Wen, J. Electroanalytical Chem. 2004, 573, 121; b) B. Meyer, Chem. Rev.1976, 76, 367.

[181] X. Wang, J. Hu, Y. Su, J. Hao, F. Liu, S. Han, J. An, J. Lian, Chem. - A European J.2017, 23, 4128.

[182] J. Kapitan, L. Hecht, P. Bouř, Phys. Chem. Chem. Phys. 2008, 10, 1003.

[183] C. Sandroff, D. Herschbach, J. Phys. Chem. 1982, 86, 3277.

[184] a) Z. Cheng, H. Abernathy, M. Liu, J. Phys. Chem. C 2007, 111, 17997; b) X. Song, X. Li, Z. Bai, B. Yan, D. Xiong, L. Lin, H. Zhao, D. Li, Y. Shao, Carbon 2018, 133, 14.

[185] N. A. Jarrah, F. Li, J. G. van Ommen, L. Lefferts, J. Mater. Chem. 2005, 15, 1946.

[186] a) H. Wei, E. F. Rodriguez, A. S. Best, A. F. Hollenkamp, D. Chen, R. A. Caruso, Adv. Energy Mater. 2017, 7, 1601616; b) S. Zeng, L. Li, D. Zhao, J. Liu, W. Niu, N. Wang, S. Chen, J. Phys. Chem. C 2017, 121, 2495.

[187] X. Song, X. Li, Z. Bai, B. Yan, D. Li, X. Sun, Nano Energy 2016, 26, 533.

[188] a) Y. Li, K. K. Fu, C. Chen, W. Luo, T. Gao, S. Xu, J. Dai, G. Pastel, Y. Wang, B. Liu, ACS nano 2017, 11, 4801; b) G. Li, W. Lei, D. Luo, Y. P. Deng, D. Wang, Z. Chen, Adv. Energy Mater. 2018, 8, 1702381; c) S. Huang, L. Zhang, J. Wang, J. Zhu, P. K. Shen, Nano Research 2018, 11, 1731.

[189] a) Y. Wang, R. Zhang, Y.-c. Pang, X. Chen, J. Lang, J. Xu, C. Xiao, H. Li, K. Xi, S. Ding, Energy Storage Mater. 2019, 16, 228; b) J. He, G. Hartmann, M. Lee, G. S. Hwang, Y. Chen, A. Manthiram, Energy Environ. Sci. 2019, 12, 344. 
[190] a) Y. You, Y. Ye, M. Wei, W. Sun, Q. Tang, J. Zhang, X. Chen, H. Li, J. Xu, Chem. Eng. J. 2019, 355, 671; b) Y. Zhong, D. Chao, S. Deng, J. Zhan, R. Fang, Y. Xia, Y. Wang, X. Wang, X. Xia, J. Tu, Adv. Funct. Mater. 2018, 28, 1706391.

[191] C. Li, J. Shi, L. Zhu, Y. Zhao, J. Lu, L. Xu, Nano Research 2018, 11, 4302.

[192] D. Lv, J. Zheng, Q. Li, X. Xie, S. Ferrara, Z. Nie, L. B. Mehdi, N. D. Browning, J. G. Zhang, G. L. Graff, Adv. Energy Mater. 2015, 5, 1402290.

[193] Q. Zhao, Q. Zhu, J. Miao, Z. Guan, H. Liu, R. Chen, Y. An, F. Wu, B. Xu, ACS Appl. Mater. Interfaces 2018, 10, 10882.

[194] Q. Pang, D. Kundu, L. F. Nazar, Mater. Horizons 2016, 3, 130.

[195] Y. Zhong, X. Xia, S. Deng, J. Zhan, R. Fang, Y. Xia, X. Wang, Q. Zhang, J. Tu, Adv. Energy Mater. 2018, 8, 1701110.

[196] L. Kong, B. Q. Li, H. J. Peng, R. Zhang, J. Xie, J. Q. Huang, Q. Zhang, Adv. Energy Mater. 2018, 8, 1800849.

[197] X. Liang, Y. Rangom, C. Y. Kwok, Q. Pang, L. F. Nazar, Adv. Mater. 2017, 29, 1603040.

[198] J. Park, B.-C. Yu, J. S. Park, J. W. Choi, C. Kim, Y.-E. Sung, J. B. Goodenough, Adv. Energy Mater. 2017, 7, 1602567.

[199] a) Z. Xiao, L. Li, Y. Tang, Z. Cheng, H. Pan, D. Tian, R. Wang, Energy Storage Mater. 2018, 12, 252; b) P. Chiochan, S. Kaewruang, N. Phattharasupakun, J. Wutthiprom, T. Maihom, J. Limtrakul, S. Nagarkar, S. Horike, M. Sawangphruk, Sci. Rep. 2017, 7, 17703; c) G. Zhou, E. Paek, G. S. Hwang, A. Manthiram, Nat. Commun. 2015, 6, 7760.

[200] P. Metcalf, P. Fanwick, Z. Kakol, J. Honig, J. Solid State Chem.1993, 104, 81.

[201] D. Bérardan, S. Franger, A. K. Meena, N. Dragoe, J. Mater. Chem. A 2016, 4, 9536. 
[202] K. Yan, Z. Lu, H.-W. Lee, F. Xiong, P.-C. Hsu, Y. Li, J. Zhao, S. Chu, Y. Cui, Nat. Energy 2016, 1, 1.

[203] Z. Lu, Q. Liang, B. Wang, Y. Tao, Y. Zhao, W. Lv, D. Liu, C. Zhang, Z. Weng, J. Liang, Adv. Energy Mater. 2019, 9, 1803186.

[204] S. Wu, Z. Zhang, M. Lan, S. Yang, J. Cheng, J. Cai, J. Shen, Y. Zhu, K. Zhang, W. Zhang, Adv. Mater. 2018, 30, 1705830.

[205] A. Wang, S. Xie, R. Zhang, Y. She, C. Chen, M. K. Leung, C. Niu, H. Wang, Nanoscale $A d v .2019,1,656$.

[206] S.-S. Chi, Y. Liu, N. Zhao, X. Guo, C.-W. Nan, L.-Z. Fan, Energy Storage Mater. 2019, $17,309$.

[207] S. Li, Q. Liu, J. Zhou, T. Pan, L. Gao, W. Zhang, L. Fan, Y. Lu, Adv. Funct. Mater. 2019, $29,1808847$.

[208] S. S. Chi, Y. Liu, W. L. Song, L. Z. Fan, Q. Zhang, Adv. Funct. Mater. 2017, 27, 1700348.

[209] X. Shen, Y. Li, T. Qian, J. Liu, J. Zhou, C. Yan, J. B. Goodenough, Nat. Commun. 2019, $10,1$.

[210] N. Li, W. Wei, K. Xie, J. Tan, L. Zhang, X. Luo, K. Yuan, Q. Song, H. Li, C. Shen, Nano lett. 2018, 18, 2067.

[211] R. Zhang, X. B. Cheng, C. Z. Zhao, H. J. Peng, J. L. Shi, J. Q. Huang, J. Wang, F. Wei, Q. Zhang, Adv. Mater. 2016, 28, 2155.

[212] Y. Zhang, Y. Shi, X. C. Hu, W. P. Wang, R. Wen, S. Xin, Y. G. Guo, Adv. Energy Mater. 2020, 10, 1903325. 
[213] H. Zhao, D. Lei, Y. B. He, Y. Yuan, Q. Yun, B. Ni, W. Lv, B. Li, Q. H. Yang, F. Kang, Adv. Energy Mater. 2018, 8, 1800266.

[214] H. Chen, A. Pei, D. Lin, J. Xie, A. Yang, J. Xu, K. Lin, J. Wang, H. Wang, F. Shi, Adv. Energy Mater. 2019, 9, 1900858.

[215] Y. Yuan, F. Wu, Y. Bai, Y. Li, G. Chen, Z. Wang, C. Wu, Energy Storage Mater. 2019, $16,411$.

[216] G. Li, Z. Liu, D. Wang, X. He, S. Liu, Y. Gao, A. AlZahrani, S. H. Kim, L. Q. Chen, D. Wang, Adv. Energy Mater. 2019, 9, 1900704.

[217] Y. Feng, C. Zhang, X. Jiao, Z. Zhou, J. Song, Energy Storage Mater. 2020, 25, 172.

[218] Y. Nan, S. Li, B. Li, S. Yang, Nanoscale 2019, 11, 2194.

[219] K. Chen, R. Pathak, A. Gurung, E. A. Adhamash, B. Bahrami, Q. He, H. Qiao, A. L. Smirnova, J. J. Wu, Q. Qiao, Energy Storage Mater. 2019, 18, 389.

[220] Z. Cao, B. Li, S. Yang, Adv. Mater. 2019, 31, 1901310.

[221] Z. Zeng, W. Li, Q. Wang, X. Liu, Adv. Sci. 2019, 6, 1900711.

[222] R. Yan, M. Oschatz, F. Wu, Carbon 2020, 161, 162.

[223] H. Li, J. Wang, Y. Zhang, Y. Wang, A. Mentbayeva, Z. Bakenov, J. Power Sources 2019, 437, 226901.

[224] J. Zhou, S. Li, X. Ji, W. Sun, Y. Yang, Chem. Eng. J. 2020, 382, 122843.

[225] C. Wang, Y. Yi, H. Li, P. Wu, M. Li, W. Jiang, Z. Chen, H. Li, W. Zhu, S. Dai, Nano Energy 2020, 67, 104253.

[226] V. Bharti, A. Gangadharan, T. N. Rao, C. S. Sharma, Mater. Today Commun. 2020, 22, 100717. 
[227] L. L. Kong, L. Wang, Z. C. Ni, S. Liu, G. R. Li, X. P. Gao, Adv. Funct. Mater. 2019, 29, 1808756.

[228] Z. Y. Wang, L. Wang, S. Liu, G. R. Li, X. P. Gao, Adv. Funct. Mater. 2019, 29, 1901051.

[229] Y. Li, S. Lin, D. Wang, T. Gao, J. Song, P. Zhou, Z. Xu, Z. Yang, N. Xiao, S. Guo, Adv. Mater.2020, 32, 1906722.

[230] Y. T. Liu, D. D. Han, L. Wang, G. R. Li, S. Liu, X. P. Gao, Adv. Energy Mater. 2019, 9, 1803477.

[231] B. Zhang, C. Luo, Y. Deng, Z. Huang, G. Zhou, W. Lv, Y. B. He, Y. Wan, F. Kang, Q. H. Yang, Adv. Energy Mater. 2020, 10, 2000091.

[232] Y. Boyjoo, H. Shi, E. Olsson, Q. Cai, Z. S. Wu, J. Liu, G. Q. Lu, Adv. Energy Mater. 2020, 2000651.

[233] Z. Yu, M. Liu, D. Guo, J. Wang, X. Chen, J. Li, H. Jin, Z. Yang, X. a. Chen, S. Wang, Angew. Chem. Int. Ed. 2020, 59, 6406.

[234] J. L. Cheong, A. A. AbdelHamid, J. Y. Ying, Nano Energy 2019, 66, 104114.

[235] B. Tang, H. Wu, X. Du, X. Cheng, X. Liu, Z. Yu, J. Yang, M. Zhang, J. Zhang, G. Cui, Small 2020, 1905737.

[236] J. Wu, S. Ding, S. Ye, C. Lai, J.Energy Chem.2020, 42, 27.

[237] J. S. Yeon, S. H. Park, J. Suk, H. Lee, H. S. Park, Chem. Eng. J. 2020, 382, 122946.

[238] G. Chen, Y. Li, W. Zhong, F. Zheng, J. Hu, X. Ji, W. Liu, C. Yang, Z. Lin, M. Liu, Energy Storage Mater. 2020, 25, 547.

[239] L. Fan, H. Wu, X. Wu, M. Wang, J. Cheng, N. Zhang, Y. Feng, K. Sun, Electrochim. Acta 2019, 295, 444 . 
[240] Z.-Y. Wang, D.-D. Han, S. Liu, G.-R. Li, T.-Y. Yan, X.-P. Gao, Electrochim. Acta 2020, $337,135772$.

[241] W. Ge, L. Wang, C. Li, C. Wang, D. Wang, Y. Qian, L. Xu, J. Mater. Chem. A 2020, 8, 6276. 